\title{
Sliding Mode Control for Singularly Perturbed Systems
}

by

\author{
Ayman EM Ahmed
}

\author{
A thesis submitted to \\ The Faculty of Graduate Studies and Research \\ In partial fulfillment of \\ The requirement for the degree of
}

Doctor of Philosophy

Ottawa Carleton Institute for Electrical and Computer Engineering

Faculty of Engineering

Department of Systems and Computer Engineering

Carleton University 


$\begin{array}{ll}\begin{array}{l}\text { Library and } \\ \text { Archives Canada }\end{array} & \begin{array}{l}\text { Bibliothèque et } \\ \text { Archives Canada }\end{array} \\ \begin{array}{l}\text { Published Heritage } \\ \text { Branch }\end{array} & \begin{array}{l}\text { Direction du } \\ \text { Patrimoine de l'édition }\end{array} \\ \begin{array}{l}\text { 395 Wellington Street } \\ \text { Ottawa ON K1A ON4 }\end{array} & \begin{array}{l}\text { 395, rue Wellington } \\ \text { Ottawa ON K1A ON4 } \\ \text { Canada }\end{array} \\ \end{array}$

Your file Votre référence ISBN: 0-494-00794-X

Our file Notre référence

ISBN: 0-494-00794-X

NOTICE:

The author has granted a nonexclusive license allowing Library and Archives Canada to reproduce, publish, archive, preserve, conserve, communicate to the public by telecommunication or on the Internet, loan, distribute and sell theses worldwide, for commercial or noncommercial purposes, in microform, paper, electronic and/or any other formats.

The author retains copyright ownership and moral rights in this thesis. Neither the thesis nor substantial extracts from it may be printed or otherwise reproduced without the author's permission.
AVIS:

L'auteur a accordé une licence non exclusive permettant à la Bibliothèque et Archives Canada de reproduire, publier, archiver, sauvegarder, conserver, transmettre au public par télécommunication ou par l'Internet, prêter, distribuer et vendre des thèses partout dans le monde, à des fins commerciales ou autres, sur support microforme, papier, électronique et/ou autres formats.

L'auteur conserve la propriété du droit d'auteur et des droits moraux qui protège cette thèse. $\mathrm{Ni}$ la thèse ni des extraits substantiels de celle-ci ne doivent être imprimés ou autrement reproduits sans son autorisation.
In compliance with the Canadian

Privacy Act some supporting forms may have been removed from this thesis.

While these forms may be included in the document page count, their removal does not represent any loss of content from the thesis.
Conformément à la loi canadienne sur la protection de la vie privée, quelques formulaires secondaires ont été enlevés de cette thèse.

Bien que ces formulaires aient inclus dans la pagination, il n'y aura aucun contenu manquant.

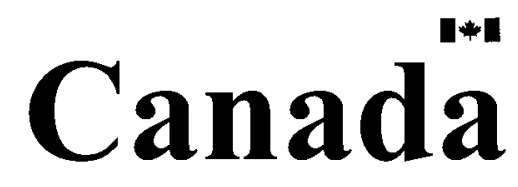




\section{ABSTRACT}

The design of sliding mode control for singularly perturbed systems is investigated in this thesis. A number of methods of sliding mode control for singularly perturbed system are presented. A single sliding mode controller has been proposed to control the full order singularly perturbed system. The design method used for this controller is based on the reduced mathematical model of both the slow and the fast subsystems. The design objective is to achieve the desired performance with feedback based only on the slow states.

An accurate decoupling method, known as the diagonalization method, is used to obtain the reduced order fast and slow subsystems. We design the single sliding mode controller based on the slow subsystem. We discuss and prove convergence and stability for the proposed controller, and present some numerical results. A maximum limit for the perturbation parameter $\mathcal{E}$ that guarantee the stability of the proposed controller is shown. The sliding mode $H^{\infty}$ frequency shaped control method is used for the proposed controller to design a frequency shaped sliding surface. By using this technique we can suppress the high frequency modes of the fast subsystem. The fuzzy sliding mode control algorithm is applied to the proposed controller. The fuzzy logic acts as a supervisory system to connect two different sliding mode controllers together, which allows us to obtain a nonlinear sliding surface.

Examples that demonstrate the usefulness of these procedures are presented. The results indicate that the proposed controller has similar or better performance compared to other control strategies. 


\section{ACKNOWLEDGEMENT}

I am sincerely grateful to my supervisor Professor Howard Schwartz for his immense help, excellent guidance and encouragement throughout my research. He has helped me to learn and understand many facts. I owe him many thanks for his support.

I would also like to express my deepest gratitude to Professor Victor Aitken for his guidance and helpful reviews to improve this thesis.

I have been supported, encouraged and loved by my wife and my three children. I would like to express my sincerest thanks for their support and hopefully I can have enough time in life to pay them back.

Foremost, I extend my deepest thanks to the department staff Darlene, Blazenka, Jennifer and Christine for their help. I enjoyed also the friendship I made with A. Mokhtar, Ehab, David and John.

Finally, I would like to thank the Egyptian government for their financial support. 


\section{Table of Contents}

\section{Introduction}

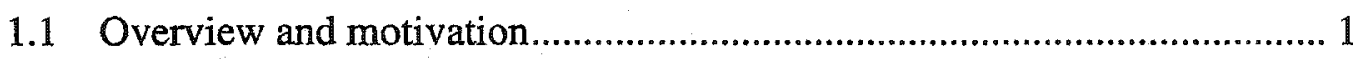

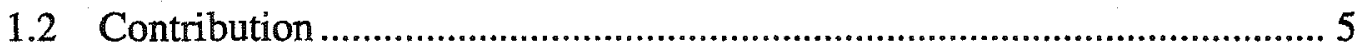

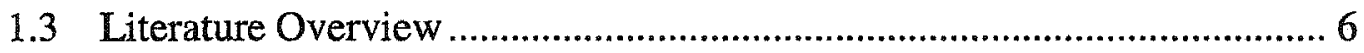

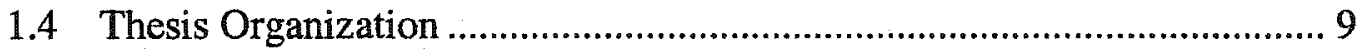

\section{Review of Siding Mode Control}

2.1 Introduction

2.2 Equivalent Control Method........................................................................14

2.2.1 Sliding surface design ....................................................................14

2.2.2 Controller design ............................................................................

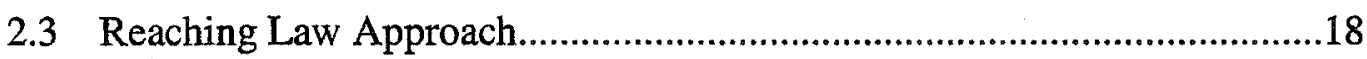

2.4 Robustness and Invariance......................................................................19

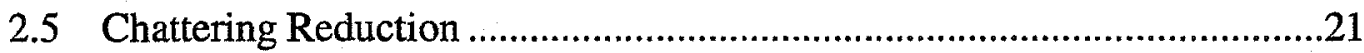

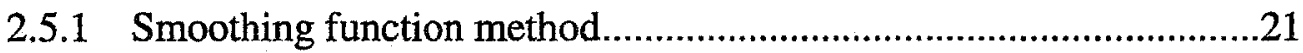

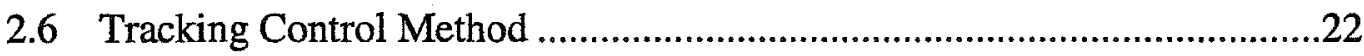

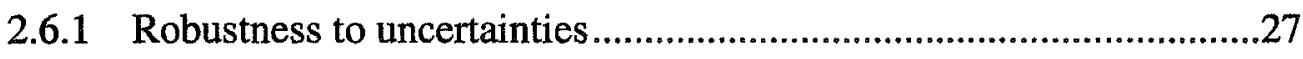

2.7 Chapter summary 30

\section{Singular Perturbation Method}

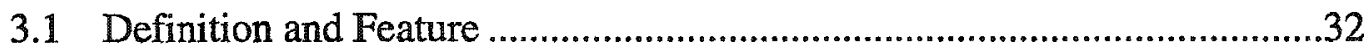

3.2 Quasi Steady State Method .........................................................................33

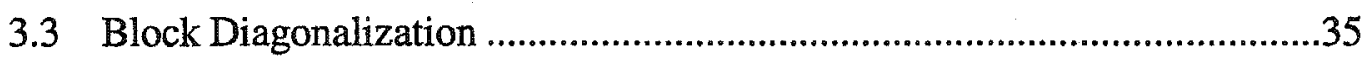

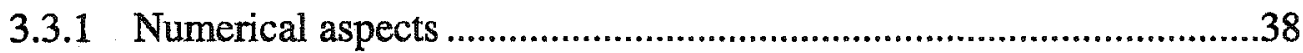

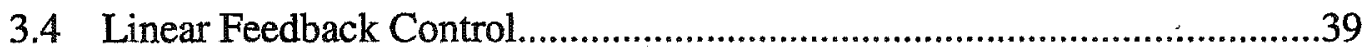

3.4.1 Introduction...................................................................................

3.4.2 Composite state feedback control ......................................................39

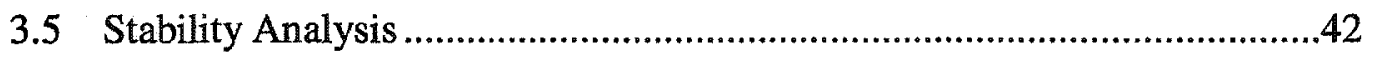




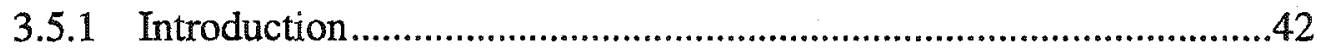

3.5.2 Lyapunov stability analysis for singular perturbed system.................43

3.5 Example of Flexible Shaft ......................................................................47

4 Review of Sliding Mode Control Methods for Singularly Perturbed Systems

4.1 Model Reduction Using a Singularly Perturbed System …..........................53

4.2 Slow Sliding Mode Control Design ...........................................................54

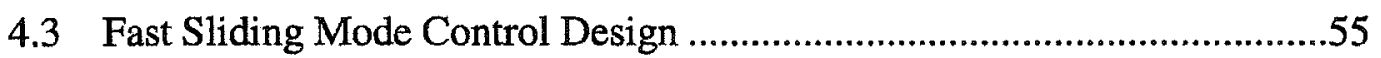

4.4 Composite Controller.............................................................................58

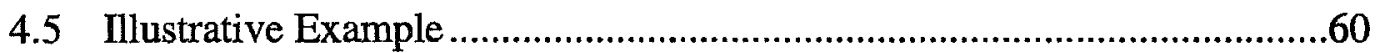

\section{New Sliding Mode Controllers for Singularly Perturbed}

\section{Systems}

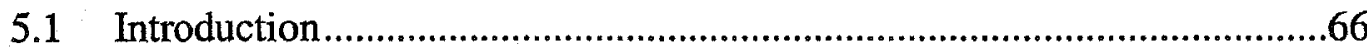

5.2 Accurate Reduced Order Singular Perturbed Model for Sliding Mode Controller....................................................................68

5.2.1 Sliding mode control design using diagonalization method..............69

5.2.2 Slow sliding mode control design .....................................................70

5.2.3 Fast sliding mode controller design ....................................................71

5.2.4 Slow sliding mode controller for the full order model .......................72

5.3 Illustrative Examples ................................................................................72

5.4 Stability Analysis ....................................................................................8

5.4.1 Stability analysis for the full order model with single sliding mode controller.......................................................8

5.4.2 Illustrative example ..............................................................................95

6 Sliding Surface Design in The Frequency Domain

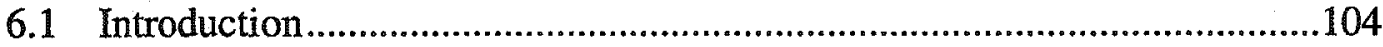

6.2 Sliding Mode using LQ Approach ...............................................................107

6.3 Frequency Shaped LQ Approach .................................................................109 
6.4 Review of the $H^{\infty}$ Approach ................................................................111

6.4.1 $H^{\text {o }}$ Optimal control design ............................................................115

6.5 Frequency Shaped Sliding Mode Control

for Singular Perturbed Systems ...............................................................117

\section{Fuzzy Sliding Mode Control For a Singularly Perturbed System}

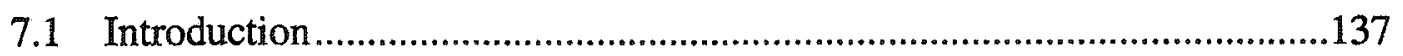

7.2 Sliding Mode Fuzzy Logic Control (SMFLC).........................................140

7.3 Fuzzy sliding Mode Control for a Singularly Perturbed Systems ...............141

7.3.1 Fuzzy sliding mode controller ..........................................................142

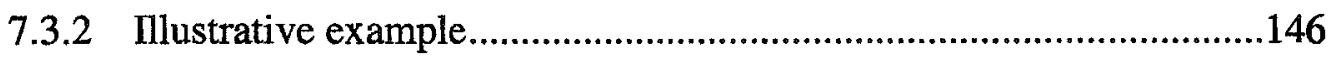

8 Conclusions and Future Work

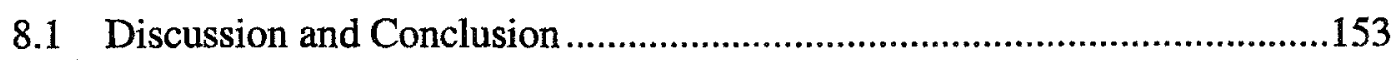

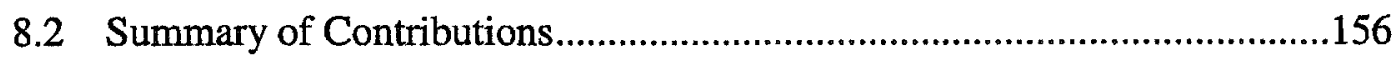

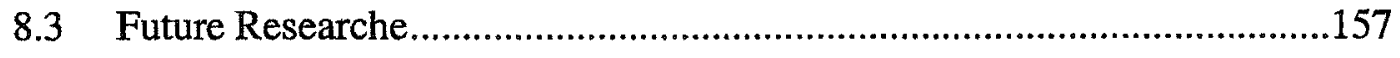

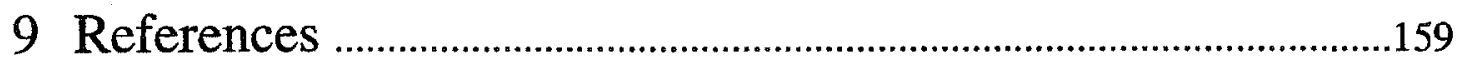

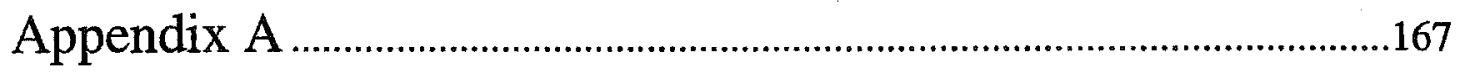




\section{List of Figures}

Figure 2.1: Block diagram of switching feedback ........................................11

Figure 2.2: Phase plane plots for the switched feedback plant .............................13

Figure 2.3: The construction of the boundary layer in the case that $n=2 \ldots \ldots \ldots \ldots \ldots . . .26$

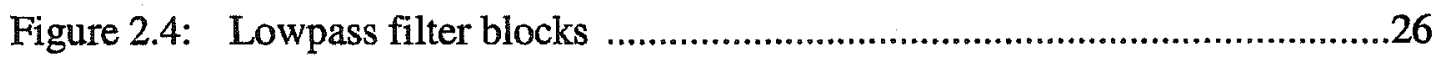

Figure 2.5: Dynamic structure of the controlled system .....................................29

Figure 3.1: Simplified flexible robot .........................................................48

Figure 3.2.a: Closed loop time response of manipulator angular velocity..............51

Figure 3.2.b: Closed loop time response of rotor angular velocity ........................51

Figure 4.1.a: Full order closed loop time response (slow subsystem) .................63

Figure 4.1.b: Full order closed loop time response (Fast subsystem) ..................64

Figure 4.2.a: Full order closed loop time response with chattering reduction in the slow subsystem ..............................................64

Figure 4.2.b: Full order closed loop time response with chattering reduction in the slow subsystem ..............................................65

Figure 5.1.a: Full order closed loop time response for two sliding mode

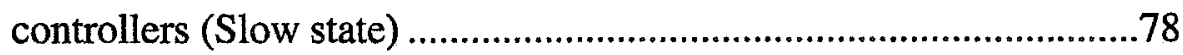

Figure 5.1.b: Full order closed loop time response for two sliding mode

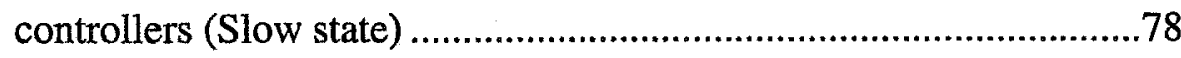

Figure 5.1.c: Full order closed loop time response for two sliding mode

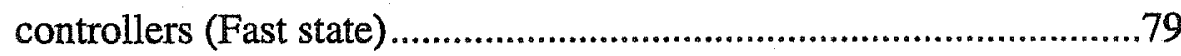

Figure 5.1.d: Full order closed loop time response for two sliding mode controllers (Fast state) ..............................................................79

Figure 5.2.a: Full order closed loop time response for single sliding mode controller (Slow state) using diagonalization method

Figure 5.2.b: Full order closed loop time response for single sliding mode controller (Slow state) using quasi steady state method .80

Figure 5.3.a: Full order closed loop time response for a single sliding mode controller (Slow state) 
Figure 5.3.b: Full order closed loop time response for a single sliding mode controller (fast state)

Figure 5.4: Phase plane for the single sliding mode controller with boundary layer .82

Figure 5.5.a: Closed loop time response for the control output (Composite and single controllers)

Figure 5.5.b: Closed loop time response for the control output

(Composite and single controllers)

Figure 5.6.a: Full order closed loop time response for a single sliding mode controller (Slow State)

Figure 5.6.b: Full order closed loop time response for a single sliding mode controller (Fast state)

Figure 5.7.a: Full order closed loop time response for Single Sliding Mode controller with a boundary Layer with a different slope (Slow State) .86

Figure 5.7.b: Full order closed loop time response for a single sliding mode controller with a boundary Layer with a different slope (Fast State). .86

Figure 5.8.a: Full order closed loop time response for sliding mode control $\varepsilon=0.005$ (Slow state) .98

Figure 5.8.b: Full order closed loop time response for sliding mode control

$$
\varepsilon=0.005 \text { (Fast state) }
$$

Figure 5.9.a: Full order closed loop time response for sliding mode control

$$
\varepsilon=0.05 \text { (Slow state) }
$$

Figure 5.9.b: Full order closed loop time response for sliding mode control

$$
\varepsilon=0.05 \text { (fast state) }
$$

Figure 5.10.a: Full order closed loop time response for sliding mode control

$$
\varepsilon=0.3 \text { ( slow state) }
$$

Figure 5.10.b: Full order closed loop time response for sliding mode control

$$
\varepsilon=0.3 \text { (fast state) }
$$

Figure 5.11.a: Full order closed loop time response for sliding mode control $\varepsilon=0.5$ (slow state) 
Figure 5.11.b: Full order closed loop time response for sliding mode control

$$
\varepsilon=0.5 \text { (fast state) }
$$

Figure 5.12: The maximum perturbation parameter versus the slope of sliding surface.

Figure 6.1: Standard weighted closed loop system ........................................112

Figure 6.2: $\quad$ Linear Fraction Transformation (LFT) .....................................113

Figure 6.3: Custom weighted closed loop system ...........................................118

Figure 6.4: $\quad H^{\infty}$ frequency shaped sliding mode control .................................122

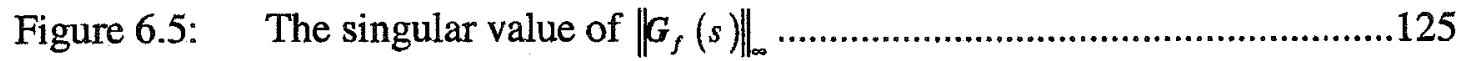

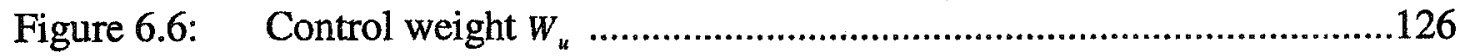

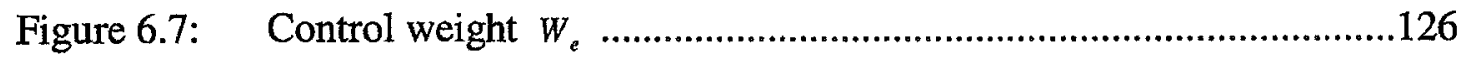

Figure 6.8: Weighted closed loop slow subsystem .......................................128

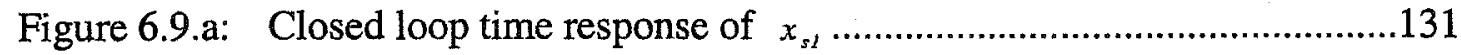

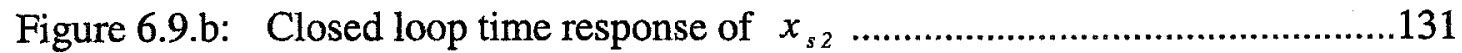

Figure 6.10.a: Closed loop time response of $x$...............................................132

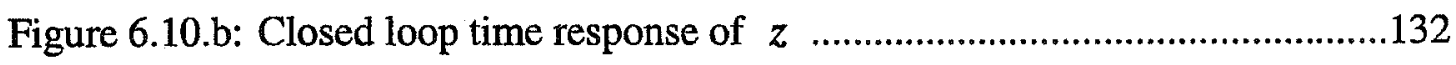

Figure 6.11: Closed loop time response of slow state for different sliding surface.......................................................133

Figure 6.12: Closed loop time response of slow state ......................................133

Figure 6.13: Closed loop time response of slow state for different sliding surface.......................................................134

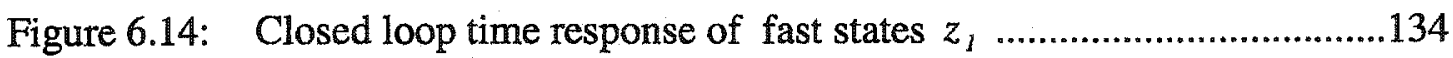

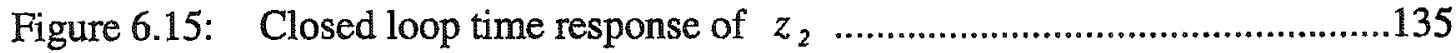

Figure 6.16: Phase plane plot for frequency shaped sliding mode controller ........135

Figure 6.17: Phase plane plot for sliding mode controller ..................................136

Figure 7.1: Classical and Fuzzy sets..............................................................138

Figure 7.2: Basic configuration of fuzzy logic controller .................................139

Figure 7.2: Time optimal control switching surface .......................................143

Figure 7.4: Fuzzy Sliding Mode Controller .................................................145

Figure 7.5: Supervisory fuzzy logic controller...............................................145 


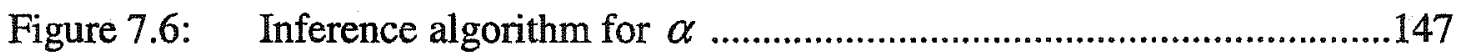

Figure 7.7: Closed loop time response of $\boldsymbol{x}$...................................................149

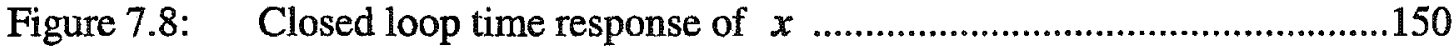

Figure 7.9: Closed loop time response of $\boldsymbol{x}$ (Slotine approach) ........................150

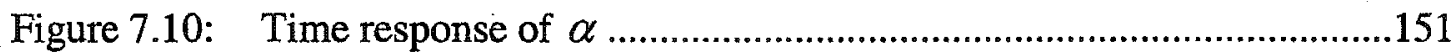

Figure 7.11.a: Closed loop time response of $\boldsymbol{x}$ (slow states) ...............................151

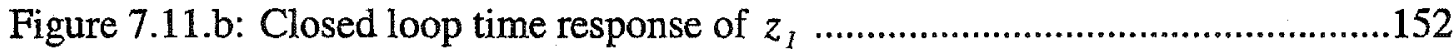

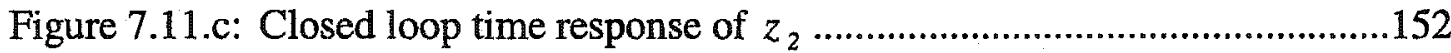




\section{Chapter 1 Introduction}

\subsection{Overview and motivation}

Sliding mode control was originally proposed by Utkin [56] in the late 1970's. Since then this control methodology has received substantial attention in the research community. The key advantage to the sliding mode technique is its robustness to a certain class of model uncertainty.

However some systems that are controlled by sliding mode controllers exhibit a disturbing chattering phenomenon. A sliding mode controller is essentially a high gain switching controller. The idea is to keep the trajectory of the system on a particular surface in the phase space. In a two dimensional system this would reduced to following a line in the phase plane. If the trajectory moved off the sliding surface a large control signal would be commanded. For example if the trajectory moved below the sliding surface a large positive signal would be commanded and if the trajectory moved above the sliding surface a large negative command signal would be commanded. If this switching could be done infinitely fast then the system trajectory will move smoothly along the specified sliding surface in the phase plane.

Of course, in practice, one can never switch anything infinitely fast. Therein lies the problem in implementing the sliding mode controller. The result is that the system under sliding mode control produces a high frequency control signal. This often causes the system to shake or "chatter". The explanation given by researchers in this field, for this 
chattering phenomena, is that it is caused by "unmodeled high frequency dynamics" which to a large degree is nothing more than a truism.

It is impossible to mathematically model a physical dynamic system perfectly. As such, one can always argue that there are "high frequency unmodeled dynamics". In many practical applications, one component of these "high frequency unmodeled dynamics" is the lightly damped structural mode of the system. For example, the lightly damped high frequency structure modes could be due to flexibility and elasticity in steel shaft, support structures gears and pulleys. High frequency electrical modes could be due to motor and line inductance and capacitance.

To address the chattering problem researchers in the field, notably Slotine [47][48][49], proposed to design a "boundary layer" around the sliding surface. The proposed "boundary layer" is a method of filtering the switched high magnitude control signal. The design procedure is largely iterative. The idea is to choose a boundary layer "wide enough" such that unmodeled high frequency dynamics are not excited. The wider the boundary layer around the sliding surface the lower will be the bandwidth of the filter acting on the switching control. In practice, when the system trajectory is inside the boundary layer, the controller is not switching but instead reverts to a PD controller. The disadvantage to this is that the robustness to disturbance and model uncertainty which is the justification for using sliding mode control is lost. Slotine [48], derives the guaranteed error bounds for a trajectory tracking controller and relates these error bounds to the width of the boundary layer. Therefore the design procedure is to iteratively widen the boundary layer until the chattering or shaking of the system is no longer a problem and then calculate the guaranteed error bounds. 
During the past two decades numerous applications of sliding mode control have been presented [10][23]. However, little if any, further research has been done on the interaction of sliding mode control with the high frequency dynamics. The goal of this research is to further investigate the interaction between the high frequency dynamic modes of the plant and the sliding mode controller and to improve the design of sliding mode controllers for such systems.

In parallel with the research into sliding mode control, other researchers were investigating two time scale systems and singularly perturbed subsystems. These are dynamic systems that are composed of two sets of dynamic modes or eigenvalues. One set of dynamic modes has relatively slow time constants and the other dynamic modes have relatively fast time constants. The dynamic modes associated with the slow time constants are referred to as the slow subsystem and the dynamic modes associated with the fast time constants are referred to as the fast subsystem.

Modeling a system in singularly perturbed form allows one to specify uncertainty in the high frequency dynamics in terms of a single parameter referred to as $\varepsilon$, in the traditional literature. Furthermore, there is a substantial quantity of literature analyzing the stability and control of systems in singularly perturbed form [5][7][18][25][30]. We could then rely on some of these theoretical results in our own work.

Therefore, to study the effects of sliding mode control on high frequency dynamics we chose to model our system in singularly perturbed form. The high frequency dynamics are explicitly defined by the model. The literature published on sliding mode control for singularly perturbed system is very scarce. There are only a few articles on the use of sliding mode control for singularly perturbed systems $[1][11][22][25][26][50][60]$. 
Furthermore, in all the articles published the authors are not interested in investigating the effect of sliding mode control on the high frequency dynamics. They are interested in designing two separate sliding mode controllers, one for the slow subsystem and a separate sliding mode controller for the fast subsystem. This seems somewhat strange since access to the high frequency dynamics states is usually not available. For example one typically will not have a strain gauge on a flexible shaft. They also decouple the singularly perturbed system into slow and fast subsystems by using quasi steady state methods. This brings in a new source of error.

In contrast to the available literature we are proposing to design and analyze the effect of a single sliding mode controller for a singularly perturbed system. The single sliding mode controller is designed based on a quasi steady state decoupling algorithm which separates the system into high and low frequency dynamics. The resulting zeroth order subsystem, which can be thought of as the slow subsystem, is controlled by the single sliding mode controller. We then examine the behavior of the full order system, which includes the fast dynamics under the control of the single sliding mode controller. This research provides important insights into how the sliding mode control interacts with the high frequency dynamics.

An advantage of using the proposed controller is that the control law in this case will depend only on the slow state. As a result there is no need for measurement of the fast state, which is usually difficult to measure. 


\subsection{Contribution}

The idea of using a singularly perturbed system to examine and investigate the effect of sliding mode control on high frequency dynamics is an original contribution of this work. Given that we are using this original approach to investigate the effects of sliding mode control on the high frequency dynamics we have made a number of important contributions.

The research began with implementing the sliding mode controller on the singularly perturbed system and demonstrating the chattering phenomena. This is shown in Figure 4.1. Then using the boundary layer approach we demonstrate that the chattering can be suppressed just as one would obtain from the literature. This is shown in Figure 4.2.

In chapter 5 , we replicate the result in the literature by implementing two sliding mode controllers. We use the quasi steady state decoupling approach to define the slow and fast subsystems. The example is illustrated in Figure 5.1. This example is primarily used to verify our simulation and control techniques in comparison to the available literature [22]. We then use a diagonalization method to more accurately decouple the singularly perturbed system. Using the diagonalization approach one does not introduce the approximation error associated with the quasi steady state methods. We can then demonstrate, as in Figure 5.1 and 5.3 that the diagonalization method results in improved performance when using either a single sliding mode controller or two sliding mode controllers. The disadvantage of the diagonalization method is that one must measure states that are typically inaccessible. Although the results of using the diagonalization method point to an interesting contribution, it is not the central thrust of this thesis as the 
main goal of this thesis is to investigate the effect of high frequency modes on the design of sliding mode controller.

In chapter 5 we then show that the full order system can be driven unstable and this is shown in Figure 5.2. This is an important result because the literature on sliding mode control discusses chattering but this is the first time that instability has been reported. Furthermore we demonstrate for the first time that the cause of instability depends on the slope of the sliding surface.

Then borrowing from some of the stability proofs published in the literature [25][30], we prove that for a range of the perturbation parameter $\varepsilon$, stability will result and the system will not suffer from chattering. The proof of stability required a new Lyapunov function to meet the sufficient conditions; this is discussed in section 5. It is then shown how the theoretical stability bound $\varepsilon^{*}$ is related to the slope of the sliding surface. This is shown in Figure 5.13.

Given that stability is related to the slope of the sliding surface we develop two new design procedures to select the sliding surface that will result in a stable sliding mode controller. Furthermore, the choice of the sliding surface will determine the bandwidth of the closed loop sliding mode controller.

In chapter 6 we propose using the $H^{\infty}$ control design method and the frequency domain to select a nonlinear sliding surface and demonstrate the usefulness of the method in example 6.1. Finally we use the technique of fuzzy logic control to design the sliding surface. This is discussed in chapter 7. 


\subsection{Literature Overview}

This thesis combines the idea of sliding mode control with singularly perturbed systems. Hence the literature in these two distinct research domains has been reviewed. There are only a few research articles that discuss the synergism achieved in combining these fields.

Sliding mode control first appeared in the Russian literature by Utkin [56] who established the theory and introduced the technique to the West. Since then, sliding mode control has been widely accepted as a powerful method to control uncertain nonlinear systems. Many research papers and books have been introduced in this field. Slotine [47][49] made an important contribution by applying a boundary layer around the sliding surface to decrease the chattering effects. Slotine [48] also had a good approach for using a sliding mode controller as a tracking controller with time varying boundary width. SiraRamirez [44][45] used integral control action to eliminate the chattering that appears in the sliding mode. Gao [19] introduced another approach to design the sliding mode controller called the reaching law approach.

The second field of investigation is the singular perturbation algorithm. Singular perturbation theory first appeared in mathematical literature then in control literature. Many books and research papers are found in this direction, the most famous are the books by Kokotovic [30], Khalil [28] and Nadiu [40].

Few researchers have applied sliding mode control on a system in singularly perturbed form. Slotine [50] proposed one of the earliest attempts by applying his sliding mode tracking control algorithm [47][49] to a robot in singular perturbed form. Heck [22] also introduced sliding mode control for a singular perturbed system. Heck [22] used two 
sliding mode controllers, one for each of the fast and slow subsystems. The reaching condition was investigated and was satisfied in her paper. Wu [60] discussed Heck's approach in more detail, and introduced a bound on the perturbed parameter $\varepsilon$ to ensure stability for the full order system. Dong [11] discussed the robustness properties of the two sliding mode controllers offered by Heck and suggested new conditions to design the sliding surface that ensure its robustness to disturbance.

The literature has proposed two methods to decouple the singularly perturbed system into slow and fast subsystems. One method is the Quasi steady state method, which is usually used to design feedback controllers for the full order system and the other is the diagonalization method that has reduced approximation error compared with the quasi steady state method. Furthermore, the diagonalization method has been used to design a single feedback controller as discussed by Cao [4].

Recently, stability analysis of sliding mode controllers for singularly perturbed systems has been discussed. Alvarez-Gallegos [1] introduced an approach to find a maximum value of the perturbation parameter $\varepsilon^{*}$ when two sliding mode controllers are used for the full order singularly perturbed system. Alvarez-Gallegos [1] uses the Lyapunov approach that was introduced by Kokotovic [30]. Innocenti [25][26] refined the Alvarez-Gallegos [1] approach by introducing a Lyapunov function that will ensure overall stability of the full order system.

Another area that has been discussed in this thesis is $H^{\infty}$ frequency shaped sliding mode control. Frequency shaped sliding mode control was first introduced by Gupta [21] to handle the flexibility problem in spacecraft. Young [61] introduces a more convenient form for frequency shaped sliding mode control. Nishimura [41] introduces an $H^{\infty}$ 
optimal control algorithm to design a frequency shaped sliding mode controller instead of a conventional linear quadratic regulator that has usually been used. $H^{\infty}$ frequency shaped sliding mode control showed superior performance compared with other methods of surface design for sliding mode control.

\subsection{Thesis Organization}

This Thesis consists of the following five chapters:

- Chapter 2 is a review of the sliding mode control technique and the most common methods used for design.

- Chapter 3 is a review of singular perturbation theory and its application to control.

- Chapter 4 is an overview for using a sliding mode controller for a singular perturbed system, including the two common design methods and the simulation results.

- Chapter 5 introduces the new proposed controller. The stability analysis for the proposed controller is shown. The interval for perturbation parameter $\varepsilon \in\left(0, \varepsilon^{*}\right)$ for the full order system is obtained where the closed loop system is asymptotically stable.

- Chapter 6 describes the frequency shaped method for the sliding surface and its benefits for the proposed controller.

- Chapter 7 investigates the single fuzzy sliding mode controller for the full order singularly perturbed system. 


\section{Chapter 2 Review of Sliding Mode Control}

\subsection{Introduction}

This chapter reviews three common design methods for a sliding mode controller. The mathematical steps associated with these methods are presented in such a manner that the reader can follow the formulation.

System model imprecision may come from actual uncertainty about the plant (e.g., unknown plant parameters), or from the choice of simplified representation of the systems dynamics. Modeling inaccuracies can be classified into two major kinds: structure (or parametric) uncertainties and unstructured uncertainties (or unmodeled dynamics). The first kind corresponds to inaccuracies in terms actually included in the model, while the second kind corresponds to inaccuracies in the system order.

Modeling inaccuracies can have strong adverse effects on nonlinear control systems. One of the most important approaches to deal with model uncertainty is robust control. There is currently a large interest in the variable structure control algorithms due to their robustness properties and possibility to decouple a high dimensional design problem into a set of lower dimensional independent sub-problems. In this chapter, brief reviews of two main design methods for sliding mode controllers are presented.

Variable structure control (VSC) is a variable switching feedback control, which means that the gain changes between two values. VSC uses switching control laws in order to drive the state trajectory of the system (usually a nonlinear plant) to a specific and user chosen surface in the state space (called the sliding or switching surface) and 
then keep the state trajectory on the surface. This surface is sometimes called the switching surface because if the state trajectory of the plant is "above" the surface the feedback gain will have a value which is different from the other gain when the state trajectory is "below" the switching surface using a control law that is discontinuous across the switching surface.

The switching surface is referred to as the sliding surface because when the state trajectory reaches this surface it stays on this surface (slides down or up) until it reaches an equilibrium point. The main principles for switching control theory can be explained by a simple example. In this example we will explain how two unstable systems can be combined using a switching strategy to obtain a stable system.

Consider a second order plant in companion form that takes the form, DeCarlo [10]

$$
\begin{aligned}
& {\left[\begin{array}{l}
\dot{x}_{1} \\
\dot{x}_{2}
\end{array}\right]=\left[\begin{array}{ll}
0 & 1 \\
1 & 2
\end{array}\right]\left[\begin{array}{l}
x_{1} \\
x_{2}
\end{array}\right]+\left[\begin{array}{l}
0 \\
1
\end{array}\right] u,} \\
& y=\left[\begin{array}{ll}
1 & 0
\end{array}\right]\left[\begin{array}{l}
x_{1} \\
x_{2}
\end{array}\right] .
\end{aligned}
$$

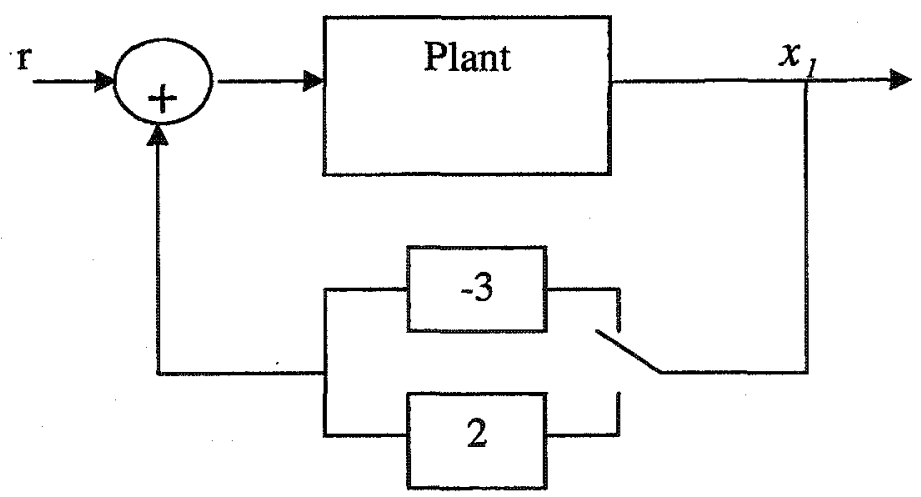

Figure 2.1: Block diagram of switching feedback [10]

The control law $u$ takes the form,

$u=k\left(x_{1}\right) x_{1}$, 
where $x_{1}$ is the plant output. The gain $k\left(x_{1}\right)$ can take on one of two values, "2" or "-3". This system is illustrated in Figure 2.1, which has two linear structures, one for $k\left(x_{1}\right)=2$ and the other for $k\left(x_{1}\right)=-3$. With the switch in the upper position, $k\left(x_{1}\right)=-3$, the closed loop system takes the form

$\left[\begin{array}{l}\dot{x}_{1} \\ \dot{x}_{2}\end{array}\right]=\left[\begin{array}{cc}0 & 1 \\ -2 & 2\end{array}\right]\left[\begin{array}{l}x_{1} \\ x_{2}\end{array}\right]$,

while with the switch in the lower position, $k\left(x_{1}\right)=2$, the closed loop system will be $\left[\begin{array}{l}\dot{x}_{1} \\ \dot{x}_{2}\end{array}\right]=\left[\begin{array}{ll}0 & 1 \\ 3 & 2\end{array}\right]\left[\begin{array}{l}x_{1} \\ x_{2}\end{array}\right]$.

In both cases our system is unstable. In the upper case $\left(k\left(x_{1}\right)=-3\right)$ the equilibrium point $(0,0)$ has an unstable focus as shown in Figure 2.2(b), while in the lower case $\left(k\left(x_{1}\right)=2\right)$ the equilibrium point becomes a saddle point as shown in Figure 2.2(a). Switching occurs with respect to the sliding or switching surface denoted by $S=0$. For example consider $S=\lambda x_{1}+x_{2}$ with $\lambda>1$, and the feedback switching law

$$
k\left(x_{1}\right)= \begin{cases}-3 & \text { If } S(x) x_{1}>0 \\ 2 & \text { If } S(x) x_{1}<0\end{cases}
$$

Thus sliding mode control can combine the two unstable systems along the switching surface to obtain a stable closed loop system as illustrated in Figure 2.2(c)

From this simple example it is seen that the most important task is to design the switched control law that will drive the plant state to the switching surface and maintain it there. Usually a Lyapunov approach is used to achieve this task. 


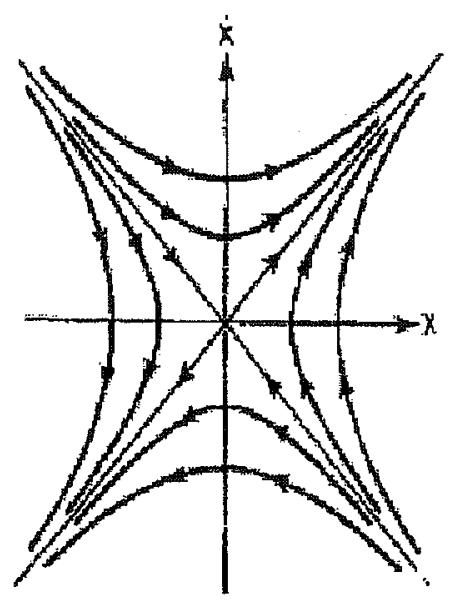

Suddle Pajnit

(a)

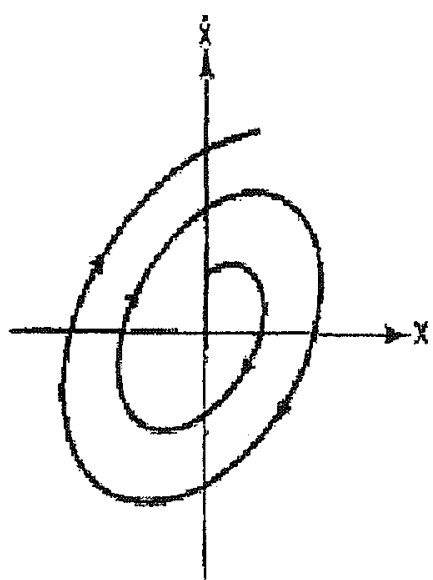

Uabtalle racus

(b)

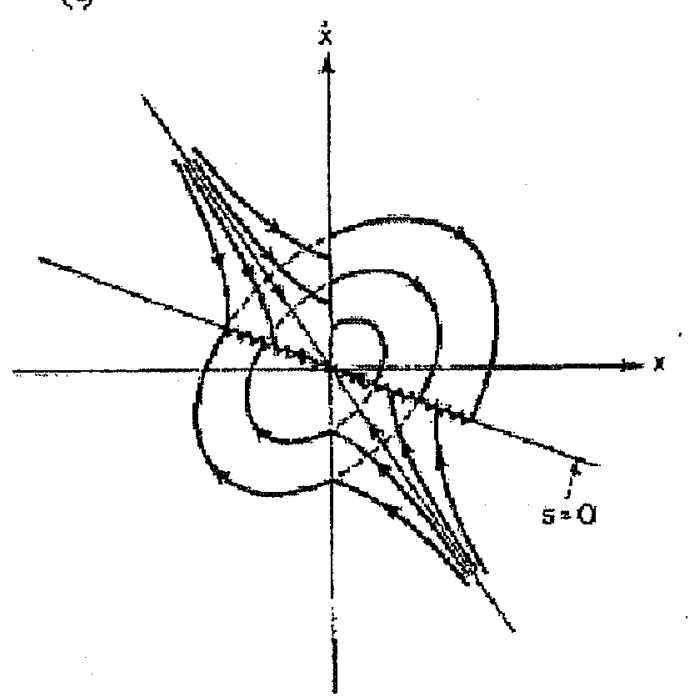

(c)

Figure 2.2: Phase plane plots for the switched feedback plant [10][56]

A generalized Lyapunov function that characterizes the motion of the state trajectory to the sliding surface is defined in terms of the surface. For each chosen switched control structure, one chooses the "gains" so that the derivative of this Lyapunov function is negative definite, thus guaranteeing motion of the state trajectory to the surface. After proper design of the surface, a switched controller is constructed so that the tangent vectors of the state trajectory point towards the surface so that the state is driven to the 
sliding surface and is maintained on it. Such a controller results in discontinuous closed loop systems. The two main design methods that are discussed here are the equivalent control method offered by Utkin [55][56] and the tracking control method offered by Slotine [48][49].

\subsection{Equivalent control method}

The design of a sliding mode controller with this method breaks down into two main steps:

1. Determine the switching surface so that when the system is restricted to the switching surface the desired behavior is produced; and

2. Construct switched feedback gains that will drive the state trajectory to the sliding surface and maintain it there.

\subsubsection{Sliding surface design}

Before designing the switching surface it is useful to note that VSC produces system dynamics with discontinuous right hand side due to the switching action of the controller as will be shown in the next step. Thus the equations describing the dynamics of the system fail to satisfy conventional existence and uniqueness theory [56].

One of the straightforward and mostly used methods to solve this problem is the method of equivalent control as proposed by Utkin [55] and Drakunov [14]. In this method the system equation in (2.5) is solved by using regularization to replace the initial problem by a closely similar one for which familiar methods can be used.

Consider a system depending linearly on the control

$\dot{x}=f(x, t)+B(x, t) u$ 


$$
u_{i}= \begin{cases}u_{i}^{+}(x, t) & \text { if } s_{i}(x, t)>0 \\ u_{i}^{-}(x, t) & \text { if } s_{i}(x, t)<0\end{cases}
$$

where $u_{i}$ is the $\mathrm{i}^{\text {th }}$ component of $u$ and $s_{i}(x, t)$ is the $\mathrm{i}^{\text {th }}$ component of $s(x, t) \in R^{m}$, and $u^{-}(\boldsymbol{x}, t)$ and $u^{+}(\boldsymbol{x}, t)$ are continuous functions. The control $u(x, t)$ undergoes a discontinuity on the surface $s(x, t)=0$, which is called the switching surface.

The state vector $x(t) \in R^{n}$ and control vector $u(t) \in R^{m}$, thus $f(x, t) \in R^{n}$ and $B(\boldsymbol{x}, t) \in R^{n \times m}$. Assume that the linear switching surface $s(\boldsymbol{x}, t)$ has the general form $s(x, t)=C x(t)$ (for simplicity $s(x, t)=S$ ) where $C \in R^{m \times n}$, so $S \in R^{m}$ and $\frac{\partial S}{\partial x}=C$. We will consider, without lost of generality, the system given by equation (2.5) as a single input single output system. This means that $C \in R^{I \times n}$ and $S \in R$. Suppose that at time $t_{0}$, the system state reaches the switching surface and that the sliding mode exists for $t \geq t_{0}$. The existence of the sliding mode means that $S=0$ and $\dot{S}=0$. Differentiating $S$ along the trajectory of $\boldsymbol{x}$ gives

$$
\dot{S}=\frac{\partial S}{\partial x} \dot{x}=\frac{\partial S}{\partial x}\left[f(x, t)+B(x, t) u_{e q}\right]=0
$$

where $u_{e q}$ is the equivalent control that solves equation (2.7). Assume that the matrix product $\frac{\partial S}{\partial x} B(x, t)$ is nonsingular, then from equation (2.7) we have

$$
u_{e q}=-\left[\left[\frac{\partial S}{\partial x} B(x, t)\right]^{-1} \frac{\partial S}{\partial x} f(x, t)\right] .
$$

The dynamics of system (2.5) on the switching surface for $t \geq t_{0}$ is given by substituting $u=u_{e q}$ in equation (2.5) and gives 


$$
\dot{x}=\left[I-B(x, t)\left[\frac{\partial S}{\partial x} B(x, t)\right]^{-1} \frac{\partial S}{\partial x}\right] f(x, t)
$$

and since we assume that $S$ is a linear switching surface with respect to $\boldsymbol{x}$, and substituting $S=C x$ (where $\frac{\partial S}{\partial x}=C$ ) into equation (2.9) then

$$
\dot{\boldsymbol{x}}=\left[I-B(\boldsymbol{x}, t)[C B(\boldsymbol{x}, t)]^{-1} C\right] f(\boldsymbol{x}, t) .
$$

Determining the sliding surface parameter $C$ that will satisfy the desired dynamic equation in the sliding mode is possible by using equation (2.10). If $f(x, t)$ is linear and in the desirable canonical form then one can determine the surface parameters $C$ by solving equation (2.10) for the specific closed loop characteristic equation. If $f(x, t)$ is not in the desirable form, many methods to obtain the surface parameter $C$ can be found. The most famous method is to transform $f(x, t)$ to a more general form called the regular form. The regular form of the plant dynamics is

$$
\begin{aligned}
& \dot{x}_{1}=f_{I}(\boldsymbol{x}, t) \\
& \dot{x}_{2}=f_{2}(\boldsymbol{x}, t)+B_{2}(\boldsymbol{x}, t) u,
\end{aligned}
$$

where $x_{1} \in R^{n-m}$ and $x_{2} \in R^{m}$. A system in this form allows us to compute the surface parameter $C$ based on the desired reduced order dynamic (n-m) equation. Detailed information about the transformation procedure can be found in El-Ghezawi [15] in the case of a linear system or Hunt [24], Luk'yanov [39] in the case of a nonlinear system.

\subsubsection{Controller design}

The controller design is the second phase of the SMC design. The purpose of this phase is to determine the switching feedback gains that will drive the state trajectory to 
the sliding surface and maintain it there. This can be done by using a Lyapunov stability analysis method to satisfy the reaching condition. Without loss of generality consider the system in equation (2.5) as a single input system (where the design can extend easily to multi input systems). Choose a Lyapunov function [14]

$V(x)=\frac{1}{2} S^{2}$

where $S=C x, C \in R^{I \times n}$. For stability it is required that the derivative of $V(x)$ be negative, therefore, $\dot{V}=S \dot{S}<0$. This can be done if $S \dot{S} \leq-\eta|S|$ where $\eta$ is an arbitrary positive constant. Let $S \dot{S}=-\eta|S|$ then $\dot{S}=-\eta \operatorname{sgn}(S)$ where $\operatorname{sgn}(S)$ is the signum function,

$$
\operatorname{sgn}(S)= \begin{cases}1 & \text { If } S>0 \\ -1 & \text { If } S<0\end{cases}
$$

since $S=C x$,

$\dot{S}=C \dot{x}=C[f(x, t)+B(x, t) u]=-\eta \operatorname{sgn}(S)$.

Then one can solve equation (2.13) for the control signal $u$ as,

$u=-[C B]^{-1} C f-[C B]^{-1} \eta \operatorname{sgn}(S)$,

where for simplicity we consider $f(x, t)=f$ and $B(x, t)=B$. Recognizing from equation (2.8) that $u_{e q}=-[C B]^{-1} C f$ then equation $(2.14)$ can be rewritten as, $u=u_{e q}-k \operatorname{sgn}(S)$

where the gain $k=[C B]^{-1} \eta$.

Equation (2.15) indicates that the control law is the sum of two terms. The first term is nonlinear state feedback and it is known from the previous section that it keeps the states on the sliding surface. The second term is a switching control term and this ensures 
that the states reach the sliding surface. The choice of gain $k$ can be obtained from equation (2.14).

\subsection{Reaching law approach}

Gao [19] proposed a reaching law, which directly specifies the dynamics of the switching surface by the differential equation

$\dot{S}=-Q \operatorname{sgn}(S)-K h(S)$

where the gains $Q$ and $K$ are diagonal matrices with positive elements, $\operatorname{sgn}(S)=\left[\operatorname{sgn}\left(s_{1}\right) \ldots . \operatorname{sgn}\left(s_{m}\right)\right]^{T}, h(S)=\left[h_{1}\left(s_{1}\right) \ldots . h_{m}\left(s_{m}\right)\right]^{T}$ and the scalar functions $h_{i}$ satisfy the conditions [19], $S_{i} h_{i}\left(S_{i}\right)>0$, when $S_{i} \neq 0$

Various choices of $Q$ and $K$ specify different rates for approaching $S=0$ and yield different structures in the reaching law. Similar to the equivalent control method, the control law can be directly obtained by computing the time derivative of $S$ along the reaching mode trajectory, i.e.,

$\dot{S}=C(f(x, t)+B(x, t) u)=-Q \operatorname{sgn}(S)-K h(S)$

Thus, we have

$$
u=-[C B]^{-1}(C f(x, t)+Q \operatorname{sgn}(S)+K h(S))
$$

By this approach, the resulting sliding mode is not preassigned but follows a natural trajectory on the first reach first switch scheme [19]. The switching takes place depending on the location of the initial state.

Three practical forms of the reaching law are: 
- Constant rate reaching where, $\dot{S}=-Q \operatorname{sgn}(S)$. The state reaches the switching surface $S=0$ at a constant rate. The resulting control is the same as the equivalent control law given by equation (2.15).

- Constant plus proportional rate reaching where, $\dot{S}=-Q \operatorname{sgn}(S)-K S$. The term $-K S$ forces the state to approach the switching surfaces faster when $S$ is large. The resulting controller is the same as the control (2.15) but with a linear feedback control term added to the switching part.

- Power rate reaching where, $\dot{S}=-K\left|S_{i}\right|^{\alpha} \operatorname{sgn}(S)$ where $0<\alpha<1$. In this case, the reaching speed is faster when the state is far away from $S$, and slower when the state is near $S$.

\subsection{Robustness and invariance}

One of the most distinguishing properties of a sliding mode controller is the robustness and insensitivity to modeling errors and disturbances. The sliding mode is said to be robust if the differential equation of the sliding mode is entirely independent of the effects related to modeling uncertainties and disturbances. This is true providing that the so called matching condition, which is characterized in the following is met.

Consider first, a linear uncertain system

$\dot{x}=(A+\Delta A) x+(B+\Delta B) u+E f$

where $x \in R^{n}, \quad u \in R^{m}, A, B$ are nominal matrices of the system, $\Delta A, \Delta B$ denote uncertain components and $E$ denotes a disturbance matrix and $f$ is an external disturbance. If the condition 
$\operatorname{rank}([B: \triangle A: \Delta B: E])=\operatorname{rank}(B)$

is satisfied, then the matching condition holds [15]. The matrix ([B: $\triangle A: \triangle B: E])$ is the concatenation of the column space. Therefore when condition (2.20) is met this means that $\triangle A, \Delta B$ and $E$ are restricted to the space spanned by the columns of $B$. The physical meaning of equation (2.20) is that all uncertainties and disturbances enter the system through the control channel. The matching conditions can also be extended to general nonlinear systems of the form Gao [19],

$\dot{x}=(f(x)+\Delta f(x, p, t))+(B(x, t)+\Delta B(x, p, t)) u+F(x, p, t)$

where $p$ is an uncertain parameter vector whose values belong to some bounded set. Hence $\Delta f(x, p, t)$ and $\Delta B(x, p, t)$ represent the variations and uncertainties in the plant parameters and control interface respectively, and $F(x, p, t)$ represents the lumped disturbances. Similar to the linear system, the invariance holds if the following matching conditions are satisfied:

$$
\begin{aligned}
& \Delta f(x, p, t)=B(x, t) \Delta \tilde{f}(x, p, t) \\
& \Delta B(x, p, t)=B(x, t) \Delta \tilde{B}(x, p, t) \\
& F(x, p, t)=B(x, t) \Delta \tilde{F}(x, p, t)
\end{aligned}
$$

This means that the plant uncertainties $\Delta f$ and $\Delta B$ are required to lie in the image space of $B(x, t)$ for all values of $x$ and $t$, DeCarlo [10].

\subsection{Chattering reduction}

The sliding mode controllers require an infinitely fast switching mechanism. However, due to physical limitations in real world systems, directly applying the above 
developed control algorithm will always lead to oscillations in some vicinity of the switching surface [48]. This oscillation is called chattering. If the switching gain is large such chattering may cause unpredictable instability. The chattering problem is considered as a major drawback for sliding mode control. To reduce chattering many methods are implemented such as the smoothing function method [3] and the boundary layer method [48] which is discussed later in this chapter.

\subsubsection{Smoothing function method}

The smoothing function is a means for eliminating chattering while trying to preserve good robustness properties around the sliding surface. There is a large class of smoothing functions available to convert the discontinuous control laws. One of them, which is used for SISO (single input single output) systems, has the form [3],

$$
\theta(s)=\frac{s}{s+\delta}
$$

where $\delta>0$ is chosen as a small positive constant. This can be extended to MMMO (multi input multi output) systems and $\theta(s)$ in this case has the form,

$$
\theta_{i}(s)=\frac{s_{i}}{\|s\|+\delta_{i}} \quad i=1,2, \ldots m
$$

This will only effect the second term in the control law given by equation (2.15). The new control law can be rewritten as,

$$
u=u_{e q}-K \theta
$$

where $\theta=\left[\begin{array}{lll}\theta_{1} & \ldots & \theta_{m}\end{array}\right]$ replaces $\operatorname{sgn}(s)$ in equation $(2.15)$. Thus in the region around the sliding surface determined by the value of $\delta$, the system is effectively a high gain 
feedback system and possesses the properties of certain classes of disturbance rejection and parameter invariance [3].

\subsection{Tracking control method}

The aim of this method is to achieve accurate tracking for a class of nonlinear systems in the presence of disturbances and parameter variations. Consider the single input dynamic system [47][48][49].

$x^{n}=f(x, t)+b(x, t) u$

The scalar $x$ is the output of interest, $u$ is the control input, $x=\left[x \dot{x} \ldots \ldots x^{n-1}\right]^{T}$ is the state vector and the system is in controllable canonical form where $x^{n}$ denotes the $\mathrm{n}^{\text {th }}$ time derivative of $x$. The term $f(x, t)$ is not exactly known but is upper bounded by a known continuous function of $\boldsymbol{x}$. The control gain $b(\boldsymbol{x}, t)$ is not exactly known but is bounded by a known function of $\boldsymbol{x}$ and with known sign. The task is to get the state $\boldsymbol{x}$ to track the specific desired time varying state $x_{d}=\left[x_{d} \dot{x}_{d} \ldots x_{d}^{n-1}\right]^{T}$ in the presence of model imprecision on $f(x, t)$ and $b(x, t)$ using control $u$. Define the state error $\tilde{\boldsymbol{x}}$ where $\tilde{x}=x-x_{d}=\left[\tilde{x} \dot{\tilde{x}}_{0 . \ldots} \tilde{x}^{n-1}\right]^{T}$. A time varying surface $S(t)$ is defined in the state space $R^{n}$ as,

$S(t): s(x, t)=0$

where $s(x, t)=\left(\frac{d}{d t}+\lambda\right)^{n-1} \tilde{x}$

Here $\lambda$ is a strictly positive constant, taken to be the bandwidth of the system and it will be chosen later. The problem of tracking $x=x_{d}$ is now equivalent to remaining on 
the surface $S(t)$ for all $t>0$. This means that $s(x, t)=0$. To derive the control law $u$ for such a task a Lyapunov-like function is defined as

$$
V=\frac{1}{2} s^{2} \quad(s=s(x, t))
$$

with $V>0$ for $s>0$. A sufficient condition for the states to converge to the surface $S(t)=0$ is,

$\dot{V}=\frac{1}{2} \frac{d}{d t} s^{2} \leq-\eta|s|$

which can be rewritten as,

$s \dot{s} \leq-\eta|s|$

since $\frac{s}{|s|}=\operatorname{sgn}(s)$ then,

$\dot{s} \operatorname{sgn}(s) \leq-\eta$

where $\eta$ is an arbitrary positive constant that will ensure $\tilde{x} \rightarrow 0$ as $t \rightarrow \infty$. The next step is to compute $\dot{s}$ from (2.26),

$$
\dot{s}=x^{n}-x_{d}^{n}+\sum_{k=1}^{n-1}\left(\begin{array}{c}
n-1 \\
k
\end{array}\right) \lambda^{k} \tilde{x}^{n-k}
$$

Substituting the value of $x^{n}$ given by equation (2.25) into equation (2.31) gives,

$$
\dot{s}=f(x, t)+b(x, t) u-x_{d}^{n}+\sum_{k=1}^{n-1}\left(\begin{array}{c}
n-1 \\
k
\end{array}\right) \lambda^{k} \tilde{x}^{n-k}
$$

The value of $\dot{s}$ from equation (2.32) is used to rewrite equation (2.30) as follows,

$$
\operatorname{sgn}(s)\left(f(x, t)+b(\boldsymbol{x}, t) u-x_{d}^{n}+\sum_{k=1}^{n-1}\left(\begin{array}{c}
n-1 \\
k
\end{array}\right) \lambda^{k} \tilde{x}^{(n-k)}\right) \leq-\eta
$$


Defining a boundary for the gain let, $b_{\min } \leq b \leq b_{\max }$ where for simplicity $b(x, t)=b$. Let the estimated gain be $\hat{b}=\left(b_{\min } b_{\max }\right)^{0.5}$, Slotine [48]. The estimated function $\hat{f}$ is known for our model. The estimation error on $f$ is assumed to be bounded by some known function $F=F(x)$,

$|\hat{f}-f| \leq F$

The best approximation for control $u$ that will achieve $\dot{s}=0$ can be obtained from equation (2.32) as

$\hat{u}=\hat{b}^{-1}\left[-\hat{f}(x, t)+x_{d}^{n}-\sum_{k=1}^{n-1}\left(\begin{array}{c}n-1 \\ k\end{array}\right) \lambda^{k} \tilde{x}^{(n-k)}\right]$

In order to satisfy the sliding condition (2.28) despite uncertainty of the dynamics $f(x, t)$, we add to $\hat{u}$ a term discontinuous across the surface $s=0$. The control law is defined as,

$u=\hat{u}-\hat{b}^{-1} k \operatorname{sgn}(s)$

where $\operatorname{sgn}(s)$ is the signum function. Using the value of $u$ represented by equation (2.36) in the expression of $\dot{s}$ given by equation (2.32) leads to,

$\left.\dot{s}=f+b \hat{b}^{-1}\left[-\hat{f}+x_{d}^{n}-\sum_{k=1}^{n-1}\left(\begin{array}{c}n-1 \\ k\end{array}\right) \lambda^{k} \tilde{x}^{(n-k)}-k \operatorname{sgn}(s)\right]-x_{d}^{n}+\sum_{k=1}^{n-1}\left(\begin{array}{c}n-1 \\ k\end{array}\right) \lambda^{k} \tilde{x}^{(n-k)}\right)$

which can be rewritten as,

$\dot{s}=\left(f-b \hat{b}^{-1} \hat{f}\right)+\left(1-b \hat{b}^{-1}\right)\left(-x_{d}^{n}+\sum_{k=1}^{n-1}\left(\begin{array}{c}n-1 \\ k\end{array}\right) \lambda^{k} \tilde{x}^{(n-k)}\right)-b \hat{b}^{-1} k \operatorname{sgn}(s)$

To satisfy the sliding condition given by equation (2.30) and using the expression of $\dot{s}$ given by equation (2.37) such that, $\dot{s} \operatorname{sgn}(s) \leq-\eta$, then we get the inequality, 


$$
\left[\left(f-b \hat{b}^{-1} \hat{f}\right)+\left(1-b \hat{b}^{-1}\right)\left(-x_{d}^{n}+\sum_{k=1}^{n-1}\left(\begin{array}{c}
n-1 \\
k
\end{array}\right) \lambda^{k} \tilde{x}^{(n-k)}\right)\right] \operatorname{sgn}(s)-b \hat{b}^{-1} k \leq-\eta
$$

The above inequality is always true if

$$
b \hat{b}^{-1} k \geq\left|\left(f-b \hat{b}^{-1} \hat{f}\right)+\left(1-b \hat{b}^{-1}\right)\left(-x_{d}^{n}+\sum_{k=1}^{n-1}\left(\begin{array}{c}
n-1 \\
k
\end{array}\right) \lambda^{k} \tilde{x}^{(n-k)}\right)\right|+\eta
$$

then $k$ must satisfy

$k \geq\left|\left(\hat{b} b^{-1} f-\hat{f}\right)+\left(\hat{b} b^{-1}-1\right)\left(-x_{d}^{n}+\sum_{k=1}^{n-1}\left(\begin{array}{c}n-1 \\ k\end{array}\right) \lambda^{k} \tilde{x}^{(n-k)}\right)+\eta \hat{b} b^{-1}\right|$

The control laws that satisfy the sliding condition are discontinuous across the surface $S(t)$ thus leading to control chattering. In general, chattering must be eliminated for the control to perform properly. This can be achieved, in this method, by using a boundary layer, $J(t)$, neighboring the switching surface as shown in Figure 2.3, and given by

$$
J(t)=\{x,|s(x, t)| \leq \phi\}, \quad \phi>0
$$

This is achieved by choosing the control law $u$ outside $J(t)$ as before and then interpolating $u$ inside $J(t)$. As mentioned before $s(x, t)$ can be directly translated into bounds on the tracking vector error $\tilde{x}$ as can be seen in the equation (2.26). For the tracking task to be achieved using a finite control $u$, the initial desired state $x_{d}(0)$ must be such that,

$$
x_{d}(0)=x(0)
$$




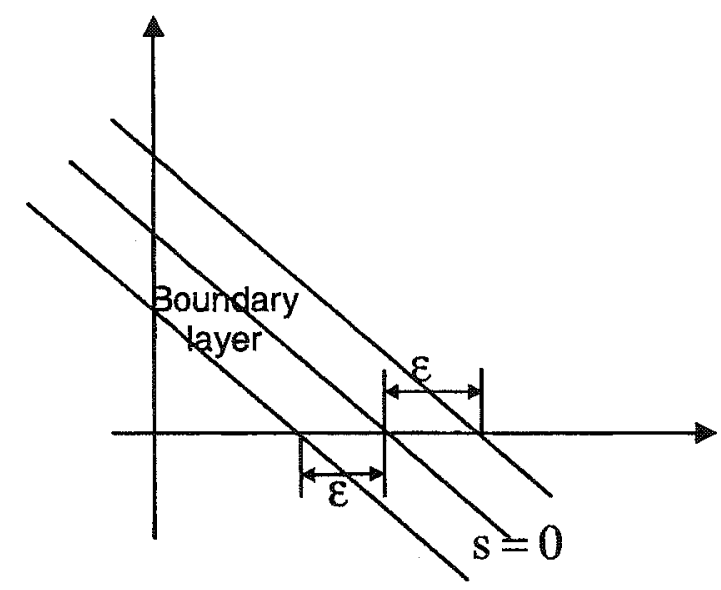

Figure 2.3 : The construction of the boundary layer in the case that $n=2$

Given the initial condition (2.41), the problem of tracking $x=x_{d}$ is equivalent to remaining on the surface $S(t)$ for all $t>0$. This means that $s=0$ represents a linear differential equation whose unique solution is $\tilde{x}=0$ [47]. Since $s(x, t)=\left(\frac{d}{d t}+\lambda\right)^{n-1} \tilde{x}$ then the tracking error $\tilde{x}$ is obtained through a sequence of low pass filters from $s$

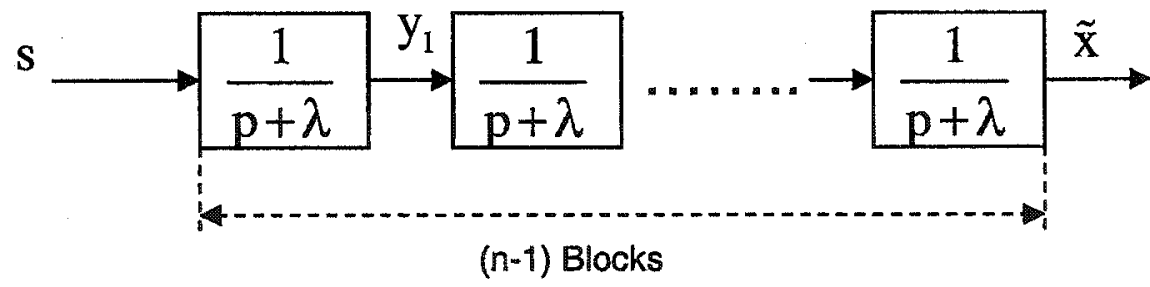

Figure 2.4: Lowpass filter blocks [47]

From Figure 2.4 ( $\mathrm{p}$ is the Laplace operator) the output of the first filter will be

$$
y_{1}=\int_{0}^{t} e^{-\lambda(t-T)} s(T) d T \text {. Let } \forall t \geq 0 \quad|s(\mathbf{x}, t)| \leq \phi
$$

Then $\left|y_{1}\right| \leq \phi \int_{0}^{t} e^{-\lambda(t-T)} d T=\frac{\phi}{\lambda}\left(1-e^{-\lambda t}\right) \leq \frac{\phi}{\lambda}$ 
Apply the same for the second filter and so on until we have

$$
|\tilde{x}| \leq \frac{\phi}{\lambda^{n-1}}=\varepsilon
$$

From the previous result the value of $\phi$ can be determined based on the required precision. This method for obtaining $\phi$ is usually called the guaranteed precision method [48].

\subsubsection{Robustness to uncertainties}

We will now investigate the trajectory inside the boundary layer $\phi$. This will allow us to understand the relationship between the robustness of the sliding mode controller, its bandwidth and the tracking precision. Consider the case of no uncertainty in the gain $b$. To simplify the analysis, for a nonlinear system, let us take $b=1$ so that $x^{n}=f(x, t)+u$

where $f(x, t)=\hat{f}(x, t)+\Delta f(x, t)$ and $\hat{f}(x, t)$ is the available model of $f(x, t)$. We assume $|\Delta f(x, t)| \leq F(\boldsymbol{x}, t)$. The sliding surface can be defined as before in equation (2.36). Thus the control law takes the form,

$u=-\hat{f}(x, t)-\sum_{k=1}^{n-1}\left(\begin{array}{c}n-1 \\ k\end{array}\right) \lambda^{k} \tilde{x}^{(n-k)}-k \operatorname{sgn}(s)$.

Since there is no uncertainty in the gain, this means $\hat{b} b^{-1}=1$, then the value of $k$ in equation (2.39) can be defined as follows,

$$
k=F(x, t)+\eta
$$

Using the saturation function instead of the signum function equation (2.46) can be rewritten as 
$u=-\hat{f}(x, t)-\sum_{k=1}^{n-1}\left(\begin{array}{c}n-1 \\ k\end{array}\right) \lambda^{k} \tilde{x}^{(n-k)}-k \operatorname{sat}\left(\frac{s}{\phi}\right)$

From the definition of the sliding surface given by the equation (2.26) then $\dot{s}$ can be defined as follows,

$$
\dot{s}=x^{n}-x_{d}^{n}+\sum_{k=1}^{n-1}\left(\begin{array}{c}
n-1 \\
k
\end{array}\right) \lambda^{k} \tilde{x}^{(n-k)} .
$$

Substituting the value of $x^{n}$ given by equation (2.45) in equation (2.49) gives us,

$$
\dot{s}=f(x, t)+u-x_{d}^{n}+\sum_{k=1}^{n-1}\left(\begin{array}{c}
n-1 \\
k
\end{array}\right) \lambda^{k} \tilde{x}^{(n-k)}
$$

Substituting the value of $u$ from equation (2.48) and using equation (2.44) the expression of $\dot{s}$ in equation (2.50) can be rewritten as,

$$
\begin{array}{r}
\dot{s}=f(\boldsymbol{x}, t)-\hat{f}(\boldsymbol{x}, t)-\sum_{k=1}^{n-1}\left(\begin{array}{c}
n-1 \\
k
\end{array}\right) \lambda^{k} \tilde{x}^{(n-k)} \\
-k \operatorname{sat}\left(\frac{s}{\phi}\right)-x_{d}^{n}+\sum_{k=1}^{n-1}\left(\begin{array}{c}
n-1 \\
k
\end{array}\right) \lambda^{k} \tilde{x}^{(n-k)}
\end{array}
$$

Inside the boundary layer we can write the expression of $\dot{s}$ as

$$
\dot{s}=-k\left[\frac{s}{\lambda^{n-1} \varepsilon}\right]+\Delta f(x, t)-x_{d}^{n}
$$

From equation (2.52) we see that the variable $s$ (the distance to the surface $S(t)$ ) is the output of a stable first order filter. The input to this filter is $\Delta f(x, t)$ and $-x_{d}^{n}$. From Figure 2.5 it is clear that perturbations are low pass filtered according to equation (2.52). Let $k\left(x_{d}, t\right)$ be upper bounded say by $k_{\max }$, so $\frac{k_{\max }}{\lambda^{n-1} \varepsilon}$ can be seen as the maximum 
break frequency of the dynamic filter. On the other hand, $\lambda$ is supposed to be chosen as small with respect to the unmodeled high frequency dynamics.

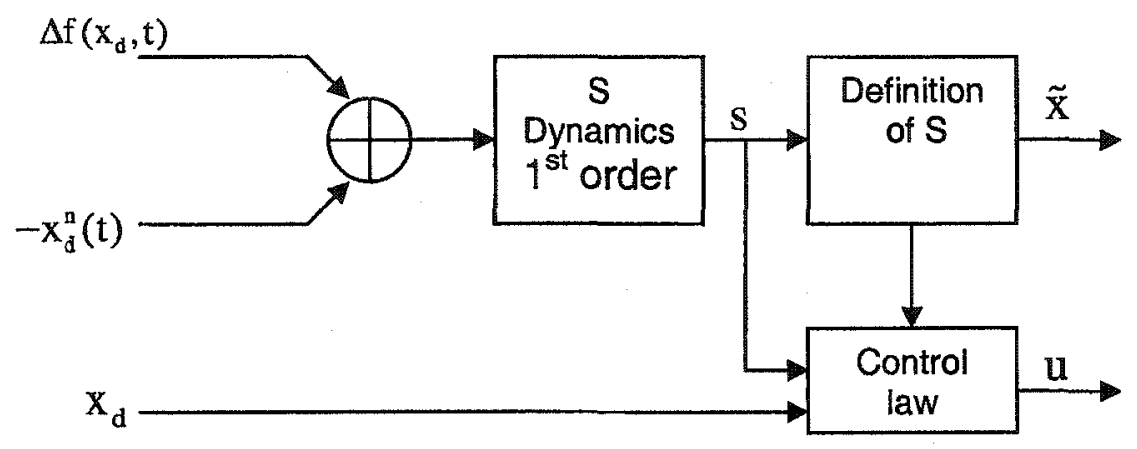

Figure 2.5: Dynamic structure of the controlled system

Thus if we set $\lambda$ to be the largest acceptable break frequency based on the definition of the surface $s$ then $\frac{k_{\max }}{\lambda^{n-1} \varepsilon} \leq \lambda$. This will get the best attainable tracking precision $\varepsilon$ where,

$\lambda^{n} \varepsilon=k_{\max }$

which is referred to as the balance condition [48]. In the case where the gain has uncertainty (i.e. $\hat{b} b^{-1} \neq 1$ ), then the system will have the form

$x^{n}=f(x, t)+b(x, t) u$.

Then the balance condition can be written as

$\lambda^{n} \varepsilon=\frac{\hat{b}}{b} k_{\text {max }}$

The balance condition can be another method for choosing the boundary layer thickness $\phi$. The shortcoming of using the boundary layer is that the robustness properties of the sliding mode are actually lost inside the boundary layer Decarlo [10], 
such that uncertainties and parasitic dynamics must be carefully considered and modeled in the feedback design.

\subsection{Chapter summary}

In the tracking control method proposed by Slotine [48][49], the problem of choosing the slope of the sliding surface " $\lambda$ " (or bandwidth of the controller) is limited by certain factors. The most important is the unmodeled high frequency dynamics that are frequently associated with the structural resonant modes. In this case Slotine suggests that $\lambda$ be smaller than the frequency of the lowest unmodeled high frequency dynamic mode. However an analysis of the effect of the sliding surface shape on the high frequency modes is seldom done.

In this chapter we have reviewed well-known methods for sliding mode control design. These methods are:

- The equivalent control method offered by Utkin [54][55]. It is well known by its direct approach and simplicity.

- Slotine's [48][49] method has also been discussed and its advantage when using it in tracking control has been highlighted. The surface parameter selection in this method is different than other methods and the selection depends on the unmodeled high frequency dynamics.

- The reaching control law approach has been reviewed and the main difference between this method and the equivalent control method has been discussed. 
These three methods are used in the following chapters when we design sliding mode controllers for singularly perturbed systems. The equivalent control method is our main approach to design the sliding mode controllers. Slotine's approach is used when we discuss the effect of the unmodeled high frequency dynamics. The reaching law approach is used when we discuss the stability analysis for the closed loop system. 


\section{Chapter 3 The Singular Perturbation Method}

The main objective of this chapter is to familiarize the reader with the concept of a singularly perturbed system.

\subsection{Definition and Features}

A basic problem in control system theory is the mathematical modeling of physical systems. The realistic representation of many systems calls for high order differential equations. The presence of some parasitic parameters such as masses, moments of inertia, resistances, inductances and capacitances are often the cause of increased order of these systems. Broadly speaking, a system where the suppression of small parameters is responsible for degeneration of dimension is termed a "singularly perturbed" system. In other words, a problem described by a differential equation involving a small parameter $\varepsilon$ is called a singular perturbation problem if the order of the differential equation becomes lower for $\varepsilon=0$ than for $\varepsilon \neq 0[30]$.

The analysis and control of large scale systems has always been a complicated task, not only due to the high order nature but also due to the fact that the majority of these systems possess interacting dynamic phenomena of widely different speeds [31]. This gives rise to widely separated groups of eigenvalues. In practice, we come across many systems that are subject to the two-time scale phenomenon. For example, robot manipulators having flexible links and flexible joints [12]. Large spacecraft with flexible structures are another 
example. Singular perturbation is seen as good modeling approach for these types of systems.

Consider a linear time invariant finite dimensional singularly perturbed system [40].

$\dot{x}_{1}(t)=A_{1 I} x_{1}(t)+A_{12} x_{2}(t)+B_{1} u(t)$

$\varepsilon \dot{x}_{2}(t)=A_{21} x_{1}(t)+A_{22} x_{2}(t)+B_{2} u(t)$

where $x_{l} \in R^{m}$ and $x_{2} \in R^{n}$ are the state vectors, $u \in R^{r}$ is the control vector. The matrices $A_{i j}$ and $B_{i}$ are of appropriate dimensions and $\varepsilon$ is a small positive parameter. In the next section, two different approaches for the decomposition of the singularly perturbed system are considered, which are the quasi steady state approach and block diagonalization [30][40][51].

\subsection{Quasi steady state method}

Due to the occurrence of the slow and fast modes in the system given by equations (3.1) and (3.2) respectively, the fast modes are important only during a short initial period. After that period they are negligible, and the behavior of the system can be described by its slow modes. The model with fast modes neglected is called the quasi steady state model [30]. Neglecting the fast modes is equivalent to assuming that they are infinitely fast, that is, letting $\varepsilon \rightarrow O$ in equation (3.2). Without the fast modes the system described by equations (3.1) and (3.2) reduces to

$$
\begin{aligned}
& \hat{\tilde{x}}_{1}=A_{11} \tilde{x}_{1}+A_{12} \tilde{x}_{2}+B_{1} \tilde{u}, \\
& 0=A_{21} \tilde{x}_{1}+A_{22} \tilde{x}_{2}+B_{2} \tilde{u}
\end{aligned}
$$

where the symbol " $\sim$ indicates that $\varepsilon=0$. When $A_{22}$ is nonsingular, we can express $\tilde{x}_{2}$ as 
$\tilde{x}_{2}=-A_{22}^{-1}\left(A_{21} \tilde{x}_{1}+B_{2} \tilde{u}\right)$.

Hence, substituting $\tilde{x}_{2}$ into (3.3), we can define the slow subsystem as

$\dot{x}_{s}=A_{s} x_{s}+B_{s} u_{s}$

where $A_{s}=A_{11}-A_{12} A_{22}^{-1} A_{21}$, and $B_{s}=B_{1}-A_{12} A_{22}^{-1} B_{2}$. Thus $\tilde{x}_{1}=x_{s}, \tilde{u}=u_{s}$ and $\tilde{x}_{2}$ are the slow parts of the corresponding variables in (3.1).

In order to derive the fast subsystem, Kokotovic [31] assumes that the slow variables are constant during fast transients; i.e. $\dot{\tilde{x}}_{2}=0$ and $\tilde{x}_{I}=x_{s}=$ constant.

Thus from equation (3.5) we can have,

$A_{22} \tilde{\boldsymbol{x}}_{2}=-A_{21} \tilde{\boldsymbol{x}}_{1}-B_{2} \tilde{\boldsymbol{u}}$

Equation (3.7) can be rewritten as,

$$
A_{2 I} \tilde{x}_{1}=-A_{22} \tilde{x}_{2}-B_{2} \tilde{u}
$$

Substituting the value of $A_{21} \tilde{x}_{1}$ given by equation (3.8) into equation (3.2) gives,

$$
\varepsilon \dot{x}_{2}(t)=-A_{22} \tilde{x}_{2}-B_{2} \tilde{u}+A_{22} x_{2}+B_{2} u
$$

Since Kokotovic assumes that $\dot{\tilde{x}}_{2}=0$, then we can rewrite equation (3.9) to be,

$$
\varepsilon\left(\dot{x}_{2}-\dot{\tilde{x}}_{2}\right)=A_{22}\left(x_{2}-\tilde{x}_{2}\right)+B_{2}(u-\tilde{u})
$$

Letting $x_{f}=x_{2}-\tilde{x}_{2}$ and $u_{f}=u-u_{s}$, the fast subsystem for the system given by equation (3.1) and (3.2) can be defined as,

$$
\varepsilon \dot{x}_{f}=A_{22} x_{f}+B_{2} u_{f}
$$




\subsection{Block diagonalization}

The quasi steady state is a good method for decoupling the full order system for sufficiently small $\varepsilon$. However, for real systems the perturbation parameter $\varepsilon$ is not zero. As a result, when using the quasi steady state method the eigenvalues of the slow and fast subsystems are no longer in the same position as the eigenvalues of the full order system[4]. To overcome this problem we employ the diagonalization method [30]. Consider a general two-time scale stable system possessing two widely separated groups of eigenvalues, as

$\dot{x}_{1}=A_{11} \mathbf{x}_{1}+A_{12} \mathbf{x}_{2}+B_{1} \mathbf{u}$

$\dot{x}_{2}=A_{21} \mathbf{x}_{1}+A_{22} \mathbf{x}_{2}+B_{2} \mathbf{u}$

where $\mathbf{x}_{1} \in R^{m}$ and $\mathbf{x}_{2} \in R^{n}$ are the state vectors and $\mathbf{u} \in R^{r}$ is the control vector. The matrices $A_{i j}$ and $B_{i}$ are of appropriate dimensions. We note that if there is a small parameter $\varepsilon$ associated with $\dot{x}_{2}$ this puts (3.12) and (3.13) in singularly perturbed form. We assume the " $m$ " eigenvalues of the system (3.12) and (3.13) are small (or close to the origin) and the remaining " $n$ " eigenvalues are large (or far from the origin), giving slow and fast responses, respectively. In other words, the system (3.12) and (3.13) has " $m$ " dominant modes and " $n$ " non-dominant modes. We arrange the eigenspectrum, $e(A)$, of the system in increasing order of absolute values as [31]

$e(A)=\left\{\lambda_{s 1}, \ldots, \lambda_{s m}, \lambda_{f 1}, \ldots ., \lambda_{f n}\right\}$

where $0 \leq\left|\lambda_{s 1}\right| \leq\left|\lambda_{s 2}\right| \leq \ldots . \leq\left|\lambda_{s m}\right|<\left|\lambda_{f 1}\right| \leq\left|\lambda_{f 2}\right| \leq \ldots . \leq\left|\lambda_{f n}\right|$, and $A=\left[\begin{array}{cc}A_{11} & A_{12} \\ A_{21} & A_{22}\end{array}\right]$. 
The system (3.12) and (3.13) is said to possess a two time scale property, if the largest absolute eigenvalue of the slow eigenspectrum $e\left(A_{s}\right)$,

$e\left(A_{s}\right)=\left\{\lambda_{s l}, \ldots . ., \lambda_{s m}\right\}$

is much smaller than the smallest absolute eigenvalues of the fast eigenspectrum $e\left(A_{f}\right)$

$e\left(A_{f}\right)=\left\{\lambda_{f l}, \ldots ., \lambda_{f m}\right\}$

That is [40]

$\varepsilon=\frac{\left|\lambda_{s m}\right|}{\left|\lambda_{f I}\right|} \ll 1$

It should be noted that $\varepsilon$ is a measure of the separation of the two time scales. It should also be noted that $\varepsilon$ represents an intrinsic property of the system described by equations (3.12), (3.13) and $\varepsilon$ does not have to appear explicitly in the system. The basic idea of using a two-time scale analysis in obtaining low order models is to decouple the slow and fast modes. This is achieved by using a two stage linear transformation [30][40]. The first stage is to use the transformation,

$\mathbf{x}_{f}=\mathbf{x}_{2}+L \mathbf{x}_{1}$

The goal is to transform the system described by equations (3.12) and (3.13) to upper block triangular form. Then the system described by equations (3.12) and (3.13) transforms into

$\left[\begin{array}{c}\dot{\mathbf{x}}_{1} \\ \dot{\mathbf{x}}_{f}\end{array}\right]=\left[\begin{array}{cc}A_{11}-A_{12} L & A_{12} \\ R(L) & A_{22}+L A_{12}\end{array}\right]\left[\begin{array}{l}\mathbf{x}_{1} \\ \mathbf{x}_{f}\end{array}\right]+\left[\begin{array}{c}B_{1} \\ B_{2}+L B_{1}\end{array}\right] \mathbf{u}$

The requirement now is that the matrix $R(L)$ be zero. This means that the $n \times m$ matrix $L$ must satisfy the algebraic equation,

$R(L)=L A_{11}+A_{21}-L A_{12} L-A_{22} L=0$ 
The selection of $L$ is discussed in the next subsection. Then the system described by equations (3.12) and (3.13) transforms into

$\left[\begin{array}{l}\dot{\mathbf{x}}_{1} \\ \dot{\mathbf{x}}_{f}\end{array}\right]=\left[\begin{array}{cc}A_{s} & A_{I 2} \\ 0 & A_{f}\end{array}\right]\left[\begin{array}{l}\mathbf{x}_{I} \\ \mathbf{x}_{f}\end{array}\right]+\left[\begin{array}{l}B_{I} \\ B_{f}\end{array}\right] \mathbf{u}$

where $A_{s}=A_{11}-A_{12} L, \quad A_{f}=A_{22}+L A_{12}, \quad B_{f}=B_{2}+L B_{l}$.

The second stage is to apply the transformation

$\mathbf{x}_{s}=\dot{\mathbf{x}}_{l}-M \mathbf{x}_{f}$

to (3.21) and choose the $(m \times n)$ matrix $M$ such that

$A_{s} M-M A_{f}+A_{12}=0$.

Again, the selection of $M$ is discussed in the next subsection. Consequently, (3.21) transforms into the following

$\left[\begin{array}{l}\dot{\mathbf{x}}_{s} \\ \dot{\mathbf{x}}_{f}\end{array}\right]=\left[\begin{array}{cc}A_{s} & 0 \\ 0 & A_{f}\end{array}\right]\left[\begin{array}{l}\mathbf{x}_{s} \\ \mathbf{x}_{f}\end{array}\right]+\left[\begin{array}{l}B_{s} \\ B_{f}\end{array}\right] \mathbf{u}$

where $B_{s}=B_{1}-M L B_{1}-M B_{2}$.

The system in equation (3.25) has the desired decoupled form where the slow and fast variables, $\mathbf{x}_{s}$ and $\mathbf{x}_{f}$, can be solved independently of each other. Note that the transformation (3.18) and (3.23) related the slow and fast variables, $\mathbf{x}_{s}$ and $\mathbf{x}_{f}$ with the original variables, $x_{1}$ and $x_{2}$ as

$\left[\begin{array}{l}\mathbf{x}_{s} \\ \mathbf{x}_{f}\end{array}\right]=\left[\begin{array}{cc}I_{I}-M L & -M \\ L & I_{2}\end{array}\right]\left[\begin{array}{l}\mathbf{x}_{I} \\ \mathbf{x}_{2}\end{array}\right]$

where $I_{1}$ and $I_{2}$ are identity matrices with the appropriate dimensions. This interestingly has the inverse transformation, 
$\left[\begin{array}{l}x_{1} \\ x_{2}\end{array}\right]=\left[\begin{array}{cc}L_{1} & M \\ -L & I_{2}-L M\end{array}\right]\left[\begin{array}{l}x_{s} \\ x_{f}\end{array}\right]$,

\subsubsection{Numerical aspects}

Consider the numerical solution of the nonlinear equations (3.20) and (3.24) to obtain the values of the matrices $L$ and $M$ using an fixed point iteration procedure [40][41] from (3.20)

$$
\begin{aligned}
& L_{i+1}=A_{22}^{-1}\left(A_{21}+L_{i} A_{11}-L_{i} A_{12} L_{i}\right) \\
& L_{0}=A_{22}^{-1} A_{21}
\end{aligned}
$$

where $A_{22}$ is assumed to be nonsingular.

If we let

$$
L=L_{0}+D
$$

then $D$ is the solution of

$$
D A_{0}-\left(A_{22}+L_{0} A_{12}\right) D-D A_{12} D+L_{0} A_{0}=0
$$

where

$$
A_{0}=A_{11}-A_{12} L_{0}
$$

It has been shown that if the inequality [40]

$$
\left\|A_{22}^{-1}\right\| \ll \frac{1}{3}\left(\left\|A_{0}\right\|+\left\|A_{12}\right\|\left\|L_{0}\right\|\right)^{-1}
$$

is satisfied, the unique real roots of (3.31) exist, and satisfy

$0 \ll\|D\| \ll \frac{2\left\|A_{0}\right\|\left\|L_{0}\right\|}{\left(\left\|A_{0}\right\|+\left\|A_{12}\right\|\left\|L_{0}\right\|\right)}$.

Similarly, the iterative procedure to solve for $M$ from (3.24) is [40], 
$M_{i+1}=\left[\left(A_{11}-A_{12} L\right) M_{i}-M_{i} L A_{12}\right] A_{22}^{-1}+A_{12} A_{22}^{-1}$

with $M_{0}=A_{12} A_{22}^{-1}$.

The sufficient condition for iterative algorithms given by equation (3.28) and equation

(3.35) to converge is given by [30][40],

$\left\|A_{22}^{-1}\right\|<<\frac{1}{3}\left[\left\|A_{o}\right\|+\left\|A_{12}\right\|\left\|L_{0}\right\|\right]^{-1}$

This condition will be used in section 3.6 to check the possibility of using the diagonalization method.

\subsection{Linear Feedback Control}

\subsubsection{Introduction}

In the singular perturbation method, the full order model is separated into two decoupled systems. One of the obvious advantages for using the singularly perturbed method is that we can design two separate controllers one for each subsystem. These two controllers are used to control the full order model given by equations (3.1) and (3.2).

Assuming that the matrix pairs $A_{s}, B_{s}$ and $A_{22}, B_{2}$ in equations (3.6) and (3.11) are controllable pairs, then one can use full order feedback and pole placement techniques to arbitrarily place the closed loop poles based on the desired performance specified by the designer.

\subsubsection{Composite state feedback control}

The requirement is to design a state feedback controller for the singularly perturbed linear time invariant system model given by equations (3.1)and (3.2) that have the form, 
$\dot{x}_{1}(t)=A_{l 1} x_{l}(t)+A_{l 2} x_{2}(t)+B_{l} u(t)$

$\varepsilon \dot{x}_{2}(t)=A_{21} x_{1}(t)+A_{22} x_{2}(t)+B_{2} u(t)$

Preliminary to any separation of slow and fast designs, the system represented by equations (3.37) and (3.38) is approximately decomposed into a slow system model with " $n$ " small eigenvalues and a fast system model with " $m$ " large eigenvalues, as mentioned in section (3.2). The $\mathrm{n}^{\text {th }}$ order slow system is given as,

$\dot{x}_{s}=A_{s} x_{s}+B_{s} u_{s}$

$\tilde{\boldsymbol{x}}_{2}=-A_{22}^{-1}\left(A_{21} \boldsymbol{x}_{s}+B_{2} \boldsymbol{u}_{s}\right)$

where $A_{s}=A_{11}-A_{12} A_{22}^{-1} A_{21}, B_{s}=B_{1}-A_{12} A_{22}^{-1} B_{2}$ and the vectors $\boldsymbol{x}_{s}, \tilde{x}_{2}$ and $\boldsymbol{u}_{s}$ are the slow parts of the corresponding variables $x_{1}, x_{2}$ and $u$ in the original system given by equation (3.37) and (3.38). Also the $\mathrm{m}^{\text {th }}$-order fast subsystem is defined as,

$\varepsilon \dot{x_{f}}=A_{22} x_{f}+B_{2} u_{f}$

where $x_{f}=x_{2}-\tilde{x}_{2}$ and $u_{f}=u-u_{s}$ denote the fast parts of the corresponding variables in system (3.37) and (3.38).

It is appropriate to consider the following decomposition [30] of feedback controls $u_{s}=G_{0} x_{s}$ and $u_{f}=G_{2} x_{f}$ that are separately designed for the slow and fast subsystems given by equation (3.39) and (3.41) respectively. A composite control for the full order system given by equation (3.37) and (3.38) can then be taken as

$u_{s}+u_{f}=G_{0} x_{s}+G_{2} x_{f}$

However, a realizable composite control requires that the system states $x_{s}$ and $x_{f}$ be expressed in terms of the actual system states $x_{1}$ and $x_{2}$. This can be achieved by 
replacing $x_{s}$ by $x_{1}$ and $x_{f}$ by $x_{2}-\tilde{x}_{2}$, so that the composite control given by equation (3.42), takes the realizable feedback form,

$$
\begin{gathered}
u=G_{0} x_{1}+G_{2}\left[x_{2}+A_{22}^{-1}\left(A_{2 I} x_{1}+B_{2} G_{0} x_{1}\right)\right] \\
=G_{1} x_{1}+G_{2} x_{2}
\end{gathered}
$$

where $G_{1}=\left(I+G_{2} A_{22}^{-1} B_{2}\right) G_{0}+G_{2} A_{22}^{-1} A_{2 I}$

The above design procedure is a decomposed one in that the gain matrices $G_{0}$ and $G_{2}$ are separately designed according to slow and fast mode performance specifications, resulting in the physical realizable composite control law given by equation (3.44).

The feedback control design for singularly perturbed systems can also be based on the block diagonalization method given in section 3.3 [40]. The idea is to first obtain the reduced order system using the diagonalization method for the system given by equations (3.37) and (3.38). The reduced order system will have the form,

$$
\left[\begin{array}{c}
\dot{\mathbf{x}}_{s} \\
\dot{\mathbf{x}}_{f}
\end{array}\right]=\left[\begin{array}{cc}
A_{s} & 0 \\
0 & A_{f}
\end{array}\right]\left[\begin{array}{l}
\mathbf{x}_{s} \\
\mathbf{x}_{f}
\end{array}\right]+\left[\begin{array}{l}
B_{s} \\
B_{f}
\end{array}\right] \mathbf{u}
$$

where $A_{s}=A_{11}-A_{12} L, \quad A_{f}=A_{22}+L A_{12}, \quad B_{f}=B_{2}+L B_{1}$ and $B_{s}=B_{1}-M L B_{1}-M B_{2}$ Two separate controllers $F_{1}$ and $F_{2}$ are designed based on the slow and fast subsystems given in equation (3.46) such that $A_{s}+B_{s} F_{l}$ satisfies the slow subsystem desired performance and $A_{f}+B_{f} F_{2}$ satisfies the fast subsystem desired performance. The control law that will control the full order system has the form

$$
\boldsymbol{u}=F \boldsymbol{y}
$$

where $y^{T}=\left(x_{1}^{T}, x_{2}^{T}\right)$ and $F$ is given by [40], 


$$
F=\left[F_{2} L+\left(F_{l}-F_{2} L\right)(I-M L)-F_{1} M+F_{2}(I+L M)\right]
$$

The asymptotic stability of the composite control given by equation (3.44) or equation (3.47) when applied to system (3.37) and (3.38) is studied in many articles and can be seen in books by Khalil [28] and Naidu [40].

\subsection{Stability analysis}

\subsubsection{Introduction}

Many methods for stability analysis for closed loop singularly perturbed systems can be found. A Lyapunov function based approach is one of the earliest introduced by Kokotovic [30]. In this approach, which is discussed in detail in this section, the computation of the upper bound $\varepsilon^{*}$ is based on the solution of two algebraic Lyapunov equations.

Other methods can be found to determine the maximum values of $\varepsilon$ that ensure that the closed loop system is stable which are:

1- Frequency- domain transfer function based techniques which use Nyquist stability criteria to define the maximum value of the perturbed parameter [5][18].

2- State space model based techniques which use robust control stability analysis to find the stable region for a singularly perturbed system [7].

Both of these techniques can provide improved stability bounds compared to the Lyapunov function based approach as shown in Cao [5] and Chen [7]. However these techniques use linear systems analysis. Sliding mode controllers introduce a nonlinearity to the closed loop system. Extension of the above mentioned methods to nonlinear systems 
make the analysis very complicated. Consequently, we will use a Lyapunov approach to discuss the stability analysis of sliding mode controllers when used with singularly perturbed systems.

\subsubsection{Lyapunov stability analysis for singularly perturbed system}

Consider a nonlinear singularly perturbed system [30]

$$
\begin{aligned}
& \dot{x}=f(x, z) \\
& \varepsilon \dot{z}=g(x, z)
\end{aligned}
$$

where $x \in R^{n}$ and $z \in R^{m}$. It is assumed that equation (3.49) and equation (3.50) have a unique solution for given initial conditions. Assume also that the system given by equations (3.49) and (3.50) have equilibrium points at the origin. The stability of the origin can be investigated using the reduced slow system

$$
\dot{x}=f\left(x, z_{s}\right)
$$

where $z_{s}=h(x)$ is an isolated root of $0=g(x, z)$ and represents the steady state solution of the fast states. In the quasi steady state method, we assume that the slow variables are constant during the fast transient, that is $\dot{z}=0$ and $x=x_{s}=$ constant. Then the fast variations are the deviations of $z$ from its quasi steady state. Fast states can be given by,

$$
x_{f}=z-z_{s}=z-h(x)
$$

The main goal now is to show that for sufficiently small $\varepsilon$, the origin is a uniformly asymptotically stable equilibrium of the singularly perturbed system given by equations (3.49) and (3.50), given that $x=0$ is a uniformly asymptotically stable equilibrium of the reduced system given by equation (3.51) and $z_{s}=h(x)$ is an asymptotically stable 
equlibrium of the fast subsystem. To prove this we make the following assumptions $[30][40]$.

\section{Assumption 1}

The origin $(x=0, z=0)$ is the unique equilibrium of the system given by equation (3.49) and (3.50) that is, $0=f(0,0)$ and $0=g(0,0)$. The equation $0=g(x, z)$ has a unique root $z_{s}$.

\section{Assumption 2 (Reduced system)}

There exist a Lyapunov function $v(x)$ satisfying:

1- $\quad v(x)$ is positive definite and decrescent

$$
\text { 2- } \frac{\partial v}{\partial x} f\left(x, z_{s}\right) \leq-\alpha_{1} \psi^{2}(x) \quad\left(\alpha_{1}>0\right)
$$

where $\psi(\cdot)$ is a continuous scalar function of $x$ which vanishes only at $x=0$.

\section{Assumption 3}

There exists a bounded Lyapunov function $w(x, z)$ satisfying

$$
\frac{\partial w}{\partial z} g(x, z) \leq-\alpha_{2} \phi^{2}\left(z-z_{s}\right) \quad \alpha_{2}>0
$$

where $\phi$ is a continuous function of an $R^{m}$ vector $\left(z-z_{s}\right)$ that vanishes only at $\left(z-z_{s}\right)=0$

\section{Assumption 4 (Interconnection conditions) [30][40]}

Functions $v$ and $w$ satisfy the following inequalities

$$
1-\quad \frac{\partial v}{\partial x}\left(f(x, z)-f\left(x, z_{s}\right) \leq \beta_{1} \psi(x) \phi\left(z-z_{s}\right)\right.
$$


$2-\quad \frac{\partial w}{\partial x} f(x, z) \leq \gamma \phi^{2}\left(z-z_{s}\right)+\beta_{2} \psi(x) \phi\left(z-z_{s}\right)$

for simplicity the constants $\beta_{1}, \beta_{2}$ and $\gamma$ are assumed to be nonnegative.

The condition given by equation (3.54) determines the allowable growth of $f(x, z)$ in $z$. Usually verifying the condition given by equation (3.54) reduces to verifying the inequality,

$\left\|f(x, z)-f\left(x, z_{s}\right)\right\| \leq \phi\left(z-z_{s}\right)$

which means that the rate of growth of $f(x, z)$ with respect to $z$ cannot be faster than the rate of growth of $\phi($.$) . Similarly the condition given by equation (3.55) determines the$ allowable growth of $g(x, z)$ in $x$ given that $w(x, z)$ can be seen as Lyapunov function of $g(x, z)$.

Given the Lyapunov functions of the reduced subsystems, $v$ and $w$, based on Kokotovic's [30] approach we consider a Lyapunov function $J(x, z)$ given by the weighted sum of $v$ and $w$ that takes the form,

$$
J(x, z)=(1-d) v(x)+d w(x, z) \quad(0<d<1)
$$

From the properties of $v$ and $w$ given by equation (3.52), (3.53), (3.54) and (3.55) it follows that $J(x, z)$ is positive definite and decreasing. Computing $\dot{J}$ with respect to equations (3.49) and (3.50) and using equations from (3.52) to (3.55) we obtain,

$$
\begin{aligned}
j \leq & -\left[\begin{array}{c}
\psi(x) \\
\phi\left(z-z_{s}\right)
\end{array}\right]^{T} \times \\
& {\left[\begin{array}{cc}
(1-d) \alpha_{1} & -1 / 2(1-d) \beta_{1}-1 / 2 d \beta_{2} \\
-1 / 2(1-d) \beta_{1}-1 / 2 d \beta_{2} & d\left(\frac{\alpha_{2}}{\varepsilon}-\gamma\right)
\end{array}\right]\left[\begin{array}{c}
\psi(x) \\
\phi\left(z-z_{s}\right)
\end{array}\right] }
\end{aligned}
$$

The quadratic form of the above equation is negative definite when, 


$$
d(1-d) \alpha_{1}\left(\frac{\alpha_{2}}{\varepsilon}-\gamma\right)>\frac{1}{4}\left[(1-d) \beta_{1}+d \beta_{2}\right]^{2}
$$

that can be rearranged to give,

$$
\frac{1}{\varepsilon}>\frac{1}{\alpha_{1} \alpha_{2}}\left[\alpha_{1} \gamma+\frac{1}{4(1-d) d}\left((1-d) \beta_{1}+d \beta_{2}\right)^{2}\right]
$$

From the above equation we notice that for any choice of $d(d \in(0,1))$ and for all $\varepsilon$ satisfying equation (3.59), $J$ is a Lyapunov function for the system given by equations (3.49) and (3.50). Equation (3.59) can be rewritten as

$$
\varepsilon<\frac{\alpha_{1} \alpha_{2}}{\alpha_{1} \gamma+\frac{1}{4(1-d) d}\left[(1-d) \beta_{1}+d \beta_{2}\right]^{2}}=\varepsilon_{d}
$$

Kokotovic [30] shows that the maximum value of $\varepsilon_{d}=\varepsilon^{*}$ and occurs at $d^{*}=\frac{\beta_{1}}{\beta_{1}+\beta_{2}}$ and is given by,

$$
\varepsilon^{*}=\frac{\alpha_{1} \alpha_{2}}{\alpha_{1} \gamma+\beta_{1} \beta_{2}}
$$

\section{Theorem (3.1) [30][40]}

Given the assumptions (1), (2), (3) and (4) then the origin is an asymptotically stable equilibrium of the singularly perturbed system given by equation (3.49) and equation (3.50) for all $\varepsilon \in\left(0, \varepsilon^{*}\right)$, where $\varepsilon^{*}$ is given by (3.61)

The proof for the above theorem can be found in detail in [30] chapter 7 and in appendix A. We have to stress that this is a sufficient condition but not a necessary condition for stability. 


\subsection{Example of the Flexible Shaft}

The single link flexible joint manipulator directly actuated by an electric motor is taken as an example to demonstrate the method of linear feedback control for a singularly perturbed system.

For the sake of simplicity, some important phenomena like joint flexibility, actuator dynamics and friction, have been neglected in the simplest control algorithms developed for robotic applications. However, experimental results have shown that the joint elasticity should be taken into account in the modeling of some robotic manipulators.

For modeling purposes, the manipulator consists of an actuator whose rotor axis, with inertia $\left(J_{r}\right)$ and viscous damping $\left(\zeta_{l}\right)$, is directly connected to an axis with inertia $\left(J_{m}\right)$ and viscous damping $\left(\varsigma_{2}\right)$. The elasticity in the joint is modeled as a torsional spring with known characteristics (a linear spring with stiffness $K$ is considered), as shown in Figure 3.1.

The motor shaft angular velocity $\left(\omega_{1}\right)$, and link angular velocity $\left(\omega_{2}\right)$, are taken as the generalized coordinates. For this single link robot, the dynamic equations are

$J_{r} \dot{\omega}_{I}+\zeta_{I} \omega_{I}+K \bar{\theta}=T$

$J_{m} \dot{\omega}_{2}+\zeta_{2} \omega_{2}-K \bar{\theta}=0$

$\bar{\theta}=q_{1}-q_{2}$

where $T$ is the torque generated by the actuator and $q_{1}, q_{2}$ are the angles of motor shaft and link respectively. Let $T=u$ for the modeling purpose.

The above model can be put in singularly perturbed form by defining 
$z=\omega_{1}$ and $\varepsilon=J_{r}$.

The system equations have the form

$$
\left[\begin{array}{c}
\varepsilon \dot{\omega}_{1} \\
\dot{\omega}_{2} \\
\dot{\bar{\theta}}
\end{array}\right]=\left[\begin{array}{ccc}
-\varsigma_{1} & 0 & -K \\
0 & -\frac{\varsigma_{2}}{J_{m}} & \frac{K}{J_{m}} \\
1 & -1 & 0
\end{array}\right]\left[\begin{array}{c}
\omega_{1} \\
\omega_{2} \\
\bar{\theta}
\end{array}\right]+\left[\begin{array}{l}
1 \\
0 \\
0
\end{array}\right] u
$$

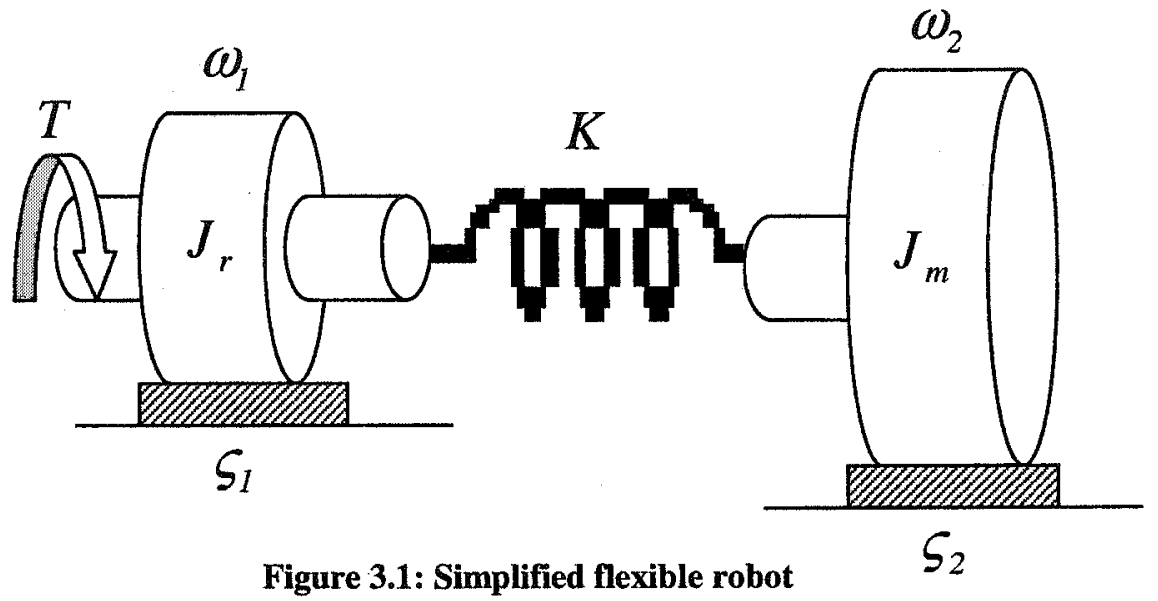

The above state equation can be put in state space singularly perturbed form as:

$$
\begin{aligned}
& \dot{\boldsymbol{x}}=\left[\begin{array}{c}
\dot{\omega}_{2} \\
\dot{\bar{\theta}}
\end{array}\right]=\left[\begin{array}{cc}
-\frac{\varsigma_{2}}{J_{m}} & \frac{K}{J_{m}} \\
-1 & 0
\end{array}\right]\left[\begin{array}{c}
\omega_{2} \\
\bar{\theta}
\end{array}\right]+\left[\begin{array}{l}
0 \\
1
\end{array}\right] \omega_{1}+\left[\begin{array}{l}
0 \\
0
\end{array}\right] u \\
& \varepsilon \dot{z}=\varepsilon \dot{\omega}_{1}=\left[\begin{array}{ll}
0 & -K
\end{array}\right]\left[\begin{array}{c}
\omega_{2} \\
\bar{\theta}
\end{array}\right]+\left[-\varsigma_{1}\right] \omega_{1}+[l] u .
\end{aligned}
$$

Assume the following parameters,

$J_{r}=0.04 \mathrm{Kg} \cdot \mathrm{m}^{2}, \quad J_{m}=1 \mathrm{Kg} \cdot \mathrm{m}^{2}$,

$\varsigma_{1}=0.15 \mathrm{~N} /(\mathrm{rad} / \mathrm{s}), \quad \varsigma_{2}=0.5 \mathrm{~N} /(\mathrm{rad} / \mathrm{s}), K=25 \mathrm{~N} . \mathrm{m} / \mathrm{rad}$

Then the system equation becomes

$$
\dot{\boldsymbol{x}}=\left[\begin{array}{c}
\dot{\omega}_{2} \\
\dot{\bar{\theta}}
\end{array}\right]=\left[\begin{array}{cc}
-0.5 & 25 \\
-1 & 0
\end{array}\right]\left[\begin{array}{c}
\omega_{2} \\
\bar{\theta}
\end{array}\right]+\left[\begin{array}{l}
0 \\
1
\end{array}\right] \omega_{1}+\left[\begin{array}{l}
0 \\
0
\end{array}\right] u
$$




$$
\varepsilon \dot{z}=\left[\begin{array}{ll}
0 & -25
\end{array}\right]\left[\begin{array}{c}
\omega_{2} \\
\bar{\theta}
\end{array}\right]+[-0.15] \omega_{1}+[1] u
$$

Two methods to obtain the reduced order subsystem can be used as described in the previous sections. For this example, the diagonalization method may not converge since $A_{22}$ does not satisfy the sufficient convergence condition given by equation (3.36). Using the quasi steady state method, the reduced slow subsystem can be obtained by letting $\varepsilon=0$. From Kokotovic [31] we get,

$\dot{\boldsymbol{x}}_{s}=A_{s} x_{s}+B_{s} u_{s}$

where $A_{s}=A_{11}-A_{12} A_{22}^{-1} A_{21}=\left[\begin{array}{cc}-0.5 & 25 \\ -1 & -166.67\end{array}\right]$

and $B_{s}=\left(B_{1}-A_{12} A_{22}^{-1} B_{2}\right)=\left[\begin{array}{c}0 \\ 6.667\end{array}\right]$

The fast subsystem is given by equation (3.41). For our example this takes the form $\varepsilon \dot{x}_{f}=[-0.15] x_{f}+[I] u_{f}$

while the slow subsystem can be rewritten as, $\dot{x}_{s}=\left[\begin{array}{cc}-0.5 & 25 \\ -1 & -166.67\end{array}\right] x_{s}+\left[\begin{array}{c}0 \\ 6.667\end{array}\right] u_{s}$

The desired two slow poles are taken to be " -0.2 " and " -0.1 ", while the fast pole will be taken to be "-1". Using the pole placement design techniques described in many linear control books, we can easily determine $G_{0}$ and $G_{2}$ based on the slow and fast subsystems respectively. The desired characteristic equation for the slow subsystem is given by, $S^{2}+0.3 S+0.02=0$

Checking the controllability by calculating the rank 
$\operatorname{rank}(M)=\operatorname{rank}\left[\begin{array}{ll}B_{s} & A_{s} B_{s}\end{array}\right]=\operatorname{rank}\left[\begin{array}{cc}0 & 0.1667 \\ 0.0067 & -1.111\end{array}\right]=2$,

which means that the system is controllable. The feed back gain $G_{0}$ can be calculated from the following equation,

$\operatorname{det}\left|S I-A_{s}+B_{s} G_{0}\right|=S^{2}+0.3 S+0.02$

thus the feedback gain for the slow subsystem is $G_{0}=\left[\begin{array}{ll}-0.1493 & -25\end{array}\right]$. The feedback gain for the fast subsystem can be calculated from $\operatorname{det}\left|S I-A_{22}+B_{2} G_{2}\right|=S+1$, thus $G_{2}=[0.85]$. Based on equation (3.45) the value of feedback gain $G_{I}$ is $G_{1}=\left[\begin{array}{ll}0.6966 & 258.47\end{array}\right]$. The time response of the closed loop system for the full order system, given by equation (3.65), is shown in Figure 3.2. The responses in these figures are initial condition responses, which are, $x_{f}(0)=0$ and $x_{s}(0)=\left[\begin{array}{c}0.2 \\ 0\end{array}\right]$

This example demonstrates the state feedback control method that is used to control the full order singularly perturbed model. In this case we see that the controller can handle both the fast and slow subsystems and this means that fast subsystem is not ignored but has been taken into account. 


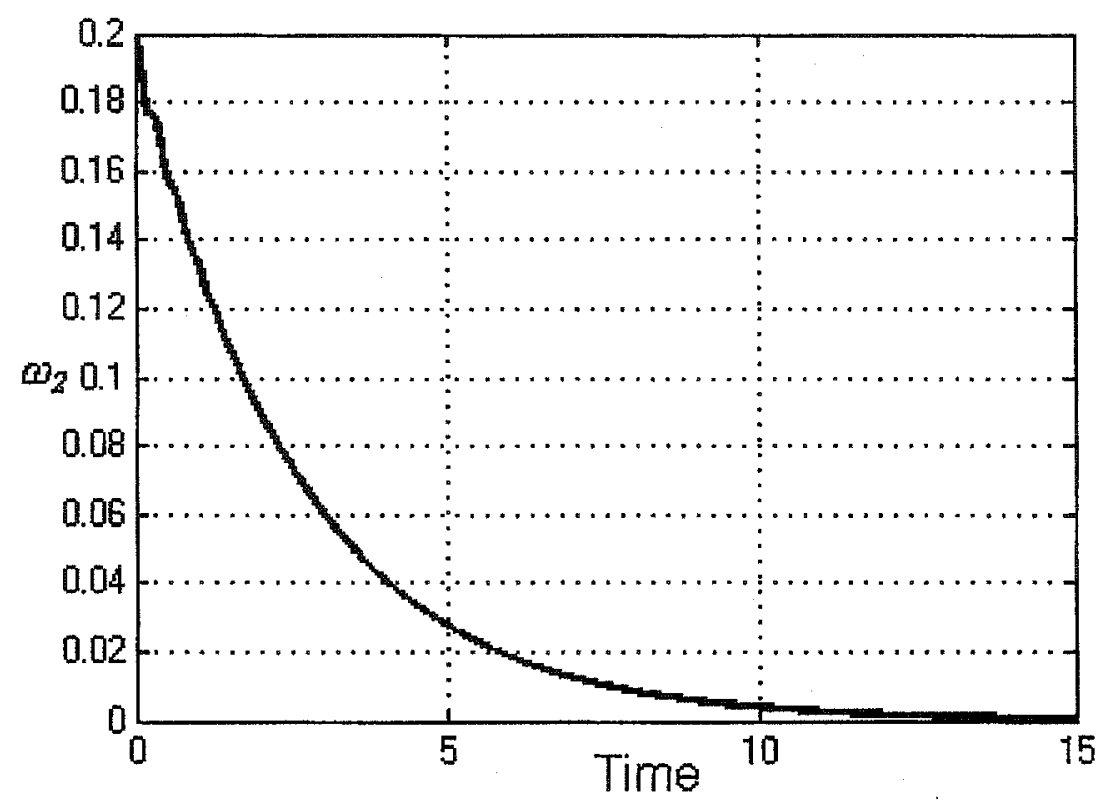

Figure 3.2.a: Closed loop time response of manipulator angular velocity

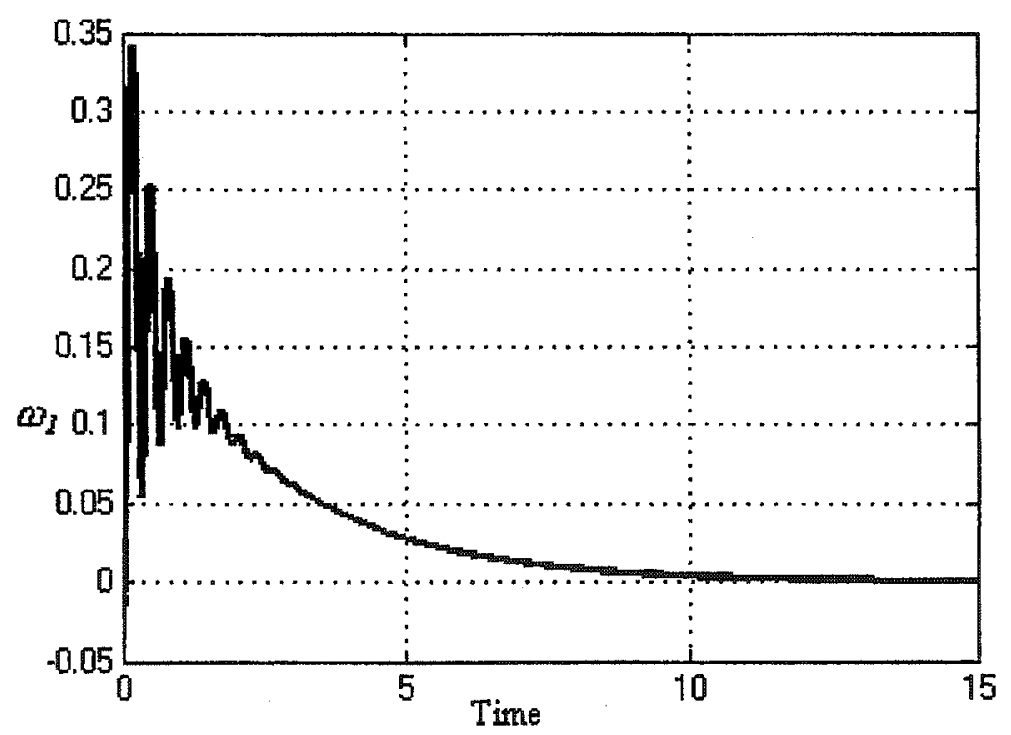

Figure 3.2.b: Closed loop time response of rotor angular velocity

In this chapter we discussed the singular perturbation method. Different decoupling algorithms have been explained. The decoupling methods are used to obtain reduced order systems from the full order singularly perturbed system. 
Stability analysis for nonlinear singularly perturbed model has been reviewed. The interval for perturbation parameter $\varepsilon \in\left(0, \varepsilon^{*}\right)$ for the full order system is obtained where the closed loop system is asymptotically stable. This will be a very useful approach to prove stability of the single sliding mode controller for the full order singularly perturbed system. 


\section{Chapter 4 Review of Sliding Mode Control}

\section{For A Singularly Perturbed System}

This chapter briefly reviews the techniques of sliding mode control for singularly perturbed systems that have been reported in the literature. The techniques reported discuss the use of two independent sliding mode controllers. These methods require the measurement of the states typically associated with the high frequency dynamic modes which are usually unavailable or not measured. This chapter serves as a point of reference for the ideas and contributions presented in later chapters.

Slotine [50] introduced a two-time scale sliding mode controller where he used singular perturbation theory to design two sliding mode controllers, one for the slow subsystem and one for the fast subsystem based on his tracking control approach [49]. Heck [22] similarly addressed the use of two sliding mode controllers to control singularly perturbed systems; however she uses a different control strategy to build the control law. Li [37] investigated the results from Heck's article [22] and proposed a condition to ensure that the controller satisfies the sliding condition for the full order system.

Other papers [11][20][60] discuss Heck's approach and define another method for stability and robustness analysis. In this chapter we investigate the use of sliding mode control for singularly perturbed systems. 


\subsection{Model Reduction Using Singular Perturbation}

Consider the linear time- invariant system represented by

$$
\begin{array}{ll}
\dot{x}=A_{11} x+A_{12} z+B_{1} u & x\left(t_{0}\right)=x_{0} \\
\varepsilon \dot{z}=A_{21} x+A_{22} z+B_{2} u & z\left(t_{0}\right)=z_{0}
\end{array}
$$

where $x \in R^{n}, z \in R^{m}$ and $u \in R^{r}$. The term $\varepsilon$ is a small positive parameter and $A_{22}$ is restricted to be stable [28]. This system is singularly perturbed due to the presence of $\varepsilon>0$. This system can be separated into reduced order models. One system contains the fast dynamics and the other contains the slow dynamics as shown in the previous chapter. The slow dynamic model can be obtained by setting $\varepsilon=0$ in (4.2). The resulting reduced order system is given as follows:

$\dot{\boldsymbol{x}}_{s}=A_{11} \boldsymbol{x}_{s}+A_{12} z_{s}+B_{1} u_{s} \quad \boldsymbol{x}\left(t_{0}\right)=\boldsymbol{x}_{0}$

where $z_{s}=-A_{22}^{-1}\left(A_{21} x_{s}+B_{2} u_{s}\right)$.

In (4.3) $\boldsymbol{u}_{s}$ is the slow component of the control. If $\boldsymbol{u}_{s}$ depends only on $\boldsymbol{x}_{\boldsymbol{s}}$ the slow model can be expressed by

$\dot{x}_{s}=A_{s} x_{s}+B_{s} u_{s}$

$z_{s}=-A_{22}^{-1}\left(A_{21} x_{s}+B_{2} u_{s}\right)$

where $A_{s}=\left(A_{11}-A_{12} A_{22}^{-1} A_{21}\right)$ and $B_{s}=\left(B_{1}-A_{12} A_{22}^{-1} B_{2}\right)$

The quasi steady state solution defines a "slow manifold" in the full order state space. The fast dynamic model can be easily obtained as discussed in section (3.2.1) and it takes the same form as equation (3.11),

$$
\dot{\eta}=A_{22} \eta+B_{2} u_{f} \quad \eta(0)=z_{0}-z_{s 0}=z_{0}-h\left(x_{0}\right)
$$


where $\boldsymbol{u}_{f}=\boldsymbol{u}-\boldsymbol{u}_{s}, \boldsymbol{\eta}=z-z_{s}$ and $z_{s 0}$ is the initial condition for $z_{s}$. This shows that the fast state can be represented by the difference between the full order state $z$ and its steady state value $z_{s}$.

The idea of using sliding mode control for a singularly perturbed system that was investigated before is to design separate control laws for the fast dynamic model and for the slow dynamic model. The controls are then combined in a composite form for implementation on the full order model.

\subsection{Slow Sliding Mode Control Design}

The slow sliding mode controller is designed using the slow reduced order model in equation (4.4). The equivalent control method by Utkin [55] is used to design such a controller. The slow subsystem as described before has the form

$$
\dot{x}_{s}=A_{s} x_{s}+B_{s} u_{s}
$$

The equivalent control law for this system as described in section (2.2) equation (2.8) has the form

$$
u_{e s}=-\left[\left(\frac{\partial S_{s}}{\partial x_{s}} B_{s}\right)^{-1} \frac{\partial S_{s}}{\partial x_{s}} A_{s}\right] x_{s}
$$

Assume that $S_{s}$ is a linear switching surface with respect to $\mathrm{x}_{s}$ so that equation (4.8) can be rewritten as

$$
\boldsymbol{u}_{e s}=-\left[\left(C_{s} B_{s}\right)^{-1} C_{s} A_{s}\right] \boldsymbol{x}_{s}
$$

where $S_{s}=C_{s} \mathbf{x}_{s}$ and $C_{s}$ is a matrix of appropriate dimensions that defines the sliding surface properties. The substitution of $u_{e s}$ from equation (4.9) into equation (4.7) gives 
us the equivalent sliding mode equation, which describes the dynamics of the system while sliding

$$
\dot{\mathbf{x}}_{e q s}=\left[I-B_{s}\left(C_{s} B_{s}\right)^{-1} C_{s}\right] A_{s} \mathbf{x}_{e q s}=A_{e q} \mathbf{x}_{e q s},
$$

where $I$ is the identity matrix. The elements of $C_{s}$ are chosen so that the sliding mode dynamics for the slow subsystem are stable and have the desired performance based on equation (4.10) (as described in section (2.2)).

It is required also to design the second part of the control law which is the switching feedback gains that will drive the state trajectory to the sliding surface and maintain it there. This can be done by choosing the Lyapunov function for a single input single output (SISO) system as,

$$
V_{s}=\frac{1}{2} S_{s}^{2}
$$

For convergence to the sliding surface it is required that the derivative of $V_{s}(x)$ be negative

$$
\dot{V}=S_{s} \dot{S}<0
$$

From the analysis in chapter 2 section (2.2.2) the control law is given by,

$$
u_{s}=-\left[C_{s} B_{s}\right]^{-1} C_{s} A_{s} x_{s}-\left[C_{s} B_{s}\right]^{-1} \mu_{1} \operatorname{sgn}\left(S_{s}\right)
$$

where $\mu_{1}$ is an arbitrary positive constant. Equation (4.13) is the sum of two terms; one is the equivalent control term and the other is the switching control term.

\subsection{Fast Sliding Mode Control Design}

The fast sliding mode controller is designed for the fast-reduced order model given in (4.6) as 


$$
\dot{\eta}=A_{22} \eta+B_{2} u_{f} \quad \eta(0)=z_{0}-z_{s 0}
$$

The switching surface in this case takes the form

$$
S_{f}=C_{f} \boldsymbol{\eta}=0
$$

Similar to the slow subsystem the equivalent control for this system takes the form

$$
u_{e f}=-\left(C_{f} B_{2}\right)^{-1} C_{f} A_{22} \eta
$$

where $\boldsymbol{u}_{e f}$ is the equivalent control for the fast subsystem. Substituting $\boldsymbol{u}_{e f}$, obtained from (4.16) into (4.14) yields the sliding mode equation which describes the dynamics during sliding,

$$
\dot{\eta}=\left[I-B_{2}\left(C_{f} B_{2}\right)^{-1} C_{f}\right] A_{22} \boldsymbol{\eta}
$$

The elements of $C_{f}$ are chosen so that the sliding mode dynamics are stable and have the desired performance. This can be done based on either Utkin's approach [55] or Slotine's approach [48].

Given the Lyapunov function for the SISO fast subsystem as,

$$
V_{f}=\frac{1}{2} S_{f}^{2}
$$

thus the reaching condition specified in chapter (2) section (2.2.2) is satisfied if we have,

$$
V_{f}=S_{f} \dot{S}_{f}<0
$$

The control law for a fast subsystem includes both an equivalent control term and a discontinuous control term and is given by, $u_{f}=u_{e f}-\mu_{2} \operatorname{sgn}\left(S_{f}\right)$

Thus

$$
u_{f}=-\left[\left(C_{f} B_{2}\right)^{-1} C_{f} A_{22}\right] \eta-\left(C_{f} B_{2}\right)^{-1} \mu_{2} \operatorname{sgn}\left(S_{f}\right)
$$




\subsection{Composite Control}

The composite control approach employs two independent control components, which are the slow and fast control laws. The output of each control law is summed before being applied to the plant. The slow control component is responsible for the tracking of the commanded slow state trajectories, and is designed based on the slow reduced model. The fast control is used to control the fast system dynamics and is designed based on the fast-reduced model. In the actual implementation to the full order model given in (4.1) and (4.2), the control would be a composite of the slow and fast controls as follows:

$\boldsymbol{u}=\boldsymbol{u}_{s}\left(\boldsymbol{x}_{s}\right)+\boldsymbol{u}_{f}\left(z-z_{s}\right)$

where $z_{s}=h\left(x_{s}\right)$.

Heck [22] proposed two theorems to ensure the stability of the full order model when the composite reduced controller is applied to it.

Theorem (4.1) [22]

Suppose that the fast control is chosen so that the reaching condition given by equation (4.18) is satisfied for the fast model. Then there exist $\varepsilon^{*}>0$ such that the reaching condition given by equation (4.18) will be satisfied for the full order model for all $\varepsilon \in\left(0, \varepsilon^{*}\right)$

Theorem (4.2) [22]

Suppose that the slow control is chosen so that the reaching condition given by equation (4.12) is satisfied for the fast model. Then there exist $\varepsilon^{*}>0$ such that the reaching condition given by equation (4.12) will be satisfied for the full order model for all $\varepsilon \in\left(0, \varepsilon^{*}\right)$ 
Substituting the value of $z_{s}$ given by equations (4.5) into equation (4.20) gives us

$$
\boldsymbol{u}=\boldsymbol{u}_{s}+\boldsymbol{u}_{f}\left(z+A_{22}^{-1}\left(A_{21} \boldsymbol{x}_{s}+B_{2} \boldsymbol{u}_{s}\right)\right)
$$

Substituting the value of $u_{f}$ given by equation (4.19) into equation (4.21) leads us to $u=u_{s}+\left[-\left(C_{f} B_{2}\right)^{-1} C_{f} A_{22}\left[z+A_{22}^{-1}\left(A_{2 I} x_{s}+B_{2} u_{s}\right)\right]-\left(C_{f} B_{2}\right)^{-1} \mu_{2} \operatorname{sgn}\left(S_{f}\right)\right]$

However, a realizable composite control requires that the system states " $\boldsymbol{x}_{s}$ " and " $\boldsymbol{\eta}$ " be expressed in term of actual states " $x$ " and " $z$ "[30][51]. This can be done by replacing " $x_{s}$ " by " $x$ " and " $\boldsymbol{\eta}$ " by " $z-h(x)$ " thus the control law given by equation (4.21) can be rewritten to be,

$$
u=-\left(C_{f} B_{2}\right)^{-1} C_{f} A_{21} x-\left(C_{f} B_{2}\right)^{-1} C_{f} A_{22} z-\left(C_{f} B_{2}\right)^{-1} \mu_{2} \operatorname{sgn}\left(S_{f}\right)
$$

Thus the full order control law $\boldsymbol{u}$ can be represented with two terms by writing equation (4.22) to be

$$
u=-\left(C_{f} B_{2}\right)^{-1} C_{f}\left[\begin{array}{ll}
A_{21} & A_{22}
\end{array}\right]\left[\begin{array}{l}
x \\
z
\end{array}\right]-\left(C_{f} B_{2}\right)^{-1} \mu_{2} \operatorname{sgn}\left(S_{f}\right)
$$

where $\mu_{2}$ is arbitrary positive constant.

The first term in the right hand side is the equivalent control and the second term is the regulating control for the full order system, that is

$$
\boldsymbol{u}=\boldsymbol{u}_{e q}+\Delta u
$$

where $u_{e q}=-\left(C_{f} B_{2}\right)^{-1} C_{f}\left[\begin{array}{ll}A_{2 I} & A_{22}\end{array}\right]\left[\begin{array}{l}x \\ z\end{array}\right]$ and $\Delta u=-\left(C_{f} B_{2}\right)^{-1} \mu_{2} \operatorname{sgn}\left(S_{f}\right)$.

Applying $\boldsymbol{u}_{e q}$ to the original perturbed system given by (4.1) and (4.2) gives 


$$
\left[\begin{array}{c}
\dot{x} \\
\dot{z}
\end{array}\right]=\left[\begin{array}{cc}
A_{11}-B_{1}\left(C_{f} B_{2}\right)^{-1} C_{f} A_{21} & A_{12}-B_{1}\left(C_{f} B_{2}\right)^{-1} C_{f} A_{22} \\
\frac{A_{21}-B_{2}\left(C_{f} B_{2}\right)^{-1} C_{f} A_{21}}{\varepsilon} & \frac{A_{22}-B_{2}\left(C_{f} B_{2}\right)^{-1} C_{f} A_{22}}{\varepsilon}
\end{array}\right]\left[\begin{array}{l}
x \\
z
\end{array}\right] .
$$

Equation (4.24) represents the sliding dynamics. The reduced order eigenvalues must be in the left half plane and satisfy the desired dynamics for the full order system equation.

\subsection{Illustrative Example}

Consider a magnetic tape control system [6] given by

$$
\begin{aligned}
& {\left[\begin{array}{l}
\dot{x}_{1} \\
\dot{x}_{2} \\
\varepsilon \dot{z}_{1} \\
\varepsilon \dot{z}_{2}
\end{array}\right]=\left[\begin{array}{cccc}
0 & 0.4 & 0 & 0 \\
0 & 0 & 0.345 & 0 \\
0 & -0.524 & -0.465 & 0.262 \\
0 & 0 & 0 & -1
\end{array}\right]\left[\begin{array}{l}
x_{1} \\
x_{2} \\
z_{1} \\
z_{2}
\end{array}\right]+\left[\begin{array}{l}
0 \\
0 \\
0 \\
1
\end{array}\right] u} \\
& y=\left[\begin{array}{llll}
1 & 0 & 0 & 0 \\
0 & 0 & 1 & 0
\end{array}\right]\left[\begin{array}{l}
x_{1} \\
x_{2} \\
z_{1} \\
z_{2}
\end{array}\right] .
\end{aligned}
$$

This can be put in standard singularly perturbed form as,

$$
\begin{aligned}
& \dot{x}=A_{11} x+A_{12} z+B_{1} u, \\
& \varepsilon \dot{z}=A_{12} x+A_{22} z+B_{2} u,
\end{aligned}
$$

where $\quad A_{I 1}=\left[\begin{array}{cc}0 & 0.4 \\ 0 & 0\end{array}\right], \quad A_{I 2}=\left[\begin{array}{cc}0 & 0 \\ 0.345 & 0\end{array}\right], \quad A_{I 2}=\left[\begin{array}{cc}0 & -0.524 \\ 0 & 0\end{array}\right]$,

$$
\begin{aligned}
& A_{22}=\left[\begin{array}{cc}
-0.465 & 0.262 \\
0 & -1
\end{array}\right] \\
& B_{1}=\left[\begin{array}{l}
0 \\
0
\end{array}\right], B_{2}=\left[\begin{array}{l}
0 \\
1
\end{array}\right] \text { and } \varepsilon=0.1 .
\end{aligned}
$$


The fast subsystem is given by equation (4.6). For our example it takes the form

$$
\varepsilon \dot{\eta}=A_{22} \boldsymbol{\eta}+B_{2} u_{f}
$$

The control law can be obtained for the slow and fast subsystems using the method of equivalent control described in the preceding section. The control law for the slow system is given by

$$
u_{s}=-\left(C_{s} B_{s}\right)^{-1} C_{s} A_{s} x_{s}-\left(C_{s} B_{s}\right)^{-1} \mu_{I} \operatorname{sgn}\left(C_{s} x_{s}\right)
$$

The second order system is reduced to first order in the sliding mode. By using equation (4.10) and the symbolic toolbox in Matlab and by defining the slow surface parameters as $C_{s}=\left[\begin{array}{ll}C_{1} & C_{2}\end{array}\right]$ we use the "syms" command to obtain $A_{e q}$ as,

$$
A_{e q}=\left[\begin{array}{cc}
0 & \frac{2}{5} \\
0 & -\frac{2}{5} \frac{C_{1}}{C_{2}}
\end{array}\right]
$$

The eigenvalues of the above equation are $\lambda_{1}=0$ and $\lambda_{2}=-\frac{2 C_{1}}{5 C_{2}}$. Selecting the time constant for the system in sliding mode to be $T_{s}=2.5$ therefore we need $\lambda_{2}=-0.4$, we choose the surface parameters to be $C_{s}=\left[\begin{array}{ll}1 & 1\end{array}\right]$. The control law for the slow subsystem will take the form,

$$
u_{s}=-\left[\begin{array}{ll}
0 & 0.0577
\end{array}\right]\left[\begin{array}{l}
x_{1} \\
x_{2}
\end{array}\right]-5.144 \times 1 \times \operatorname{sgn}\left(C_{s} x\right)
$$

The fast subsystem surface parameters are chosen based on the same analysis as for the slow subsystem. For the fast subsystem the control law has the form,

$$
u_{f}=-\left(C_{f} B_{2}\right)^{-1} C_{f} A_{22} \eta-\left(C_{f} B_{2}\right)^{-1} \mu_{2} \operatorname{sgn}\left(C_{f} \eta\right)
$$


equation (4.10) then takes the form,

$\dot{\eta}_{e q}=\left[I-B_{2}\left(C_{f} B_{2}\right)^{-I} C_{f}\right] A_{22} \eta_{e q}=A_{e q} \eta_{e q}$

where $\eta_{e q}$ is the fast state in the sliding mode. Using the symbolic toolbox and command "syms" then equation (4.32) can be solved as,

$$
A_{e q}=\left[\begin{array}{cc}
\frac{-93}{200} & \frac{-131}{500} \\
\frac{93 C_{1}}{200 C_{2}} & \frac{-131 C_{1}}{500 C_{2}}
\end{array}\right]
$$

The eigenvalues for the fast equivalent system given by equation (4.33) are,

$$
\lambda_{1}=0 \text { and } \lambda_{2}=\frac{-\left(465 C_{2}+262 C_{1}\right)}{1000 C_{2}}
$$

Based on the above analysis we could choose $C_{f}=\left[\begin{array}{ll}3 & 1\end{array}\right]$ and the corresponding control law will have the form,

$$
u_{f}=\left[\begin{array}{ll}
-1.395 & -0.214
\end{array}\right] \eta-2 \operatorname{sgn}\left(S_{f}\right)
$$

Figure 4.1 illustrates the closed loop time response of the full order system. We notice from Figure 4.1 that the chattering in the slow subsystem has a great effect on the fast subsystem. The performance is suffering from oscillations. To decrease the amount of chattering, a saturation function is used to replace the signum function in the discontinuous control law. This means that we apply a boundary layer around the sliding surface as explained by the preceding section (2.3). We choose the thickness of the boundary layer to be $\phi=0.15$. We iteratively change the thickness of the boundary layer until reaching the best performance in the slow state with smallest thickness. The output performance of the full order system is greatly improved when chattering is decreased. This can be seen in Figure 4.2. This example illustrates the use of two sliding mode 
controllers to control the full order singularly perturbed model. It also demonstrates clearly the chattering effect due to the high frequency switching on the response of the fast and slow state.

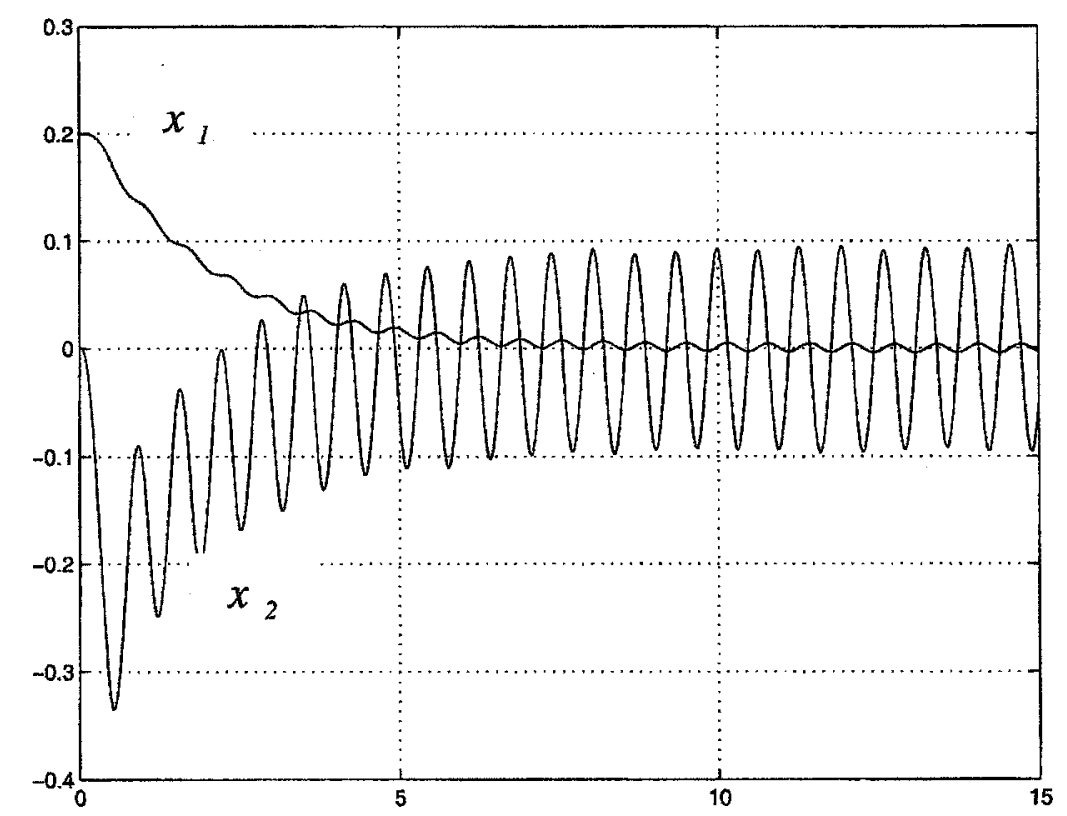

Figure 4.1.a: Full order closed loop time response (slow subsystem) 


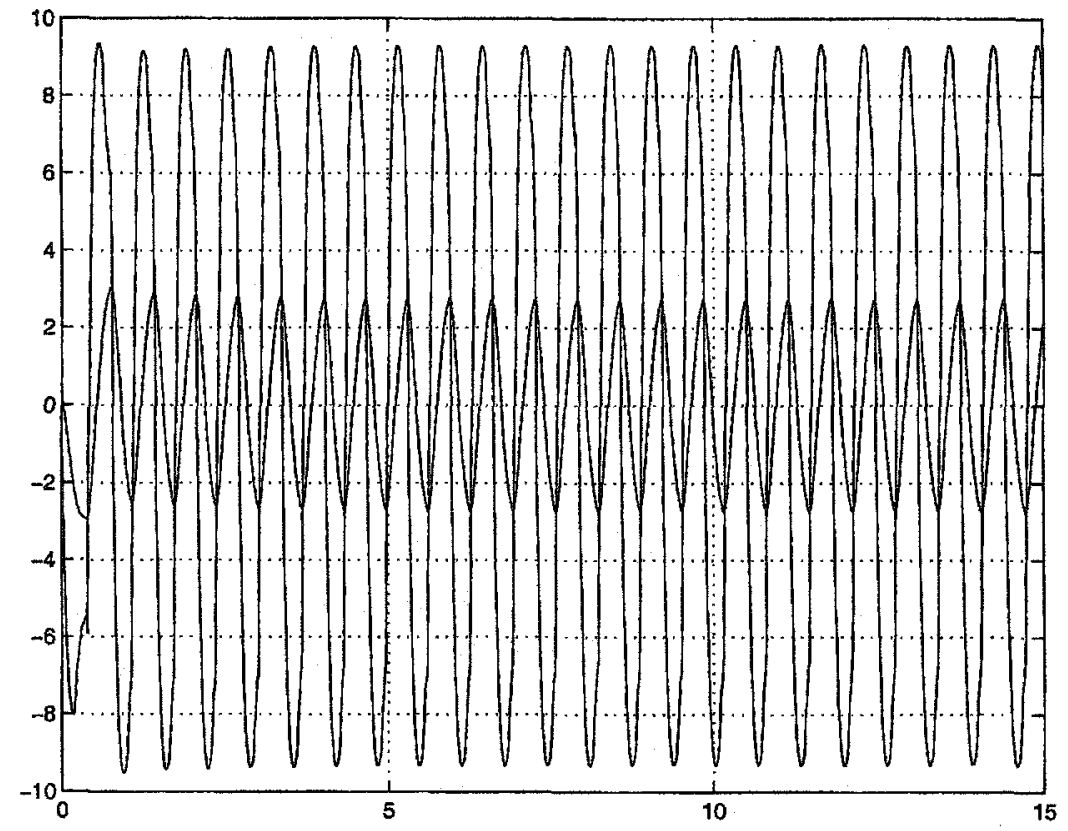

Figure 4.1.b: Full order closed loop time response (fast subsystem)

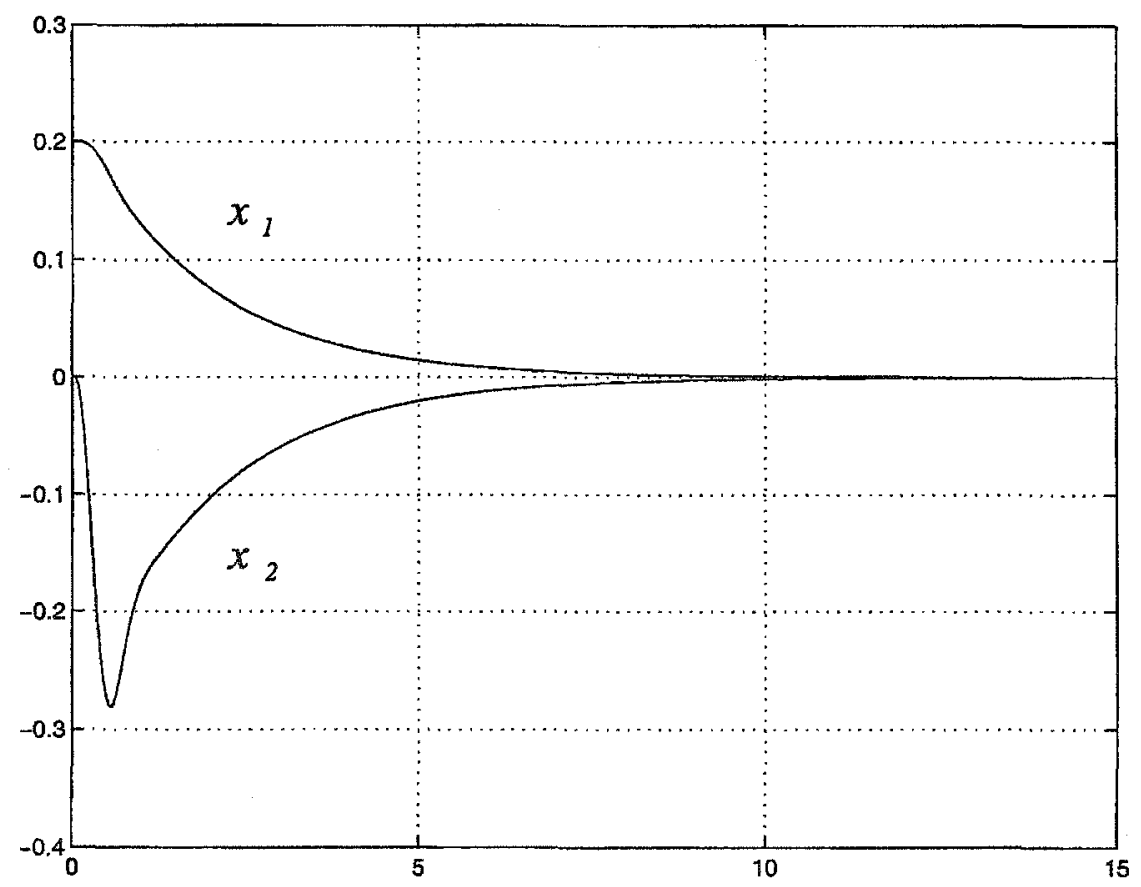

Figure 4.2.a: Full order closed loop time response with chattering reduction in the slow subsystem 


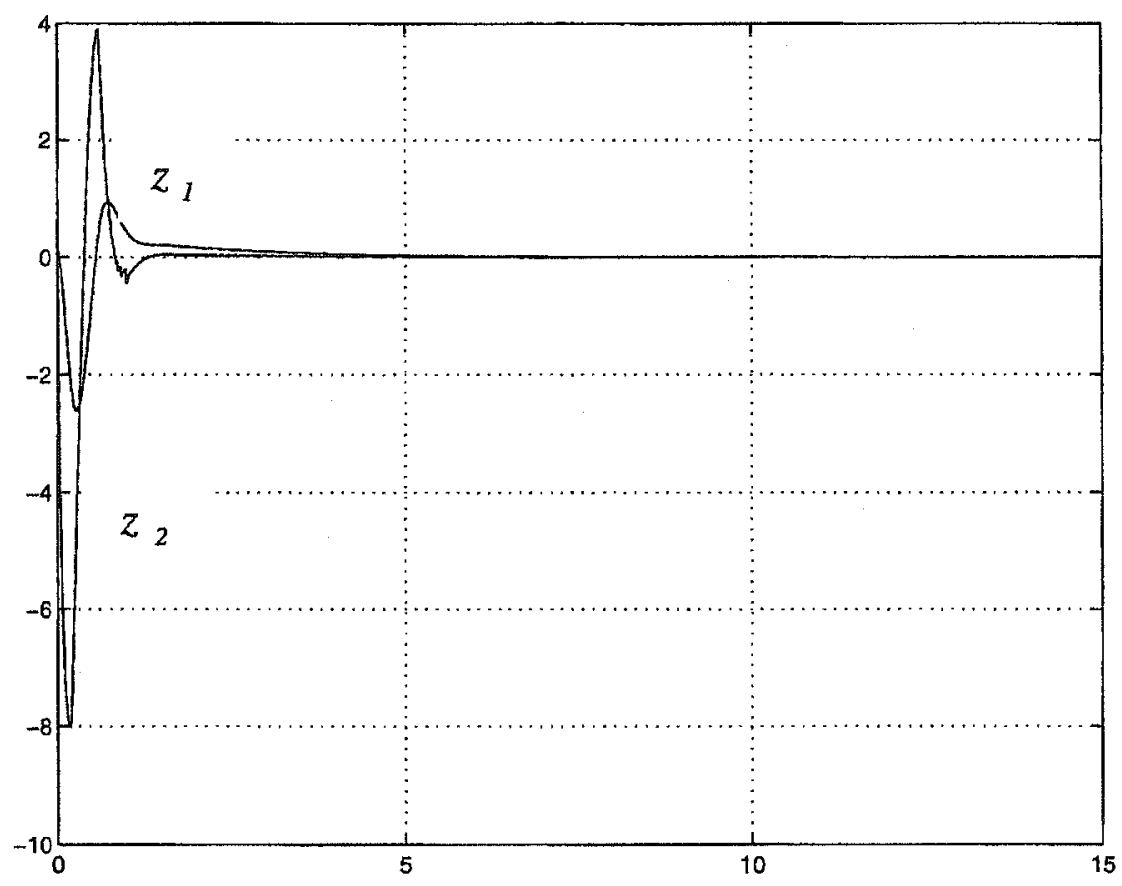

Figure 4.2.b: Full order closed loop time response with chattering reduction in the slow subsystem. 


\section{Chapter 5 New Sliding Mode Controllers for}

\section{Singularly Perturbed Systems}

\subsection{Introduction}

A basic issue in the control of linear time invariant systems is the investigation of the effect of modeling errors on the performance of the control system. Generally speaking there are two models, the actual model and the design model. The actual model has the closest reliability to the actual physical system. The design model is used to derive a control strategy and represents a simplified version of the actual model where some of the modes may be neglected or some of the parameters are fixed at nominal values.

Singular perturbation analysis offers a good method to study the effect of discrepancy between the two models on the performance of the control system. In fact a singularly perturbed system often occurs naturally because of the presence of small parasitic parameters multiplying the time derivatives of some of the system states. The main idea, as described before in chapter 3 , is to obtain two reduced order subsystems using appropriate decoupling techniques from the full order singularly perturbed model. These two reduced subsystems are usually called the slow and the fast subsystems.

In this chapter, we will the use the singular perturbation model formulation to improve the design process of the sliding mode controller. This can be done by studying the effect of the fast subsystem on the sliding mode controller implemented for the slow subsystem. The result from this investigation will help us to choose the appropriate 
parameters for the sliding mode controller. The proper selection of these parameters will avoid the excitation of high frequency dynamics, which may cause instability as shown in Figure 5.2, and Figure 5.5. Two main areas have been investigated.

First, we require that the controller based on the slow subsystem can stabilize the system for a sufficiently small value of $\varepsilon$. There is a strong relationship between the surface parameter in the sliding mode control and the value of the perturbation parameter $\varepsilon$. It is essential to study the stability in this case to determine the maximum value of $\varepsilon$ (i.e. $\varepsilon^{*}$ ) that will maintain the stability of the full order system controlled by a single sliding mode controller.

Second, sliding mode controllers have two phases in the controller design: one is the reaching phase where the state moves from arbitrary initial conditions towards the sliding surface, and the other is the sliding phase where the state follows the sliding surface. Consequently, choosing sliding surfaces plays an important role in the response of sliding mode control systems. It is desired that the surface parameters satisfy two conflicting objectives, one is a fast closed loop response and the second is a stable closed loop system which is robust against unmodeled high frequency dynamics. This problem is discussed in detail in chapter 6.

In this chapter, a single sliding mode controller for the full order singularly perturbed system, the stability bounds for the closed loop singularly perturbed system and the approximation error obtained from the decoupling method are investigated. This chapter is organized as follows, in section 5.2 a diagonalization method is used to design a double sliding mode controller and single sliding mode controlier for the full order system. It is shown that this method overcomes the approximation error that quasi steady state causes. 
Section 5.3 shows the simulation results for an illustrative example using the two decoupling methods and a comparison between them. Section 5.4 discusses the stability of a single sliding mode controller and obtains the maximum value of perturbation parameter $\varepsilon^{*}$ such that stability will be preserved. Section 5.5 describes an illustrative example where we obtain the maximum value of perturbed parameter $\varepsilon^{*}$.

\subsection{Accurate reduced order singularly perturbed model for}

\section{sliding mode control}

Consider a system in the following form,

$\dot{x}=A_{11} x+A_{12} z+B_{1} u$,

$\varepsilon \dot{z}=A_{21} x+A_{22} z+B_{2} u$

where $x \in R^{n}, z \in R^{m}, u \in R^{r}$ and $\varepsilon$ is a small positive parameter. This is a form for standard linear time invariant singularly perturbed systems. The slow and fast subsystem can be obtained using the diagonalization approach as discussed in chapter 3 . The system has the required block diagonal or decoupled form,

$\left[\begin{array}{l}\dot{x_{s}} \\ \dot{x_{f}}\end{array}\right]=\left[\begin{array}{cc}A_{s} & 0 \\ 0 & A_{f}\end{array}\right]\left[\begin{array}{l}x_{s} \\ x_{f}\end{array}\right]+\left[\begin{array}{l}B_{s} \\ B_{f}\end{array}\right] u$

where the slow and the fast variables $x_{s}$ and $x_{f}$ can be solved independently of each other. The eigenvalues of $A_{s}$ are the same as the slow poles of the original system [4] [30]. Similarly the eigenvalues of $A_{f}$ are the same as the fast poles of the full order model. The matrix $L$ is dependent on the perturbation parameter $\varepsilon$ where $L$ is one of the matrices needed for diagonalization as discussed in chapter 3. Letting $\varepsilon \rightarrow 0$ gives us 
$L=L_{0}=A_{22}^{-1} A_{21}$ which transforms the diagonal model into the same as the quasi steady state model described in chapter $3\left(A_{s}=A_{0}\right)$. Changing the value of the perturbation parameter $\varepsilon$ to a small non-zero value and checking the difference between the eigenvalues of $A_{s}$ and $A_{0}$ gives us an indication if the quasi steady state is a valid approximation or not. One may expect that if $A_{s}$ is very sensitive to the value of $\varepsilon$ then using $A_{0}$ instead of $A_{s}$ may cause large errors in the approximation of the slow poles of the original system. Similarly, if $A_{21}$ and $A_{22}$ are dependent on $\varepsilon$, the approximation of the fast system where we set $A_{f}=A_{22}$ may lead to an unstable system or increase the steady state error of the closed loop system [30].

Based on previous analysis, the diagonalization method is preferred instead of quasi steady state to decouple the full order system. This is advantageous especially when the value of $\varepsilon$ is not small and the quasi steady state model fails to describe the properties of the full order model correctly.

\subsubsection{Sliding mode control design using diagonalization method}

In this section, two sliding mode controllers are designed to control the full order singularly perturbed system. This is what is proposed in the literature [22][50], but in our case we will use the diagonalization method instead of quasi steady state. The reason for doing this is to compare between the quasi steady state and diagonalization method in this case. Then, we use the diagonalization method to design a single sliding mode controller for the full order singularly perturbed system, which has never been done before.

The design of the sliding mode controller for the full order system is done in two stages. First, a slow controller is designed based on the slow subsystem. Second, the fast 
controller is designed based on the fast subsystem. The composite of these controllers is used to control the full order model. This is similar to what was done before and discussed in the last chapter. However, in the following sections we will use the diagonalization method instead of the quasi steady state to obtain the decoupled system.

\subsubsection{Slow sliding mode control design}

The slow sliding mode controller is designed using the slow subsystem obtained from the diagonalized full order singularly perturbed system given by equation (5.3). The equivalent control method [10], described in chapter 2 section 2.2 , is used to design such a controller. We define the linear switching surface as $S_{s}=C_{s} x_{s}$. The equivalent control law ensures that the system remains on the sliding surface and has the form,

$u_{e q}=-\left[\left(C_{s} B_{s}\right)^{-1} C_{s} A_{s}\right] x_{s}$.

Using this control law in the slow subsystem obtained from the block diagonalized reduced system (5.3) we then obtain,

$\dot{x}_{e q s}=\left[I-B_{s}\left(C_{s} B_{s}\right)^{-1} C_{s}\right] A_{s} x_{e q s}$.

To guarantee local stability for this controller we must ensure that the $n-r$ eigenvalues of the equivalent system given by equation (5.5) have negative real parts. A Lyapunov function is used to determine the discontinuous control law $\left(u_{N}\right)$ that will satisfy the reaching condition. This will ensure the global asymptotic stability of the closed loop system.

The control law, for the slow subsystem, has the form,

$$
u_{s}=u_{e q}+u_{N}=-\left[\left(C_{s} B_{s}\right)^{-1} C_{s}\right] A_{s} x_{s}-\left(C_{s} B_{s}\right)^{-1} \mu_{l} \operatorname{sgn}\left(S_{s}\right)
$$


where $u_{N}$ is a discontinuous control action that drives the state to the sliding surface and $\mu_{1}$ is an arbitrary positive constant.

\subsubsection{Fast sliding mode controller design}

The same argument used to design the slow sliding mode controller can be used to design the fast sliding mode controller. Define the linear fast switching surface $S_{f}=C_{f} x_{f}$. The equivalent control method is used to determine the control law for the fast subsystem which can be obtained from equation (5.3). The fast subsystem in the sliding mode is given by,

$\dot{x}_{e q f}=\left[I-B_{f}\left(C_{f} B_{f}\right)^{-I} C_{f}\right] A_{f} x_{e q f}$

The combined control law is given by,

$u_{f}=-\left[\left(C_{f} B_{f}\right)^{-1} C_{f}\right] A_{f} x_{f}-\left(C_{f} B_{f}\right)^{-1} \mu_{2} \operatorname{sgn}\left(S_{f}\right)$

which ensures global asymptotic stability for fast the subsystem. The control law for the full order model will be the composite of the slow and fast controllers,

$u=u_{s}\left(x_{s}\right)+u_{f}\left(x_{f}\right)$.

Satisfying the reaching condition for the reduced order model implies that the reaching condition for the full order model is also satisfied [22]. Since the eigenvalues of the reduced order subsystems are the same as the full order model this means that the linear transformation preserves the stability condition for the closed loop system. Taking the stability analysis proposed by Kokotovic [30] into account, we could say that the full order model is globally stable for $\varepsilon \in\left(0, \varepsilon^{*}\right)$. 


\subsubsection{Slow sliding mode controller for the full order model}

In this section, a single sliding mode controller is proposed to control the full order model. We will consider that the unmodeled high frequency dynamics are represented by the fast subsystem. The main assumption is that the matrix $A_{22}$ is stable while the full order model given by equation (5.1) and equation (5.2) may be unstable at some specific value of $\varepsilon$. The surface parameter for the single sliding mode controller is chosen based on Slotine's [48][49] recommendation where the natural frequency of the system in the sliding mode must be smaller than the lowest frequency of the fast subsystem. The system in sliding mode has the form,

$\dot{x}_{e q s}=\left[I-B_{s}\left(C_{s} B_{s}\right)^{-1} C_{s}\right] A_{s} x_{e q s}$

The control law is chosen based on an equivalent control method and has the following form,

$u_{s}=-\left[\left(C_{s} B_{s}\right)^{-1} C_{s}\right] A_{s} x_{s}-\left(C_{s} B_{s}\right)^{-1} \mu_{1} \operatorname{sgn}\left(S_{s}\right)$

\subsection{Illustrative examples of diagonalization method}

\section{Example (5.1)}

Consider a magnetic tape control system [6] given by

$$
\left[\begin{array}{c}
\dot{x}_{1} \\
\dot{x_{2}} \\
\varepsilon \dot{z}_{1} \\
\varepsilon \dot{z}_{2}
\end{array}\right]=\left[\begin{array}{cccc}
0 & 0.4 & 0 & 0 \\
0 & 0 & 0.345 & 0 \\
0 & -0.524 & -0.465 & 0.262 \\
0 & 0 & 0 & -1
\end{array}\right]\left[\begin{array}{l}
x_{1} \\
x_{2} \\
z_{1} \\
z_{2}
\end{array}\right]+\left[\begin{array}{l}
0 \\
0 \\
0 \\
1
\end{array}\right] u
$$


$y=\left[\begin{array}{llll}1 & 0 & 0 & 0 \\ 0 & 0 & 1 & 0\end{array}\right]\left[\begin{array}{l}x_{1} \\ x_{2} \\ z_{1} \\ z_{2}\end{array}\right]$

This can be put in standard singularly perturbed form as,

$\dot{x}=A_{11} x+A_{12} z+B_{1} u$

$\varepsilon \dot{z}=A_{12} x+A_{22} z+B_{2} u$

where $\quad A_{11}=\left[\begin{array}{cc}0 & 0.4 \\ 0 & 0\end{array}\right], \quad A_{12}=\left[\begin{array}{cc}0 & 0 \\ 0.345 & 0\end{array}\right], \quad A_{12}=\left[\begin{array}{cc}0 & -0.524 \\ 0 & 0\end{array}\right]$,

$A_{22}=\left[\begin{array}{cc}-0.465 & 0.262 \\ 0 & -1\end{array}\right]$

$B_{1}=\left[\begin{array}{l}0 \\ 0\end{array}\right], B_{2}=\left[\begin{array}{l}0 \\ 1\end{array}\right]$ and $\varepsilon=0.1$

The first $2 \times 2$ transformation matrix $L$ has to satisfy,

$R(L)=A_{21}-A_{22} L+\varepsilon L A_{11}-\varepsilon L A_{12} L=0$.

This gives us the value of $L$ as,

$L=\left[\begin{array}{cc}0 & 1.2412 \\ 0 & 0\end{array}\right]$

The second $2 \times 2$ transformation matrix $M$ has to satisfy

$S(M)=\varepsilon\left(A_{11}-A_{12} L\right) M-M\left(A_{22}+\varepsilon L A_{12}\right)+A_{12}=0$.

Similarly this gives us the value of $M$ as

$M=\left[\begin{array}{cc}0.0862 & 0.0325 \\ -0.9094 & -0.2489\end{array}\right]$

The slow subsystem is given by 
$\dot{x_{s}}=A_{s} x_{s}+B_{s} u_{s}$

where $A_{s}=A_{11}-A_{12} L=\left[\begin{array}{cc}0 & 0.4 \\ 0 & -0.4282\end{array}\right]$ and $B_{s}=\left[\begin{array}{c}-0.0325 \\ 0.2489\end{array}\right]$.

The fast subsystem can be described by

$\varepsilon \dot{x}_{f}=A_{f} x_{f}+B_{f} u_{f}$

where $A_{f}=\left[\begin{array}{cc}-0.4222 & 0.262 \\ 0 & -1\end{array}\right]$ and $B_{f}=\left[\begin{array}{l}0 \\ 1\end{array}\right]$.

We notice that the eigenvalues of the reduced systems are the same as the eigenvalues of the full order model which are $\lambda_{1}=0, \lambda_{2}=-0.4282, \lambda_{3}=-4.22$ and $\lambda_{4}=-10$. We design two sliding mode controllers based on the slow and fast subsystems. We design the controllers based on the equivalent control method using the same design steps as shown in chapter 4 section 4.5 . The slow system in sliding mode is represented by equation(5.5), which has eigenvalues equal to,

$$
\lambda_{1}=0 \text { and } \lambda_{2}=\frac{851.6 C_{1}}{325 C_{1}-2489 C_{2}}
$$

Choosing the time constant for the slow subsystem in the sliding mode to be $T_{s}=2$, the corresponding sliding surface parameters for the slow subsystem are $C_{s}=\left[\begin{array}{ll}1.3 & 1\end{array}\right]$. Thus substituting into equation (5.6) the control law based on the diagonalized system will be,

$u_{s}=\left[\begin{array}{ll}0 & 0.4442\end{array}\right] x_{s}-4.839 \operatorname{sgn}\left(S_{s}\right)$

Recall from chapter 4 , equation (4.13) the control law based on the quasi steady state method will be,

$$
u_{s}=\left[\begin{array}{ll}
0 & 0.6751
\end{array}\right] x_{s}-5.144 \operatorname{sgn}\left(S_{s}\right)
$$


Similarly the fast subsystem in sliding mode is represented by equation (5.7). Choosing the time constant for the fast subsystem in the sliding mode to be $T_{f}=12.5$. The corresponding sliding surface for the fast subsystem is $C_{f}=\left[\begin{array}{ll}3 & 1\end{array}\right]$ thus substituting into equation (5.8) the control law based on the diagonalized system is,

$u_{f}=\left[\begin{array}{ll}-1.2665 & -0.214\end{array}\right] x_{f}-2 \operatorname{sgn}\left(S_{f}\right)$

Recall from chapter 4, equation (4.19) the control law based on the quasi steady state method will be,

$u_{f}=\left[\begin{array}{ll}-1.395 & -0.214\end{array}\right] x_{f}-2 \operatorname{sgn}\left(S_{f}\right)$

The control law for the full order model is the composite of the slow and fast controls,

$$
u=u_{s}\left(x_{s}\right)+u_{f}\left(x_{f}\right)
$$

A boundary layer around the sliding surfaces is introduced to decrease the chattering effect. Similar to what was done in chapter 4 an iteration procedure is used to determine the best selection of boundary layer thickness. The thickness of the boundary layer is chosen to be $\phi=0.1$. Figure 5.1 illustrates the closed loop time response of the full order system. It is observed that the absolute value of the error between the desired response and zeroth model response is larger than the absolute error between the desired response and the proposed sliding mode controller response based on diagonalization. The desired response is defined as the initial condition response of the reference model system for the slow subsystem and is given by,

$$
\frac{Y(s)}{x(s)}=\frac{0.0778}{s^{2}+0.8 s+0.16}
$$


We chose the sliding surface parameters based on the time response of this model for both the zeroth order model and the diagonalization model. Similarly we choose the fast subsystem reference model as,

$\frac{Y(s)}{x(s)}=\frac{26.2}{s^{2}+10 s+25}$

The responses in these figures are initial condition responses. This response is used in all the examples in this chapter and in the next two chapters.

It is a fact that the first requirement of any control strategy is to stabilize the closed loop system. In control theory it is well known that we can design a controller based on the reduced order system given by equation (5.5) if we neglect the fast modes. Neglecting fast modes can only be done if $\varepsilon$ is sufficiently small and the fast eigenvalues are stable.

We will design a single sliding mode controller for the slow subsystem given that the fast subsystem in our model is stable. In this controller we want to obtain a relatively fast response without exciting the high frequency dynamics that may lead to an unstable closed loop system. The approach is to let the natural frequency of the system in the sliding mode be smaller than the lowest frequency of the fast modes (high frequency modes). The eigenvalues for the fast subsystem are, $\lambda_{f 1}=-4.22$ and $\lambda_{f 2}=-10$. Therefore, we will choose desired eigenvalue as $\lambda_{d}=-4$. The system in sliding mode is represented by equation (5.10). The eigenvalues for the system represented by equation (5.10) can be calculated using the "syms" command in Matlab. The eigenvalues are,

$$
\lambda_{1}=0 \text { and } \lambda_{2}=\frac{851.6 C_{1}}{325 C_{1}-2489 C_{2}}
$$

As a result, the surface parameter is chosen to be 
$C=\left[\begin{array}{ll}4 & 1\end{array}\right]$

This selection of surface parameters results in an unstable system as shown in Figure 5.2. A smaller absolute value of $\lambda_{d}$ will enable us to get a stable response. We choose the surface parameter to be,

$C=\left[\begin{array}{ll}3 & 1\end{array}\right]$

which gives us the response shown in Figure 5.3. The motion along the sliding surface will result in a closed loop controller with a lower bandwidth and longer time response. The significance of this result is that the slope of the sliding surface has an effect on the stability of the full order system. Figure 5.4 shows the phase plane plot of the slow states, which illustrate the sliding surface and the boundary layer around it. The control law has the form,

$$
u_{s}=\left[\begin{array}{ll}
0 & 5.0977
\end{array}\right] x-6.065 \mathrm{sat}\left(\frac{S_{s}}{0.1}\right)
$$

Figure 5.3 illustrates the closed loop time response of the full order system using a single sliding mode controller. It is shown that it is possible to use one sliding mode controller to control the full order model for a singularly perturbed system given that the fast subsystem is stable and we choose the right surface parameters that do not cause excitation of the fast subsystem. The outputs of the controllers are shown in Figure 5.5. In this figure both composite controllers and single controller outputs are illustrated. Figure 5.5.b is focused on the first half second of Figure 5.5.a. In this simulation, the control effort required during initial transients to achieve the desired performance is lower in the case of using a single sliding mode controller. Lower control effort achieving the desired performance is an advantage since it avoids actuator saturation. 


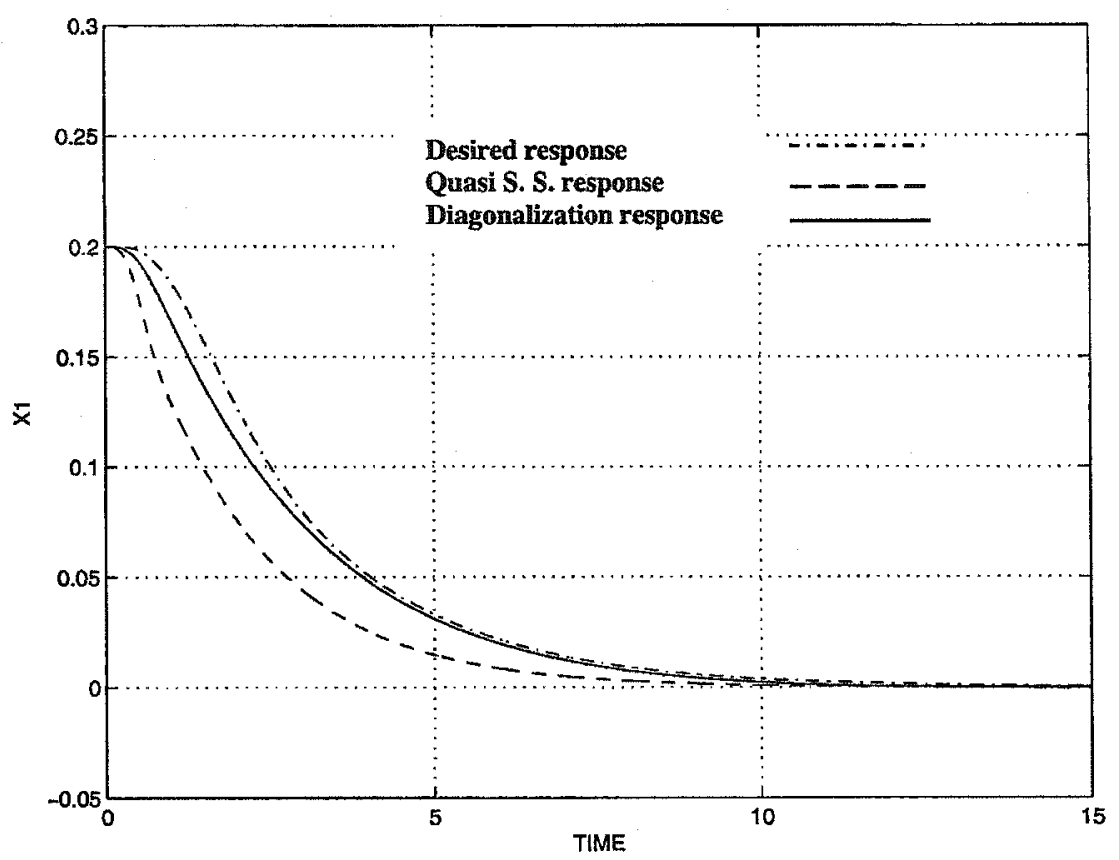

Figure 5.1.a: Full order closed loop time response for two sliding mode controllers (Slow state)

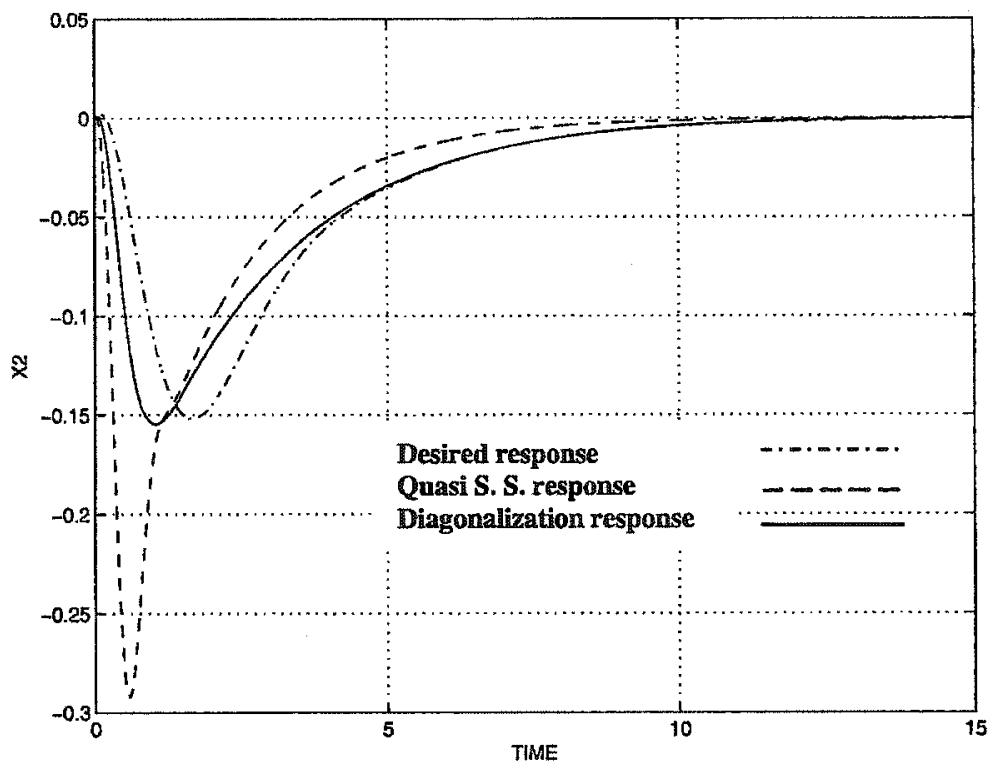

Figure 5.1.b: Full order closed loop time response for two sliding mode controllers (Slow state) 


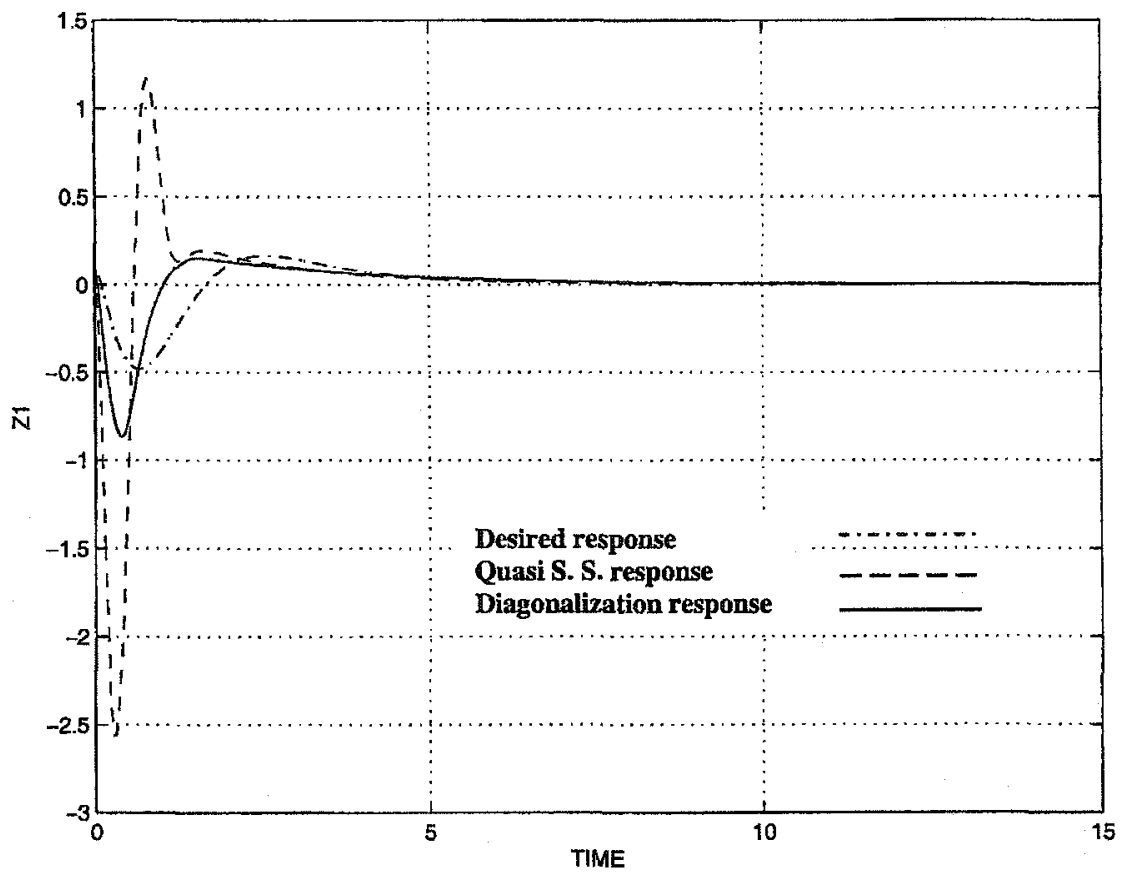

Figure 5.1.c: Full order closed loop time response for two sliding mode controllers (Fast state)

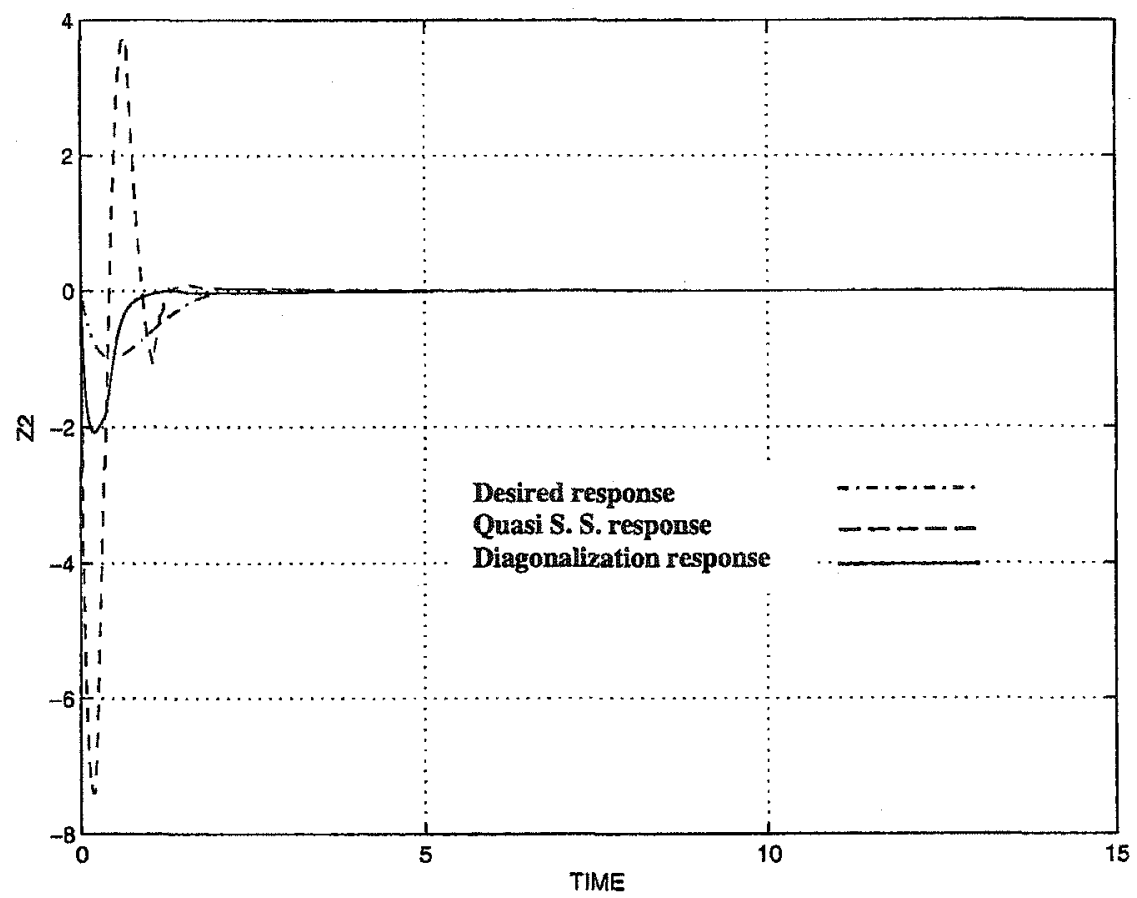

Figure 5.1.d: Full order closed loop time response for two sliding mode controllers (Fast state) 


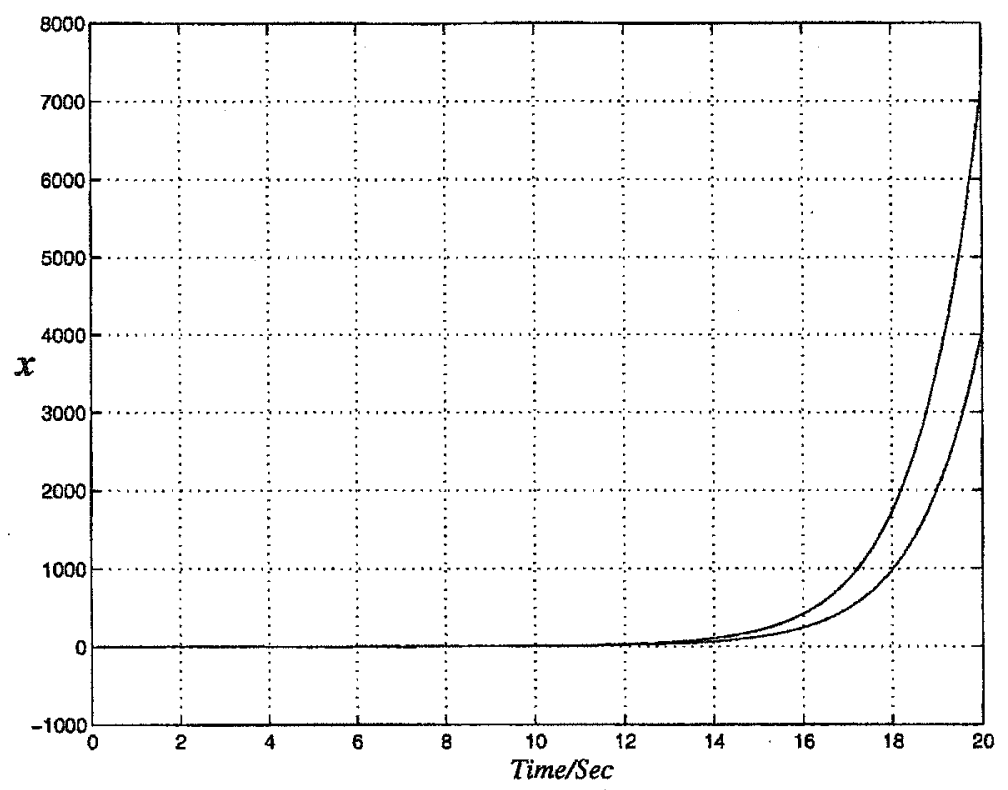

Figure 5.2.a: Full order closed loop time response for single sliding mode controller (Slow state) using diagonalization method

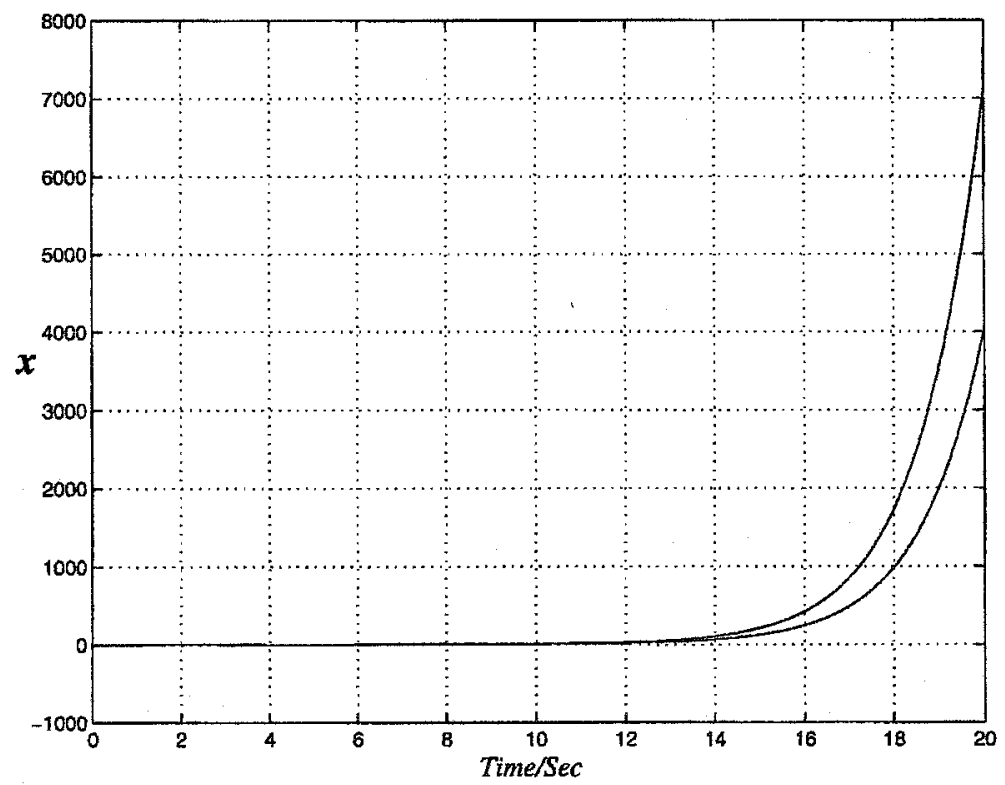

Figure 5.2.b: Full order closed loop time response for single sliding mode controller (Slow state) using quasi steady state method 


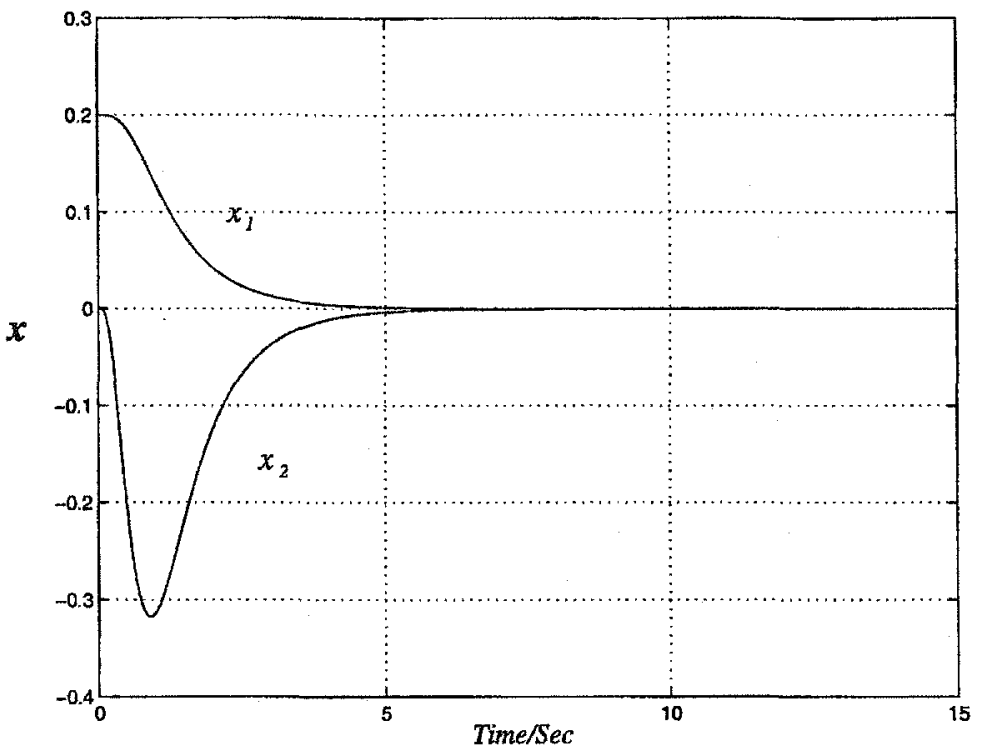

Figure 5.3.a: Full order closed loop time response for a single sliding mode controller (Slow state)

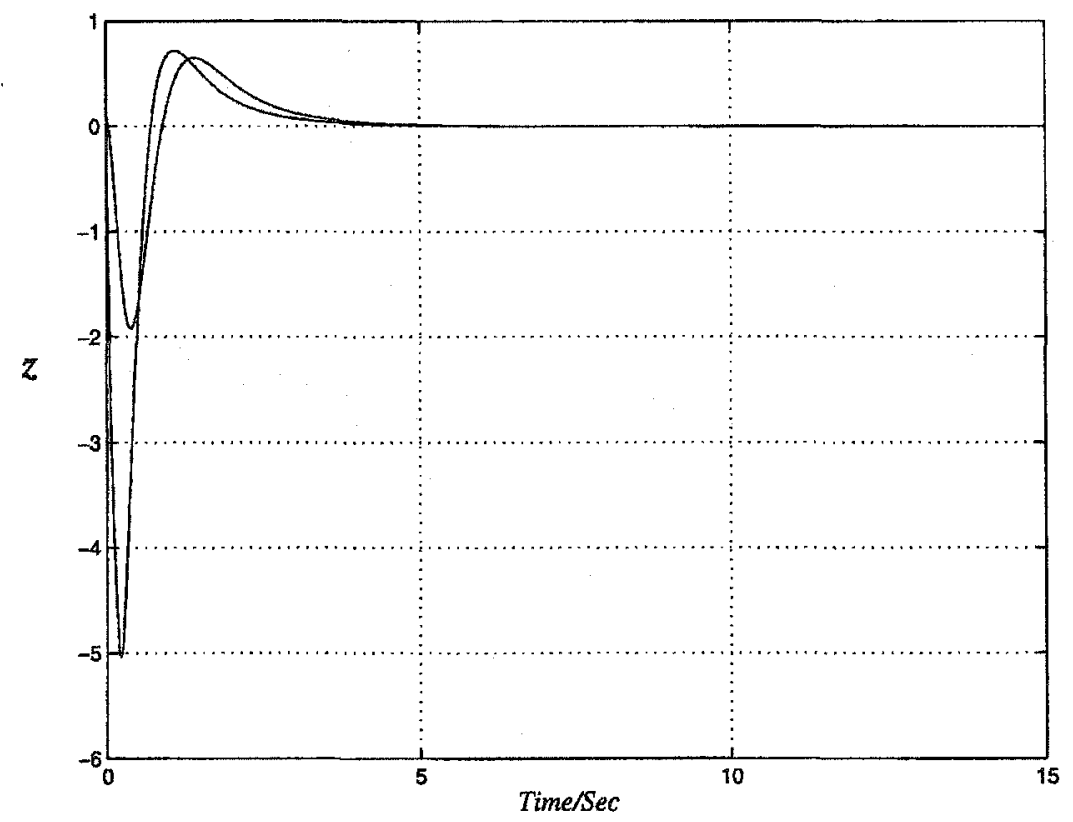

Figure 5.3.b: Full order closed loop time response for a single sliding mode controller (Fast state) 


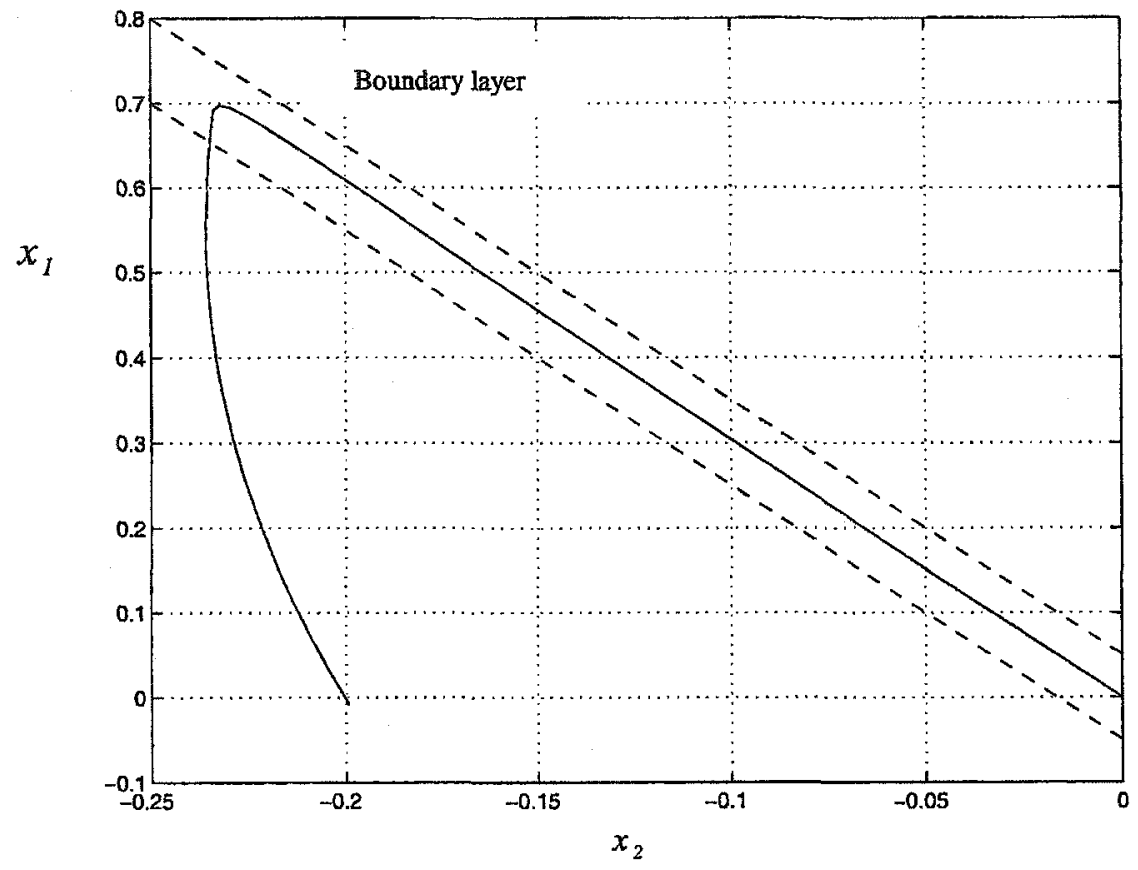

Figure 5.4: Phase plane for the single sliding mode controller with boundary layer

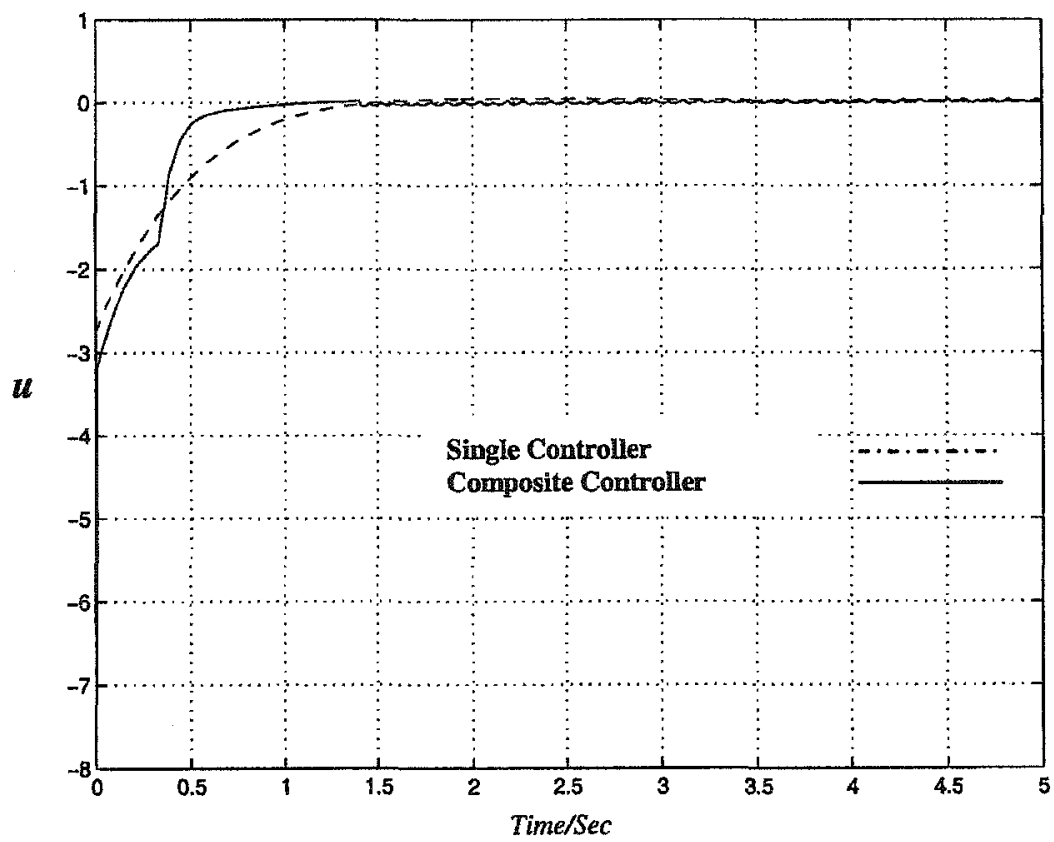

Figure 5.5.a: Closed loop time response for the control output (Composite and single controllers) 


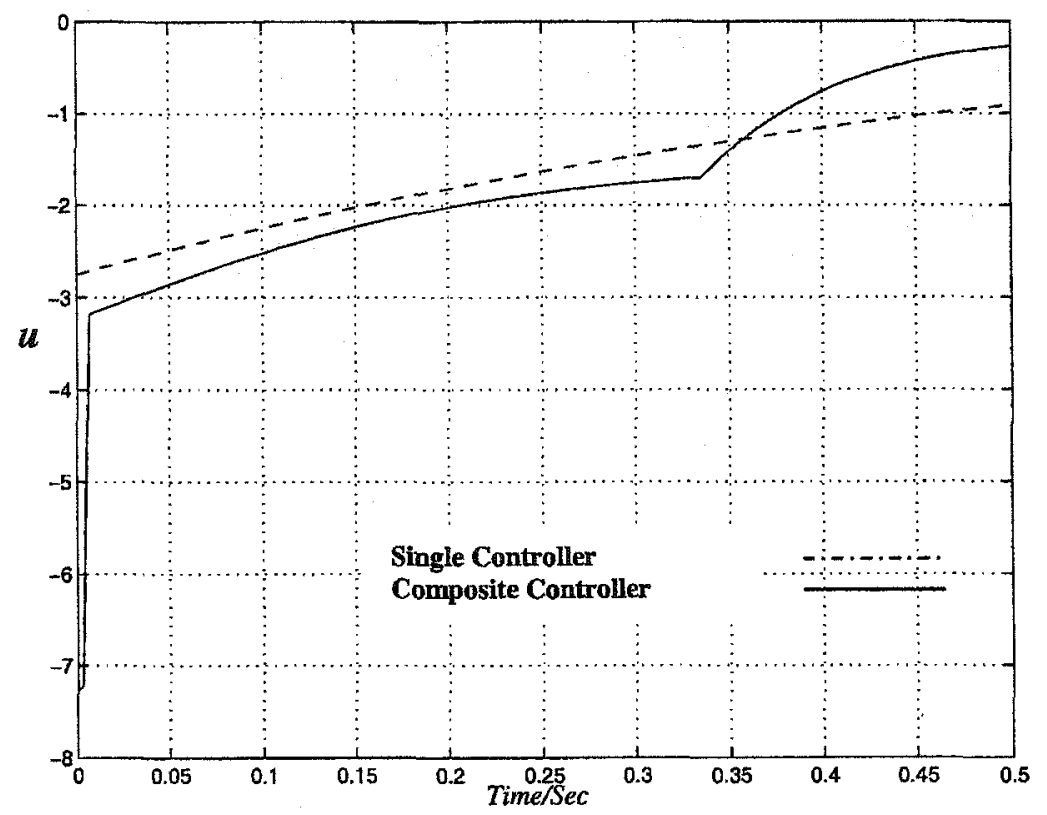

Figure 5.5.b: Closed loop time response for the control output focused on the first half second (Composite and single controllers)

\section{Example (5.2)}

This is the same example as explained in chapter 3 for a flexible joint manipulator. The system equations have the form,

$$
\left[\begin{array}{c}
\varepsilon \dot{\omega}_{1} \\
\dot{\omega}_{2} \\
\dot{\bar{\theta}}
\end{array}\right]=\left[\begin{array}{ccc}
-\varsigma_{1} & 0 & -K \\
0 & -\frac{S_{2}}{J_{m}} & \frac{K}{J_{m}} \\
1 & -1 & 0
\end{array}\right]\left[\begin{array}{c}
\omega_{1} \\
\omega_{2} \\
\bar{\theta}
\end{array}\right]+\left[\begin{array}{l}
1 \\
0 \\
0
\end{array}\right] u
$$

The above state equation can be put in state space form as:

$$
\dot{x}=\left[\begin{array}{c}
\dot{\omega}_{2} \\
\dot{\bar{\theta}}
\end{array}\right]=\left[\begin{array}{cc}
-\frac{S_{2}}{J_{m}} & \frac{K}{J_{m}} \\
-1 & 0
\end{array}\right]\left[\begin{array}{c}
\omega_{2} \\
\bar{\theta}
\end{array}\right]+\left[\begin{array}{l}
0 \\
1
\end{array}\right] \omega_{1}+\left[\begin{array}{l}
0 \\
0
\end{array}\right] u
$$




$$
\varepsilon \dot{z}=\varepsilon \dot{\omega}_{1}=\left[\begin{array}{ll}
0 & -K
\end{array}\right]\left[\begin{array}{c}
\omega_{2} \\
\bar{\theta}
\end{array}\right]+\left[-\varsigma_{1}\right] \omega_{1}+[1] u
$$

We design a sliding mode control law based on the slow subsystem taking into account the fast subsystem as unmodeled high frequency dynamics. The system in sliding mode is represented by equation (5.10). Similar to the previous procedure we chose the sliding surface as $C_{s}=\left[\begin{array}{ll}3.5 & 1\end{array}\right]$. Substituting into equation (5.11) the control law based on the quasi steady state method will be,

$$
u_{s}=\left[\begin{array}{ll}
-0.4125 & -11.8749
\end{array}\right] x_{s}-7.3992 \mathrm{sat}\left(\frac{S_{s}}{0.1}\right)
$$

The results from this approach are completely undesirable. The unmodeled high frequency dynamics are excited by the switching control law and result in chattering. Since there is no controller designed for the fast subsystem, the overall system diverges. This can be seen in Figure 5.6.

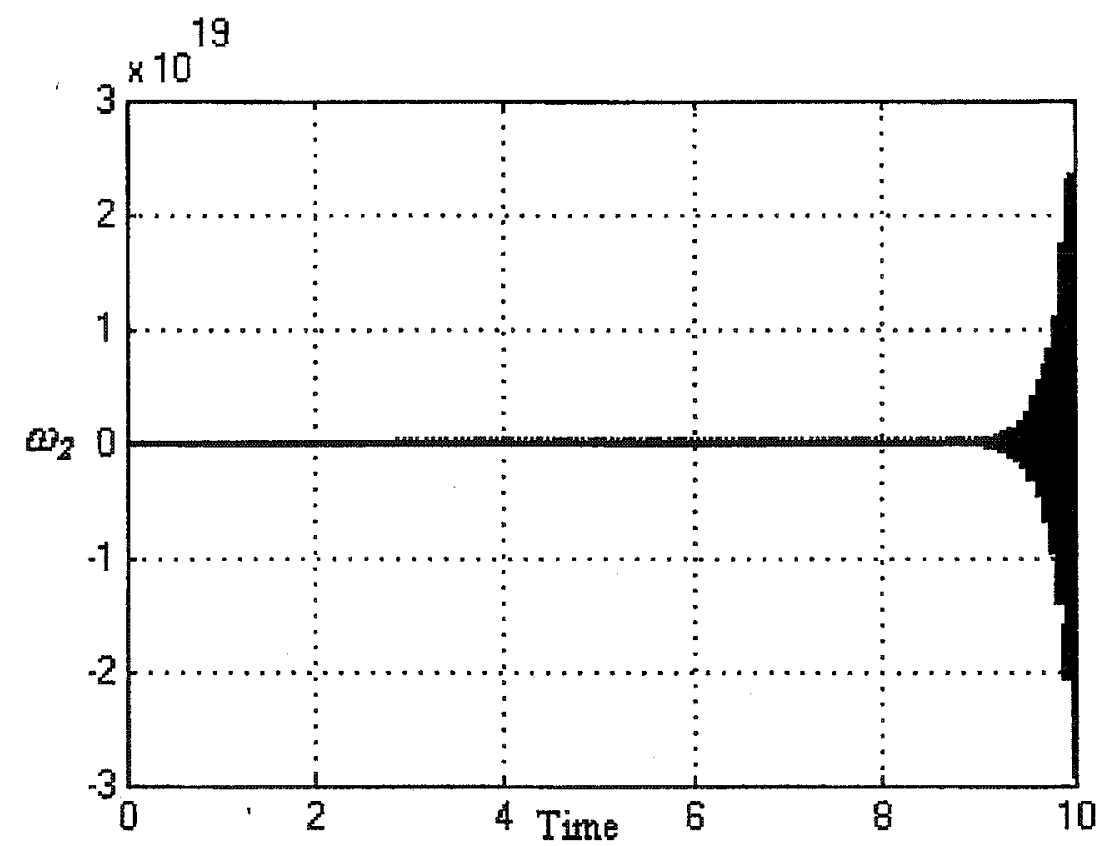

Figure 5.6.a: Full order closed loop time response for a single sliding mode controller (Slow State) 


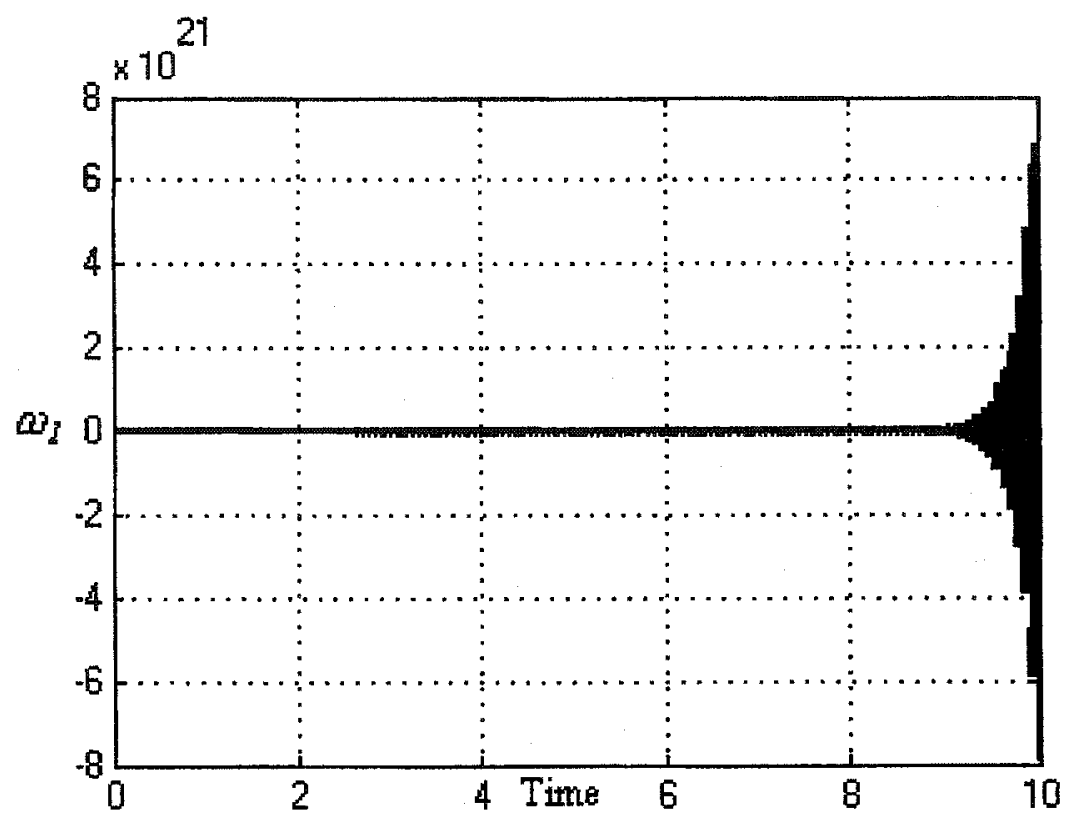

Figure 5.6.b: Full order closed loop time response for a single sliding mode controller (Fast state)

To overcome this problem we choose a smaller value for the surface parameters (i.e. decreasing the slope). This allows the single sliding mode controller to obtain a stable result for the full order singularly perturbed model. The high frequency dynamics are not being excited and the chattering effect is greatly reduced. Figure 5.7 gives the output of the system given by equation (5.32) for a reduced value of the surface parameter. In this case the surface parameter is taken to be $C_{s}=\left[\begin{array}{ll}2 & 1\end{array}\right]$. The control law will be,

$$
u_{s}=\left[\begin{array}{ll}
-0.3 & -17.4996
\end{array}\right] x_{s}-5.4377 \mathrm{sat}\left(\frac{S_{s}}{0.1}\right)
$$

This example demonstrates clearly the need for an advanced stability analysis method to find the maximum value of the perturbed parameter that will ensure a stable closed loop system. Furthermore, a technique for surface design that will attenuate the excitation from the fast subsystem is needed. 


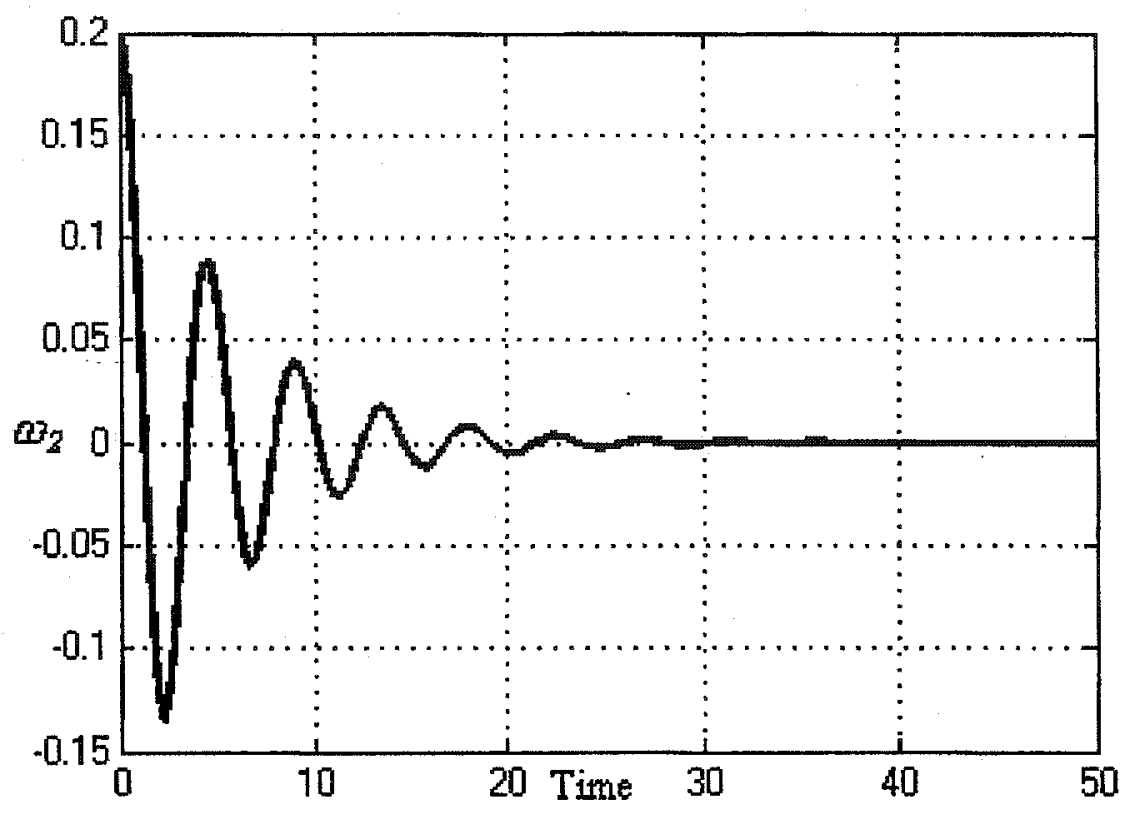

Figure 5.7.a: Full order closed loop time response for a single Sliding Mode Controller with a boundary layer with a different slope (Slow State)

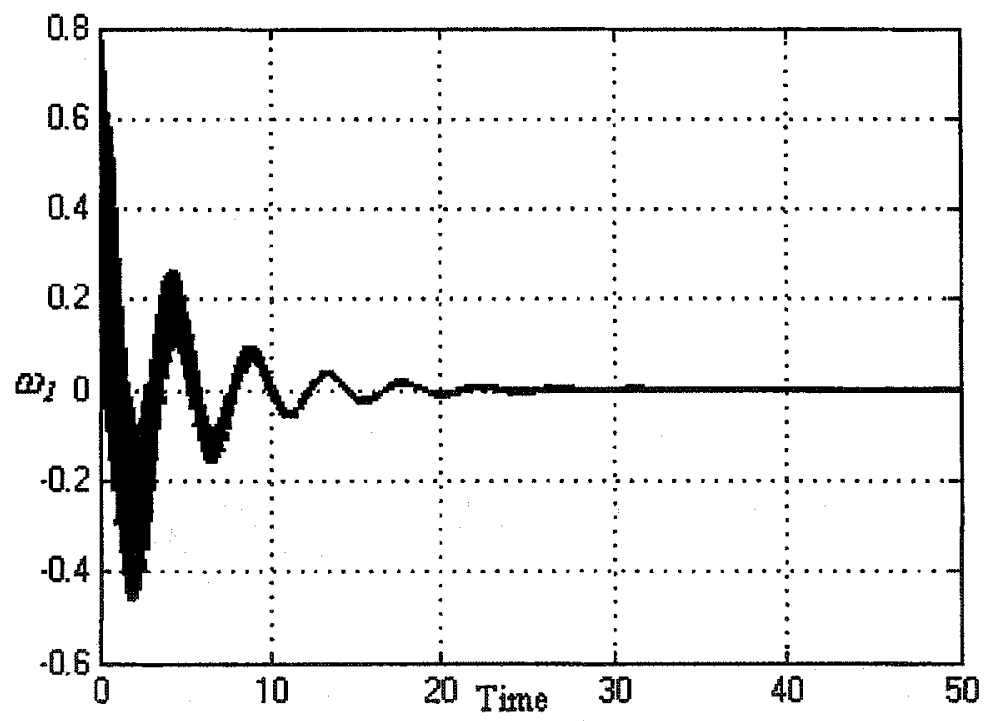

Figure 5.7.b: Full order closed loop time response for a single Sliding Mode Controller with a boundary layer with a different slope (Fast State)

The diagonalization method as shown in this section can avoid the error that is caused by approximation resulting from the quasi steady state method. The error due to this approximation can be significant if $\varepsilon$ is not small enough. However, the diagonalization method is not enough to ensure the stability of the system. In example 5.2 we have shown 
that when the full order model is controlled by a single sliding mode controller this can result in instability, both diagonalization and quasi steady state methods have the same unstable result as shown in Figure 5.2. This leads us to investigate the other two important factors, which are stability bounds and sliding surface design for our proposed controller.

\subsection{Stability analysis}

The slow sliding mode controller is designed using the slow reduced order system given by equation [30],

$\dot{x}_{s}=A_{0} x_{s}+B_{0} u_{s}$

The difference in this section from the previous methods for sliding mode control design, which are the equivalent control method and the tracking control method described in section 2.2 and 2.6 respectively, is that we will use the reaching law approach introduced by Gao [19] and explained in section (2.3). We define the linear switching surface as $S_{s}=C_{s} x_{s}$ and the dynamics during sliding are given by,

$\dot{x}_{s}=\left[I-B_{0}\left(C_{s} B_{0}\right)^{-1} C_{s}\right] A_{0} x_{s}=A_{e q} x_{s}$

To guarantee the local stability for this controller we must choose $C_{s}$ to ensure that the $(n-r)$ eigenvalues of the equivalent system given by equation (5.31) have negative real parts.

To study the stability of the closed loop full order system we will use the reaching control law [19] with continuous approximation [3] as shown in chapter 2 section (2.3). The dynamics of the slow subsystem when it is outside the sliding manifold are given by 
$\dot{S}_{s}=C_{s}\left(A_{0} x_{s}+B_{0} u_{s}\right)=-K S_{s}-Q \operatorname{sgn}\left(S_{s}\right)$

where $Q$ and $K$ are diagonal matrices with positive elements.

Based on Burton's [3] approach we replace the "sgn" function by the vector function

$\theta_{i}(s)=\frac{s_{i}}{\|S\|+\delta_{i}} \quad i=1,2, \ldots m$

where $\delta$ is a small positive constant and $S=\left[s_{1}, \ldots \ldots, s_{m}\right]^{T}$. Thus from equation (5.32) the control law has the form,

$u_{s}\left(x_{s}\right)=-\left(C_{s} B_{0}\right)^{-1}\left[K S_{s}+Q \theta\left(S_{s}\right)+C_{s} A_{o} x_{s}\right]$

The closed loop dynamics of the slow subsystem given by equation (5.30) can be written as,

$\dot{\boldsymbol{x}}=\left[I-B_{0}\left(C_{s} B_{0}\right)^{-1} C_{s}\right] A_{0} x_{s}-B_{o}\left(C_{s} B_{0}\right)^{-1}\left[K C_{s} x_{s}+Q \theta\left(S_{s}\right)\right]$

As discussed in chapter 3 a realizable controller requires that the system states $\boldsymbol{x}_{s}$ and $x_{f}$ be expressed in terms of the actual system states $x_{1}$ and $x_{2}$. This can be achieved by replacing $x_{s}$ by $x$ and $x_{f}$ by $z-z_{s}$, thus equation (5.34) can be rewritten as,

$u_{s}(x)=-\left(C_{s} B_{0}\right)^{-1}\left[K S_{s}+Q \theta\left(S_{s}\right)+C_{s} A_{0} x\right]$

where $S_{s}=C_{s} x$

\subsubsection{Stability analysis for the full order model with single sliding mode controller}

In this section the stability of the full order closed loop system under the sliding mode controller designed for the slow reduced order subsystem is addressed. We use Kokotovic's approach [30] for stability analysis, which is discussed in detail in chapter 3. 
Assume that the fast subsystem is stable and we design a controller based on the slow reduced order model such that (5.32) is satisfied. Thus the stability of the closed loop full order model can be proven for a certain interval of the perturbed parameter $\varepsilon$.

\section{Theorem 5.1}

Given that the fast subsystem is asymptotically stable (i.e $\operatorname{Re} \lambda\left(A_{22}\right)<0$ ) and the control, $u_{s}(x)=-\left(C_{s} B_{0}\right)^{-1}\left[K S_{s}+Q \theta\left(S_{s}\right)+C_{s} A_{0} x\right]$

is applied to the system given by equations (5.1) and (5.2) then there exists an $\varepsilon^{*}>0$ such that the closed loop system is asymptotically stable for all $\varepsilon \in\left(0, \varepsilon^{*}\right)$.

\section{Proof}

Define the singularly perturbed system given by (5.1) and (5.2) as,

$$
\begin{aligned}
& \dot{x}=f(x, z)=A_{11} x+A_{12} z+B_{1} u \\
& \varepsilon \dot{z}=g(x, z)=A_{21} x+A_{22} z+B_{2} u
\end{aligned}
$$

To prove this theorem using the Lyapunov approach we must have functions $V(x, z)$ and $W(x, z)$ satisfying the following equations respectively [30],

$$
\begin{aligned}
& \frac{\partial V}{\partial x} f(x, z) \leq-\alpha_{1} \psi^{2}(x) \quad \alpha_{1}>0 \\
& \frac{\partial W}{\partial z} g(x, z) \leq-\alpha_{2} \phi^{2}\left(z-z_{s}\right) \quad \alpha_{2}>0
\end{aligned}
$$

and to find the maximum value of $\varepsilon$ we must also satisfy the interconnection conditions described in chapter 3 and given by,

$$
\frac{\partial V}{\partial x}\left[f(x, z)-f\left(x, z_{s}\right)\right] \leq \beta_{l} \psi(x) \phi\left(z-z_{s}\right)
$$


$\frac{\partial W}{\partial x} f(x, z) \leq \gamma \phi^{2}\left(z-z_{s}\right)+\beta_{2} \psi(x) \phi\left(z-z_{s}\right)$

where $\phi($.$) and \psi($.$) are scalar functions of vector arguments which vanish only when$ their arguments are zero and $z_{s}$ is the slow part of vector $z$ as has been described in chapter 3 and is given by,

$$
z_{s}=h(x)=-A_{22}^{-1}\left(A_{2 I} x+B_{2} u_{s}\right)
$$

We first define the Lyapunov function $V(x, z)$ for the slow subsystem given by equation (5.30). We choose $V(x, z)$ as [25] and [26],

$V(x, z)=\frac{1}{2} x^{T} U^{-T} U^{-1} x$

where $U$ in this case is the matrix of the eigenvectors of the projector $F_{s}=\left[I-B_{0}\left(C_{s} B_{0}\right)^{-1} C_{s}\right]$

Based on this choice, Innocenti [25][26] proves that the condition given by equation (5.39) can be satisfied as,

$\frac{\partial V}{\partial x} \dot{x} \leq-\alpha_{I}\|x\|^{2}$

where $\alpha_{1} \in R_{+}$given by $\alpha_{l}=\lambda_{\min }(M)\left\|U^{-1}\right\|^{2}$ and $M=\left[\begin{array}{cc}K & 0 \\ 0 & \lambda_{e q}\end{array}\right]$ where $\lambda_{e q}$ is a diagonal matrix of the nonzero eigenvalues of $A_{e q}$ defined in equation (5.31). At this point the proposed method diverges from the proof offered by AlvarezGallegos [1] and Innocenti [25][26]. They use two sliding mode controllers to control the full order singularly perturbed system. The choice of the Lyapunov function for the fast subsystem in [25] is given by 
$W(x, z)=\frac{1}{2}\left(z-z_{s}\right)^{T} U_{f}^{-T} U_{f}^{-1}\left(z-z_{s}\right)$

where $U_{f}$ is a matrix whose columns are the eigenvectors of $F_{f}$ given by, $F_{f}=\left[I-B_{2}\left(C_{f} B_{2}\right)^{-1} C_{f}\right]$. For single sliding mode controller there is no such projection operation in the fast channel. Consequently, $U_{f}$ does not exist and the choice of Lyapunov function for the fast subsystem in [26] given by equation (5.46) is not appropriate for our controller. Therefore, we define a new Lyapunov function for the fast subsystem because we are using a sliding mode controller based on the slow state only. We need to satisfy the second condition given by equations $(5.40)$ and (5.42) for the fast subsystem,

$$
g(x, z)=A_{21} x+A_{22} z+B_{2}\left(u_{s}+u_{f}\right)
$$

Adding and subtracting the steady state value of $z$, given by $z_{s}$, the above equation can be rewritten as,

$$
g(x, z)=A_{21} x+A_{22}\left(z-z_{s}\right)+A_{22} z_{s}+B_{2}\left(u_{s}\right)
$$

Substituting the value of $z_{s}=-A_{22}^{-1}\left(A_{21} x+B_{2} u_{s}\right)$ in equation (5.48) gives,

$$
g(x, z)=A_{22}\left(z-z_{s}\right)
$$

As a result we introduce the Lyapunov function $W(x, z)$ as,

$$
W(x, z)=\frac{1}{2}(z-h(x))^{T} P(z-h(x))=\frac{1}{2}\left(z-z_{s}\right)^{T} P\left(z-z_{s}\right)
$$

where $P$ satisfies the Lyapunov equation given by

$$
A_{22}^{T} P+P A_{22}=-I_{m}
$$


Given equation (5.49) and equation (5.50) and taking into account the assumption that the fast subsystem is stable, one can prove the following inequality,

$$
\frac{\partial W}{\partial z} g(x, z) \leq-\frac{1}{2}\left\|z-z_{s}\right\|^{2}
$$

Equation (5.52) satisfies condition (5.40) with $\alpha_{2}=\frac{1}{2}$ and $\phi\left(z-z_{s}\right)=\left\|z-z_{s}\right\|$

The second step in the stability analysis is to satisfy the interconnection condition given by equation (5.41) and (5.42). Differentiating $\boldsymbol{W}$ with respect to $\boldsymbol{x}$ gives,

$$
\frac{\partial W}{\partial x}=-\left(z-z_{s}\right)^{T} P \frac{\partial z_{s}}{\partial x}
$$

Recall from equation (5.43) where $z_{s}=-A_{22}^{-1}\left(A_{21} x+B_{2} u_{s}\right)$ therefore,

$$
\frac{\partial z_{s}}{\partial x}=-A_{22}^{-1}\left[A_{21}-B_{2}\left(C_{s} B_{0}\right)^{-1}\left(K C_{s}+Q \frac{\partial \theta\left(C_{s} x\right)}{\partial x}+C_{s} A_{0}\right)\right]
$$

Equation (5.54) can be rewritten as

$$
\frac{\partial z_{s}}{\partial x}=G_{1}+G_{2} \frac{\partial \theta\left(C_{s} x\right)}{\partial x}
$$

Where

$$
G_{1}=-A_{22}^{-1}\left[A_{21}-B_{2}\left(C_{s} B_{0}\right)^{-1}\left(K_{s} C_{s}+C_{s} A_{0}\right)\right] \text { and } G_{2}=A_{22}^{-1} B_{2}\left(C_{s} B_{0}\right)^{-1} Q
$$

Recall the function $f(x, z)$ is given by,

$f(x, z)=A_{11} x+A_{12} z+B_{1} u$.

Add and subtract $z_{s}$ and set $u=u_{s}$ since $u_{f}=0$ then equation (5.56) can be written as,

$f(x, z)=A_{11} x+A_{12}\left(z-z_{s}\right)+A_{I 2} z_{s}+B_{1} u_{s}$

Substituting the value of $z_{s}=-A_{22}^{-1}\left(A_{21} x+B_{2} u_{s}\right)$ in the above equation thus,

$$
f(x, z)=A_{0} x+B_{0} u_{s}+A_{12}\left(z-z_{s}\right)
$$


where $A_{0}=A_{11}-A_{12} A_{22}^{-1} A_{21}$ and $B_{0}=B_{1}-A_{12} A_{22}^{-1} B_{2}$

Substituting the value of $u_{s}$ given by equation (5.34) in equation (5.58) gives,

$f(x, z)=\left[A_{0} x-B_{0}\left(C_{s} B_{0}\right)^{-1}\left(K_{s} C_{s} x+Q \theta\left(S_{s}\right)+C_{s} A_{0} x\right)\right]+A_{12}\left(z-z_{s}\right)$

Thus equation (5.59) can be rewritten as,

$f(x, z)=G_{3} x+G_{4} \theta\left(S_{s}\right)+A_{12}\left(z-z_{s}\right)$

where

$G_{3}=\left[I-B_{0}\left(C_{s} B_{0}\right)^{-1} C_{s}\right] A_{0}-B_{0}\left(C_{s} B_{0}\right)^{-1} K C_{s}$

$G_{4}=-B_{0}\left(C_{s} B_{0}\right)^{-1} Q$

From equation (5.60) and equation (5.53) condition (5.42) can be satisfied as follows,

$\frac{\partial W}{\partial x} f(x, z)=\left[-\left(z-z_{s}\right)^{T} P\left(G_{1}+G_{2} \frac{\partial \theta\left(S_{s}\right)}{\partial x}\right)\right]\left[G_{3} x+G_{4} \theta\left(S_{s}\right)+A_{12}\left(z-z_{s}\right)\right]$

Thus taking the norm of the right hand side terms of the above equation gives,

$$
\begin{aligned}
& \frac{\partial W}{\partial x} f(x, z) \leq\left\|-P G_{1} G_{3}\right\|\left\|\left(z-z_{s}\right)\right\|\|x\| \\
& +\left\|\left(z-z_{s}\right)\right\|\left\|P G_{1} G_{4}\right\|\left\|\theta\left(S_{s}\right)\right\| \\
& +\left\|\left(z-z_{s}\right)\right\|^{2}\left\|-P G_{1} A_{12}\right\| \\
& \quad+\left\|\left(z-z_{s}\right)\right\|\left\|-P G_{2}\right\|\left\|\frac{\partial \theta\left(S_{s}\right)}{\partial x}\right\|\left\|G_{3}\right\|\|x\| \\
& +\left\|\left(z-z_{s}\right)\right\|\left\|-P G_{2}\right\|\left\|\frac{\partial \theta\left(S_{s}\right)}{\partial x}\right\|\left\|G_{4}\right\|\left\|\theta\left(S_{s}\right)\right\| \\
& \quad+\left\|\left(z-z_{s}\right)\right\|^{2}\left\|-P G_{2}\right\|\left\|\frac{\partial \theta\left(S_{s}\right)}{\partial x}\right\|\left\|A_{12}\right\|
\end{aligned}
$$

But based on continuous approximation analysis offered by Burton [3], $\theta\left(S_{s}\right)$ can be written as [25],

$\theta\left(S_{s}\right) \leq\left\|\Omega C_{s}\right\|\|x\|$ and 


\section{$\left\|\frac{\partial \theta\left(S_{s}\right)}{\partial x}\right\| \leq\|\Omega\|\left\|C_{s}\right\|$}

where $\Omega=\operatorname{diag}\left\{\frac{1}{\delta_{i}}\right\}$

This satisfies condition (5.42) and equation (5.61) can be rewritten as

$\frac{\partial W}{\partial x} f(x, z) \leq \gamma\left\|z-z_{s}\right\|^{2}+\beta_{2}\|x\|\left\|z-z_{s}\right\|$

where

$\gamma=\left\|-P G_{1} A_{12}\right\|+\left\|-P G_{2}\right\|\|\Omega\|\left\|C_{s}\right\|\left\|A_{12}\right\|$ and

$\beta_{2}=\left\|-P G_{I} G_{3}\right\|+\left\|-P G_{1} G_{4}\right\|\left\|\Omega C_{s}\right\|$

$+\left\|-P G_{2}\right\|\|\Omega\|\left\|C_{s}\right\|\left\|G_{3}\right\|+\left\|-P G_{2}\right\|\|\Omega\|\left\|C_{s}\right\|\left\|G_{4}\right\|\left\|\Omega C_{s}\right\|$

The second interconnection condition given by equation (5.41) can also be analyzed and proven given that,

$f(x, z)=A_{0} x+A_{12}\left(z-z_{s}\right)+B_{0} u_{s}$

and

$f\left(x, z_{s}\right)=A_{0} x+B_{0} u_{s}$

Differentiating $V$ given by equation (5.44) with respect to $x$ gives,

$\frac{\partial V}{\partial x}=x^{T} U^{-T} U^{-1}=x^{T} J$

where $J=U^{-T} U^{-I}$. Subtracting equation (5.64) from equation (5.63) and multiplying by equation (5.65) gives us,

$$
\frac{\partial V}{\partial x}\left[f\left(x, z_{0}\right)-f\left(x, z_{s}\right)\right]=x^{T} J A_{12}\left(z-z_{s}\right)
$$

Taking the norm of the right hand side of equation (5.66) gives us the inequality, 


$$
\frac{\partial V}{\partial x}\left[f(x, z)-f\left(x, z_{s}\right)\right] \leq\left\|J A_{12}\right\|\|x\|\left\|\left(z-z_{s}\right)\right\|
$$

This satisfies condition (5.41) given that $\beta_{1}=\left\|J A_{12}\right\|$ which completes the proof of theorem (5.1). The maximum value of the perturbed parameter can be given by [30],

$$
\varepsilon^{*}=\frac{\alpha_{1} \alpha_{2}}{\alpha_{1} \gamma+\beta_{1} \beta_{2}}
$$

This result proves that the single sliding mode controller is able to make the full order system globally stable $\forall \varepsilon \in\left(0, \varepsilon^{*}\right)$

\subsubsection{Illustrative example}

\section{Example 5.3}

Consider a magnetic tape control system given by

$$
\begin{aligned}
& {\left[\begin{array}{l}
\dot{x}_{1} \\
\dot{x}_{2} \\
\varepsilon \dot{z}_{1} \\
\varepsilon \dot{z}_{2}
\end{array}\right]=\left[\begin{array}{cccc}
0 & 0.4 & 0 & 0 \\
0 & 0 & 0.345 & 0 \\
0 & -0.524 & -0.465 & 0.262 \\
0 & 0 & 0 & -1
\end{array}\right]\left[\begin{array}{l}
x_{1} \\
x_{2} \\
z_{1} \\
z_{2}
\end{array}\right]+\left[\begin{array}{l}
0 \\
0 \\
0 \\
1
\end{array}\right] u} \\
& y=\left[\begin{array}{llll}
1 & 0 & 0 & 0 \\
0 & 0 & 1 & 0
\end{array}\right]\left[\begin{array}{l}
x_{1} \\
x_{2} \\
z_{1} \\
z_{2}
\end{array}\right] .
\end{aligned}
$$

This can be put in standard singularly perturbed form as,

$$
\begin{aligned}
& \dot{x}=A_{11} x+A_{12} z+B_{1} u, \\
& \varepsilon \dot{z}=A_{12} x+A_{22} z+B_{2} u,
\end{aligned}
$$


where $A_{l l}=\left[\begin{array}{cc}0 & 0.4 \\ 0 & 0\end{array}\right], A_{12}=\left[\begin{array}{cc}0 & 0 \\ 0.345 & 0\end{array}\right], A_{21}=\left[\begin{array}{cc}0 & -0.524 \\ 0 & 0\end{array}\right]$,

$A_{22}=\left[\begin{array}{cc}-0.465 & 0.262 \\ 0 & -1\end{array}\right] B_{1}=\left[\begin{array}{l}0 \\ 0\end{array}\right]$ and $B_{2}=\left[\begin{array}{l}0 \\ 1\end{array}\right]$

Setting $\varepsilon=0$ in equation (5.70) gives us the reduced slow subsystem

$\dot{x_{s}}=A_{0} x_{s}+B_{0} u$

where $A_{0}=A_{1 l}-A_{12} A_{22}^{-1} A_{2 I}=\left[\begin{array}{cc}0 & 0.4 \\ 0 & -0.38\end{array}\right]$ and $B_{0}=B_{I}-A_{12} A_{22}^{-I} B_{2}=\left[\begin{array}{c}0 \\ 0.1944\end{array}\right]$

The surface parameter for the sliding mode controller is chosen based on the equivalent system given by equation (5.5). Given the slow subsystem,

$\dot{x}_{s}=A_{0} \boldsymbol{x}_{s}+B_{0} u$

choose the surface parameters as $C_{s}=\left[\begin{array}{ll}C_{1} & C_{2}\end{array}\right]$ and apply this value to equation (5.5) and use the Matlab function "syms" to get,

$\dot{\boldsymbol{x}}_{\text {eqs }}=\left[I-B_{0}\left(C_{s} B_{0}\right)^{-1} C_{s}\right] A_{0} \boldsymbol{x}_{e q s}=\left[\begin{array}{cc}0 & 2 / 5 \\ 0 & -2 C_{1} / 5 C_{2}\end{array}\right] \boldsymbol{x}_{e q s}$

We will select the eigenvalues on the sliding surface to be, $\lambda_{1}=0$ and $\lambda_{2}=-0.6$ to obtain a time constant for the slow subsystem in the sliding mode to be $T_{s} \cong 1.6$ hence the corresponding surface parameters are $C_{s}=\left[\begin{array}{ll}1.5 & 1\end{array}\right]$ and we set $K=1, Q=1$ and $\delta=0.2$. Substituting into equation (5.36) the control law will be,

$u_{s}=\left[\begin{array}{ll}0 & 1.0866\end{array}\right] x_{s}-5.144\left[S_{s}+\theta\left(S_{s}\right)\right]$

Based on the analysis given by equations (5.44)-(5.68) we find the value of $\alpha_{1}=3.05$, $\alpha_{2}=0.5, \beta_{1}=0.6281, \beta_{2}=346.616$ and $\gamma=10.7225$ thus the maximum value of the 
perturbation parameter is $\varepsilon^{*} \cong 0.006$. We have to stress here that this value is sufficient but not necessary.

Figure 5.8 illustrates the closed loop time response of the full order system. It is observed that the closed loop system shows good performance. In fact, the states of the full order model $(x, z)$ converge to the equilibrium point even with changes on the perturbation parameter $\varepsilon$, which indicates good properties for our controller. Figure 5.9 and 5.10 show closed loop response for different values of $\varepsilon$. In Figure 5.11 we see that further increase in the value of $\varepsilon$ leads to an unstable system. Figure 5.12 illustrates the relationship between the maximum perturbed parameter $\varepsilon^{*}$ and slope of sliding surface given that $K=0$ and $Q=1$. Choosing $K=0$ will reduce the reaching control law given by equation (5.36) to be similar to the equivalent control law given by equation (5.11)

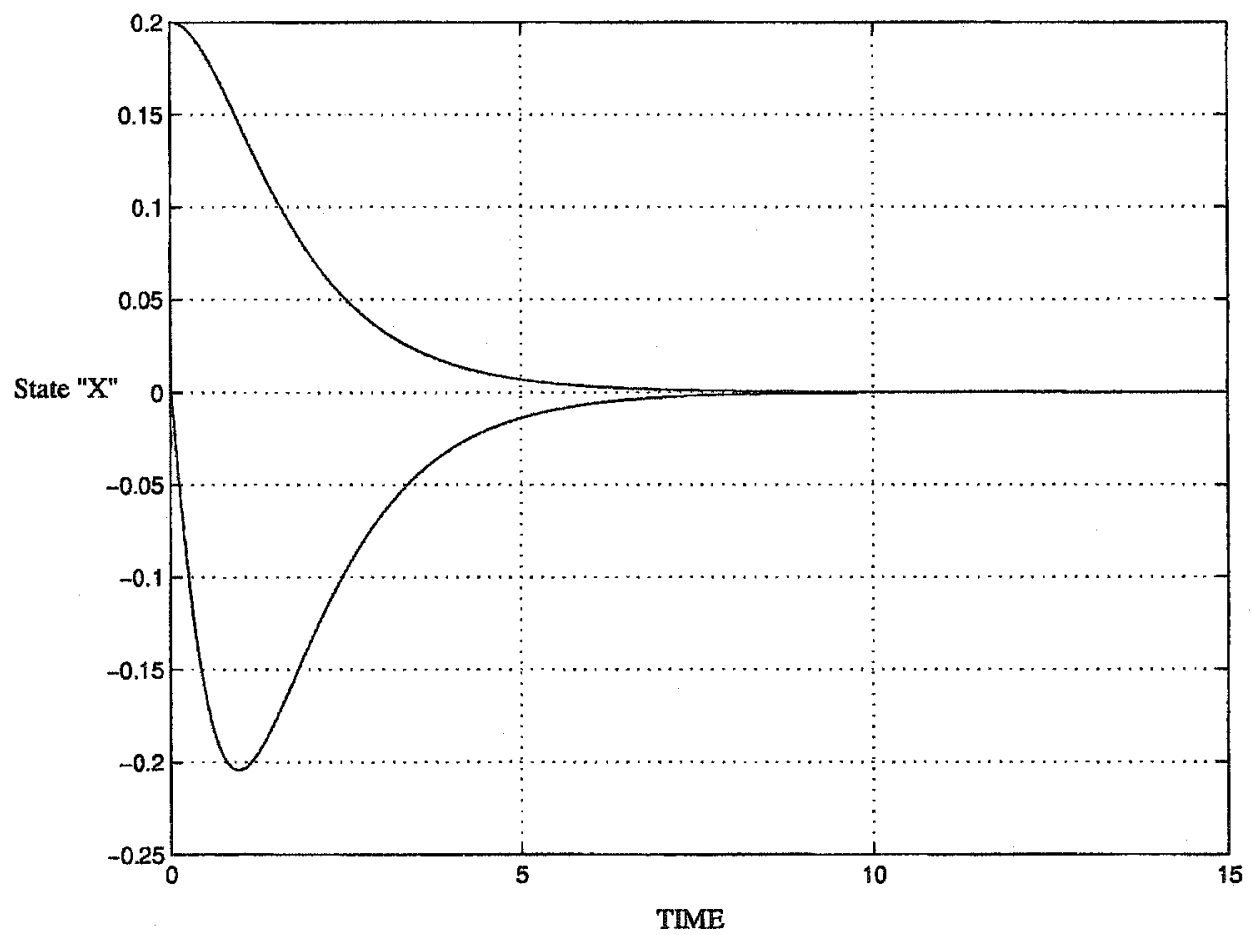

Figure 5.8.a: Full order closed loop time response for sliding mode control $\varepsilon=0.005$ (slow state) 


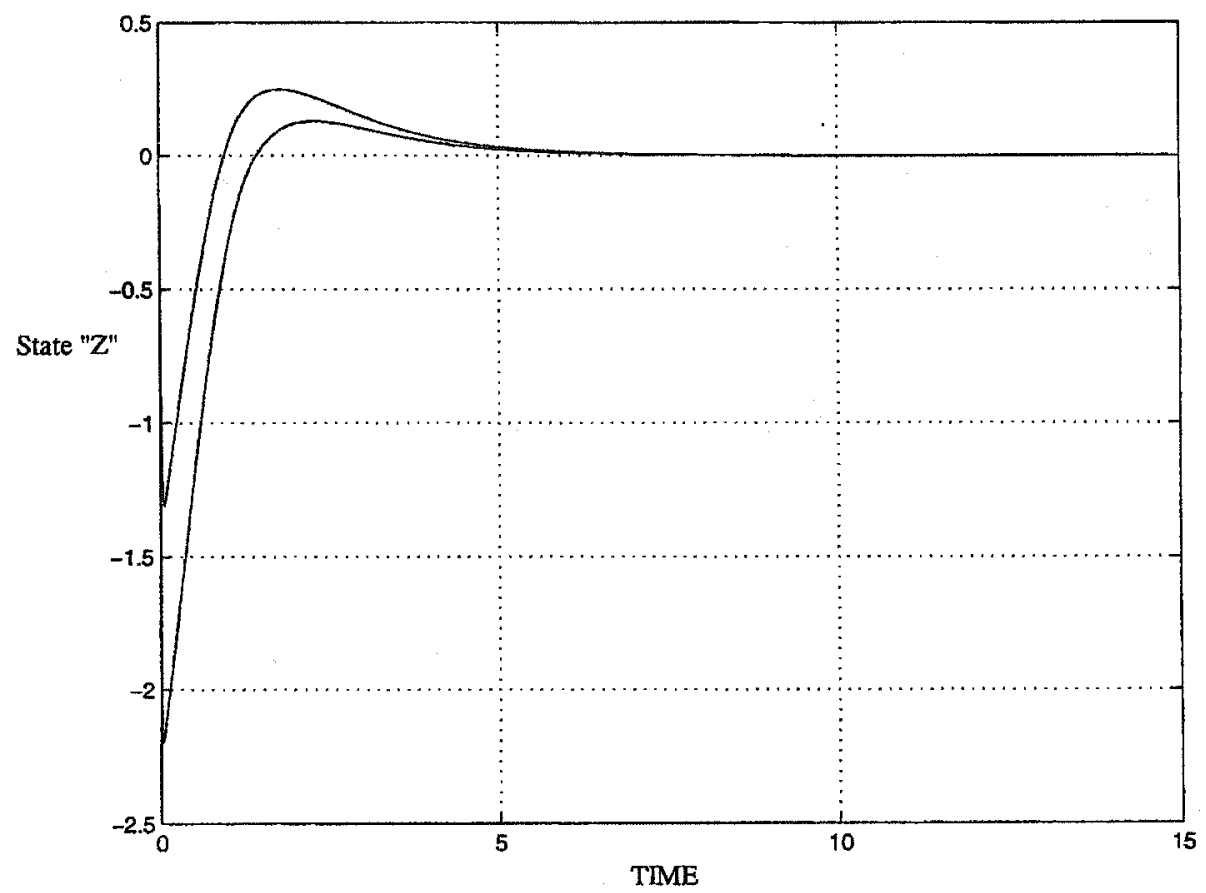

Figure 5.8.b: Full order closed loop time response for sliding mode control $\varepsilon=0.005$ (fast state)

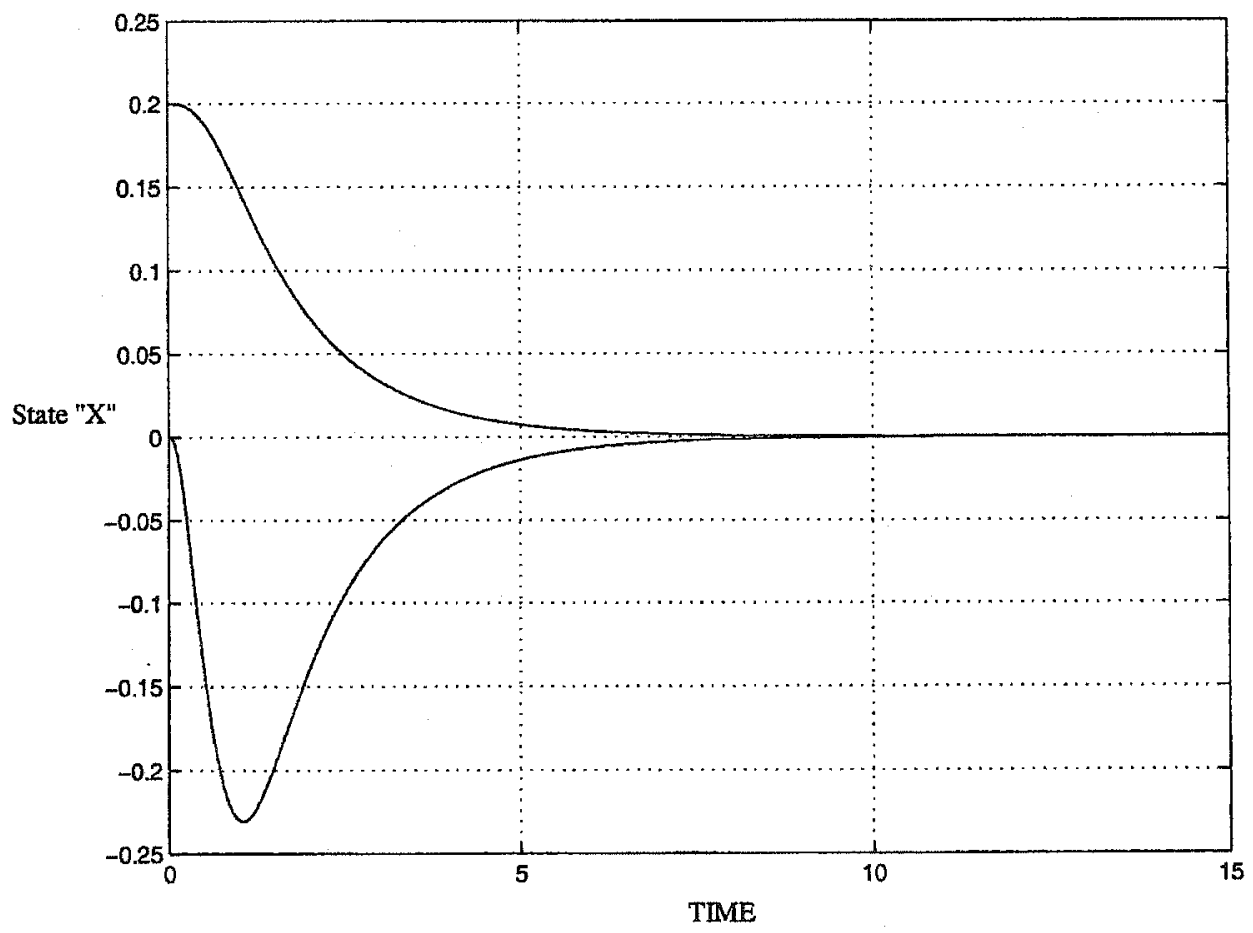

Figure 5.9.a: Full order closed loop time response for sliding mode control $\varepsilon=0.05$ (slow state) 


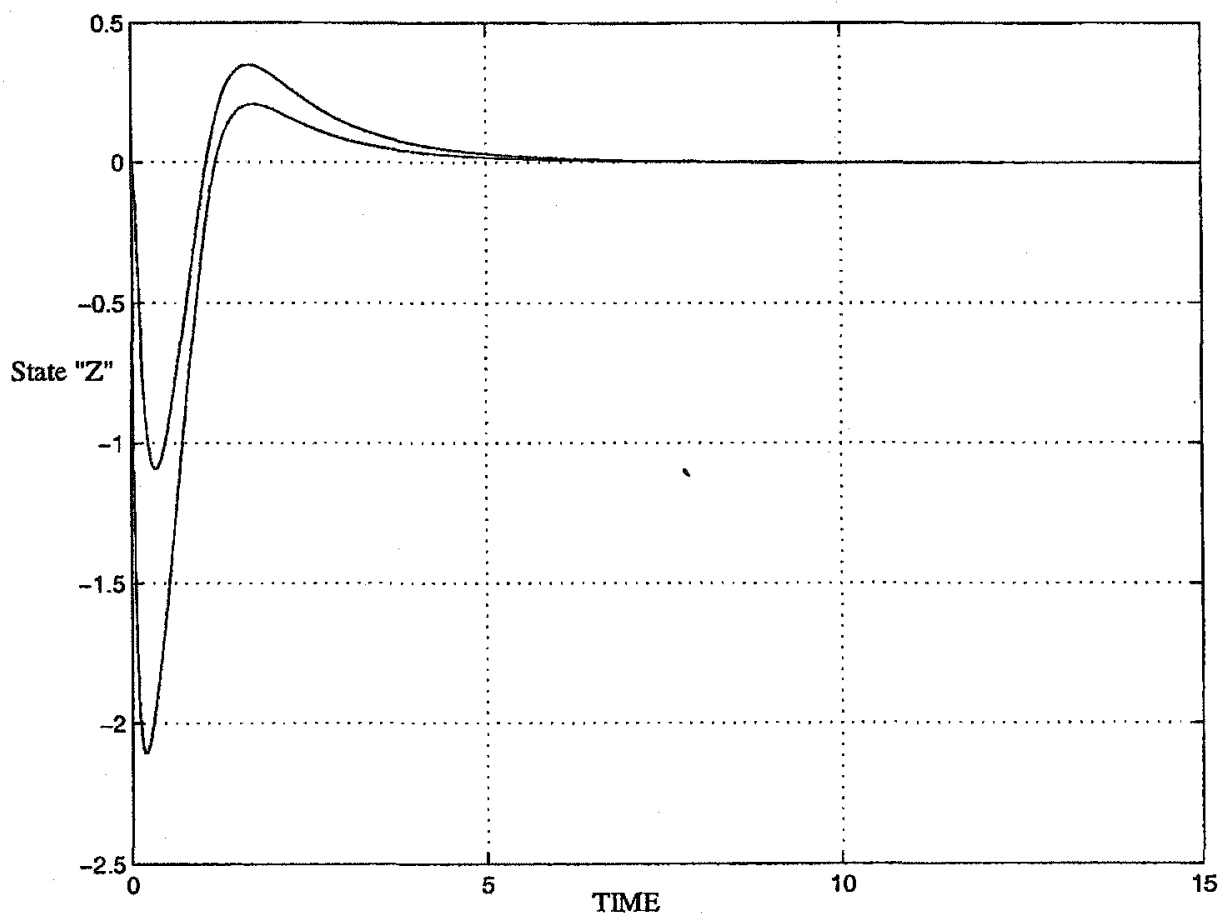

Figure 5.9.b: Full order closed loop time response for sliding mode control $\varepsilon=0.05$ (fast state)

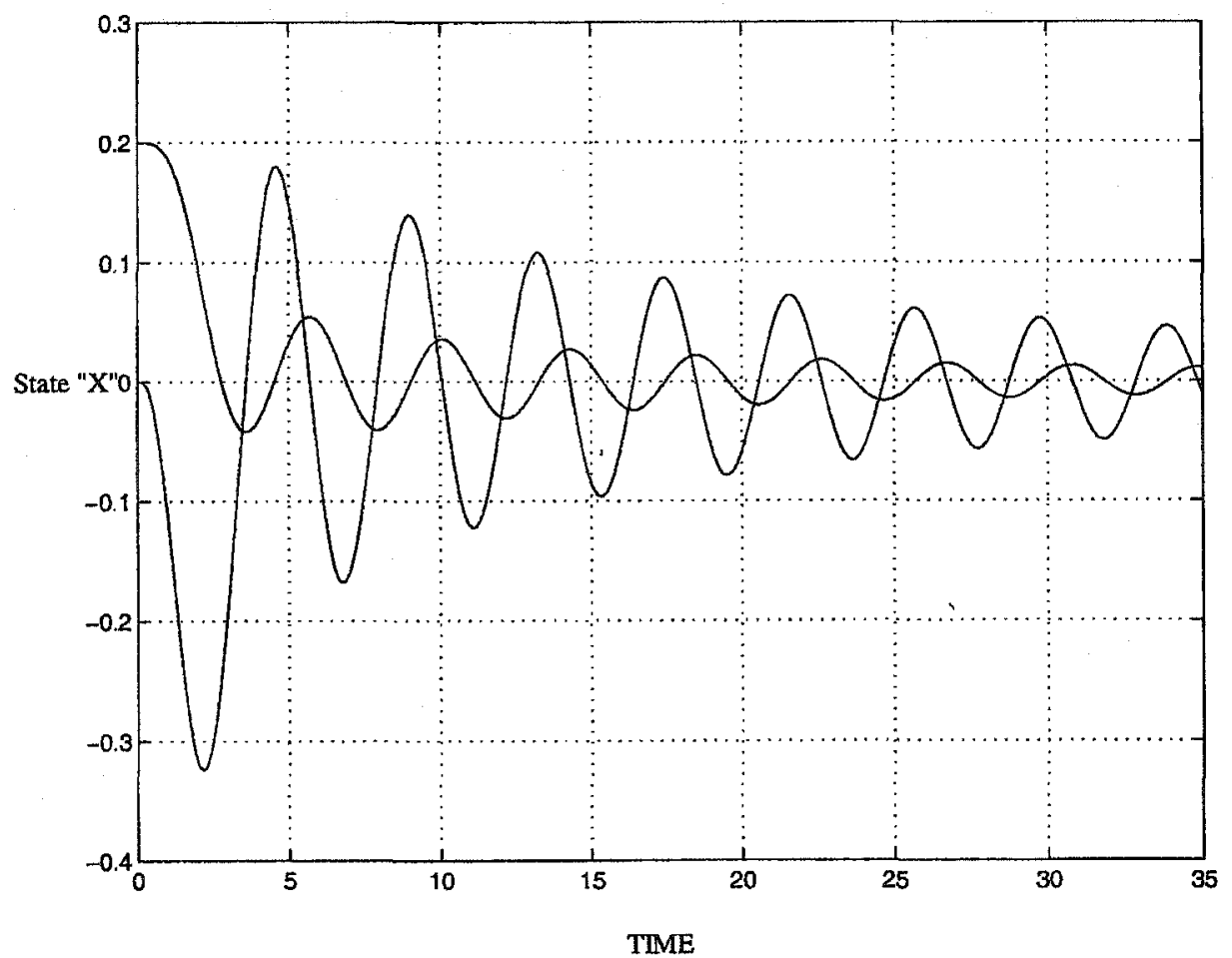

Figure 5.10.a: Full order closed loop time response for sliding mode control $\varepsilon=0.3$ ( slow state) 


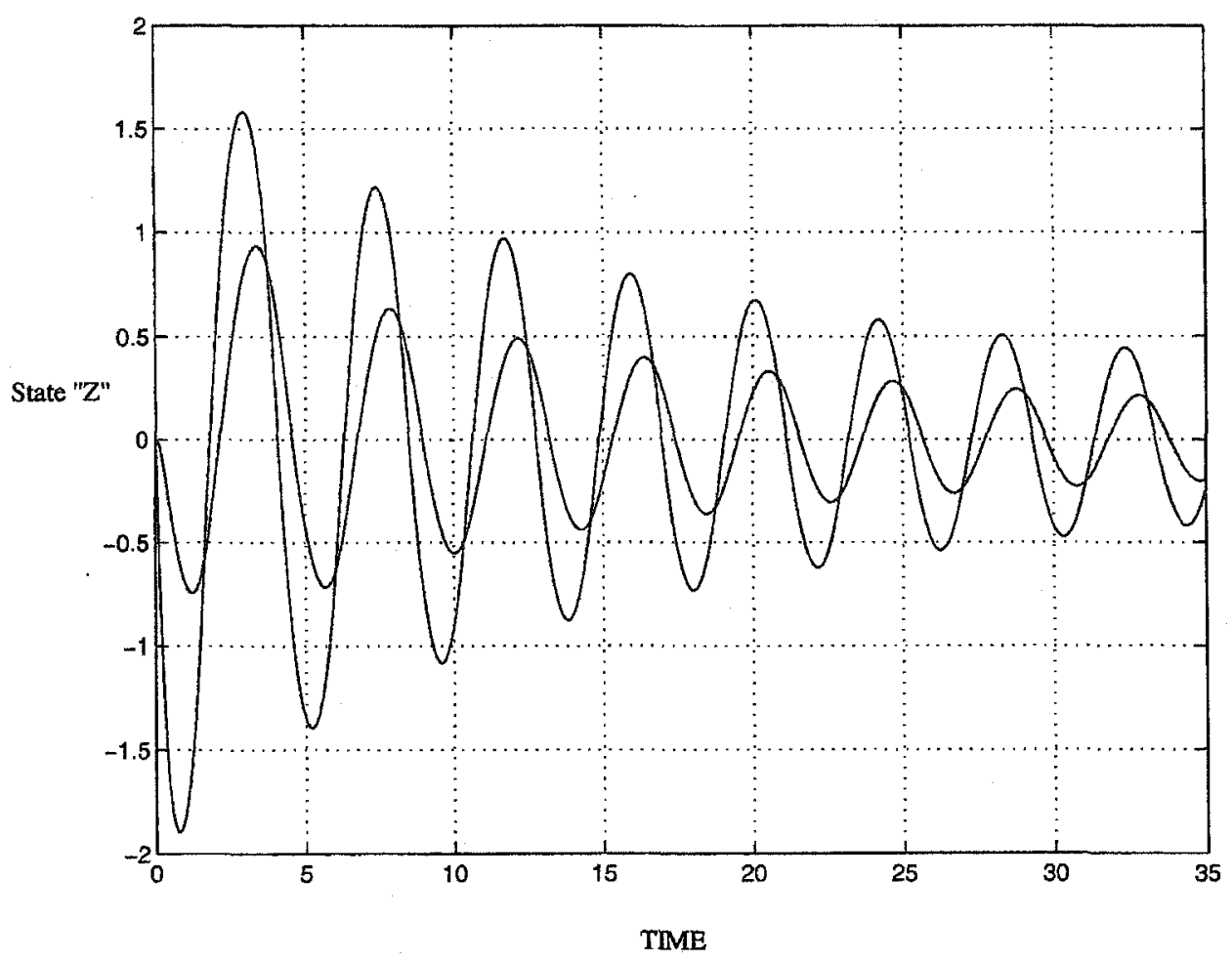

Figure 5.10.b: Full order closed loop time response for sliding mode control $\varepsilon=0.3$ (fast state)

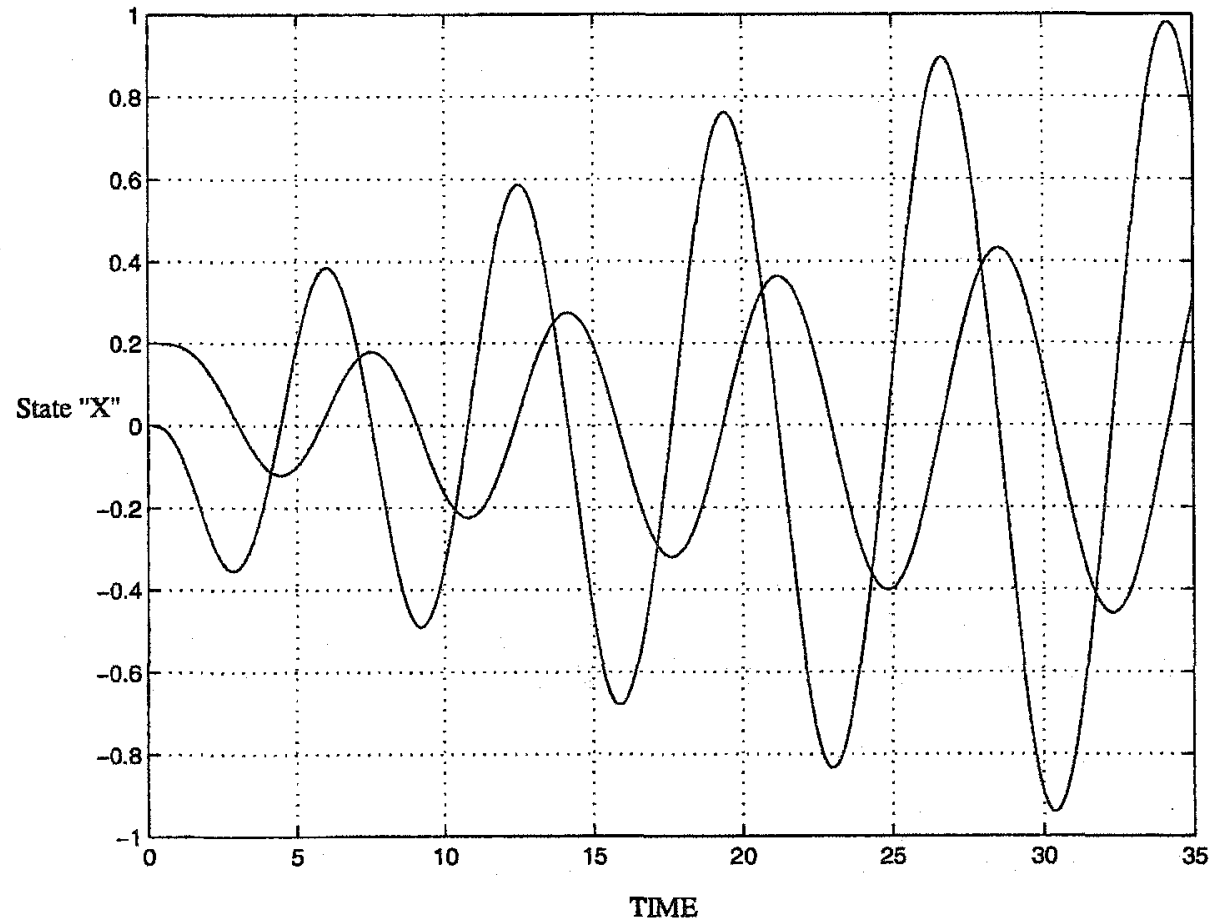

Figure 5.11.a: Full order closed loop time response for sliding mode control $\varepsilon=0.5$ ( slow state) 


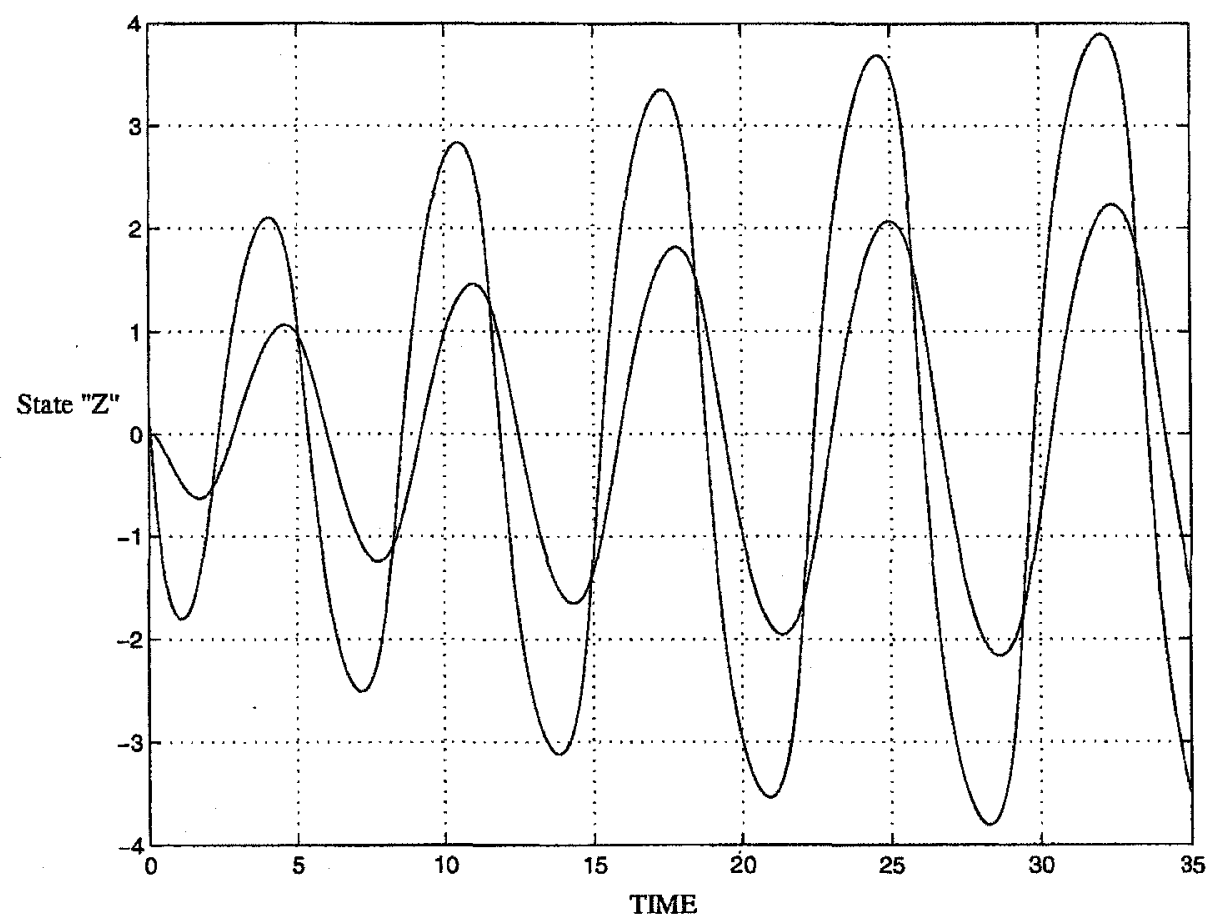

Figure 5.11.b: Full order closed loop time response for sliding mode control $\varepsilon=0.5$ (fast state)

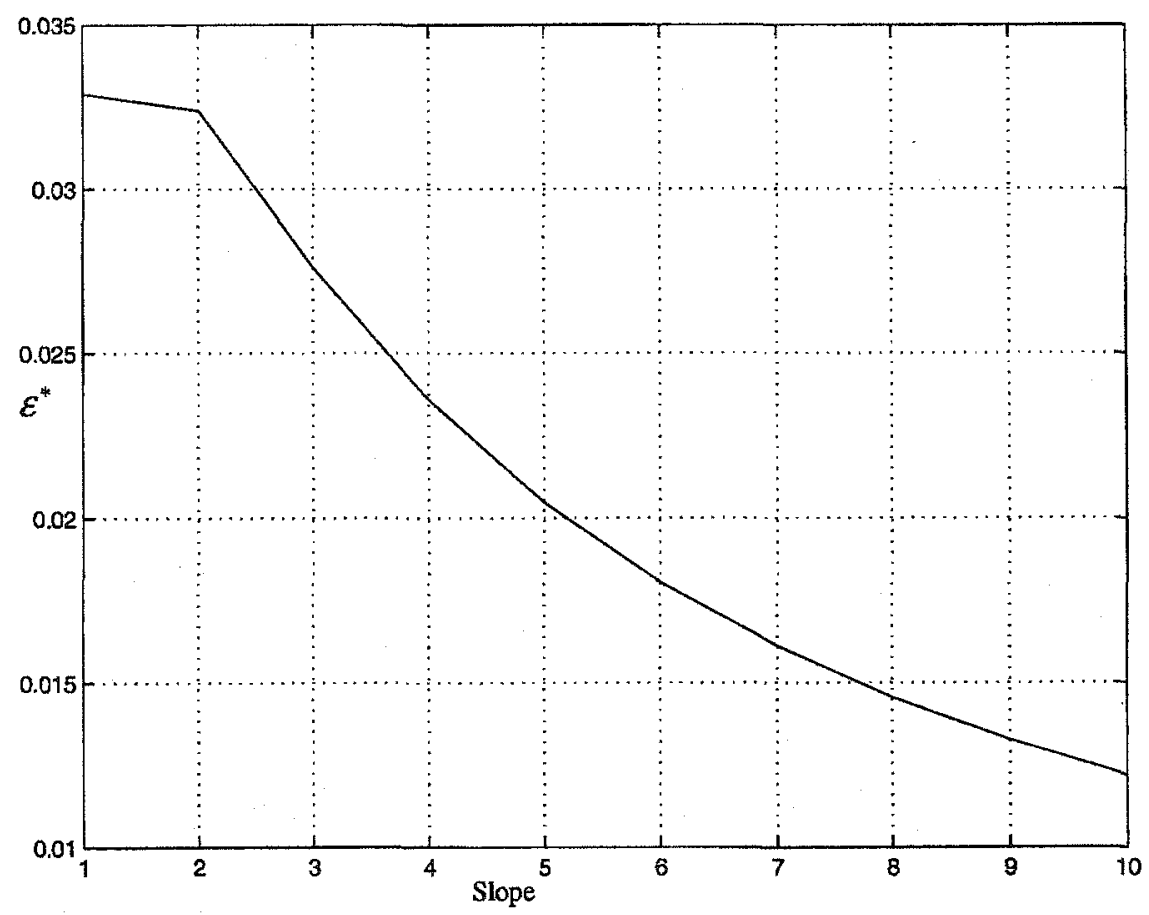

Figure 5.12: The maximum perturbation parameter versus the slope of sliding surface 
In this chapter we proposed using a single sliding mode controller for the full order singularly perturbed system. The design method that we use is different than what has been proposed in the literature [22][57]. The sliding surface in our proposed controller has been design based on an iterative design process where the lowest frequency of the fast mode is taken to be the starting point for the iterative process. The design of the feedback gain and the switching gain are based on the slow subsystem. The main advantage of the proposed controller is that for the first time we take into consideration the effect of the high frequency modes when simulating the response of the closed loop system with a sliding mode controller as shown in examples 5.1, 5.2 and 5.3. We show that a single sliding mode controller can be used to control the full order singularly perturbed system. Consequently for a certain desired performance we use low control efforts. This is one of the advantages of the proposed controller. Furthermore the control law for the proposed controller given by equation (5.34) contains only the slow states, which means that there is no need to measure the fast state, which is usually difficult to measure.

In this chapter, we have compared the proposed diagonalization method with the quasi steady state method that is proposed in the literature [12][22][50]. The advantage of using the diagonalization method is that we can obtain a more accurate performance in comparison to the quasi steady state method. This is shown in Figure 5.1. However designing a single sliding mode controller for the slow subsystem obtained using the diagonalization method means that we have to measure the fast states. This is a disadvantage and therefore the quasi steady state method is used to design the single sliding mode controller in the next sections. 
In the last section of this chapter we discussed the stability of the closed loop system when using a single sliding mode controller for the full order singularly perturbed system. It was shown in Figure 5.2 and 5.5 that the selection of surface parameters results in an unstable response. The stability analysis uses the Lyapunov approach proposed by Kokotovic [30] and Innocenti [25]. The contribution in deriving the stability bounds is the choice of Lyapunov function proposed in equation (5.50) for the fast subsystem. This Lyapunov function satisfies the four conditions of stability. In this section, it has been shown that the value of the perturbation parameter $\varepsilon$ has an effect on the stability of the full order singularly perturbed model. When we design a single sliding mode controller for the full order singularly perturbed system, it becomes necessary to investigate the bounds of the maximum perturbation parameter $\varepsilon$ that will preserve stability of the closed loop model. We have shown from the sufficient theoretical condition the relation between $\varepsilon^{*}$ and the sliding surface as illustrated Figure 5.12. It has been demonstrated by simulation that the bound for the perturbation parameter is a conservative condition that is common with Lyapunov stability analysis. 


\section{Chapter 6 Sliding Surface Design in the}

\section{Frequency Domain}

\subsection{Introduction}

In this chapter, the frequency shaped single sliding mode controller for singularly perturbed systems is introduced. One of the three main problems for designing a single sliding mode controller for a full order singularly perturbed system, as described in chapter 5 , is the choice of surface parameters. It was shown in chapter 5 Figure 5.2 and Figure 5.5, that if the sliding surface is not correctly designed then instability could result. Frequency shaped sliding mode controllers offer a good solution for this problem. Furthermore, using frequency shaped sliding mode controllers for singularly perturbed systems allow us to introduce a second contribution to the field of frequency shaped sliding mode controllers. The contribution focuses on choosing the appropriate weighting function for the closed loop system. Thus there are two contributions in this chapter. The first is the use of a frequency shaped sliding mode controller for the singularly perturbed system. The second contribution is the appropriate choice of weighting function for the $H^{\infty}$ frequency shaped sliding mode controller.

An effective approach to robust control is sliding mode control, as has been discussed in chapter 2. The main characteristic of a sliding mode control system is the nonlinear control action, which forces the state trajectories of the system to reach the intersection of a desired set of sliding hyper-planes [54]. The state trajectories move along the sliding 
hyper planes until the origin is reached. As shown before, the advantage of the method is that the closed loop system can be designed to be robust with respect to certain classes of parametric uncertainty and external disturbances [15].

It is known that sliding mode controllers have two phases in the controller design: one is the reaching phase where the state moves from an arbitrary initial condition towards the sliding surface and the other is the sliding phase where the state follows the sliding surface. Choosing the sliding surface plays an important role in the response and stability of sliding mode control systems. It is desired that the surface parameters satisfy two conflicting objectives. One is that the system has a fast closed loop time response and the second is that the sliding mode controller does not excite the high frequency dynamics such that chattering does not occur and the system remains stable.

To solve this problem, an $H^{\infty}$ control technique has been used to obtain the appropriate sliding surface. It is important to state that there are two control approaches for sliding mode controller design. In the first approach [23][57], the algorithm is based on the static or conventional sliding hyper-plane. The sliding hyper-plane in this approach is designed by a linear quadratic regulator [LQ] control method. In the second approach [21][32][41][52][61], the main idea is based on a dynamic sliding hyper-plane which is defined in the augmented state space to account for frequency shaped weightings, through the frequency shaped formulation. The design method permits frequency shaping of the sliding mode dynamics through the introduction of compensator dynamics in the sliding mode. This class of sliding surface provides additional flexibility in developing control laws to attenuate the frequency contents of the sliding mode 
dynamics such that unmodeled dynamics are minimally excited. This can be done either using linear quadratic regulator, $H^{2}$ optimal control or $H^{\infty}$ optimal control

A method for designing the sliding surface of a variable structure control system using the frequency design advantages that come with $H^{\infty}$ is introduced by [41][52]. $H^{\infty}$ theory is a well-known control technique used to suppress high frequency modes of the controlled plant. This design method for sliding mode control has been proposed to satisfy two conflicting requirements: fast response and stability with regard to the unmodeled high frequency dynamics. We assume that some informations is available concerning high frequency dynamics which are modeled as the fast subsystem.

The $H^{\infty}$ design method introduces additional states to construct the generalized plant, so that the control input does not excite the high frequency unmodeled dynamics. The advantage of using this method with singularly perturbed systems is that we can construct a sliding mode controller for the slow subsystem and take the fast subsystem as a high frequency disturbance. Doing so allows us to choose the appropriate weighting function, which is key in designing a good $H^{\infty}$ controller. Choosing an appropriate weighting function is one of the design difficulties associated with $H^{\infty}$ control [46]. However, by using the $H^{\infty}$ frequency shaped sliding mode controller for singularly perturbed systems one can then overcome the difficulties in choosing the appropriate weighting matrix.

In this chapter, the first approach by Utkin [57] is briefly reviewed in section 6.2 . Section 6.3 discusses the second approach where a frequency shaped sliding surface is reviewed. Sections 6.4 and 6.5 focus on the solution that we introduce to solve the problem of surface parameter design for a single sliding mode controller for the full order 
singularly perturbed model. Using the second approach we will design a sliding mode controller for the slow subsystem by using an $H^{\infty}$ frequency shaping method and we will discuss the results.

\subsection{Sliding mode using LQ approach}

This first approach will use $H^{\infty}$ control to design the sliding surface in the sliding mode controller. It is useful to review this approach to understand how optimal control techniques can be used to determine the surface parameters in a sliding mode controller.

A linear control law based on a quadratic cost function is well known as linear quadratic optimal control. Utkin and Young [57] have applied this method to design the surface for a sliding mode controller. We consider the following linear time invariant system,

$\dot{\boldsymbol{x}}=A \boldsymbol{x}+B \boldsymbol{u}$

where $A \in R^{n \times n}$ and $B \in R^{n \times m}$. The cost functional to be minimized is given by,

$J=\int_{t_{s}}^{\infty} x^{T} Q x d t$

where $t_{s}$ is the time at which sliding mode begins and $Q$ is a symmetric positive definite matrix. Using the state variable transformation $T$ which has the form,

$T^{-1} B=\left[\begin{array}{c}0 \\ B_{2}\end{array}\right]$

Equation (6.1) can be rewritten as,

$$
\left[\begin{array}{l}
\dot{x}_{1} \\
\dot{x}_{2}
\end{array}\right]=\left[\begin{array}{ll}
A_{11} & A_{12} \\
A_{21} & A_{22}
\end{array}\right]\left[\begin{array}{l}
x_{1} \\
x_{2}
\end{array}\right]+\left[\begin{array}{c}
0 \\
B_{2}
\end{array}\right] u
$$


which is known as regular form [10]. The cost function given by equation (6.2) can be rewritten as

$$
J=\int_{t_{s}}^{\infty}\left(x_{I}^{T} Q_{11} x_{I}+2 x_{I}^{T} Q_{I 2} x_{2}+x_{2}^{T} Q_{22} x_{2}\right) d t
$$

where $x_{1} \in R^{n-m}, x_{2} \in R^{m}$ and $Q=\left[\begin{array}{ll}Q_{11} & Q_{12} \\ Q_{12} & Q_{22}\end{array}\right]$

The sliding surface $S=0$ of the sliding mode can be determined so as to minimize the cost functional given by equation (6.5). This problem can be regarded as a linear state feedback control design for the following subsystem

$\dot{x}_{1}=A_{11} x_{1}+A_{12} x_{2}$

with the cost function (6.5). In equation (6.6) $x_{2}$ is considered to be the input of the subsystem and the state feedback controller is $x_{2}=K x_{1}$. This subsystem gives the sliding surface for the total subsystem, namely $S=x_{2}-K x_{1}$. For simplicity we assume $Q_{12}=Q_{21}^{T}=0$. The optimal sliding surface under these conditions is given by [57],

$$
\begin{aligned}
& S=x_{2}+Q_{22}^{-1} A_{12}^{T} P x_{1}=0 \\
& K=-Q_{22}^{-1} A_{12}^{T} P
\end{aligned}
$$

where $P>0$ is a unique solution of following Riccati equation,

$$
P A+A^{T} P-P A_{12} Q_{22}^{-1} A_{12}^{T} P+Q_{11}=0
$$

For the existence of the solution of the Riccati equation (6.9), the pair $\left(A_{11}, A_{12}\right)$ must be controllable and the pair $\left(Q_{I I}^{(1 / 2)}, A_{H}\right)$ must be observable, $Q_{I I} \geq 0$ has to be semi positive definite and $Q_{22}>0$ (Utkin [57]) 


\subsection{Frequency shaped LQ approach}

In this section we review the second method, which is frequency shaping of the sliding mode dynamics through the introduction of compensator dynamics in the sliding surface design. This type of sliding surface is used to penalize the high frequency control actions and thus the unmodeled dynamics are minimally excited. This approach was first introduced by Gupta [21] to handle the flexibility in a spacecraft. Then it was modified by Young [61] and was demonstrated in a practical example (robot manipulator with flexible joint). The cost function given by equation (6.5) can be rewritten in the frequency domain using Parseval's theorem [32][61] as,

$J=\frac{1}{2 \pi} \int_{-\infty}^{\infty}\left(x_{1}^{*}(j \omega) Q_{11} x_{1}(j \omega)+x_{2}^{*}(j \omega) Q_{22} x_{2}(j \omega)\right) d \omega$

where we assume $Q_{12}=Q_{21}^{T}=0$. In the frequency domain a frequency dependent weighting matrix $Q_{22}(j \omega)$ is introduced so that the control inputs for certain frequencies can be amplified or suppressed. We can choose $Q_{22}$ to yield the reduction of high frequency control inputs to the subsystem given by equation (6.6). This approach is realized using a state space representation. The transfer function matrix $W_{2}(s)$ is defined as

$Q_{22}(j \omega)=W_{2}^{*}(j \omega) W_{2}(j \omega)$

where $W_{2}^{*}(s)$ stands for the conjugate transpose of $W_{2}(s)$. The frequency shaped input $\tilde{u}$ is then given by,

$\tilde{u}=W_{2}(s) x_{2}$ 
$W_{2}(s)$ can be represented by the following standard state space representation

$\dot{x}_{W_{2}}=A_{W_{2}} x_{W_{2}}+B_{W_{2}} x_{2}$

$\tilde{u}=C_{W_{2}} x_{W_{2}}+D_{W_{2}} x_{2}$

Then the cost function given by equation (6.10) can be rewritten as [32],

$J=\frac{1}{2 \pi} \int_{-\infty}^{\infty}\left(x_{1}^{*}(j \omega) Q_{11} x_{1}(j \omega)+\left(W_{2}(j \omega) x_{2}(j \omega)\right)^{*} W_{2}(j \omega) x_{2}(j \omega)\right) d \omega$

Rewrite equation (6.15) in the time domain as,

$J=\int_{t_{s}}^{\infty}\left(x_{1}^{T}(t) Q_{11} x_{1}(t)+\tilde{u}^{T}(t) \tilde{u}(t)\right) d t$

then the following extended plane can be used [32],

$\dot{x_{e}}=A_{e} x_{e}+B_{e} x_{2}$

where $\boldsymbol{x}_{e}=\left[\begin{array}{c}\boldsymbol{x}_{W_{2}} \\ \boldsymbol{x}_{1}\end{array}\right], A_{e}=\operatorname{diag}\left(A_{W_{2}}, A_{11}\right)$ and $B_{e}=\left[\begin{array}{c}B_{W_{2}} \\ A_{12}\end{array}\right]$.

Consider $Q_{e}=\left[\begin{array}{cc}C_{W_{2}}^{T} C_{W_{2}} & 0 \\ 0 & Q_{11}\end{array}\right], N_{e}=\left[\begin{array}{c}C_{W_{2}}^{T} D_{W_{2}} \\ 0\end{array}\right]$ and $R_{e}=D_{W_{2}}^{T} D_{W_{2}}$.

Then the cost function given by equation (6.15) is converted to,

$$
J=\int_{t_{s}}^{\infty}\left(x_{e}^{T}(t) Q_{e} x_{e}(t)+2 x_{e}^{T}(t) N_{e} x_{2}(t)+x_{2}^{T}(t) R_{e} x_{2}(t)\right) d t
$$

Minimization of the cost function with cross terms between state and control input is achieved by solving the Riccati equation

$$
P_{e} A_{e}+A_{e} P_{e}-\left(P_{e} B_{e}+N_{e}\right) R_{e}^{-1}\left(B_{e}^{T} P_{e}+N_{e}^{T}\right)+Q_{e}=0
$$

The optimal sliding surface using the solution of equation (6.19) is,

$$
S=x_{2}+R_{e}^{-1}\left(B_{e}^{T} P_{e}+N_{e}^{T}\right) x_{e}
$$


Therefore, the controller gains are given by

$$
K=R_{e}^{-1}\left(B_{e}^{T} P_{e}+N_{e}^{T}\right)
$$

For $K=\left[\begin{array}{ll}K_{1} & K_{2}\end{array}\right]$ equation (6.20) can be rewritten as

$$
S=x_{2}+K_{1} x_{w_{2}}+K_{2} x_{1}=0
$$

which is a linear operator of the states and is the sliding surface of the extended system,

$$
\dot{\tilde{x}}=\tilde{A} \tilde{\boldsymbol{x}}+\tilde{B} u
$$

with $\tilde{\boldsymbol{x}}=\left[\begin{array}{c}\boldsymbol{x}_{W_{2}} \\ \boldsymbol{x}_{1} \\ \boldsymbol{x}_{2}\end{array}\right], \tilde{A}=\left[\begin{array}{ccc}A_{W_{2}} & 0 & B_{W_{2}} \\ 0 & A_{11} & A_{12} \\ 0 & A_{21} & A_{22}\end{array}\right]$ and $\tilde{B}=\left[\begin{array}{c}0 \\ 0 \\ B_{2}\end{array}\right]$.

Therefore, we have designed a controller based on a frequency weighted cost function given by equation (6.15) and the optimal controller is given by equation (6.21).

\subsection{Review of the $H^{\infty}$ approach}

Linear control theory has developed rapidly especially in the field of robust control. $H^{\infty}$ optimal control theory is one of the methods for robust control. The control goal for $H^{\infty}$ optimal control is formulated through a norm minimization of the generalized plant [13], where the $H^{\infty}$ norm is used to formulate the cost function. Figure 6.1 shows the block diagram for the control system in the standard case. 


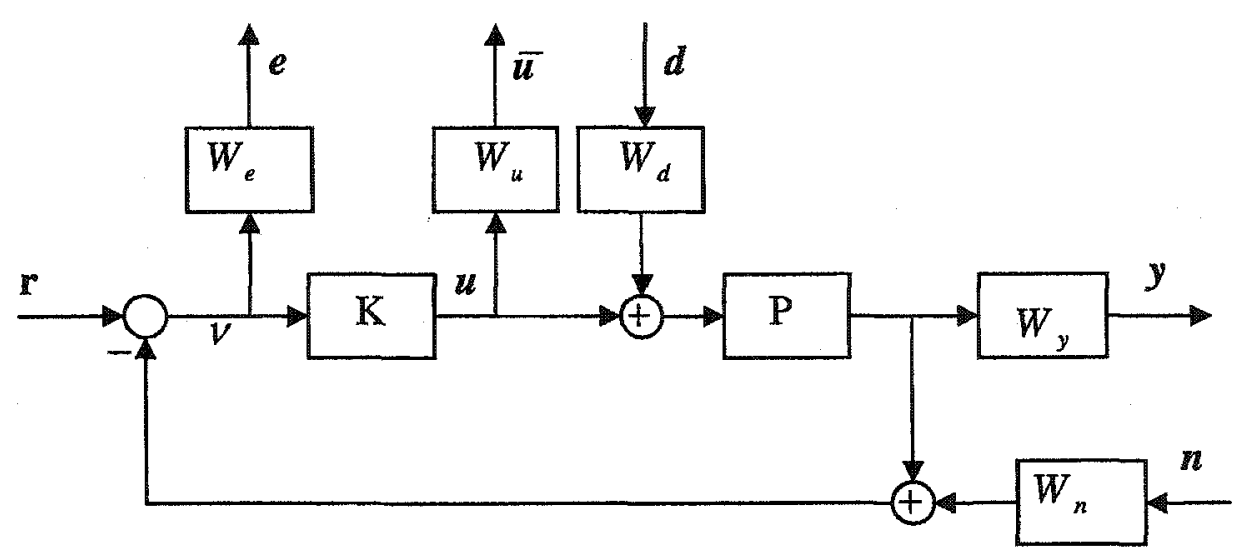

Figure 6.1: Standard weighted closed loop system [63]

There are many advantages of using weighted performance specifications in multivariable system design. For example some components of a vector signal are usually more important than others. Also we could be interested in rejecting errors in a certain frequency range (for example, high frequencies) thus we use frequency dependent weighting. The transfer function of the open loop system in Figure 6.1 can be obtained from the following equation,

$$
\begin{aligned}
& \boldsymbol{v}=\boldsymbol{r}-\left(P\left[W_{d} d+\boldsymbol{u}\right]+W_{n} \boldsymbol{n}\right) \\
& \boldsymbol{y}=W_{y}\left(P\left[W_{d} d+u\right]\right) \\
& \boldsymbol{e}=W_{e} y \\
& \bar{u}=W_{u} u
\end{aligned}
$$

Denoting the transfer function matrix as $G$ we get,

$$
G=\left[\begin{array}{cccc}
W_{e} & -W_{e} P W_{d} & -W_{e} W_{n} & -W_{e} P \\
0 & 0 & 0 & W_{u} \\
1 & -P W_{d} & -W_{n} & -P \\
0 & W_{y} P W_{d} & 0 & W_{y} P
\end{array}\right]
$$


where $\left[\begin{array}{l}e \\ \bar{u} \\ v \\ y\end{array}\right]=G\left[\begin{array}{l}r \\ d \\ n \\ u\end{array}\right]$

The majority of the effort in designing an $H^{\infty}$ robust controller lies in the choice of the weighting functions. A detailed discussion on the technique for choosing these functions can be found in [63]. The output signal $e$ and $\bar{u}$ denote the weighted tracking error and weighted output of the controller $K$.

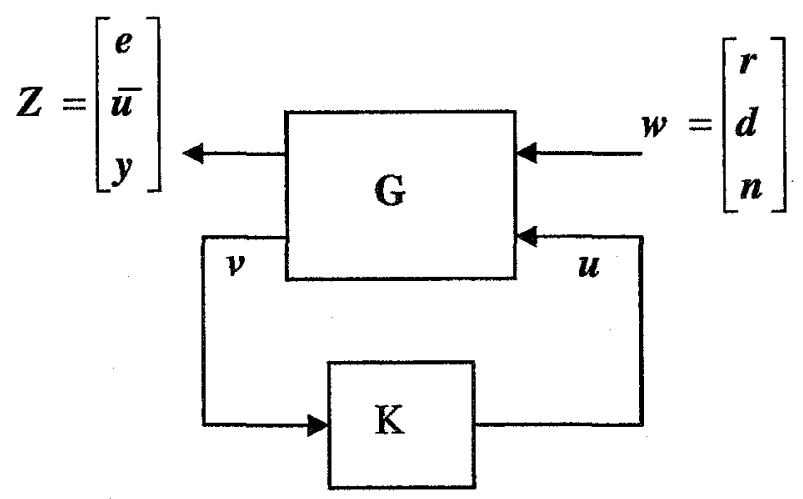

Figure 6.2: Linear Fraction Transformation (LFT)

The above model can be changed to a linear fractional transformation representation [46] as shown in Figure 6.2. Linear fraction transformation is a convenient tool to formulate many mathematical objects. Here, the goal is to create an internally stabilizing controller $K$ such that the worst case output $Z$ due to a class of exogenous disturbances $w$ is kept below a given threshold [46]. This can be done by obtaining the norm between $w$ and $Z$ as a measurement of performance.

The inputs to the plant have been partitioned into two groups: $w$, containing disturbance and reference signals, and $u$, the control inputs. Similarly, the vector $Z$ represents the outputs subject to performance specification and $y$ contains the outputs 
available to the controller. Based on the linear fractional transformation approach equations (6.24)- (6.29) can be rewritten in the corresponding state space realization of $T(s)$ as,

$T(s)=\left[\begin{array}{l|ll}A & B_{1} & B_{2} \\ \hline C_{1} & D_{11} & D_{12} \\ C_{2} & D_{21} & D_{22}\end{array}\right]=\left[\begin{array}{ll}G_{11} & G_{12} \\ G_{21} & G_{22}\end{array}\right]$

where the pairs $\left(A, B_{2}\right)$ and $\left(C_{2}, A\right)$ are stabilizable and detectable [63]. Choosing the state space representation for $W_{u}$ and $W_{e}$ to satisfy $D_{11}=D_{22}=0$ and obtain the new $T(s)$ as,

$T(s)=\left[\begin{array}{ccc}A & B_{1} & B_{2} \\ C_{1} & 0 & D_{12} \\ C_{2} & D_{21} & 0\end{array}\right]$

We make the following assumption on our system [63]

1- $\left(A, B_{1}\right)$ is controllable and $\left(C_{1}, A\right)$ is observable

2- $\left(A, B_{2}\right)$ is stabilizable and $\left(C_{2}, A\right)$ is detectable

3- $D_{12}=\left[\begin{array}{l}0 \\ I\end{array}\right]$ and $D_{21}=\left[\begin{array}{ll}0 & I\end{array}\right]$

4- $\left[\begin{array}{cc}A-j \omega I & B_{2} \\ C_{1} & D_{12}\end{array}\right]$ has full column rank for all $\omega$.

5- $\left[\begin{array}{cc}A-j \omega I & B_{I} \\ C_{2} & D_{21}\end{array}\right]$ has full row rank for all $\omega$.

Assumption (2) is necessary to guarantee the existence of a stabilizing controller. Assumptions (4) and (5) together with (2) guarantee the existence of a solution to the corresponding Riccati equations. Assumption (3) ensures the $H^{\infty}$ problem is nonsingular, 
which is a necessary condition for existence of the controller as explained by Doyle [13]. If $D_{12}$ has full column rank and $D_{21}$ has full row rank but they do not satisfy assumption 3 , a normalizing procedure can be preformed as described in [46].

\subsection{1 $\quad H^{\infty}$ Optimal control design}

When the open loop system has been represented and a weighting function has been chosen to minimize a given norm of the closed loop system, a controller can be designed.

The $H^{\infty}$ norm of a closed loop system $T$ is given by [63],

$\|T\|_{\infty}=\operatorname{Sup}_{\omega \in R} \bar{\sigma}[T(j \omega)]$

where $\bar{\sigma}[T(j \omega)]$ denotes the maximum singular value of the closed loop map $T$. The closed loop system in this case is the linear fraction transformation form for the system given in Figure 6.2 and $T$ is defined by equation (6.30). The design of a sub optimal $H^{\infty}$ controller that gives $\|T\|_{\infty}<\gamma$ yields two Hamiltonian matrices

$$
H_{\infty}=\left[\begin{array}{cc}
A & \gamma^{-2} B_{1} B_{1}^{*}-B_{2} B_{2}^{*} \\
-C_{1}^{*} C_{1} & -A^{*}
\end{array}\right]
$$

and

$$
J_{\infty}=\left[\begin{array}{cc}
A^{*} & \gamma^{-2} C_{1}^{*} C_{1}-C_{2}^{*} C_{2} \\
-B_{1} B_{1}^{*} & -A
\end{array}\right]
$$

These Hamiltonian Matrices give rise to two Riccati equations.

$$
\begin{aligned}
& A^{*} x_{\infty}+x_{\infty} A+x_{\infty}\left(\gamma^{-2} B_{1} B_{1}^{*}-B_{2} B_{2}^{*}\right) x_{\infty}+C_{1}^{*} C_{1}=0 \text { and } \\
& A y_{\infty}+y_{\infty} A^{*}+y_{\infty}\left(\gamma^{-2} C_{1}^{*} C_{1}-C_{2}^{*} C_{2}\right) y_{\infty}+B_{1} B_{1}^{*}=0
\end{aligned}
$$


It is useful to notice that the $(1,2)$ blocks of $H_{\infty}$ and $J_{\infty}$ are not sign definite, therefore, a solution to the Riccati equations cannot be guaranteed for all $\gamma$ [13]. The following theorem [6][13][63] guarantees the existence of an $H^{\infty}$ sub optimal controller.

\section{Theorem (6.1) [63]}

There exists an admissible controller such that $\|T\|_{\infty}<\gamma$ if and only if:

I- $H_{\infty} \in \operatorname{dom}($ Ric $)$ and $x_{\infty}=\operatorname{Ric}\left(H_{\infty}\right)>0$

2- $J_{\infty} \in \operatorname{dom}(\operatorname{Ric})$ and $y_{\infty}=\operatorname{Ric}\left(J_{\infty}\right)>0$

3- $\rho\left(x_{\infty} y_{\infty}\right)<\gamma^{2}$

The $H^{\infty}$ sub-optimal controller is given by,

$$
K=\left[\begin{array}{c|c}
A_{\infty} & -z_{\infty} I_{\infty} \\
\hline F_{\infty} & 0
\end{array}\right]
$$

where $A_{\infty}=A+\gamma^{-2} B_{1} B_{1}^{*} x_{\infty}+B_{2} F_{\infty}+z_{\infty} L_{\infty} C_{2}, F_{\infty}=-B_{2}^{*} x_{\infty}$ and $L_{\infty}=-y_{\infty} C_{2}^{*}$

$H_{\infty} \in d o m(R i c)$ indicates that the Hamiltonian matrices have no eigenvalues on the $j \omega$ axis and $A$ is invertible. $x_{\infty}=\operatorname{Ric}\left(H_{\infty}\right)$ means that $x_{\infty}$ is the stabilizing solution of an algebraic Riccati equation associated with matrix $H_{\infty} \cdot \rho\left(x_{\infty} y_{\infty}\right)$ is the spectral radius of $\left(x_{\infty} y_{\infty}\right)$ thus $\rho\left(x_{\infty} y_{\infty}\right)=\max _{I \leq i \leq n}\left|\lambda_{i}\right|$ where the $\lambda_{i}$ are the eigenvalues of $\left(x_{\infty} y_{\infty}\right)$ which means that $x_{\infty}$ and $y_{\infty}$ are decreasing functions of $\gamma$. Furthermore details can be found in [63] chapter 12. The controller given in equation (6.37) is sub-optimal in that it gives a closed loop system with an $H_{\infty}$ norm less than $\gamma$, the Matlab routine "hinfsyn " can be utilized to satisfies $\|T\|_{\infty}<\gamma$ and the assumptions of theorem (6.1). This gives a control design method which is close to optimal. 


\subsection{Frequency shaped sliding mode control for singularly}

\section{perturbed systems}

The main goal is to use frequency shaped sliding mode control to overcome the sliding surface design problem as discussed in chapter 5 section 5.1. Using a singularly perturbed system actually gives us an advantage and solves one of the complicated design problems for frequency shaped sliding mode control. As has been mentioned earlier in this chapter, the key of good design for frequency shaped sliding mode controllers lies in the choice of weighting functions (Section 6.4). The selection of the weighting functions for this specified design problem often involves many iterations and fine-tuning. It is very hard to give a general formula or method for the weighting function that will work in this case. The frequency shaped sliding mode control that was discussed by many authors is based on transferring the overall system into regular form then using the upper subsystem to design the sliding surface. Choosing the weighting function in this case is completely up to the designer and his/her experience with the system.

The singular perturbation method provides some information regarding the high frequency dynamics of the system. By analyzing the frequency response of the fast subsystem, we could obtain the required filter that must be working as the weighting functions. For our purposes we will propose the block diagram shown in Figure 6.3. In this case we are introducing the high frequency dynamics as a disturbance. The most important issue in our case is to suppress the high frequency components in the control law $u$. This causes less of an effect on the unmodeled high frequency dynamics. The weighting matrix $W_{u}$ may be used to set some restrictions on the control or actuator 
signal. $W_{e}$ is used to set the requirements on the shape of certain closed loop transfer functions since the weighting function $W_{e}$ affects the input-output relationship by changing the error signal shape.

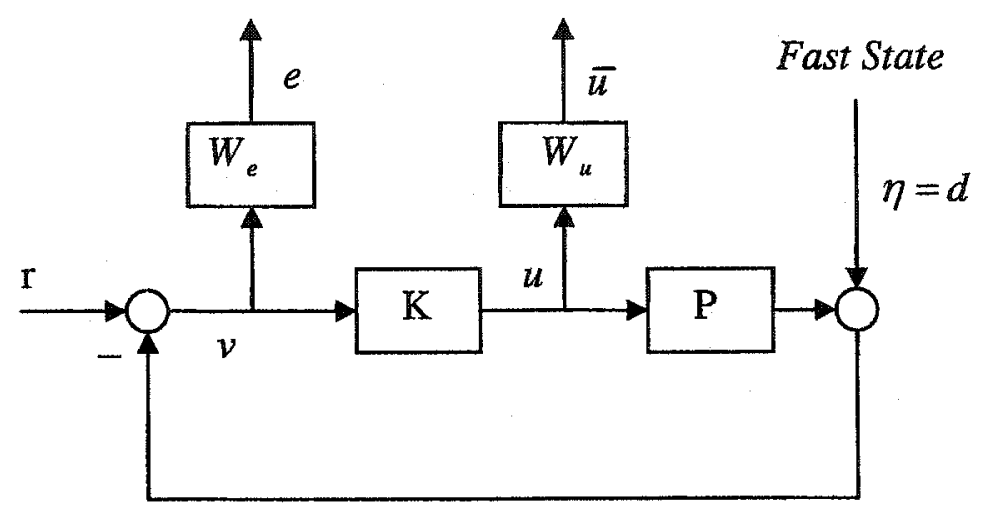

Figure 6.3: Custom weighted closed loop system

Consider a system given by,

$\dot{\boldsymbol{x}}=A_{1 I} \boldsymbol{x}+A_{12} z+B_{1} \boldsymbol{u}$

$\varepsilon z^{*}=A_{21} x+A_{22} z+B_{2} u$

where $x \in R^{n}, z \in R^{m}, u \in R^{r}$ and $\varepsilon$ is a small positive parameter. Applying the quasi steady state decoupling method as has been described in Chapter 3, then we have,

$\dot{\tilde{x}}=A_{11} \tilde{x}+A_{I 2} \tilde{z}+B_{J} \tilde{u}$

$0=A_{21} \tilde{x}+A_{22} \tilde{z}+B_{2} \tilde{u}$

where the symbol " " indicates that $\varepsilon=0$. Thus we can get the following reduced system [9],

$$
\dot{x}_{s}=A_{0} x_{s}+B_{0} u_{s},
$$

where $\quad x_{s}=\tilde{x}$,

$$
A_{0}=A_{11}-A_{12} A_{22}^{-1} A_{21}
$$$$
B_{0}=B_{1}-A_{12} A_{22}^{-1} B_{2}
$$

and

$z_{s}=h\left(x_{s}\right)=-A_{22}^{-1}\left(A_{2 I} x_{s}+B_{2} u_{s}\right)$ which can be seen as the slow part of $z$ given by 
equation (6.39). Assuming that the slow variables are constant during fast transients i.e. $\dot{\tilde{z}}=0$ and $\tilde{x}=x_{s}=$ constant, the approximated fast subsystem for the system given by equations (6.38) and (6.39) can be defined as,

$$
\varepsilon \dot{x}_{f}=A_{22} x_{f}+B_{2} u_{f}
$$

where $x_{f}=x-\tilde{x}$ and $u_{f}=u-\tilde{u}$. To design a frequency shaped sliding mode controller for the slow subsystem we must ensure that the slow subsystem is in regular form [10]. Appropriate linear transformations [10][19][62] can be used to transform the slow subsystem into regular form if needed. The slow subsystem is given by,

$$
\begin{aligned}
& \dot{x}_{s 1}=A_{01} x_{s 1}+A_{02} x_{s 2}, \\
& \dot{x}_{s 2}=A_{03} x_{s 1}+A_{04} x_{s 2}+B_{r} u,
\end{aligned}
$$

where for simplicity $\boldsymbol{u}=\boldsymbol{u}_{s}$ and $B_{r}$ is nonsingular. The hyper-plane for the above plant is expressed as follows,

$$
\sigma\left(x_{s}\right)=V\left(x_{s 1}\right)+x_{s 2}
$$

where $\sigma\left(x_{s}\right)$ and $V\left(x_{s 1}\right)$ are linear operators [61]. The conventional switching surface $S\left(x_{s}\right)$ as described in chapter 4 and chapter 5 does not have dynamics on the hyper plane. However $\sigma\left(x_{s}\right)$ has some dynamics on the hyper plane, the state space realization of $V\left(x_{s I}\right)$ is given by,

$$
\begin{aligned}
& \dot{x_{c}}=F_{c} x_{c}+G_{c} x_{s I} \\
& V\left(x_{s I}\right)=H_{c} x_{c}+L_{c} x_{s I}
\end{aligned}
$$

From equation (6.44), (6.45) and (6.46)-(6.48) the augmented system becomes 


$$
\begin{aligned}
& {\left[\begin{array}{c}
\dot{x}_{c} \\
\dot{x}_{s 1} \\
\dot{x}_{s 2}
\end{array}\right]=\left[\begin{array}{ccc}
F_{c} & G_{c} & 0 \\
0 & A_{01} & A_{02} \\
0 & A_{03} & A_{04}
\end{array}\right]\left[\begin{array}{l}
x_{c} \\
x_{s 1} \\
x_{s 2}
\end{array}\right]+\left[\begin{array}{c}
0 \\
0 \\
B_{2}
\end{array}\right] u} \\
& \sigma\left(x_{s}\right)=\left[\begin{array}{ll}
H_{c} & L_{c}
\end{array}\right]\left[\begin{array}{l}
x_{c} \\
x_{s 1}
\end{array}\right]+x_{s 2}
\end{aligned}
$$

The existence of a sliding mode implies,

$$
\sigma=\dot{\sigma}=0
$$

where $\sigma=\sigma\left(x_{s}\right)$. This means that,

$$
\dot{\sigma}=H_{c} \dot{x}_{c}+L_{c} \dot{x}_{s 1}+\dot{x}_{s 2}=0
$$

Using equation (6.49) and equation (6.52), the equivalent control input $u_{e q}$ can be expressed as follows:

$$
\dot{\sigma}=0=H_{c}\left[F_{c} x_{c}+G_{c} x_{s 1}\right]+L_{c} A_{01} x_{s 1}+L_{c} A_{03} x_{s 1}+A_{03} x_{s 1}+A_{04} x_{s 2}+B_{r} u_{e q}
$$

Equation (6.53) can be rewritten as,

$$
u_{e q}=-B_{r}^{-1}\left[H_{c} F_{c} x_{c}+\left(H_{c} G_{c}+L_{c} A_{0 I}+A_{03}\right) x_{s 1}+\left(L_{c} A_{02}+A_{04}\right) x_{s 2}\right]
$$

when the system is on sliding hyper plane, $\sigma=0$, and consequently,

$$
x_{s 2}=-\left[\begin{array}{ll}
H_{c} & L_{c}
\end{array}\right]\left[\begin{array}{l}
x_{c} \\
x_{s I}
\end{array}\right]
$$

From equation (6.49),

$$
\left[\begin{array}{c}
\dot{x}_{c} \\
\dot{x}_{s 1}
\end{array}\right]=\left[\begin{array}{cc}
F_{c} & G_{c} \\
0 & A_{O I}
\end{array}\right]\left[\begin{array}{l}
x_{c} \\
x_{s 1}
\end{array}\right]+\left[\begin{array}{c}
0 \\
A_{02}
\end{array}\right] x_{s 2}
$$

The feedback gain matrix for equation (6.55) and equation (6.56) is $\left[\begin{array}{ll}H_{c} & L_{c}\end{array}\right]$. The reduced order system on the sliding hyper plane can also be expressed as, 
$\left[\begin{array}{c}\dot{x}_{c} \\ \dot{x}_{s I}\end{array}\right]=\left[\begin{array}{cc}F_{c} & G_{c} \\ -A_{02} H_{c} & A_{01}-A_{02} L_{c}\end{array}\right]\left[\begin{array}{c}x_{c} \\ x_{s I}\end{array}\right]$

Similar to equivalent control method described in chapter 2, we choose a Lyapunov function as, $\psi=\frac{1}{2} \sigma^{2}$

For stability it is required that the derivative of $\psi$ be negative, therefore, $\sigma \dot{\sigma}<0$. This is can be done if $\dot{\sigma}=-\eta \sigma \operatorname{sgn}(\sigma)$, where $\operatorname{sgn}(\sigma)$ is the signum function as described in chapter 2 and $\eta$ is an arbitrary positive constant. . Hence using equation (6.52) the stability condition can be written as,

$$
\dot{\sigma}=H_{c} \dot{x}_{c}+L_{c} \dot{x}_{s 1}+\dot{x}_{s 2}=-\eta \sigma \operatorname{sgn}\left(H_{c} x_{c}+L_{c} x_{s 1}+x_{s 2}\right)
$$

Given the values of $\dot{x}_{c}, \dot{x}_{s 1}$ and $\dot{x}_{s 2}$ in equation (6.49) thus equation (6.59) can be rewritten as,

$$
\begin{aligned}
u= & -B_{r}^{-1}\left[H_{c} F_{c} \boldsymbol{x}_{c}+\left(H_{c} G_{c}+L_{c} A_{01}+A_{03}\right) \boldsymbol{x}_{s 1}+\left(L_{c} A_{02}+A_{04}\right) \boldsymbol{x}_{s 2}\right] \\
& -B_{r}^{-l} \eta\left(H_{c} \boldsymbol{x}_{c}+L_{c} \boldsymbol{x}_{s 1}+\boldsymbol{x}_{s 2}\right) \operatorname{sgn}\left(H_{c} \boldsymbol{x}_{c}+L_{c} \boldsymbol{x}_{s 1}+\boldsymbol{x}_{s 2}\right)
\end{aligned}
$$

Figure 6.4 shows block diagram of $H^{\infty}$ frequency shaped sliding mode control.

Young [61] has shown that all eigenvalues of equation (6.57) could be arbitrarily chosen. The compensator $\left\{F_{c}, G_{c}, H_{c}, L_{c}\right\}$ in equation (6.47) and (6.48) is obtained using the $H^{\infty}$ approach as given in Section 6.4. The realization of compensator $\left\{F_{c}, G_{c}, H_{c}, L_{c}\right\}$ can be expressed as follows,

$$
K=\left[\begin{array}{cc}
F_{c} & G_{c} \\
H_{c} & L_{c}
\end{array}\right]
$$


where $\left[\begin{array}{l}\dot{x_{c}} \\ V\end{array}\right]=K\left[\begin{array}{l}x_{c} \\ x_{s 1}\end{array}\right]$

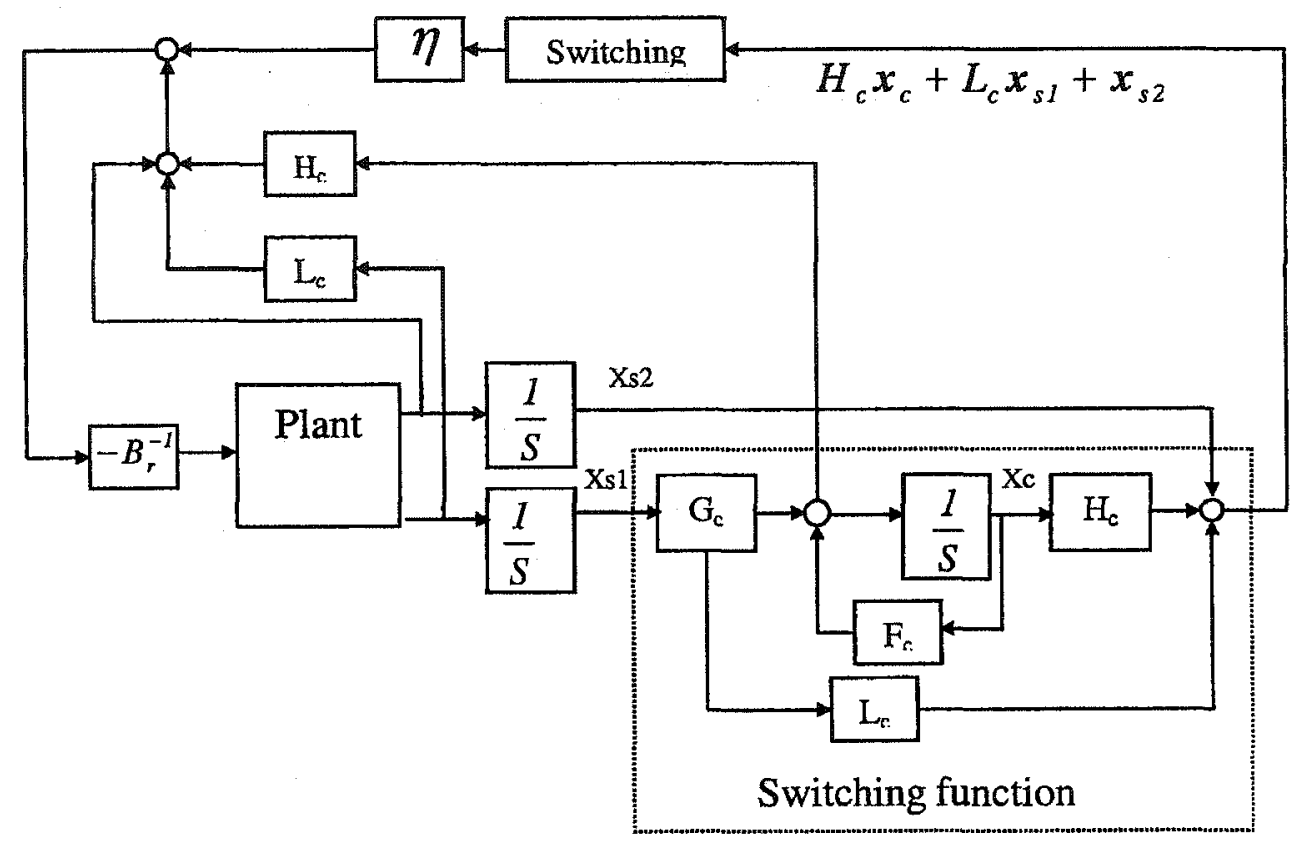

Figure 6.4: $H^{\infty}$ frequency shaped sliding mode control

To demonstrate this method, we use the following example.

\section{EXAMPLE (1)}

Consider a magnetic tape control system given by

$$
\begin{aligned}
& {\left[\begin{array}{l}
\dot{x_{1}} \\
\dot{x_{2}} \\
\varepsilon \dot{z}_{1} \\
\varepsilon \dot{z}_{2}
\end{array}\right]=\left[\begin{array}{cccc}
0 & 0.4 & 0 & 0 \\
0 & 0 & 0.345 & 0 \\
0 & -0.524 & -0.465 & 0.262 \\
0 & 0 & 0 & -1
\end{array}\right]\left[\begin{array}{l}
x_{1} \\
x_{2} \\
z_{1} \\
z_{2}
\end{array}\right]+\left[\begin{array}{l}
0 \\
0 \\
0 \\
1
\end{array}\right] u} \\
& y=\left[\begin{array}{llll}
1 & 0 & 0 & 0 \\
0 & 0 & 1 & 0
\end{array}\right]\left[\begin{array}{l}
x_{1} \\
x_{2} \\
z_{1} \\
z_{2}
\end{array}\right] .
\end{aligned}
$$


This can be put in standard singularly perturbed form as,

$\dot{x}=A_{11} x+A_{12} z+B_{1} u$

$\varepsilon z^{\circ}=A_{12} x+A_{22} z+B_{2} u$,

where $\quad A_{11}=\left[\begin{array}{cc}0 & 0.4 \\ 0 & 0\end{array}\right], \quad A_{12}=\left[\begin{array}{cc}0 & 0 \\ 0.345 & 0\end{array}\right], \quad A_{12}=\left[\begin{array}{cc}0 & -0.524 \\ 0 & 0\end{array}\right]$,

$A_{22}=\left[\begin{array}{cc}-0.465 & 0.262 \\ 0 & -1\end{array}\right]$

$B_{1}=\left[\begin{array}{l}0 \\ 0\end{array}\right], B_{2}=\left[\begin{array}{l}0 \\ 1\end{array}\right]$ and $\varepsilon=0.1$

Setting $\varepsilon=0$ in equation (6.66) gives us the reduced slow subsystem

The slow subsystem is given by,

$\dot{x}_{s}=A_{0} x_{s}+B_{0} u$

where $A_{0}=A_{11}-A_{12} A_{22}^{-1} A_{21}=\left[\begin{array}{cc}0 & 0.4 \\ 0 & -0.38\end{array}\right]$ and $B_{0}=B_{1}-A_{12} A_{22}^{-1} B_{2}=\left[\begin{array}{c}0 \\ 0.1944\end{array}\right]$

which is already in the required regular form. The fast subsystem can be described by

$\dot{x}_{f}=A_{f} x_{f}+B_{f} u_{f}$

where $A_{f}=\left[\begin{array}{cc}-4.222 & 2.62 \\ 0 & -10\end{array}\right]$ and $B_{f}=\left[\begin{array}{c}0 \\ 10\end{array}\right]$

It is required to design a single sliding mode controller based on the slow subsystem to control the full order model. Now let us analyze the fast subsystem. The $\infty$ norm has the form,

$\left\|T_{f}\right\|_{\infty}=\operatorname{Sup}_{\omega \in R} \bar{\sigma}\left[T_{f}(j \omega)\right]$ 
where $T_{f}$ is the transfer function matrix for the fast subsystem given by the system matrix $G_{f}=\left[\begin{array}{l|l}A_{f} & B_{f} \\ \hline C_{f} & D_{f}\end{array}\right]$

where $C_{f}=\left[\begin{array}{ll}1 & 0\end{array}\right]$ and $D_{f}=[0]$. There are many methods to obtain the $\infty$ norm and the singular values of the transfer function $T_{f}(j \omega)$. For details on these methods a complete review can be found in [46]. We found that obtaining the value graphically is most suitable in our case. The value is read directly from a bode singular value plot which can be easily obtained using Matlab.

The $H^{\infty}$ norm of the fast subsystem can be calculated using the Matlab function "hinfnorm" and is given by,

$\left\|T_{f}(s)\right\|_{\infty}=88.7$

where $T_{f}(s)$ is the transfer function matrix for the fast subsystem. This is shown as the peak of the largest singular value bode plot as shown in Figure 6.5. This peak is achieved at frequency $\omega_{\max } \cong 75 \mathrm{~Hz}$. This shows that the system response will be amplified 88.7 times for an input signal at the frequency $\omega_{\max }$. 


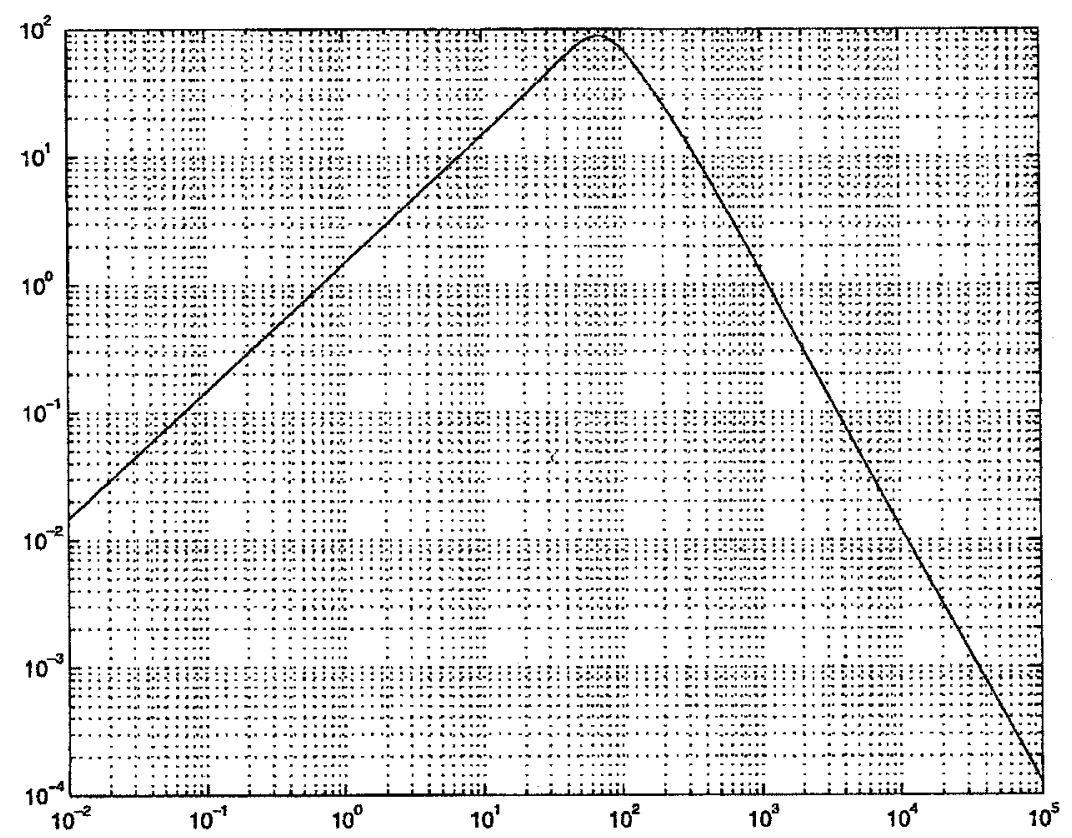

Figure 6.5: The singular value of $\left\|G_{f}(s)\right\|_{\infty}$

Since the fast subsystem is considered as a disturbance, it is desirable to attenuate the excitation of the system at this frequency. $W_{u}$ is chosen to heavily attenuate the frequency components in $u$ that may cause excitation of the high frequency dynamics (fast subsystem).

From Figure 6.5 it is clear that $W_{u}$ should pass frequencies below $1 \mathrm{~Hz}$ and attenuate the frequencies in the $75 \mathrm{~Hz}$ range. We choose $W_{u}$ to be,

$$
W_{u}=\frac{s+98}{10 s+9.8}
$$

The frequency response for $W_{u}$ is shown in Figure 6.6. On the other hand, we choose $W_{e}$ which yields the desired tracking error specification [63]. We must remember the important relationship between $W_{u}$ and $W_{e}$ (i.e. compromise between fast command 
response and low frequency control input). Furthermore, the cutoff frequency of $W_{e}$ and $W_{u}$ must not be close together for the existence of the $H^{\infty}$ sub-optimal controller [63].

As such we choose $W_{e}$ to be,

$$
W_{e}=\frac{10 s+1}{s+10}
$$

Weighting function selection is basically an iterative procedure to satisfy the assumptions given in theorem 6.1. In our case we use fewer iterative steps to obtain the $H^{\infty}$ controller since the frequency of fast dynamics is known. In this thesis we explained three methods for designing the sliding surface for the slow subsystem. The first method is the pole placement technique [10], which is widely used, to satisfy desired closed loop performance. This method sometimes needs to transform the system into another form using a linear transformation to help in the design process (regular form).

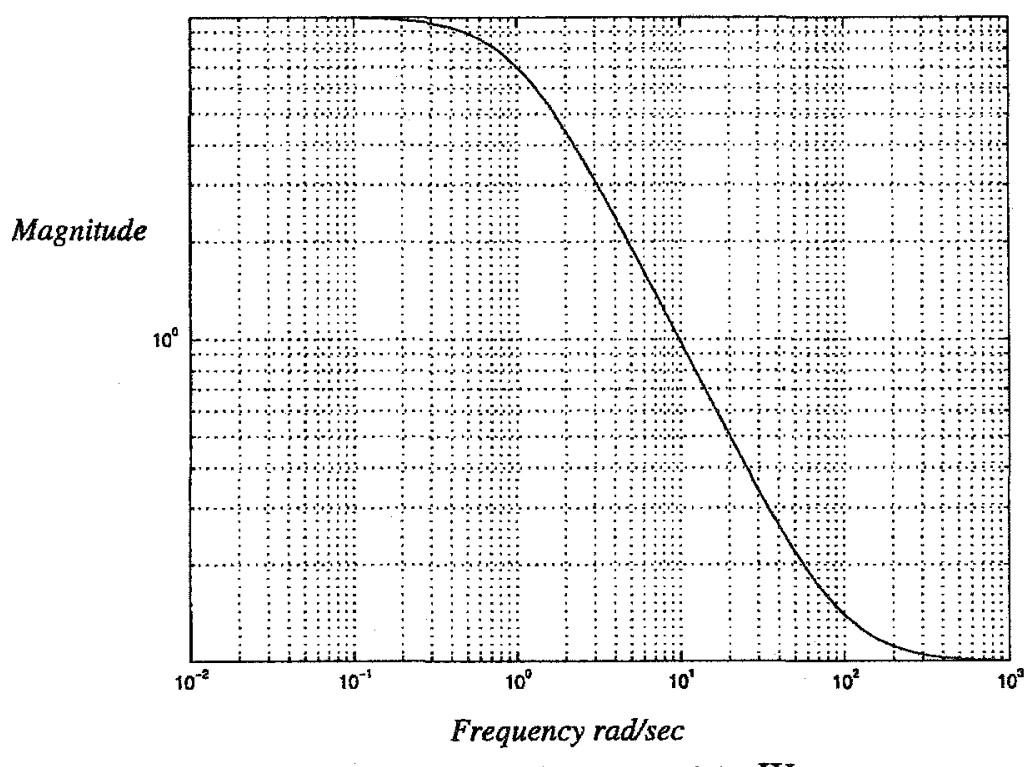

Figure 6.6: Control weight $W_{u}$ 


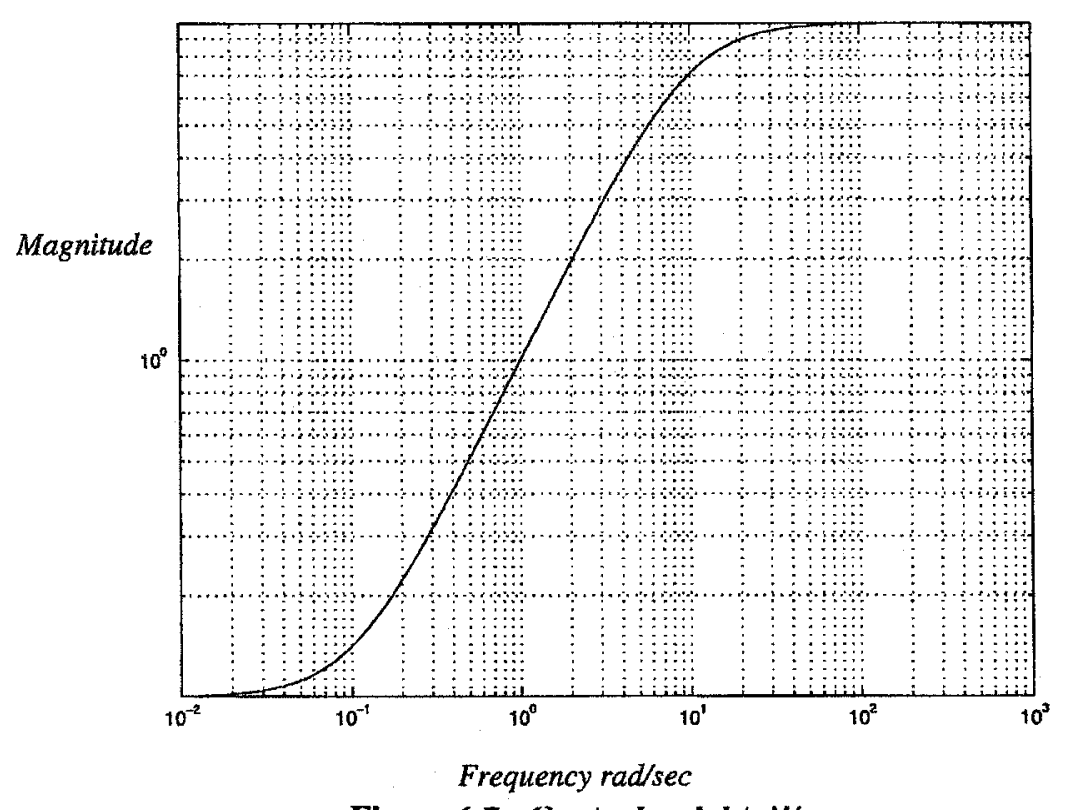

Figure 6.7: Control weight $W_{e}$

The pole placement technique can be done by assigning the desired closed loop poles to the following equation,

$\dot{x}_{s}=\left[I-B_{0}\left(C_{s} B_{0}\right)^{-1} C_{s}\right] A_{0} x_{s}=A_{e q} x_{s}$

Hence we choose $C_{s}$ to ensure that the $(n-r)$ eigenvalues of the equivalent system given by equation (6.73) have negative real parts. The control law will have the form,

$u_{s}=u_{e q}+u_{N}=-\left[\left(C_{s} B_{0}\right)^{-1} C_{s}\right] A_{0} x-\left(C_{s} B_{0}\right)^{-1} \eta_{I} \operatorname{sgn}\left(S_{s}\right)$

where $\boldsymbol{x}$ is replaced with $\boldsymbol{x}_{s}$ in the above equation as described in chapter 3 .

The second method is the tracking control method [48], where we define the surface parameter based on the fast subsystem and this is done by taking $\lambda$ in equation (2.18) to be lower than the slowest eigenvalue of the fast subsystem in the model.

The third method is the frequency shaped sliding surface described as in the above section. Figure 6.8 shows the weighted feedback closed loop model for the slow subsystem. Using the information provided above for $W_{e}, W_{u}$ and the plant then we 
could easily determined the value of $K$ given by equation (6.61). This is done in our case using Matlab functions "augtf" and "Hinf".

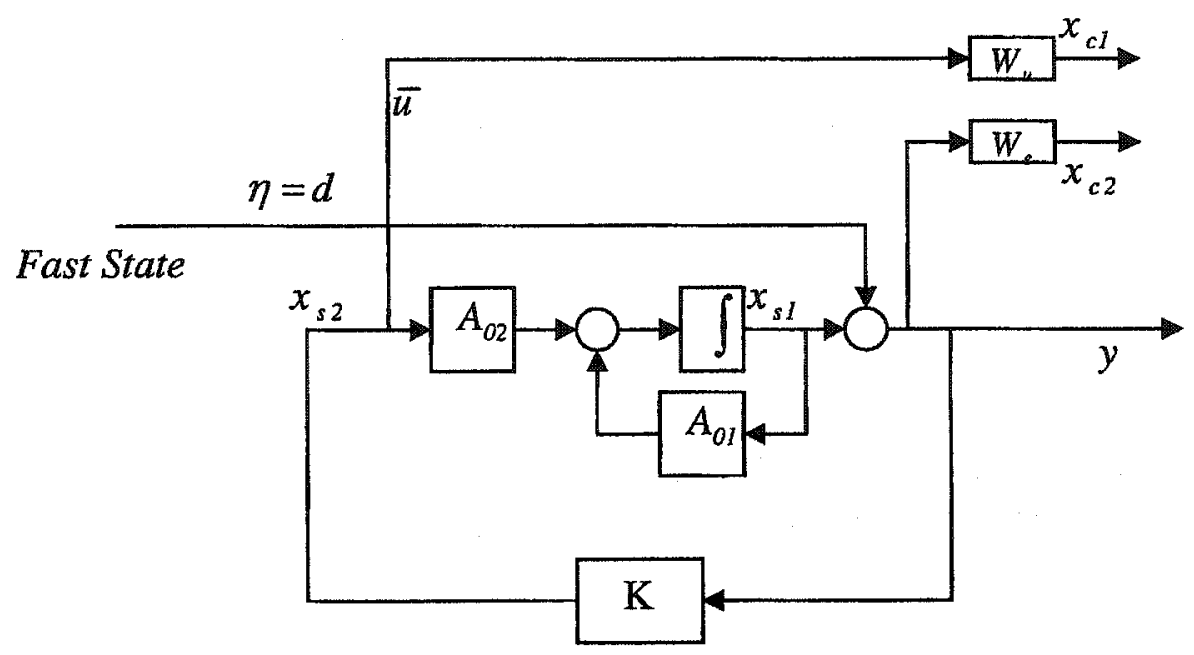

Figure 6.8: Generalized plant for design of $H^{\infty}$ weighted closed loop slow subsystem

The $H^{\infty}$ controller designed for the reduced slow system is given by,

$\dot{x}_{s}=A_{0} x_{s}+B_{0} u$

where $A_{0}=\left[\begin{array}{ll}A_{01} & A_{02} \\ A_{03} & A_{04}\end{array}\right]=\left[\begin{array}{cc}0 & 0.4 \\ 0 & -0.38\end{array}\right]$

The $H^{\infty}$ controller has been obtained using the "hinf" command in Matlab. The $H^{\infty}$ controller has the form,

$K=\left[\begin{array}{ll}F_{c} & G_{c} \\ H_{c} & L_{c}\end{array}\right]$

For our example

$F_{c}=\left[\begin{array}{ccc}-0.708 & 0.8246 & -2.6706 \\ 1.0459 & -1.3665 & 4.1156 \\ 0.2353 & -0.3096 & -0.9986\end{array}\right]$ 
$G_{c}=\left[\begin{array}{l}-0.6781 \\ -1.9401 \\ -0.4184\end{array}\right]$

$H_{c}=\left[\begin{array}{lll}2.0143 & -2.2881 & 7.2007\end{array}\right]$

$L_{c}=0$

Substituting these values into the system given by equation (6.49) and equation (6.50) gives the extended closed loop system. The result from this method is compared with the other methods that we used in the former chapters.

Based on Slotine's recommendation we choose the surface parameters for our controller based on the fast subsystem eigenvalues. Thus the surface parameter for our controller is taken to be $C_{s}=\left[\begin{array}{ll}4 & 1\end{array}\right]$. This selection, however, results in instability as shown in Figure 6.10. Trying to avoid this instability we decreased the slope to a lower value and the surfaces parameter were taken to be $C_{s}=\left[\begin{array}{ll}3 & 1\end{array}\right]$. This choice for surface parameters resulted in a stable system. We point to this approach as "Slotine_2" in Figure 6.9 that compares the difference between the design methods.

Figure 6.9 shows the closed loop time response for the above example where, $\left[\begin{array}{l}x_{s 1}(0) \\ x_{s 2}(0)\end{array}\right]=\left[\begin{array}{l}-0.2 \\ -0.6\end{array}\right],\left[\begin{array}{l}x_{f 1}(0) \\ x_{f 2}(0)\end{array}\right]=\left[\begin{array}{l}0 \\ 0\end{array}\right]$ and the boundary layer $\phi=0.05$

The choice of surface parameters depends on the weighting functions, which are chosen to attenuate the fast modes in the full order model. The choice of sliding surface parameters using the other design methods discussed in this thesis (Slotine's approach, equivalent control method) must be carefully selected in our case otherwise it will lead to unstable results as shown in Figure 6.10. Assigning smaller values of surface parameters 
could solve this problem but this effects the time response of the overall closed loop model.

The main advantage of using the frequency shaped sliding mode controller is to avoid exciting the high frequency dynamics and at the same time obtain the best steady state response compared with other design methods. Exciting the high frequency dynamics can cause the full order closed loop model to be unstable. This has been demonstrated in Figure 6.10.

We found that, it is possible to obtain a time response using the equivalent control method similar to the response obtained using $H^{\infty}$ frequency shaped sliding mode control. This can be done by iteratively tuning the linear sliding surface until we reach approximately the same time response. This is shown in Figures 6.11, 6.12 and 6.13. For the same response in the slow states Figures 6.14 and 6.15 show the difference between the two approaches in the fast state. The fast states are minimally excited in the case of $H^{\infty}$ frequency shaped sliding mode control. This is consistent with the theory. Figure 6.16 shows the phase plane plot in the case of frequency shaped sliding mode controller whereas Figure 6.17 shows the phase plot in case of linear surface with $C_{s}=\left[\begin{array}{ll}1.85 & 1\end{array}\right]$. 


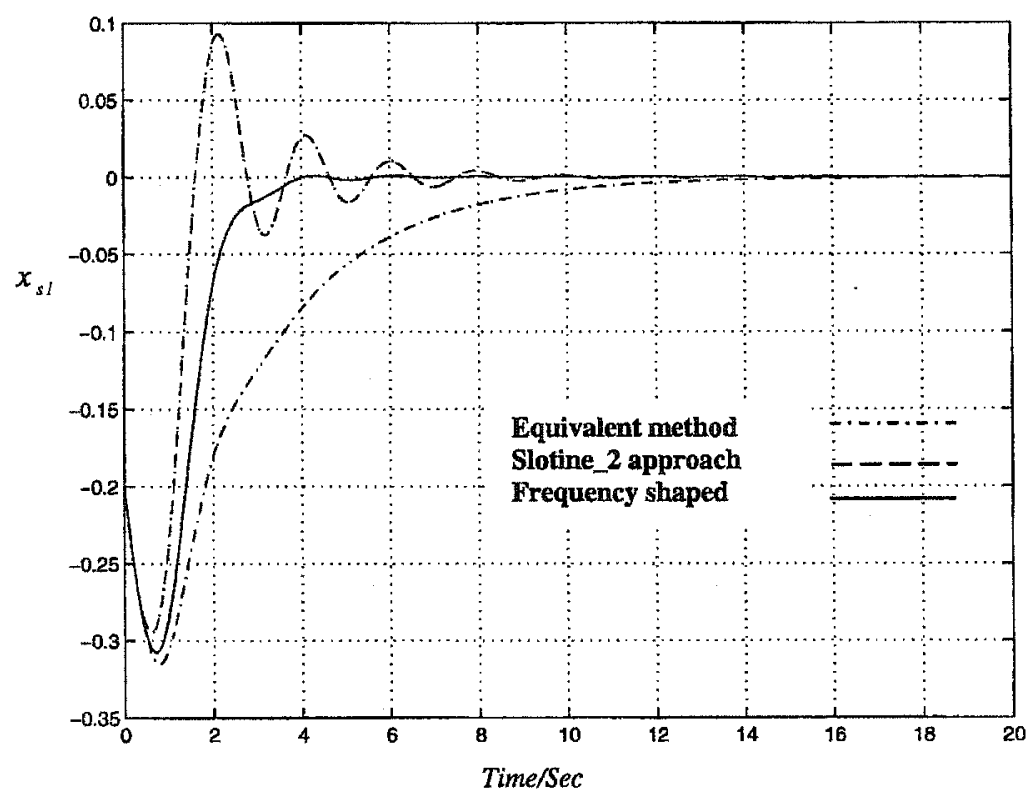

Figure 6.9.a: Closed loop time response of $x_{s 1}$

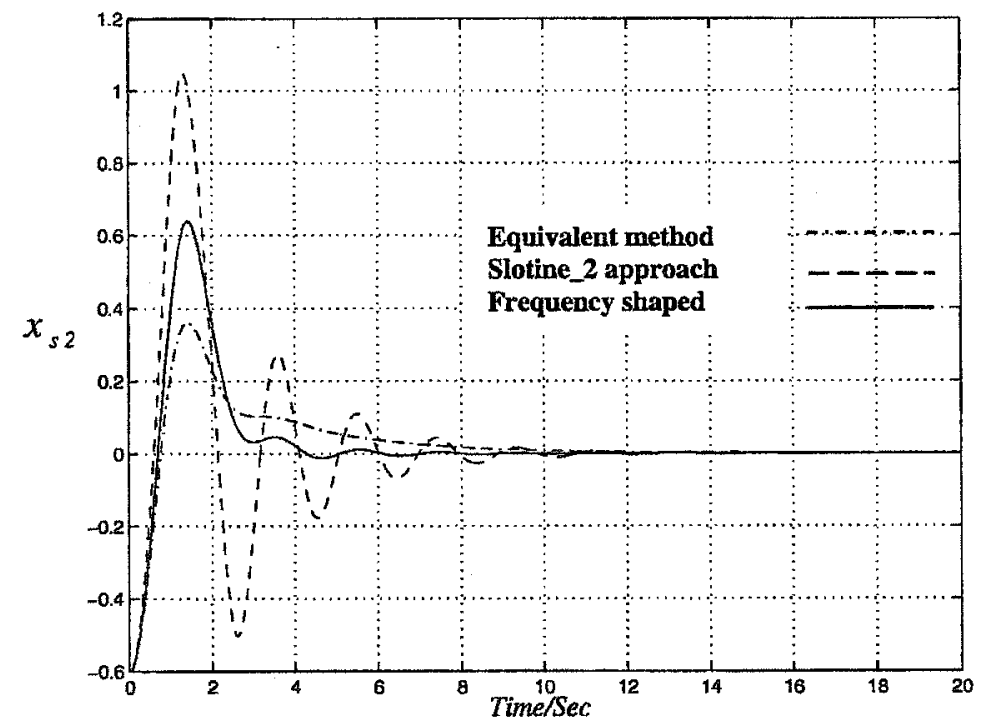

Figure 6.9.b: Closed loop time response of $x_{s 2}$ 


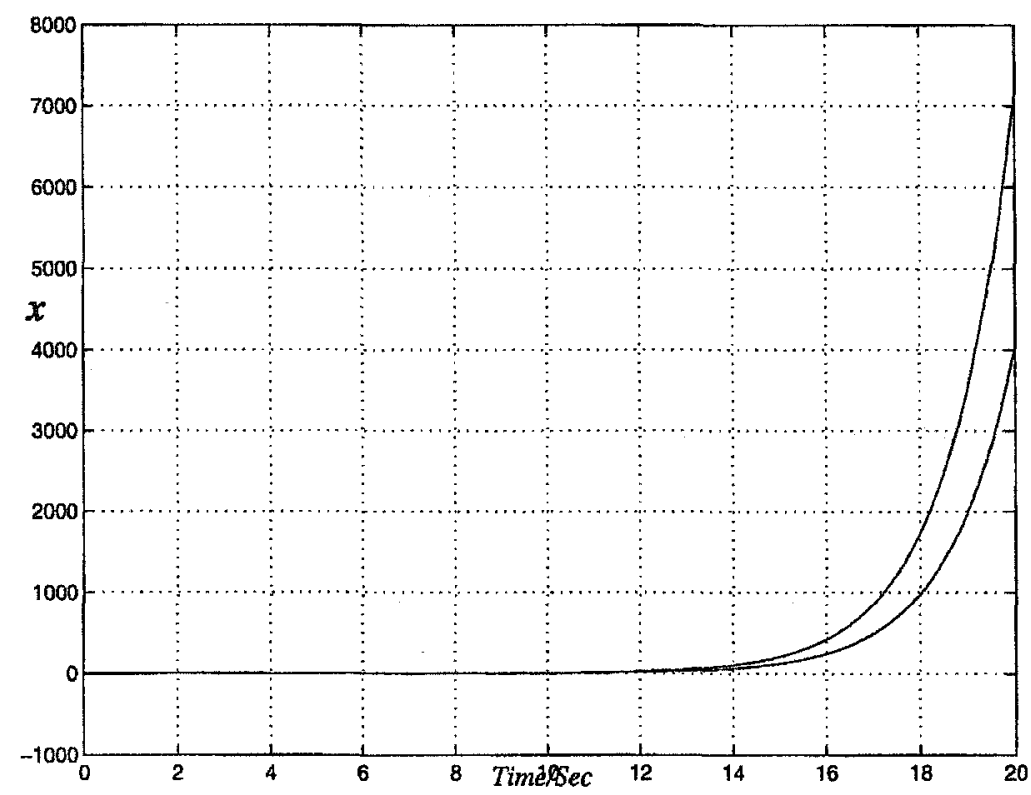

Figure 6.10.a: Closed loop time response of $x$

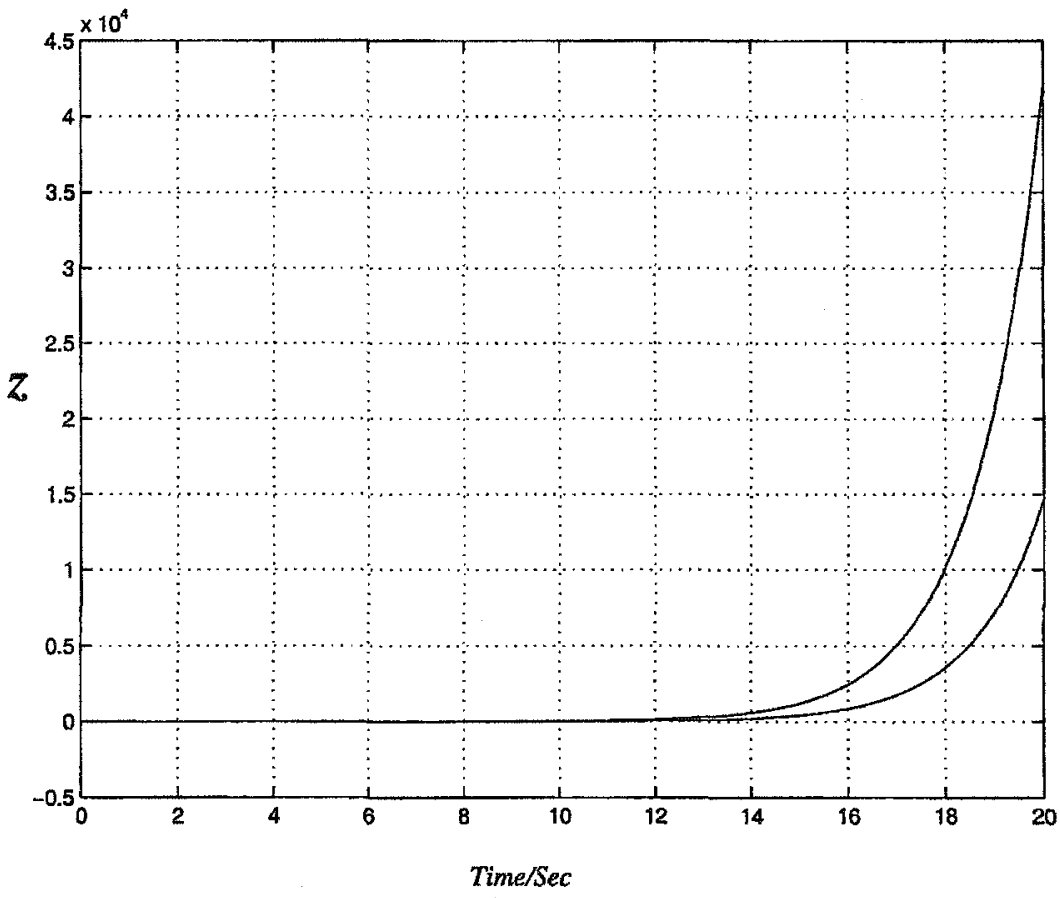

Figure 6.10.b: Closed loop time response of $z$ 


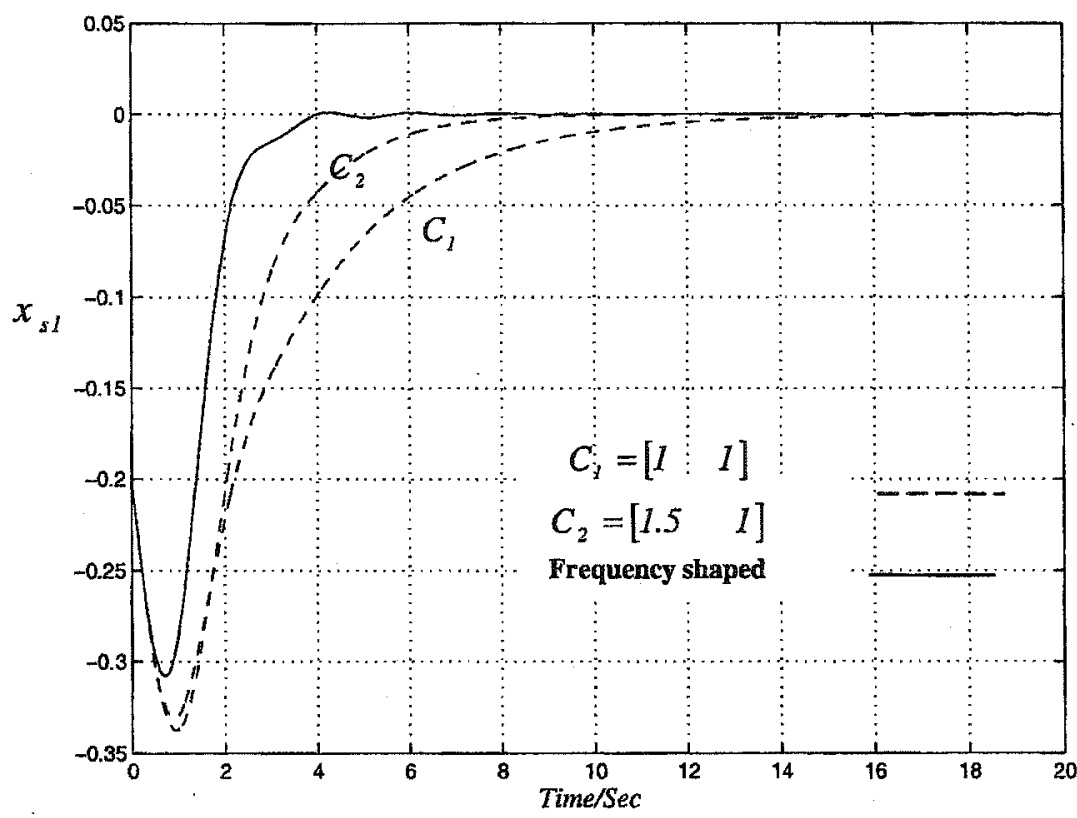

Figure 6.11: Closed loop time response of slow state for different sliding surface

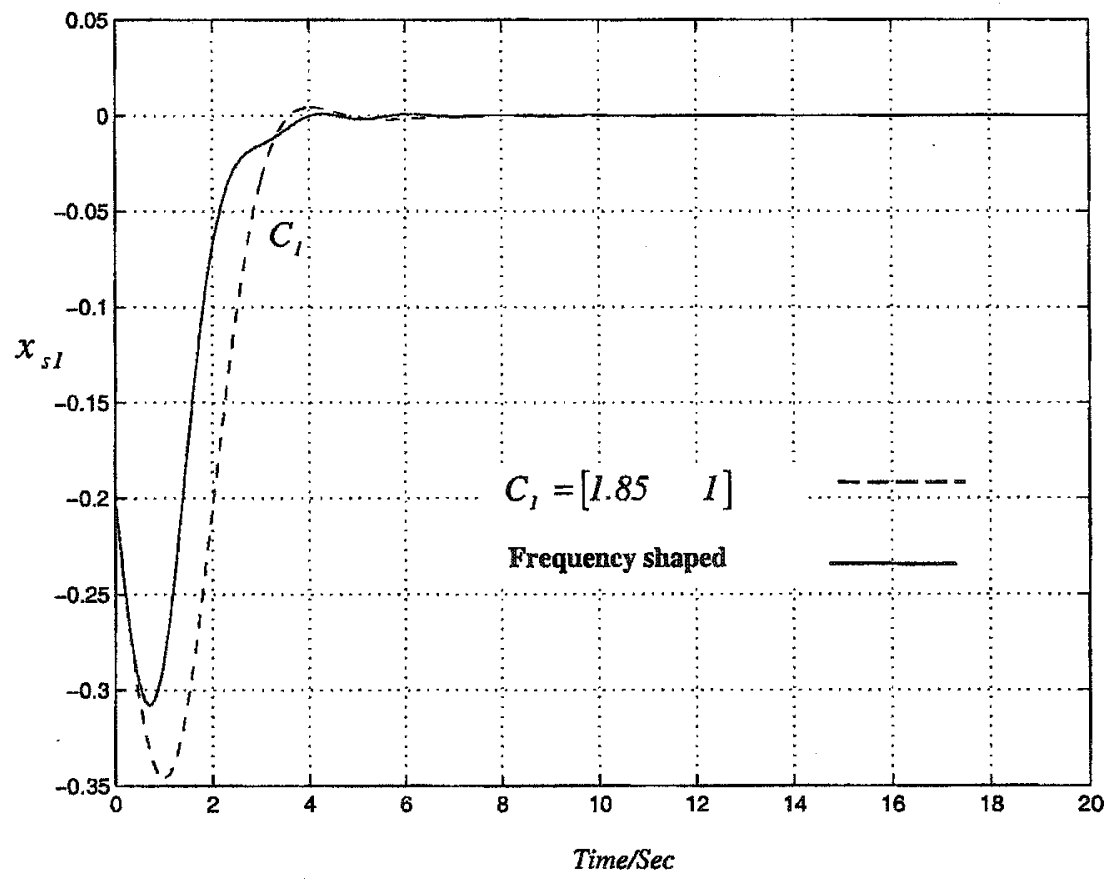

Figure 6.12: Closed loop time response of slow state 


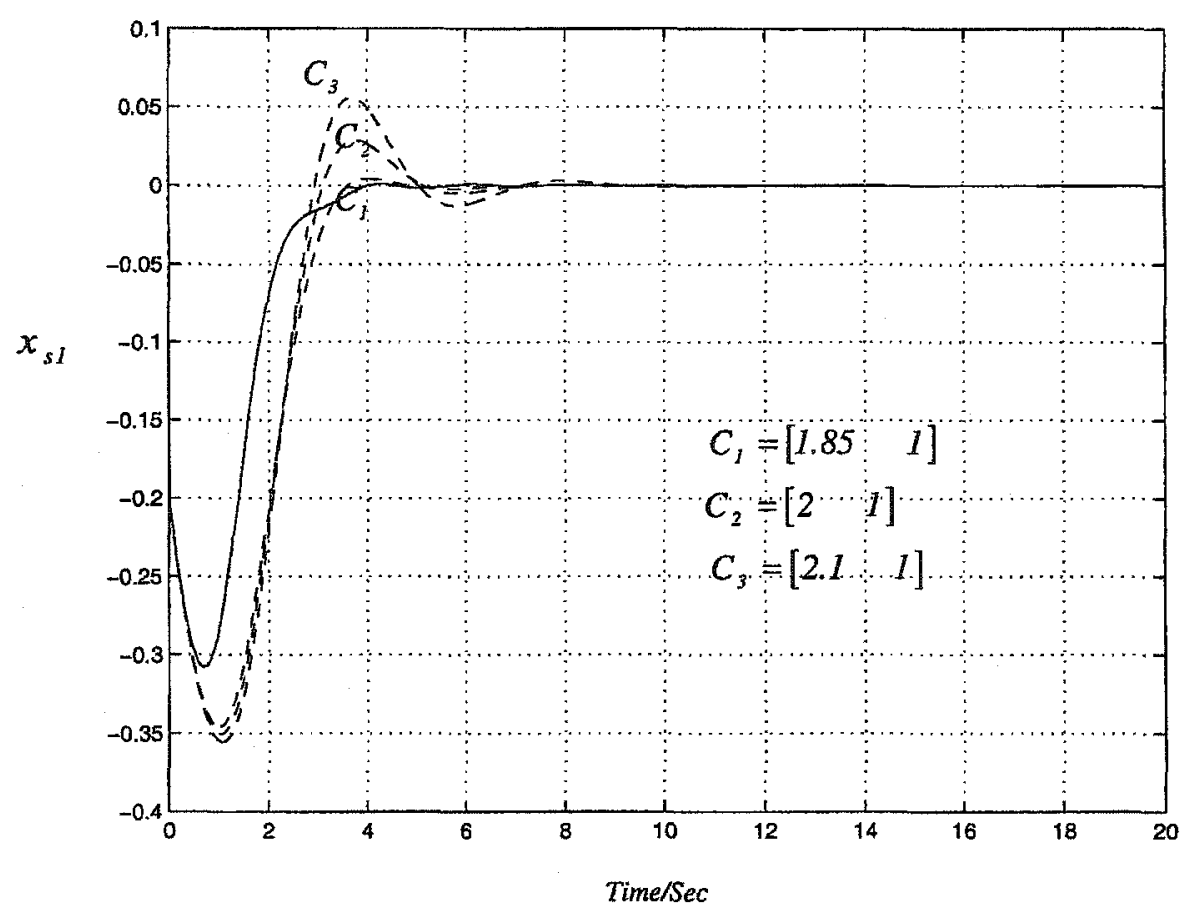

Figure 6.13: Closed loop time response of slow state for different sliding surface

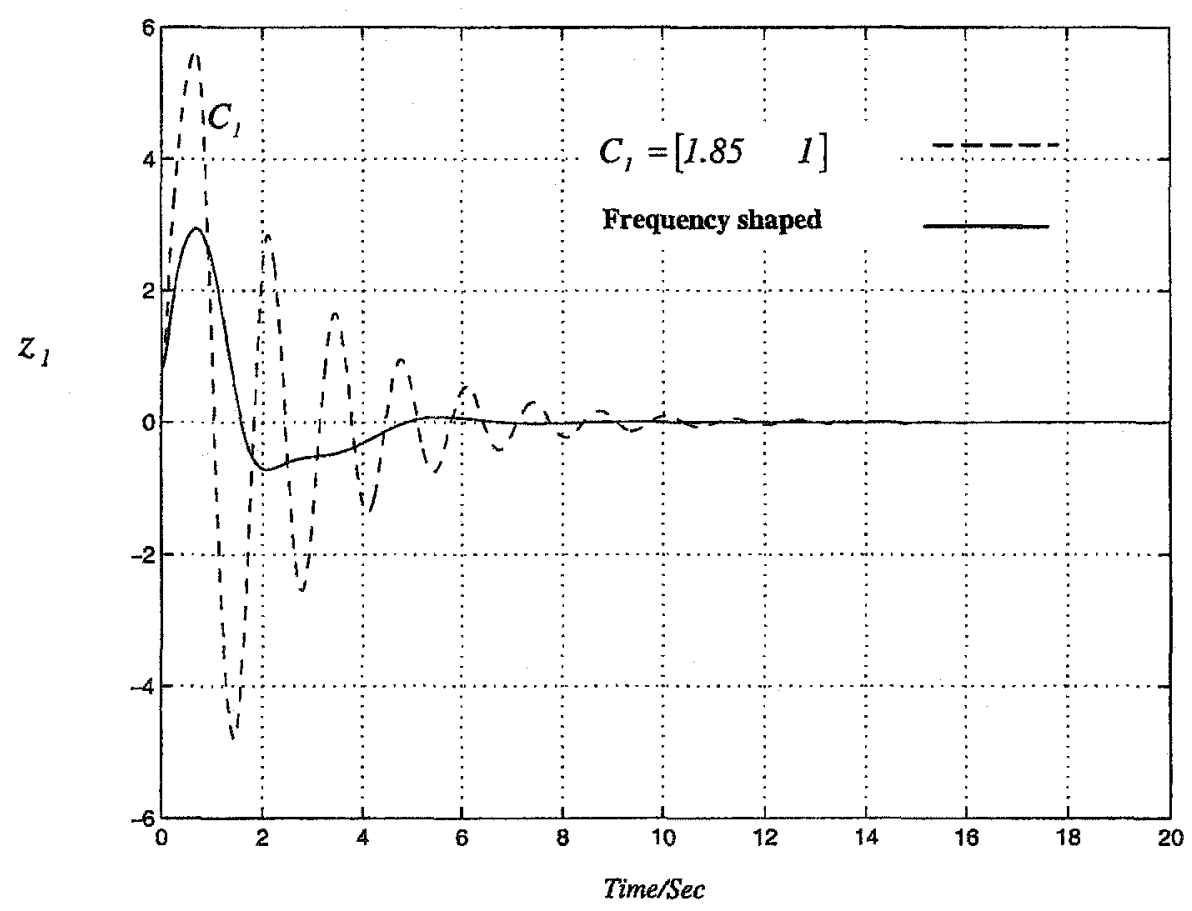

Figure 6.14: Closed loop time response of fast states $z_{1}$ 


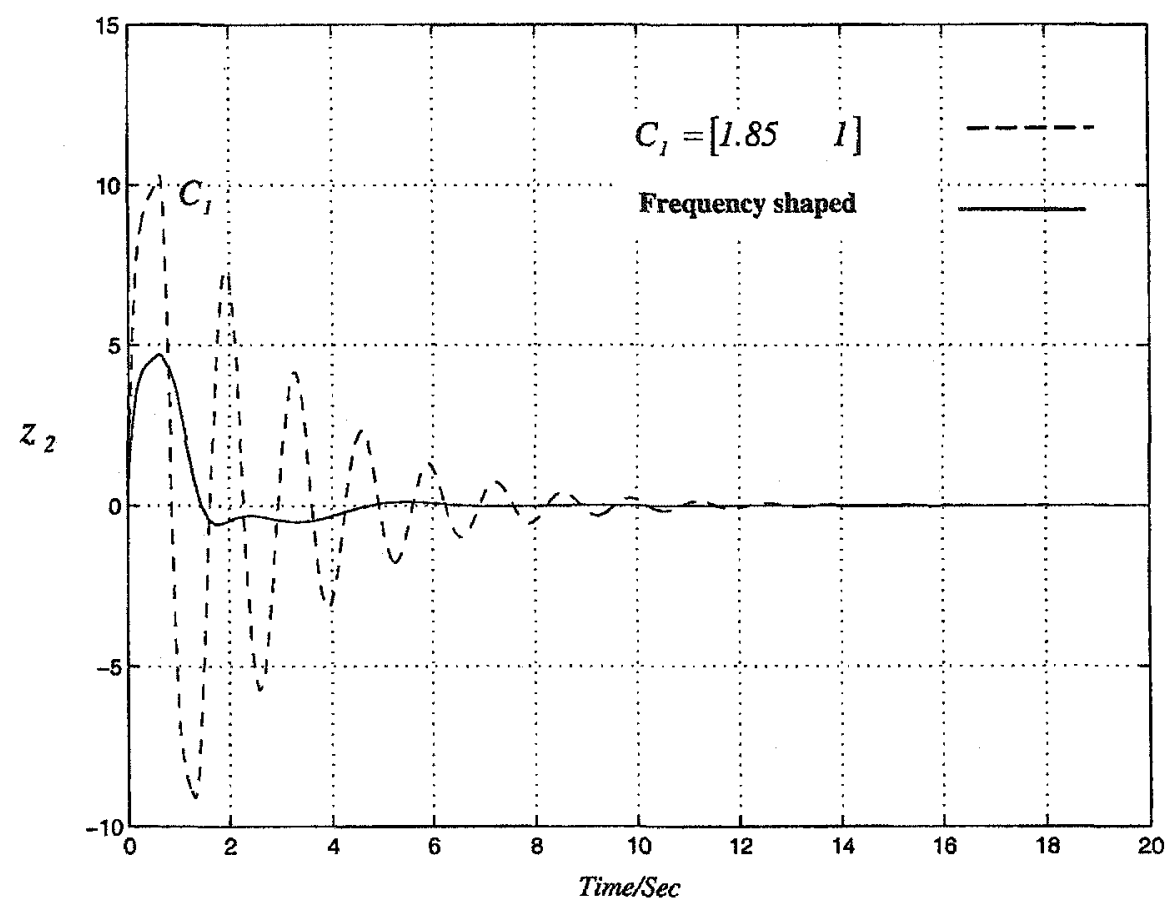

Figure 6.15: Closed loop time response of $z_{2}$

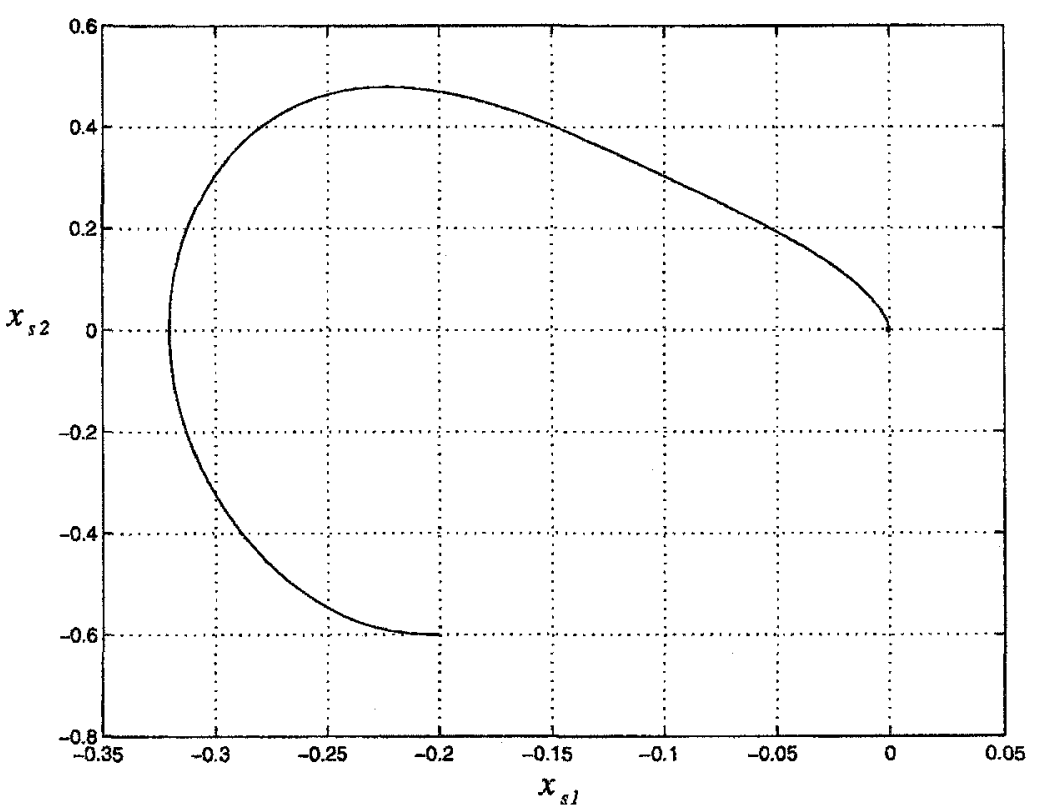

Figure 6.16: Phase plane plot for frequency shaped sliding mode controller 


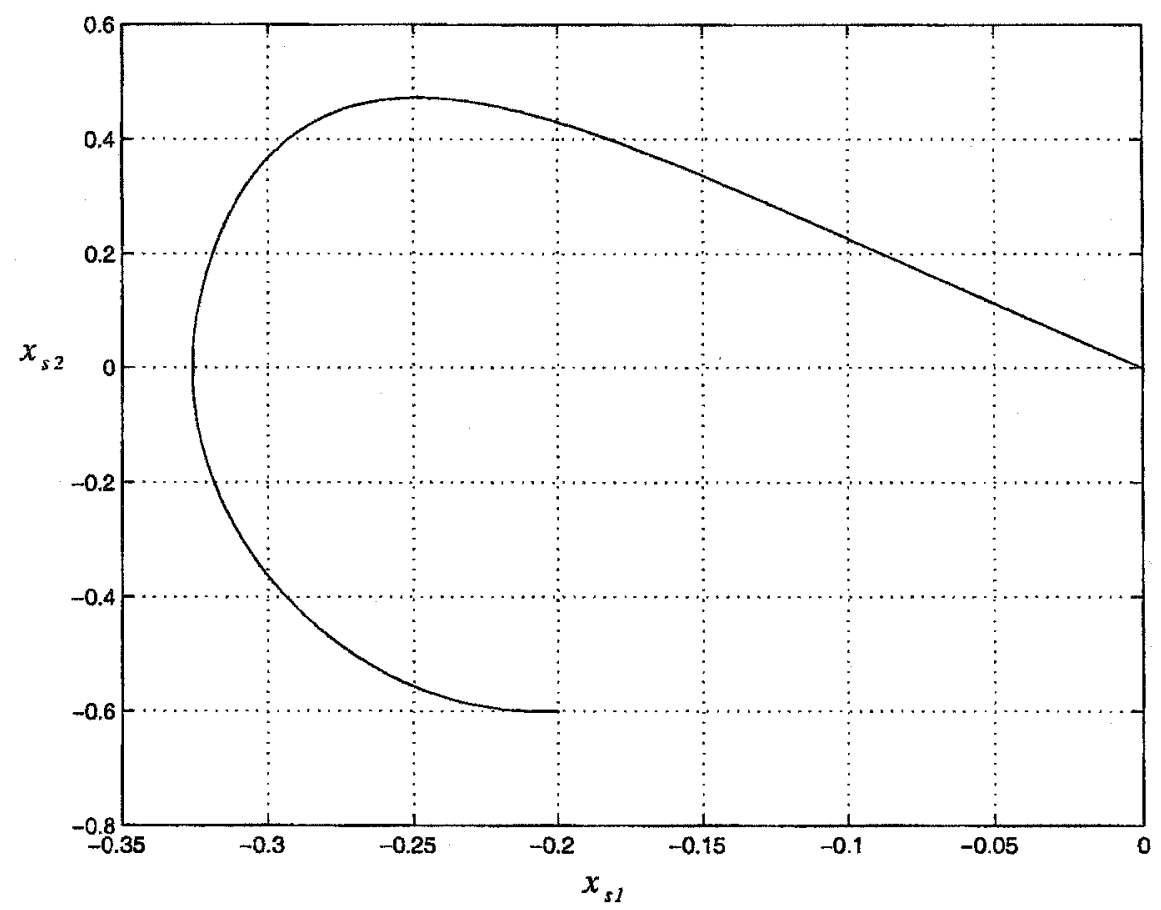

Figure 6.17: Phase plane plot for sliding mode controller with $C_{s}=\left[\begin{array}{ll}1.85 & l\end{array}\right]$ 


\section{Chapter 7 Fuzzy Sliding Mode Control For a Singularly Perturbed System}

\subsection{Introduction}

In this chapter we will design a fuzzy sliding mode controller for reduced singularly perturbed systems.

Fuzzy logic was first introduced by Zadeh [64]. His work was to extend the crisp set which has only yes or no as an answer to the belonging question, to a fuzzy set which has a degree of belonging.

The idea can be simply explained by Figure 7.1.a. Clearly point " $a$ " is a member of set $A$ which is located in the universe $X$ while point "b" is not a member of set $A$. In Figure 7.1.b, A is a fuzzy set and the shaded boundary describes the fuzzy boundary of region $A$. If "a" is clearly a member of $A$ and " $b$ " is not a member of $A$, and if complete membership is represented by a number " 1 " and no membership is represent by " 0 "then point "c" has a degree of membership, which is neither "1" nor "0" but some value between them. As a result, fuzzy sets can be defined as a set that contains elements that have a varying degree of membership in the set.

This chapter is organized as follows: Fuzzy logic theory is reviewed in section 7.1. Section 7.2 describes the fuzzy sliding mode control concept. Section 7.3 illustrates the design of fuzzy sliding mode control for the singularly perturbed system. 


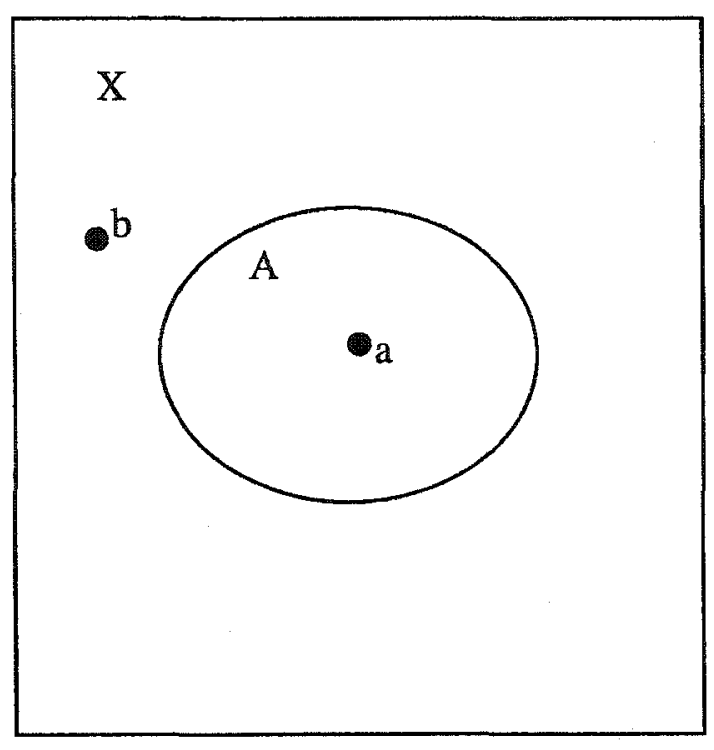

a

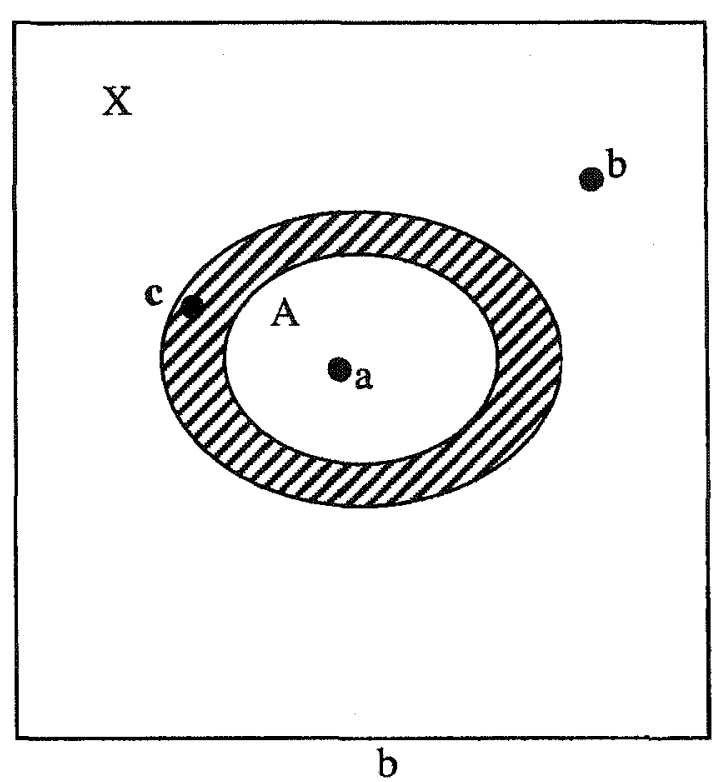

Figure 7.1: Classical and Fuzzy sets

The components of fuzzy systems are quite similar in each system, Lee [33][34]. The two primary components are, "fuzzifiers" which map crisp inputs to their fuzzy representative values and "defuzzifiers", which do the opposite, they map the output of the fuzzy process to a crisp value. In fuzzy systems the value of the fuzzified input executes all of the rules in the knowledge repository which will generate a new fuzzy set representing each output or solution variable.

Defuzzification creates a value of the output variable from the new fuzzy set. For a physical system the output value is often used to adjust the setting of the actuator that in turn adjusts the state of the physical system. The sensor picks up the change and the entire process starts again.

The basic configuration of a fuzzy logic controller consists of four main steps [33]. This can be explained by Figure 7.2, which has the following components: 
1. Knowledge base:

Contains knowledge of the application and it consists of two parts, "Data base" and "fuzzy control rule base", which will be explained in detail in the next section.

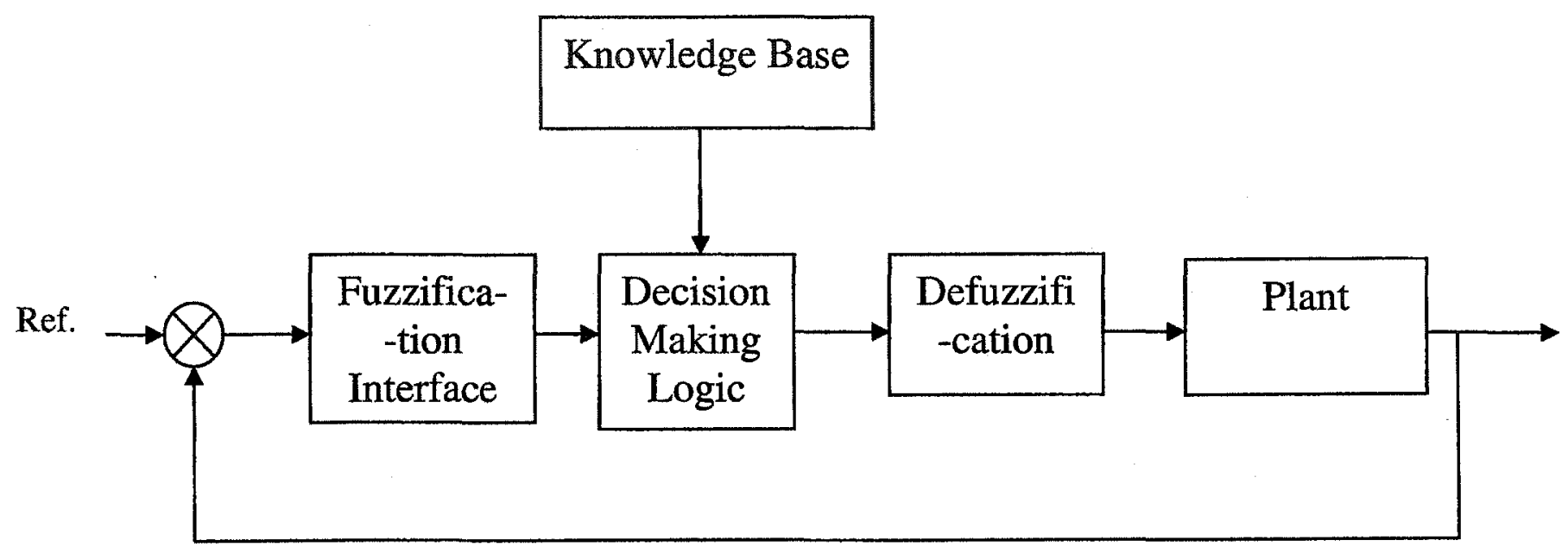

Figure 7.2: Basic configuration of fuzzy logic controller [33].

\section{Fuzzification interface}

In this section the crisp input is measured and is assigned to certain fuzzy values (may be more than one) based on fuzzy sets of the input.

\section{Decision making logic}

This is the main part of the fuzzy logic controller. It has the capability of simulating human thinking based on certain fuzzy algorithms (inference engine). Different fuzzy algorithms (inference engine) are discussed in the next sections. 


\section{Defuzzification}

In this part the output of the inference process that is still a fuzzy value is changed to a crisp control value. There are many possible methods for defuzzification and the choice between them depends on the fuzzy inference method and system requirements.

It is important to have a good design methodology for every component of the fuzzy systems. There are many methods for the design of fuzzy logic controllers; details can be found in Lee [33][34].

\subsection{Sliding mode fuzzy logic control (SMFLC)}

Lately fuzzy logic has been used to improve the characteristic of sliding mode control. Many articles have been published on this subject [2][27][35][59]. Two main approaches were found in these articles. First, fuzzy logic has been used to overcome the chattering problem without sacrificing the robustness of the sliding mode controller. This is done by changing the value of the discontinuous control in the sliding mode control law based on the location of the state from the sliding surface. The main advantage for this method is that we get a chattering free sliding mode controller and the robustness properties are preserved [42][59]. Second, fuzzy logic is used to connect different sliding mode controllers together. This will give a nonlinear switching surface and also a variable thickness boundary layer. The details of these two methods can be found in many articles $[35][38][43]$. 


\subsection{Fuzzy sliding mode control for singularly perturbed}

\section{systems}

The problem, as has been described before, is to avoid the excitation of the high frequency dynamics. This can be done by making appropriate choices of the sliding surface parameters. It is desired that the surface parameters satisfy two conflicting objectives, one is a fast closed loop response, and the second is a stable closed loop system without exciting the high frequency dynamics.

Lately, fuzzy logic has been used to obtain a nonlinear sliding surface. A number of articles with different approaches have been published on this topic [27][35][36][38]. We propose, in this section, to design a single fuzzy sliding mode controller using the supervisory method [2]. This controller has a piecewise linear switching surface and is designed based on the slow subsystem only to control the full order singularly perturbed system.

The system considered in this section may be represented in the usual form,

$$
\begin{aligned}
& \dot{x}=A_{11} x+A_{12} z+B_{1} u, \\
& \varepsilon \dot{z}=A_{21} x+A_{22} z+B_{2} u,
\end{aligned}
$$

where $x \in R^{n}, z \in R^{m}, u \in R^{r}$ and $\varepsilon$ is a small positive parameter. This is a form for standard linear time invariant singularly perturbed systems. Recall the quasi steady state method to obtain the reduced order subsystem as described in chapter 3 section 3.2 . By setting the perturbation parameter $\varepsilon$ to zero then equation (7.1) and equation (7.2) can be written as,

$$
\dot{\tilde{x}}=A_{I I} \tilde{x}+A_{12} \tilde{z}+B_{l} \tilde{u}
$$


$0=A_{2 I} \tilde{x}+A_{22} \tilde{z}+B_{2} \tilde{u}$

where the symbol " $\sim$ " indicates that $\varepsilon=0$. Thus we can get the following reduced system [30],

$\dot{x}_{s}=A_{0} x_{s}+B_{0} u$

where $A_{0}=A_{11}-A_{12} A_{22}^{-1} A_{21}$ and $B_{0}=B_{1}-A_{12} A_{22}^{-1} B_{2}$.

Assuming that the slow variables are constant during fast transients i.e. $\dot{\tilde{z}}=0$ and $\tilde{\boldsymbol{x}}=\boldsymbol{x}_{s}=$ constant, the approximated fast subsystem for the system given by equations (7.1) and (7.2) can be defined as,

$\varepsilon \dot{x_{f}}=A_{22} x_{f}+B_{2} u_{f}$

where $\boldsymbol{x}_{f}=\boldsymbol{x}-\tilde{\boldsymbol{x}}$ and $u_{f}=u-\tilde{u}$.

The slow sliding mode controller is designed using the slow reduced order model given by equation (7.5). The equivalent control method [10][55] is used to design such a controller. We define the linear switching surface as $S_{s}=C \boldsymbol{x}_{s}$. The control law, as discussed before in chapter 2 , has the form,

$$
u=-\left[\left(C B_{0}\right)^{-1} C\right] A_{0} x_{s}-\left(C B_{0}\right)^{-1} \eta_{I} \operatorname{sgn}\left(S_{s}\right)(7.8)
$$

Using this control law we will design two controllers with different surface parameters for the slow subsystem given by equation (7.5). Fuzzy logic is used to connect these two controllers in an appropriate way. This is discussed in the next subsection

\subsubsection{Fuzzy sliding mode controller}

The performance of a system under sliding mode control is sensitive to sliding surface parameter $C$. A "large value" of $C$ is desirable since this decreases the overall 
settling time. However, by increasing $C$, the sliding mode domain of attraction shrinks and causes the closed loop system to be sensitive to high frequency dynamics that can result in high chattering or instability. On the other hand, if "small values" of $C$ are chosen, the convergence speed to the equilibrium point will be slow leading to a longer settling time but the system tends not to excite the high frequency dynamics and instability can be avoided.

Kalaykov [28], design a sliding surface to obtain the best performance in the time response point of view. He combined the time optimal control method with the sliding mode control algorithm to obtain a nonlinear sliding surface that will satisfy the time response requirement and at the same time preserve robustness. The sliding surface obtained from this approach has the form shown in Figure 7.3.

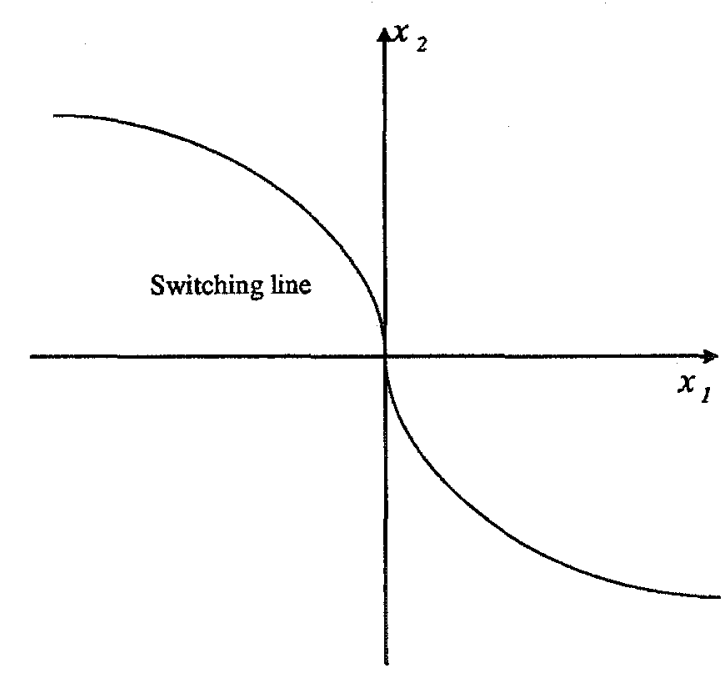

Figure 7.3: Time optimal control switching surface [28]

In this section, considering the shape of sliding surface obtained by Kalaykov [28], two sliding mode controllers are separately designed to obtain especially good characteristics in the state where each one provides the required control actions for the specified area in the phase plane. These controllers will be defined as, primary sliding mode controllers with "small values" of $C$ and terminal sliding mode controllers with 
"large values" of $C$. For the primary sliding mode controller the control law will have the form,

$$
\begin{aligned}
u_{P}= & u_{e q P}+u_{N P} \\
& =-\left[\left(C_{P} B_{0}\right)^{-1} C_{P}\right] A_{0} x_{s}-\left(C_{P} B_{0}\right)^{-1} \eta_{I} \operatorname{sgn}\left(S_{P}\right)
\end{aligned}
$$

while the control form for the terminal sliding mode controller can be written as

$$
\begin{aligned}
u_{T} & =u_{e q T}+u_{N T} \\
& =-\left[\left(C_{T} B_{0}\right)^{-1} C_{T}\right] A_{0} x_{s}-\left(C_{T} B_{0}\right)^{-1} \eta_{l} \operatorname{sgn}\left(S_{T}\right)
\end{aligned}
$$

where for a second order slow subsystem, the slope of the sliding surface for the terminal sliding mode controller is more steep than the slope of sliding surface for the primary sliding mode controller.

Several design approaches for the fuzzy sliding mode controllers can be found [2] [65][58] to obtain a nonlinear switching surface. The supervisor controller method [2] is used in this chapter where the fuzzy controller is used as a supervisory system in a higher hierarchical level. The main objective for the fuzzy logic controller in this case is to change the $\alpha$ value as shown in Figure 7.4 where $\alpha \in[0,1]$. When the states $x_{1}$ and $x_{2}$ are small the terminal sliding mode control becomes dominant $(\alpha=0)$. Conversely, when the states are large then the primary sliding mode controller is dominant $(\alpha=1)$.

The fuzzy controller used is the Sugeno type [53] with singleton consequence. The rules will be arranged based on the lookup table shown in Figure 7.5. The inference algorithm for the fuzzy controller can be seen in Figure 7.5 where $(Z)$ stands for zero, (M) for medium and (L) for large. 


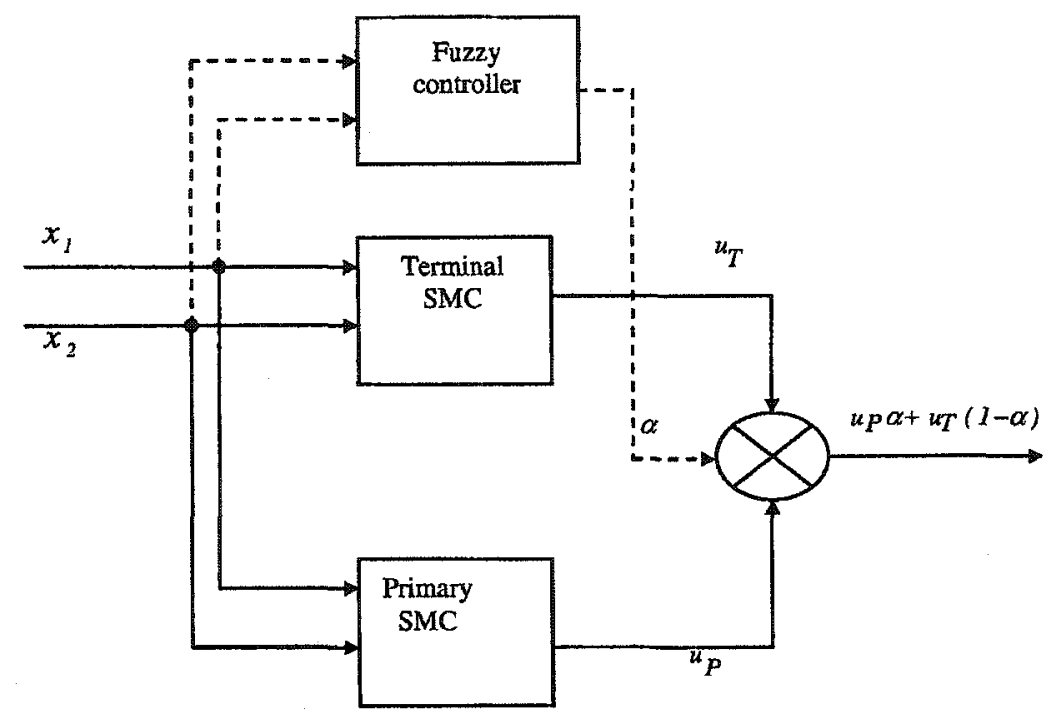

Figure 7.4: Fuzzy Sliding mode controller

The output of fuzzy logic control $(\alpha)$ is used to determine the control law $u$, which will control the plant and takes the form [16],

$$
u=u_{P} \alpha+u_{T}(1-\alpha)
$$

The simulation results for the single fuzzy sliding mode controller shows superior performance compared with the linear surface sliding mode controller.

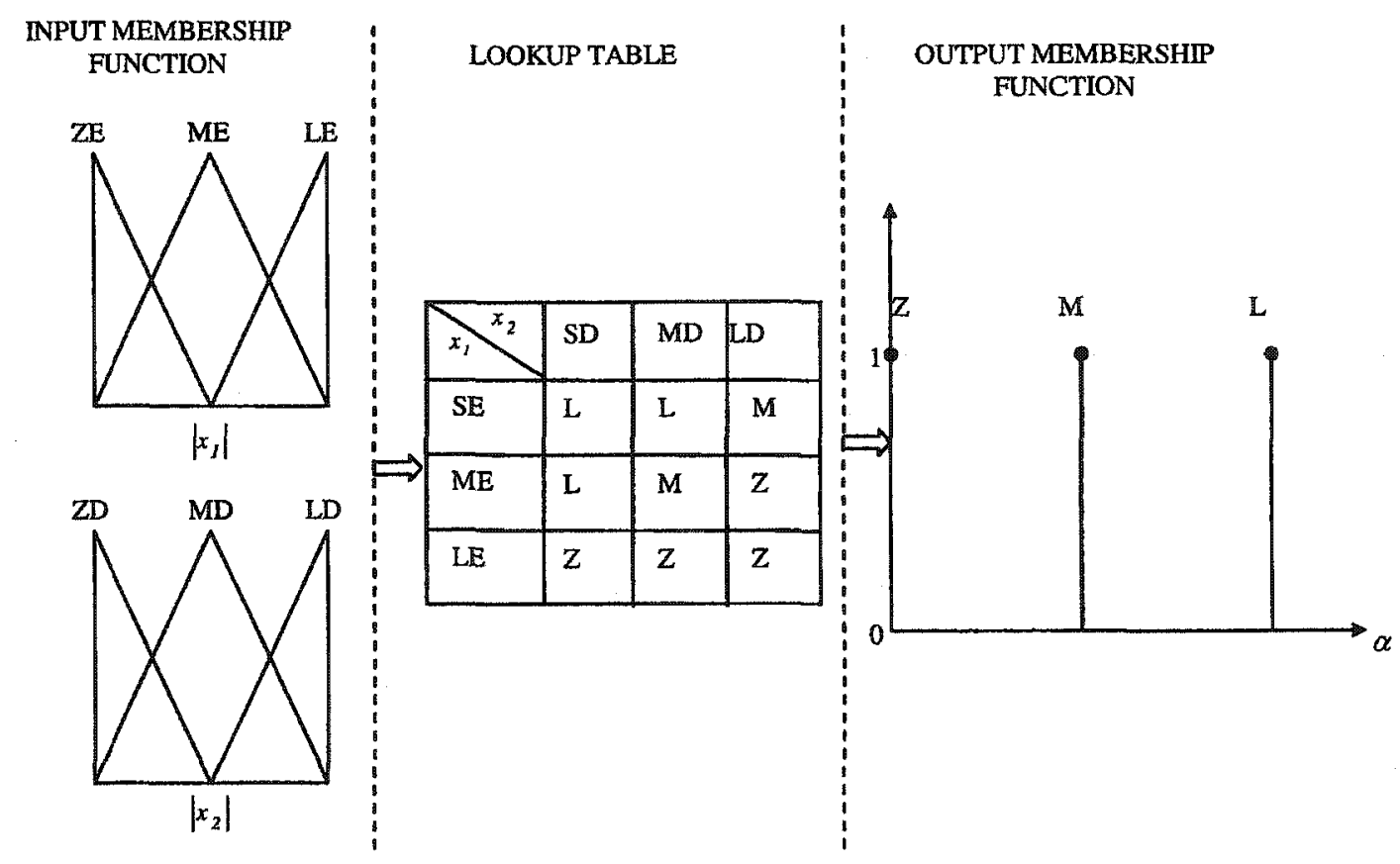

Figure7.5: Supervisory fuzzy logic controller [16] 


\subsubsection{Illustrative example}

Consider a magnetic tape control system given by

$$
\begin{aligned}
& {\left[\begin{array}{l}
\dot{x}_{1} \\
\dot{x}_{2} \\
\varepsilon \dot{z}_{1} \\
\varepsilon \dot{z}_{2}
\end{array}\right]=\left[\begin{array}{cccc}
0 & 0.4 & 0 & 0 \\
0 & 0 & 0.345 & 0 \\
0 & -0.524 & -0.465 & 0.262 \\
0 & 0 & 0 & -1
\end{array}\right]\left[\begin{array}{l}
x_{1} \\
x_{2} \\
z_{1} \\
z_{2}
\end{array}\right]+\left[\begin{array}{l}
0 \\
0 \\
0 \\
1
\end{array}\right] u} \\
& y=\left[\begin{array}{llll}
1 & 0 & 0 & 0 \\
0 & 0 & 1 & 0
\end{array}\right]\left[\begin{array}{l}
x_{1} \\
x_{2} \\
z_{1} \\
z_{2}
\end{array}\right] .
\end{aligned}
$$

This can be put in standard singularly perturbed form as,

$$
\begin{aligned}
& \dot{x}=A_{11} x+A_{12} z+B_{1} u, \\
& \varepsilon \dot{z}=A_{12} x+A_{22} z+B_{2} u,
\end{aligned}
$$

where $\quad A_{11}=\left[\begin{array}{cc}0 & 0.4 \\ 0 & 0\end{array}\right], \quad A_{12}=\left[\begin{array}{cc}0 & 0 \\ 0.345 & 0\end{array}\right], \quad A_{12}=\left[\begin{array}{cc}0 & -0.524 \\ 0 & 0\end{array}\right]$,

$$
\begin{aligned}
& A_{22}=\left[\begin{array}{cc}
-0.465 & 0.262 \\
0 & -1
\end{array}\right] \\
& B_{1}=\left[\begin{array}{l}
0 \\
0
\end{array}\right], B_{2}=\left[\begin{array}{l}
0 \\
1
\end{array}\right] \text { and } \varepsilon=0.1 .
\end{aligned}
$$

Setting $\varepsilon=0$ in equation (7.14) gives us the reduced slow subsystem

$$
\dot{x}_{s}=A_{0} x_{s}+B_{0} u,
$$

where $A_{0}=A_{11}-A_{12} A_{22}^{-1} A_{21}=\left[\begin{array}{cc}0 & 0.4 \\ 0 & -0.38\end{array}\right]$ and $B_{0}=B_{1}-A_{12} A_{22}^{-1} B_{2}=\left[\begin{array}{c}0 \\ 0.1944\end{array}\right]$

The surface parameter for the primary sliding mode controller is chosen based on the equivalent control method [55]. The terminal sliding mode controller surface parameters 
are initially chosen based on Slotine's suggestion that the natural frequency of the system in the sliding mode is chosen to be smaller than the lowest frequency of the fast modes (high frequency modes). The surface parameters are then given by,

$$
\begin{aligned}
& C_{T}=\left[\begin{array}{ll}
4 & 1
\end{array}\right] \\
& C_{P}=\left[\begin{array}{ll}
2 & 1
\end{array}\right]
\end{aligned}
$$

where $C_{T}$ and $C_{P}$ are the surface parameters for the terminal and primary sliding mode controllers respectively. The value of $\alpha$ that is determined by the fuzzy logic controller can be calculated as illustrated in Figure 7.6.

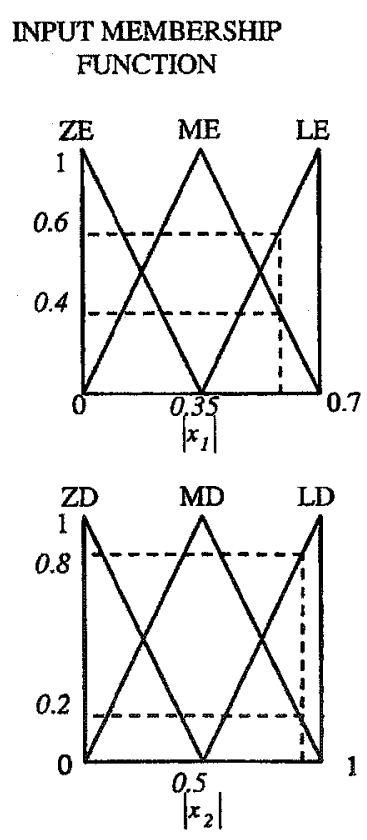

OUTPUT MEMBERSHIP FUNCTION
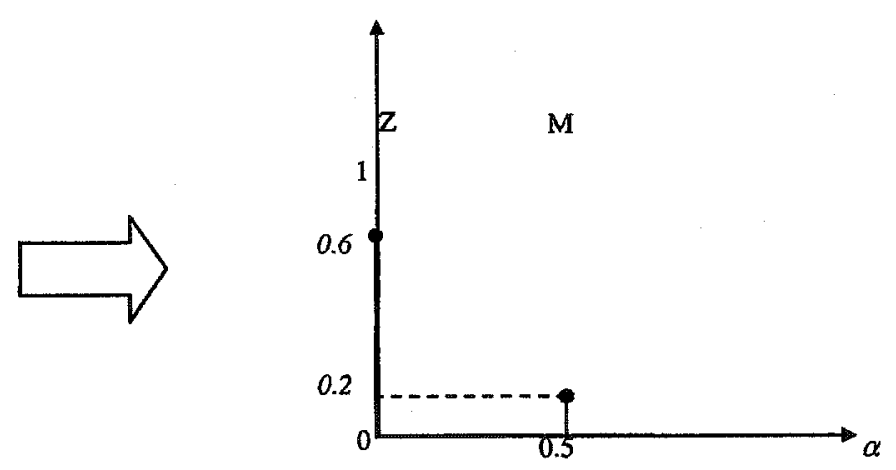

Figure 7.6: Inference algorithm for $\alpha$

Consider the case of $x_{1}$ and $x_{2}$ that have the following values, $x_{1}=0.5$ and $x_{2}=0.9$.

These two values activate four rules in the fuzzy lookup table as follows:

- IF $x_{I}$ "medium (0.4)" AND $x_{2}$ "medium (0.2)" THEN $\alpha$ is medium(0.2) 
- IF $x_{1}$ "medium (0.4)" AND $x_{2}$ "large(0.8)" THEN $\alpha$ is zero (0.4)

- IF $x_{1}$ "large (0.6)" AND $x_{2}$ "medium (0.2)" THEN $\alpha$ is zero (0.2)

- IF $x_{1}$ "large (0.6)" AND $x_{2}$ "large (0.8)" THEN $\alpha$ is zero (0.6)

Based on the Min-Max method the output membership function will be medium (0.2) and zero (0.6), as shown in Figure 7.6. The output of the fuzzy logic controller will be calculated using the center of gravity method as,

$$
\alpha=\frac{0.6 \times 0+0.2 \times 0.5}{0.6+0.2}=0.125
$$

Figure 7.7 and Figure 7.8 illustrate the closed loop time response for the slow subsystem. The time response of sliding mode controller designed using the equivalent control method has been shown for two different sliding surfaces.

The initial condition is given by,

$$
\left[\begin{array}{l}
x_{s 1}(0) \\
x_{s 2}(0)
\end{array}\right]=\left[\begin{array}{l}
0.3 \\
0.4
\end{array}\right],\left[\begin{array}{l}
x_{f 1}(0) \\
x_{f 2}(0)
\end{array}\right]=\left[\begin{array}{l}
0 \\
0
\end{array}\right] \text { and the boundary layer } \phi=0.05
$$

It is shown that the fuzzy sliding mode controller has superior performance when compared with the linear sliding surface controller obtained by equivalent control method with control law given by equation (7.8). Figure 7.9 shows the unstable result that we obtain when steep slope is chosen for the single sliding mode control. Figure 7.10 shows the changing value of $\alpha$.

Nevertheless, the result from the fuzzy logic controller can be comparable to nonlinear sliding surface methods. In fact frequency shaped sliding surfaces can have better performance. Figure 7.11 illustrates the closed loop time response of the full order system when using $H^{\infty}$ frequency shaped sliding mode controller and fuzzy sliding 
mode controller. $H^{\infty}$ frequency shaped sliding mode controller has similar performance to fuzzy sliding mode controller in the slow modes. However $H^{\infty}$ is more effective in suppressing the high frequency dynamics than fuzzy sliding mode controller. This is shown in Figure 7.11.b and Figure 7.11.c where the closed loop time response of fast states is investigated.

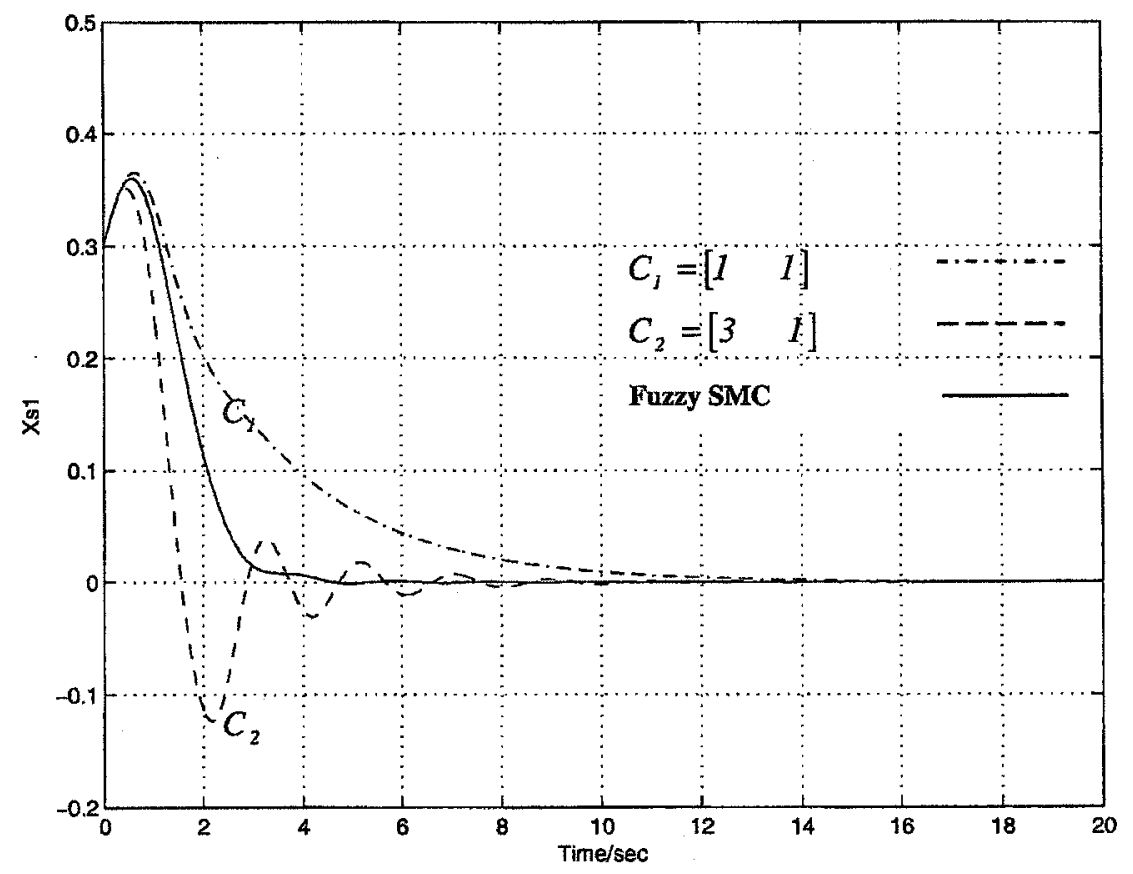

Figure 7.7: Closed loop time response of $\boldsymbol{x}$ 


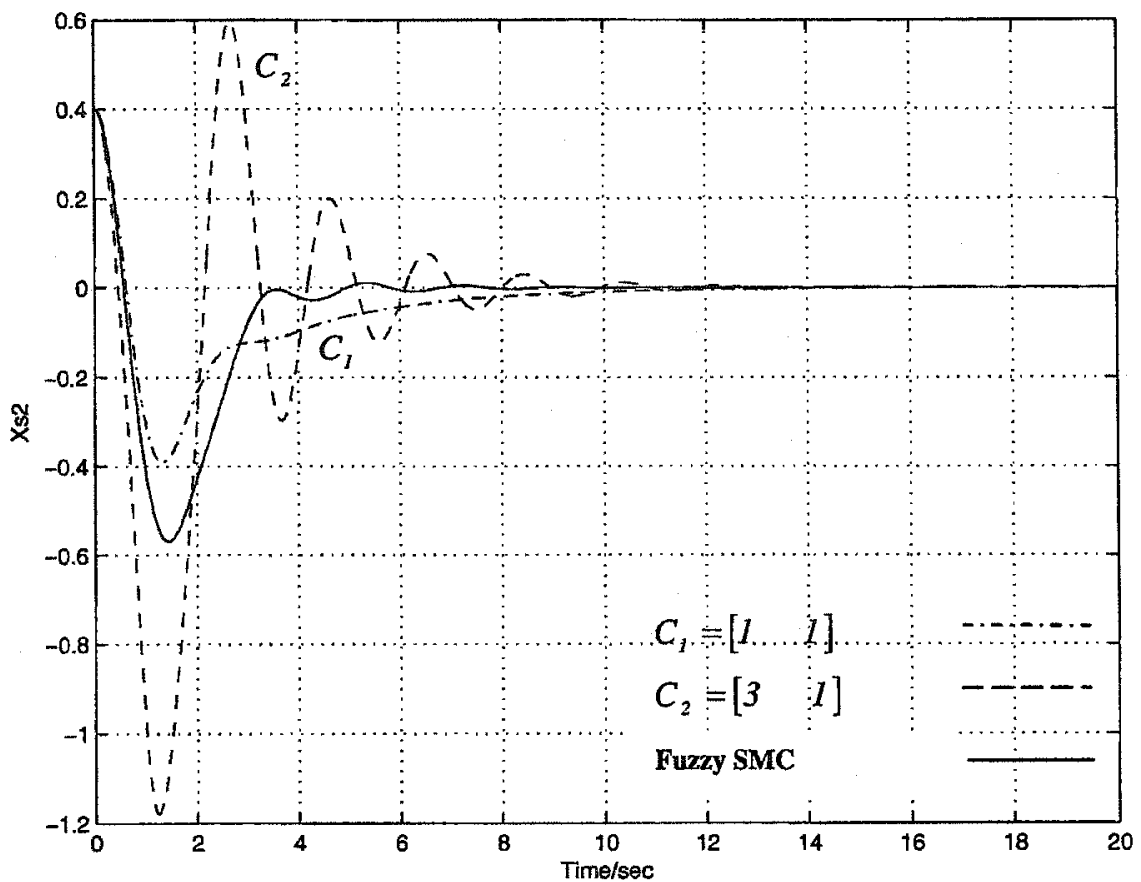

Figure 7.8: Closed loop time response of $\boldsymbol{x}$

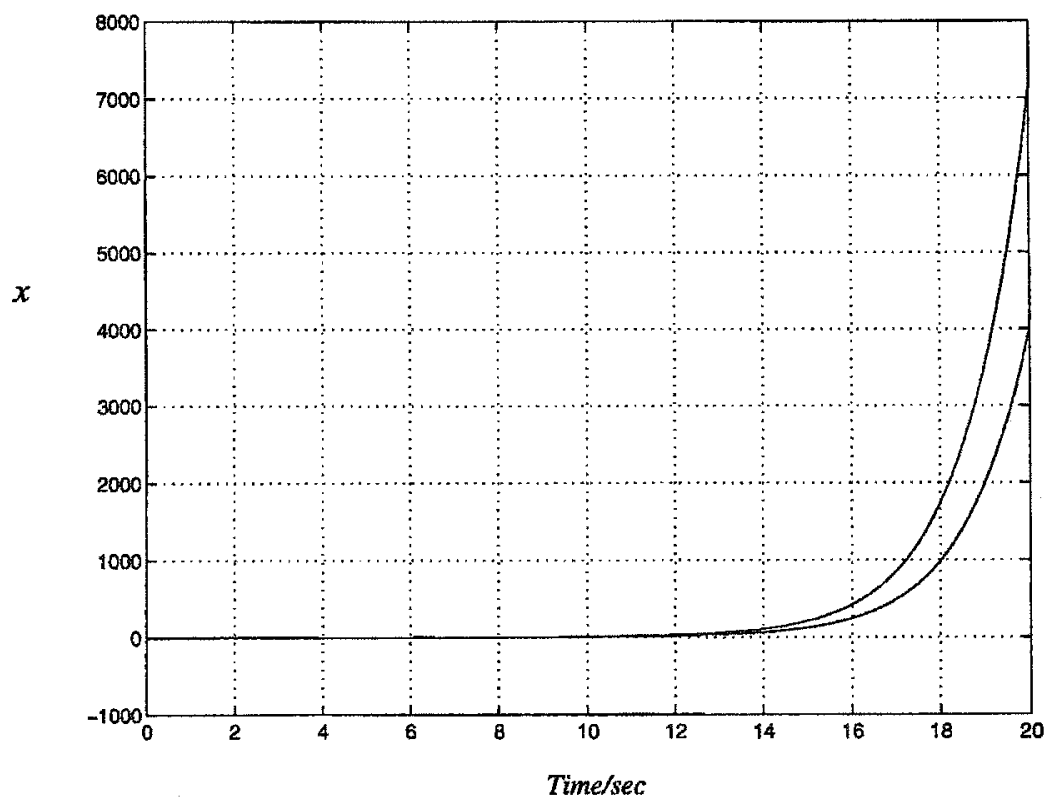

Figure 7.9: Closed loop time response of $\boldsymbol{x}$ (Slotine approach) 


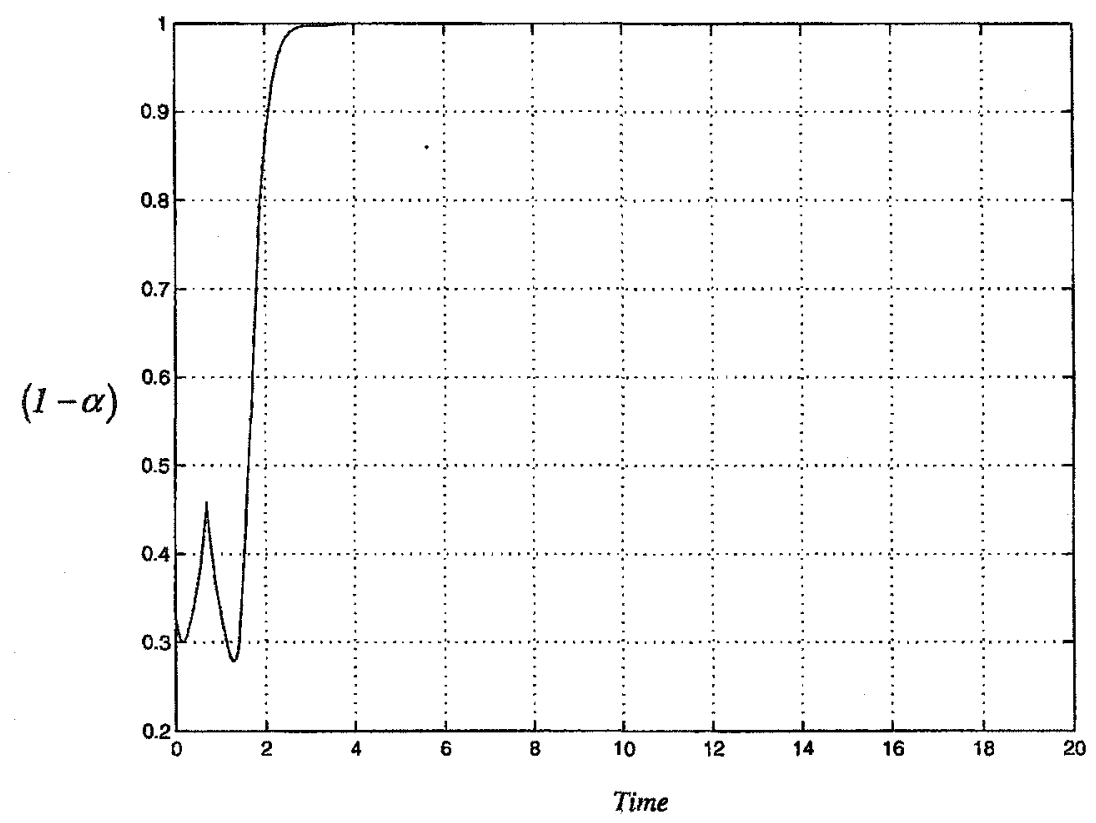

Figure 7.10: Time response of $(1-\alpha)$

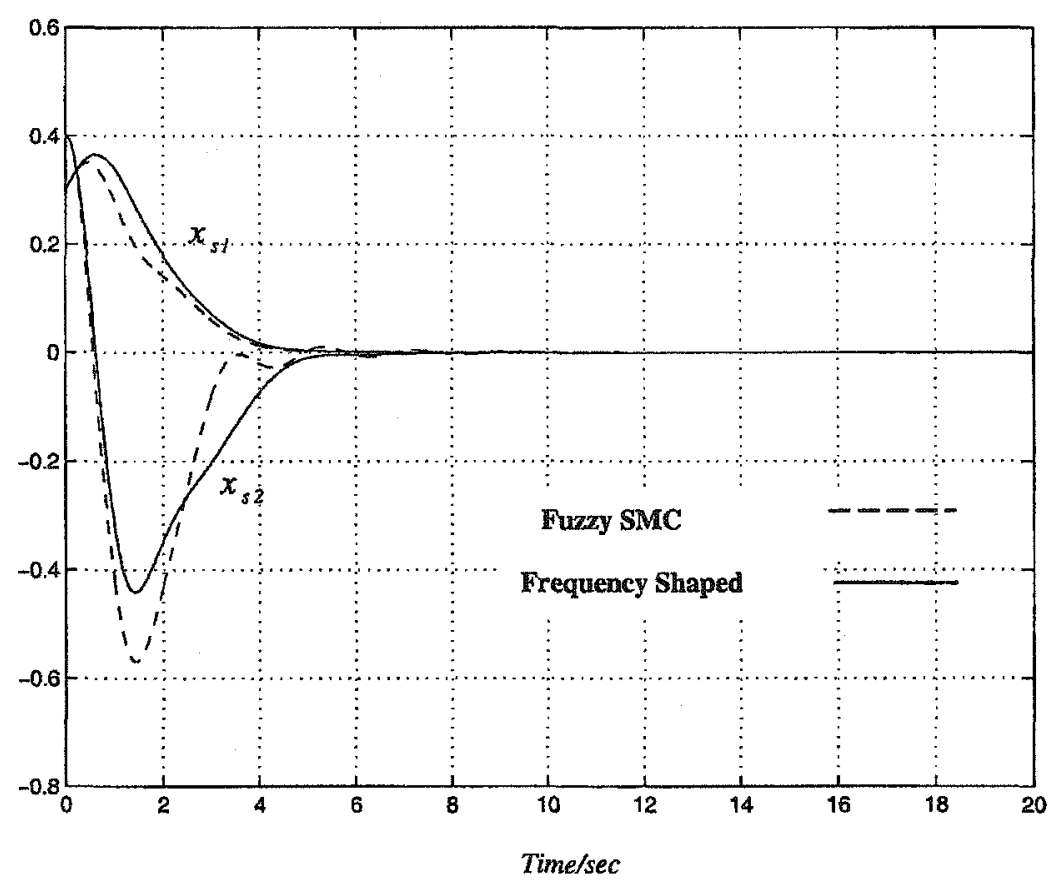

Figure 7.11.a: Closed loop time response of $x$ (slow states) 


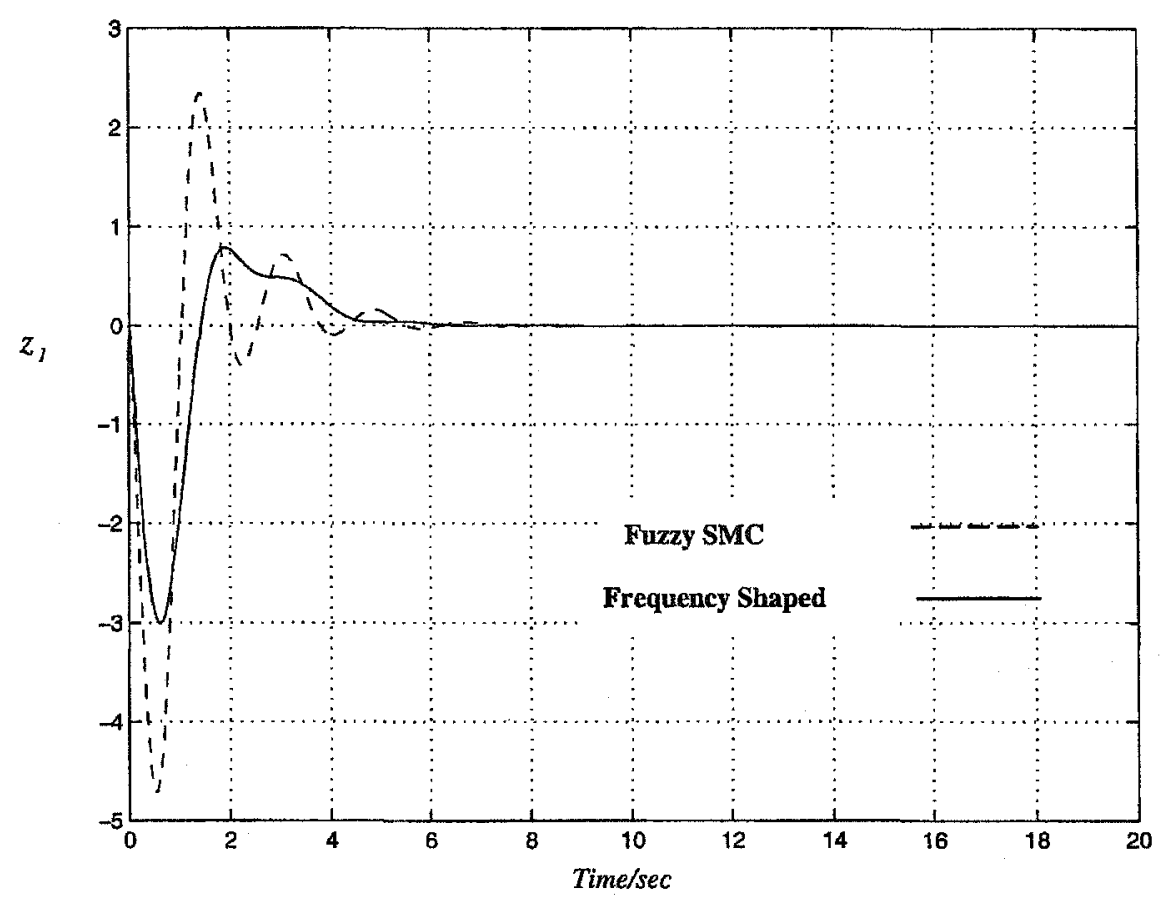

Figure 7.11.b: Closed loop time response of $z_{1}$

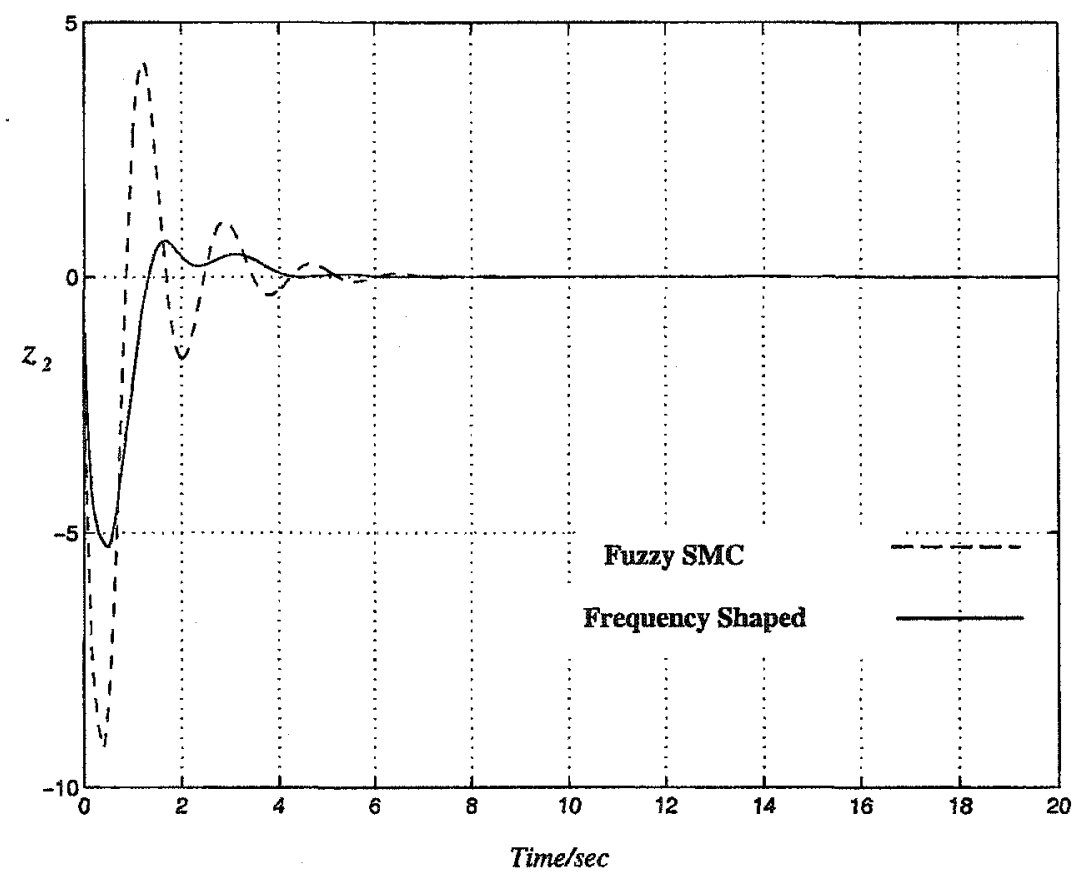

Figure 7.11.c: Closed loop time response of $z_{2}$ 


\section{Chapter 8 Conclusions and Future Work}

\subsection{Discussion and conclusions}

This thesis addresses the use of sliding mode control for singularly perturbed systems.

The singular perturbation method provides us with a powerful tool for investigating a system that has high and low frequency components. An important aspect of analyzing systems using the singular perturbation method lies in the fact that singular perturbation theory separates the full order system into fast and slow subsystems. Chapter 3 provided an overview of the singular perturbation method and the methods used to obtain the reduced order subsystem from the full order model.

This thesis provides a new approach to control the full order singularly perturbed model using a sliding mode controller. A single sliding mode controller is used to control the full order singularly perturbed model. The simulation results show that it is possible to control the full order model with a single sliding mode controller based on the slow subsystem while taking into account the fast subsystem in the design process.

The benefits from this approach are that, the control effort needed to obtain the same response is greatly reduced. This is an important advantage especially if we use actuators with limited capacity (i.e. saturating actuators). The controller needs only the slow states for feedback. As a result, the fast state does not have to be measured which is usually a difficult task. We showed that using the diagonalization method [30] to decouple the singularly perturbed model could avoid the approximation errors that usually come with 
the quasi steady state method. The main two factors affecting the stability of the proposed controller are the selection of surface parameters in the sliding mode controller and the perturbed parameter in the singularly perturbed model.

The stability analysis for a single sliding mode controller used to control the full order singularly perturbed system was investigated. We found the maximum perturbed parameter $\varepsilon^{*}$ that will ensure the stability of the closed loop system for a given surface parameter. In this case the condition we proved in theorem 1 in chapter 5 is sufficient but not necessary. The simulation results showed that for a computed value of $\varepsilon^{*}$ the closed loop system may maintain stability for a higher value of $\varepsilon$. This was because the theoretical result was a conservative condition. Other methods for stability analysis of a singularly perturbed model can be found in the literature. These methods include Nyquist stability analysis [5][18] and state space based stability analysis [7]. These methods may obtain improved boundaries for maximum perturbation parameters. This may be a good approach for future work.

The maximum perturbation parameter $\varepsilon^{*}$ obtained from the stability analysis is for given surface parameters. Choosing the surface parameters is a difficult task as discussed in chapter 6 . We introduced the frequency shaped method to obtain a nonlinear switching surface.

The $H^{\infty}$ frequency shaped sliding mode control method was investigated and used for the proposed single sliding mode controller. The main idea is to penalize the high frequency components of the control and reward the low frequency components. This has the advantage of aiding the designer of the sliding surface such that the neglected or high frequency states of the singularly perturbed system remain stable. The simulation results 
from this approach showed improved performance compared with other common design methods that are used for sliding mode control design such as the equivalent control method.

There are two advantages of using the $H^{\infty}$ approach to the sliding surface design. The first is that the problem of choosing the surface parameter is helped by knowledge of the high frequency dynamics and the second is related to the $H^{\infty}$ frequency shaped method which is associated with the selection of weighting function. Many articles [32][52] discuss the difficulty of choosing the weighting functions when using the $H^{\infty}$ frequency shaped design method. It usually takes many iterations to reach the desired performance. In our case, for singularly perturbed systems, the weighting function can be selected with much fewer design iterations. The weighting function is designed based on the fast subsystem. This was done by finding the $H^{\infty}$ norm for the fast subsystem. The $H^{\infty}$ norm can be regarded as the largest possible amplification factor of the system's steady state response [63].

A fuzzy sliding mode control technique is also investigated. One of the advantages found in this area is that fuzzy logic could provide us with a piecewise linear sliding surface. This means different surfaces for different intervals. The interval in this case is defined based on an arbitrary parameter chosen by the designer. In the proposed controller the sliding surface selection was based on the fast states of the singularly perturbed system. This enabled us to obtain a nonlinear switching surface having similar results as those obtained with the $H^{\infty}$ frequency shaped sliding mode control. The properties introduced by fuzzy logic can be very useful if we use it to improve the performance of a sliding mode controller. The simulation results for the combined sliding surface showed improved 
performance when used with the proposed single sliding mode controller as shown in Figure 7.6 and Figure 7.7.

\subsection{Summary of contributions}

The main contributions of this thesis are now listed as:

1- A single sliding mode control has been developed for the full order singularly perturbed system. The benefits for using the proposed controller have been discussed. These benefits are:

a. Design of the single sliding mode controller for the full order singularly perturbed model provides insights into how the sliding mode control interacts with the high frequency dynamics. This is shown in several figures where fast states have been illustrated.

b. The control law in the proposed controller depends only on the slow state. As a result there is no need for measurement of the fast state, which is usually difficult to measure. The proposed controller seems to have less control efforts in the initial transient of the fast subsystem compared with the composite control method used before in literature.

2- An accurate decoupling method, which is called the diagonalization method, is used for composite and single sliding mode controller design. The errors that are produced during the decoupled process have been avoided. However, when a single sliding mode controller is designed for the slow subsystem obtained using the diagonalization method, the fast states must be measured. 
3- An interval for perturbation parameter $\varepsilon \in\left(O, \varepsilon^{*}\right)$ for the full order system using a single sliding mode controller is determined where the closed loop system is asymptotically stable. Furthermore, a relationship between the maximum perturbation parameter $\varepsilon^{*}$ and the sliding mode control surface parameters $C_{s}$ where the sliding surface has the form $S_{s}=C_{s} x_{s}$ and $x_{s}$ is the slow state, has been found. The relationship between $\varepsilon^{*}$ and $C_{s}$ is shown in Figure 5.12

4- An $H^{\infty}$ frequency shaped algorithm is proposed to a obtain a nonlinear switching surface for the single sliding mode controller. A weighting function selection procedure has been developed where the weighting function can be selected with less iteration procedure.

5- Fuzzy sliding mode control with a piecewise linear sliding surface was proposed. The results showed improved performance compared with common design methods for sliding mode controllers as shown in Figure 7.6 and Figure 7.7.

\subsection{Future research}

A number of directions are recommended for future research:

1- Expand the stability analysis for the single sliding mode controller for the full order singularly perturbed system to use other methods where we could find an improved maximum perturbation parameter limit.

2- In this thesis, a method for choosing the appropriate weighting function is shown. However, in choosing a weighting function, it will be useful to investigate in detail 
the type of filter that may be needed for a specific application to obtain improved performance.

3- All the algorithms in this thesis have been designed based on having full access to the system states (i.e. measurable states). This is not usually the case, thus designing an observer for the slow state would be another area that will be useful to investigate.

4- In this thesis the algorithms have been developed and numerical examples have been presented to verify the numerical analysis. Therefore, it is good to conduct experiments on the basis of these algorithms. 


\section{References}

[1] J. Alvarez-Gallegos, G. Silva-Navarro, "Two-time scale sliding mode control for a class of nonlinear systems," International Journal of Robust and Nonlinear Control, Vol. 7, 1997, pp. 865-879.

[2] F. Barrero, A. Gonzalez, A. Torralba, E. Galvan, L.G. Franquelo, "Speed control of induction motors using a novel fuzzy sliding mode structure," IEEE Transactions on Fuzzy Systems, Vol. 10, Issue. 3, Jun 2002, pp 375 -383.

[3] J. A. Burton, A. S. I. Zinober, "Continuous approximation of variable structure control," International Journal of Systems Science, Vol.17, 1986, pp. 875-885.

[4] L. Cao, H. M. Schwartz, "Output feedback stabilization of linear system with a singular perturbation model," Proceedings of the American Control Conference, 2002, pp. 1627-1632.

[5] L. Cao, H. M. Schwartz, "Complementary result on the stability bounds of singularly perturbed systems," Accepted for Publication IEEE Transactions on Automatic Control, May 2004

[6] P.C. Chandrasekharan, "Robust control of linear dynamical systems," Academic Press, 1996.

[7] B. S. Chen, Chun-Liang Lin, "On the stability bounds of singularly perturbed systems," IEEE Transactions on Automatic Control, Vol.35, 1990, pp. 1265-1270.

[8] S. B. Choi, D. W. Park and S. Jayasuriya, "A time varying sliding surfaces for fast tracking control of second order dynamic systems," Automatica, Vol. 30, 1994, pp.899-904. 
[9] J. H. Chow, P. V. Kokotovic, "A decomposition of near optimum regulators for system with slow and fast modes," IEEE Transactions on Automatic Control, 1976, pp. 701-705.

[10] R. A. DeCarlo, S. H. Zak, G. P. Mathews "Variable structure control of nonlinear multivariable system: A tutorial," Proceedings of the IEEE, Vol. 76, No.3, March 1988.

[11] Y. Dong and X. Shifan, "Sliding mode control of singular perturbation systems," IEEE International Conference on Systems, Man, and Cybernetics, Vol. 1, Oct.1996, pp. $113-116$.

[12] J. De Leon-Morales, J. G. Alvarez-Leal, R. Castro-Linares, "Speed and position control of flexible joint robot manipulator via a nonlinear control-observer scheme," Proceedings of the IEEE International Conference on Control Applications, October 1997, pp.312-317.

[13] J. C. Doyle, K. Glover, P. P. Khargonekar, B. A. Francis, " State space solution to standard $\mathrm{H}_{2}$ and $\mathrm{H}_{\infty}$ control problems," IEEE Transactions on Automatic Control, Vol. 34, No. 8, August 1989, pp. 831- 847.

[14] S. V. Drakunov, V. I. Utkin, "Sliding mode in dynamic systems," International Journal of Control, Vol. 55, No. 4, 1992, pp 1029-1037.

[15] O. M. E. EL-Ghezawi, A. S. I. Zinober, S.A. Billings, "Analysis and design of variable structure system using a geometric approach," International Journal of Control, Vol.38, No. 3,1983, pp 657-671.

[16] A. Elshabrawy, H. M. Schwartz, "Fuzzy sliding mode control for a singularly perturbed system," NAFIPS-IEEE conference, June 2004, 
[17] A. Elshabrawy, H. M. Schwartz, V. C. Aitken, " Sliding mode control for singularly perturbed system," IEEE Asian Control Conference, July 2004.

[18] W. Feng, "Characterization and computation for the bound $\varepsilon^{*}$ in linear time-invariant singularly perturbed systems," Systems and Control Letters, Vol. 11, 1988, pp 195 202.

[19] W. Gao, J. C. Hung, "Variable structure control of nonlinear systems: A new approach," IEEE Transactions on Industrial Electronics, Vol. 40, No. 1, Feb. 1993.

[20] J. A. Galleos, " Sliding mode control of induction motors using singular perturbation techniques," First IEEE Conference on Control Applications, Vol. 2, 1992, pp. 922 927.

[21] N. K. Gupta, "Frequency shaped cost functionals: extension of linear quadratic gaussian design method, " Journal of Guidance and Control, Vol. 3, Nov.-Dec 1980, pp. 529-535.

[22] B. S. Heck, "Sliding mode control for singularly perturbed systems," International Journal of Control, Vol. 53, No. 4, 1991, pp. 985-1001.

[23] J. Y. Hung, W. Gao, J. C. Hung, "Variable structure control: A survey," IEEE Transactions on Industrial Electronics, Vol. 40, No. 1, Feb 1993, pp. 2- 22.

[24] L. R. Hunt, R. Su, G. Meyer, "Global transformation of nonlinear systems," IEEE Transactions on Automatic Control, Vol. AC-31, No11, 1986, pp. 24- 31

[25] M. Innocenti, L. Greco, L. Pollini, "Stability issues in dual time scale systems," Proceedings of the American Control Conference, 2002, pp. 3914-3921.

[26] M. Innocenti, L. Greco, L. Pollini, " Sliding mode control for two-time scale systems: stability issue," Automatica, Vol. 39, September 2003, pp. 273- 280. 
[27] A. Ishigame, T. Furukawa, S. Kawamoto "Sliding mode controller design based on fuzzy inference for nonlinear systems," IEEE Transactions on Industrial Electronics, Vol. 40 , No. 1, Feb. 1993 , pp. $64-70$

[28] I. Kalaykov, B. Miev, "Time optimal sliding mode control of robot manipulator," IEEE 26 Annual Conference of the Industrial Electronic Society, Oct 2000, pp. 265 270.

[29] H. K. Khalil, "Nonlinear systems," NJ: Prentice Hall, 2002.

[30] P. Kokotovic, H. K. Khalil, J. O'Reilly, "Singular perturbation methods in control, Analysis and design," Society for Industrial and Applied Mathematics, Academic Press, 1999.

[31] P. Kokotovic, A. Bensoussan, G. Blankenship "Singular perturbations and asymptotic analysis in control systems," New York: Springer-Verlag, 1987.

[32] A. J. Koshkouei, A. S. Zinober, "Robust frequency shaping sliding mode control, " IEE Proceedings of Control Theory Applications, Vol. 147, No. 3, May 2000, pp. 312-320.

[33] C.C. Lee, "Fuzzy logic in control system: fuzzy logic controller part I," IEEE Transactions on Systems Man and Cybernetics, Vol. 20, No. 2, March/April 1990, pp. $404-418$

[34] C.C. Lee, "Fuzzy logic in control system: fuzzy logic controller part II," IEEE Transactions on Systems Man and Cybernetics, Vol. 20, No. 3, March/April 1990, pp. 419- 435 . 
[35] H. Lee, E. Kim and H. Kang, M. Park, " Design of a sliding mode controller with fuzzy sliding surfaces," IEEE Proceedings on Control Theory and Applications, Vol. 145, No. 5, September 1998, pp. 411-418.

[36] H-. R. Lin, W-. J. Wang, "Fuzzy control design for the prespecified trajectory tracking with sliding mode," IEEE International Conference on Computational Intelligence, Vol. 1, 1998, pp 292-295.

[37] T. S. Li, "Composite sliding mode control of singularly perturbation systems," Proceedings of the American Control Conference, June 1995, pp. 2248-2249.

[38] J. C. Lo, Y. H. Kuo, "Decoupled fuzzy sliding mode control," IEEE Transactions on Fuzzy System, Vol. 6, No. 3, August 1998, pp. 426- 435

[39] A. G. Luk'yanov, V. I. Aitken, "Method of reduced equation for dynamic system to a regular form," Automatic Remote Control, Vol. 42, No. 4, 1981, pp413-422.

[40] D. S. Naidu, "Singular perturbation methodology in control systems," IEE series, United Kingdom: Peter Peregrinus, 1988.

[41] N. K. Nishimura, H. Tian, " $H_{\infty} / \mu$ control based frequency shaped sliding mode control for flexible structure," JSME international journal, series C, 1996, pp. 493504.

[42] R. Palm and D. Driankov, "Model based fuzzy control," Berlin, Springer, 1997.

[43] Shao, "Fuzzy self organizing controller and its application for dynamic processes," Fuzzy Sets and Systems, Vol. 26, 1988, pp151-164.

[44] H. Sira-Ramirez, "Differential geometric methods in variable structure control," International Journal of Control, Vol. 48, No. 4, 1988, pp. 1359-1390. 
[45] H. Sira-Ramirez, "Sliding regimes in general non-linear systems: a relative degree approach, " International Journal of Control, Vol.50, No. 4, 1989, pp.1487-1506.

[46] R. S. Sanchez-Pena, Mario Sznaier "Robust system theory and application," John Wiley, 1998.

[47] J. J. Slotine, W. Li, "Applied nonlinear control," Englewood cliffs, N. J, Prentice Hall, 1991.

[48] J. J. Slotine, "Sliding controller design for non-linear system," International Journal of Control, Vol. 40, No. 2, 1984, pp. 421-434.

[49] J. J. Slotine, S. S. Sastry, "Tracking control of non-linear systems using sliding surfaces with application to robot manipulators," International Journal of Control, Vol.38, 1983, pp. $465-492$.

[50] J. J. Slotine and S. Hong, "Two time sliding control of manipulators with flexible joints," Proceedings of the American Control Conference, 1986, pp. 805-810.

[51] D. R. Smith, "Singular-perturbation theory: an introduction with applications," New York : Cambridge University Press, 1985.

[52] R. H. C. Takahashi, P. L. D. Peres, " $H_{\infty}$ design of switching surfaces for sliding mode control with non-matching disturbances," Proceedings of the $36^{\text {th }}$ Conference on Decision and Control, California, Dec. 1997, pp. 4046-4051.

[53] T. Takagi, M. Sugeno, "Fuzzy identification of systems and its application to modeling and control," IEEE Transactions on Systems, Man and Cybernetics, Vol. SMC-15, No. 1, 1985, pp.116-132.

[54] V. I. Utkin, "Sliding mode in control optimization," Springer- Verlag, 1992. 
[55] V. I. Utkin, "Sliding mode control design principles and applications to electric drives," IEEE Transactions on Industrial Electronics, Vol. 40, No. 1, Feb. 1993, pp. 23- 36 .

[56] V. I. Utkin, "Variable structure systems with sliding mode", IEEE Transactions on Automatic Control, Vol. AC22, No. 2, April 1977, pp. 212-222.

[57] V. I. Utkin, K. D. Young, " Methods for constructing discontinous planes in multidimensional variable structure systems," Automation and Remote control, No. 31,1978, pp.1466-1470.

[58] Wang, A. B. Rad, P. T. Chan, "Indirect adaptive fuzzy sliding mode control: Part I: fuzzy switching," Fuzzy Sets and Systems, Vol. 122, 2001, pp.21-30.

[59] W. Wang and H. Lin, " Fuzzy control design for trajectory tracking on uncertain nonlinear systems," IEEE Transactions on Fuzzy Systems, Vol. 7, No. 1, February 1999, pp. 53-62.

[60] Wu-Chang $\mathrm{Su}$, "Sliding surface design for singularly perturbed systems," Proceedings of the American Control Conference, June 1998, pp. 1508-1512.

[61] K. D. Young, U. Ozguner, "Frequency shaping compensator design for sliding mode," International Journal of Control, Vol. 57, No. 5, 1993, pp. 1005-1019.

[62] K. D. Young, U. Ozguner, "Sliding mode: control engineering in practice," Proceeding of the American Control Conference, Jun. 1999, pp. 150-162.

[63] K. Zhou, J. C. Doyle, " Essentials of robust control," Printece Hall, Second Addition, 2002.

[64] L. A. Zadah, "Fuzzy sets," Information and Control, Vol. 8, 1965, pp 338-353. 
[65] Q. P. Ha, D. C. Rye and H. F. Durrant-Whyte, " Fuzzy moving sliding mode control with applications to robotic manipulators," Automatica, Vol. 35, 1999, pp.607-616. 


\section{Appendix A}

This appendix provides the proof for theorem 3.1 in chapter 5

Consider a nonlinear singularly perturbed system [30]

$$
\begin{aligned}
& \dot{x}=f(x, z) \\
& \varepsilon \dot{\varepsilon}=g(x, z)
\end{aligned}
$$

where $x \in R^{n}$ and $z \in R^{m}$. It is assumed that equation (A.1) and equation (A.2) have a unique solution for given initial conditions. Assume also that the system given by equations (A.1) and (A.2) have equilibrium points at the origin. The stability of the origin can be investigated using the reduced slow system

$$
\dot{x}=f\left(x, z_{s}\right)=f_{r}(x)
$$

where $z_{s}=h(x)$ is an isolated root of $0=g(x, z)$ and represents the steady state solution of the fast states. In the quasi steady state method, we assume that the slow variable are constant during the fast transients, that is $\dot{z}=0$ and $x=x_{s}=$ constant. Then the fast variations are the deviation of $z$ from its quasi steady state. Fast states can be given by,

$x_{f}=z-z_{s}=z-h(x)$

$x_{f}=g\left(x,\left(z-z_{s}\right)\right)$

We start by stating the assumptions.

\section{Assumption 1}

The reduced system given by equation(A.3) has a Lyapunov function $V: R^{n} \rightarrow R_{-}$ such that for all $x \in R^{n}$ 


$$
\frac{\partial V}{\partial x} f_{r}(x) \leq-\alpha_{1} \psi^{2}(x), \quad \alpha_{1}>0
$$

where $\psi(x)$ is a scalar valued function of $x$ that vanishes at $x=0$ and is different from zero for all other $x \in R^{n}$

\section{Assumption 2}

The boundary layer system given by equation (A.2) has a Lyapunov function $W(x, z): R^{n} \times R^{m} \rightarrow R_{+}$such that for all $x \in R^{n}$ and $z \in R^{m}$

$$
\frac{\partial W}{\partial z} g(x, z) \leq-\alpha_{2} \phi^{2}\left(z-z_{s}\right) \quad \alpha_{2}>0
$$

where $\phi\left(z-z_{s}\right)$ is a scalar valued function of $\left(z-z_{s}\right) \in R^{m}$ that vanishes at $z=z_{s}$ and is different from zero for all other $x \in R^{n}$ and $z \in R^{m}$

\section{Assumption 3}

The following two inequalities hold $\forall x \in R^{n}$ and $z \in R^{m}$
a) $\frac{\partial W}{\partial x} f(x, z) \leq \gamma \phi^{2}\left(z-z_{s}\right)+\beta_{2} \psi(x) \phi\left(z-z_{s}\right)$
b) $\frac{\partial V}{\partial x}\left(f(x, z)-f\left(x, z_{s}\right) \leq \beta_{I} \psi(x) \phi\left(z-z_{s}\right)\right.$

The constants $\beta_{1}, \beta_{2}$ and $\gamma$ are nonnegative. Condition 1) guarantee that $x=0$ is an asymptotically stable equilibrium point of the reduced system (A.3). Condition 2) plays the same role for the system given by equation (A.4), which has an equilibrium point $z=z_{s}$; that is why in condition 2) the comparison function $\phi($.$) depends on z-z_{s}$. It is important to notice that condition 2 should hold uniformly in $x$, i.e. the positive constant $\alpha_{2}$ should be independent of $x$. Inequalities (A.7) and (A.8) determine the permissible interaction between the slow and fast variable. They are basically smoothness 
requirements of $f$ and $g$. One way to grasp their meaning is to consider a special case when the partial derivative of $V$ and $W$ are bounded by $\psi$ and $\phi$, respectively, and $f_{r}(x)$ is bounded by $\psi$.

Inequalities (A.7) and (A.8) simply says that the rate of growth of $f$ in $z$ cannot be faster than the rate of growth of $\phi($.$) . Condition (A.7) will drop out if W$ is not a function of $x$. However, choosing $W$ independent of $x$ may be impossible or if possible, would make condition (A.6) very restrictive. Finally, inequality (A.7) determines the permissible dependence of $g$ on $\varepsilon$. It will drop out if $g$ is independent of $\varepsilon$. It is believed that conditions 1) -3 ) are mild.

\section{Theorem}

Suppose that assumptions 1) -3) hold; let $d$ be a positive number such that $0<d<1$, and let $\varepsilon^{*}(d)$ be the positive number given by

$$
\varepsilon^{*}(d)=\frac{\alpha_{1} \alpha_{2}}{\alpha_{1} \gamma+\left[\beta_{1}(1-d)+\beta_{2} d\right]^{2} / 4 d(1-d)}
$$

Then for all $\varepsilon<\varepsilon^{*}$ the origin $(x=0, z=0)$ is an asymptotic stable equilibrium point of (A.1)\& (A.2) and

$v(x, z)=(1-d) V(x)+d W(x, z)$

Is Lyapunov function for equation (A.1)\& (A.2).

\section{Proof:}

Let $v(x, z)$ denote the derivative of $v$ along the trajectory of system given by equations (A.1) and (A.2). We have 


$$
\begin{aligned}
\dot{v}(x, z)= & (1-d) \frac{\partial V}{\partial x} f(x, z)+\frac{d}{\varepsilon} \frac{\partial W}{\partial z} g(x, z)+d \frac{\partial W}{\partial x} f(x, z) \\
\dot{v}(x, z)= & (1-d) \frac{\partial V}{\partial x} f_{r}(x)+(1-d) \frac{\partial V}{\partial x}\left[f(x, z)-f\left(x, z_{s}\right)\right] \\
& +\frac{d}{\varepsilon} \frac{\partial W}{\partial z} g\left(x, z_{s}\right)+d \frac{\partial W}{\partial x} f(x, z) \\
\dot{v}(x, z) \leq & -\alpha_{1}(1-d) \psi^{2}(x)+(1-d) \beta_{l} \psi(x) \phi\left(z-z_{s}\right) \\
& -d\left[\frac{\alpha_{2}}{\varepsilon}-\gamma\right] \phi^{2}\left(z-z_{s}\right)+d \beta_{2} \psi(x) \phi\left(z-z_{s}\right)
\end{aligned}
$$

Thus

$$
\begin{aligned}
\dot{v}(x, z) \leq & -\left[\begin{array}{c}
\psi(x) \\
\phi\left(z-z_{s}\right)
\end{array}\right]^{T} \times \\
& {\left[\begin{array}{cc}
(1-d) \alpha_{1} & -1 / 2(1-d) \beta_{1}-1 / 2 d \beta_{2} \\
-1 / 2(1-d) \beta_{I}-1 / 2 d \beta_{2} & d\left(\frac{\alpha_{2}}{\varepsilon}-\gamma\right)
\end{array}\right]\left[\begin{array}{c}
\psi(x) \\
\phi\left(z-z_{s}\right)
\end{array}\right] } \\
\dot{v}(x, z) \leq- & {\left[\begin{array}{cc}
\psi(x) \\
\phi\left(z-z_{s}\right)
\end{array}\right]^{T} T\left[\begin{array}{c}
\psi(x) \\
\phi\left(z-z_{s}\right)
\end{array}\right] } \\
\text { where } T= & {\left[\begin{array}{cc}
(1-d) \alpha_{1} & -1 / 2(1-d) \beta_{1}-1 / 2 d \beta_{2} \\
-1 / 2(1-d) \beta_{1}-1 / 2 d \beta_{2} & d\left(\frac{\alpha_{2}}{\varepsilon}-\gamma\right)
\end{array}\right] }
\end{aligned}
$$

For asymptotic stability of system given by equations (A.1) and (A.2), it is sufficient to require that $T$ be a positive definite matrix. For any choice of $d(0<d<l), T$ will be positive definite when $\varepsilon$ is sufficiently small. In particular, $T$ is positive definite for all $\varepsilon<\varepsilon^{*}(d)$

Remark 1: if $V(x)$ and $W(x, z)$ are radially unbounded the origin $(x=0, y=0)$ will be asymptotically stable in the large. 
An interesting point in the theorem is the arbitrariness of $d$. By allowing $d$ to take any value on the interval $(0,1)$, the composite Lyapunov function $v(x, z)$ as given by equation (A.10), covers all possible linear combination of $V$ and $W$. According to the theorem, any one of these linear combinations is a Lyapunov function for the singularly perturbed system as $\varepsilon \rightarrow 0$. What is more interesting is that for a given $d$, theorem 1 ) provides us with the upper bound $\varepsilon^{*}$. Figure 1 shows a sketch of $\varepsilon^{*}(d)$ versus $d$. From that sketch we see that $\varepsilon^{*}(d)$ has a maximum value $\varepsilon^{*}$ at $d=d_{*}$. Straightforward calculations show that

$$
\varepsilon^{*}=\frac{\alpha_{1} \alpha_{2}}{\alpha_{1} \gamma+\beta_{1} \beta_{2}}
$$

and

$$
d_{*}=\frac{\beta_{1}}{\beta_{1}+\beta_{2}} .
$$

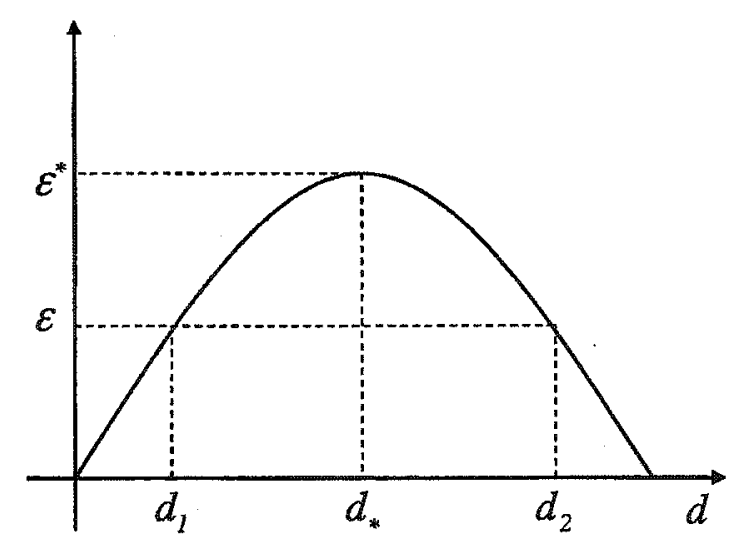

Figure 1: stability upper bound of $\varepsilon$ 